Florida International University FIU Digital Commons

\title{
Screening of Plants for Antibacterial Properties: Growth Inhibition of Staphylococcus aureus by Artemisia Tridentata
}

Steven Ross Eichelbaum

Florida International University, eichelbs@fiu.edu

DOI: $10.25148 /$ etd.FIDC004040

Follow this and additional works at: https:// digitalcommons.fiu.edu/etd

Part of the Biology Commons, Ecology and Evolutionary Biology Commons, Microbiology Commons, Molecular Biology Commons, and the Plant Sciences Commons

\section{Recommended Citation}

Eichelbaum, Steven Ross, "Screening of Plants for Antibacterial Properties: Growth Inhibition of Staphylococcus aureus by Artemisia Tridentata" (2016). FIU Electronic Theses and Dissertations. 3526.

https://digitalcommons.fiu.edu/etd/3526 


\section{FLORIDA INTERNATIONAL UNIVERSITY}

Miami, Florida

\section{SCREENING OF PLANTS FOR ANTIBACTERIAL PROPERTIES: GROWTH}

INHIBITION OF STAPHYLOCOCCUS AUREUS BY ARTEMISIA TRIDENTATA

A dissertation submitted in partial fulfillment of

the requirements for the degree of

DOCTOR OF PHILOSOPHY

in

BIOCHEMISTRY

by

Steven Eichelbaum 
To: Dean Michael R. Heithaus

College of Arts, Sciences and Education

This dissertation, written by Steven Eichelbaum, and entitled Screening of Plants for Antibacterial Properties: Growth Inhibition of Staphylococcus aureus by Artemisia tridentata, having been approved in respect to style and intellectual content, is referred to you for judgment.

We have read this dissertation and recommend that it be approved.

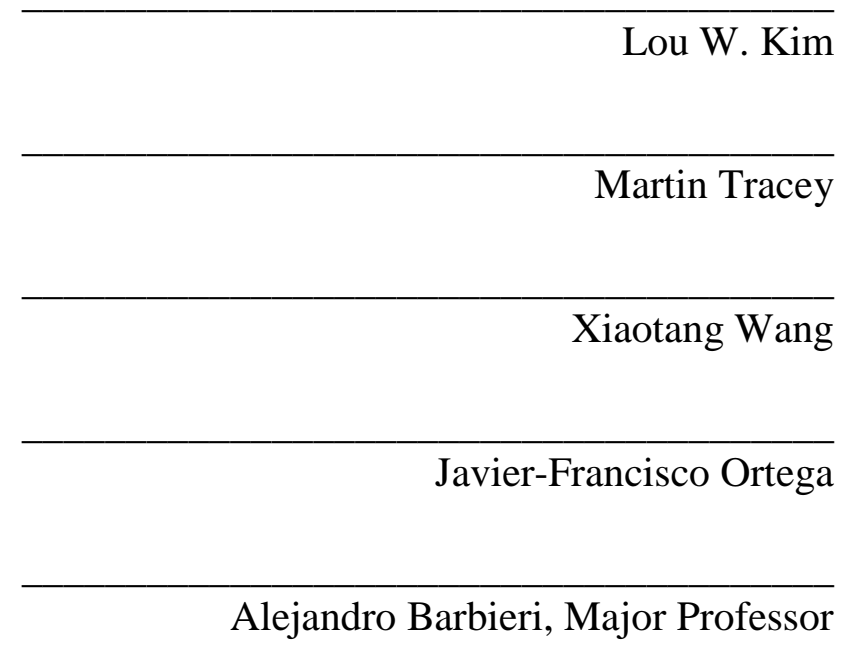

Date of Defense: November 4, 2016

The dissertation of Steven Eichelbaum is approved.

Dean Michael R. Heithaus College of Arts, Sciences and Education

Andrés G. Gil

Vice President for Research and Economic Development and Dean of the University Graduate School

Florida International University, 2017 


\section{ACKNOWLEDGMENTS}

I would first like to acknowledge Dr. Alejandro Barbieri for allowing me into his lab, and for providing me with the guidance and materials necessary to complete my project. I would also like to acknowledge Dr. Kathleen Rein for providing guidance in aspects of the project related to organic chemistry. Further, I would acknowledge Chemistry Department Graduate Secretary Maggie Autie for her overall helpfulness and willingness to listen to my constant grievances. Lastly, I would acknowledge the International Center for Tropical Botany for providing the Fellowship funding which allowed me to complete my studies. 
ABSTRACT OF THE DISSERTATION

SCREENING OF PLANTS FOR ANTIBACTERIAL PROPERTIES: GROWTH

INHIBITION OF STAPHYLOCOCCUS AUREUS BY ARTEMISIA TRIDENTATA

by

Steven Eichelbaum

Florida International University, 2017

Miami, Florida

Professor Alejandro Barbieri, Major Professor

Drug-resistant pathogenic and opportunistic bacteria are increasing in occurrence and prevalence, and pose a dangerous threat to human health. In the search for novel antibiotics with which to combat this threat, plants, specifically those used in traditional medicine with ascribed antibacterial properties, offer a promising and potentially vast source of such therapeutic compounds. The purpose of this study was therefore to screen chemical extracts created from various plant species for antibacterial properties versus pathogenic bacterial species. In the course of these antibacterial assays, we successfully identified a methanol extract derived from Artemisia tridentata tridentata plant material as capable of inhibiting the growth of the opportunistic pathogen Staphylococcus aureus. Three sub-fractions were created using hexane, ethyl acetate and water solvents. Each of these extracts displayed significant antibacterial activity versus a wild-type strain over a period of six hours, at concentrations as low as $62.5 \mu \mathrm{g} / \mathrm{ml}$. The extracts also demonstrated an enhancement of antibiotic effects when combined with ampicillin, G418 sulfate or amikacin, for a period of up to twelve hours. Though the efficacy of the extracts was lessened when tested against an ampicillin-resistant strain, significant 
enhancement of the efficacy of this antibiotic was still observed. Gas chromatographymass spectrometry analysis of these three extracts revealed the sesquiterpene lactone achillin as present in each. Column chromatography of the hexane extract resulted in a fraction retaining its antibacterial activity, and still containing this compound, further implicating it as responsible for the antibacterial activity of this plant. The results of serial dilution and plating of extract-treated samples, along with those of ethidium bromide assays and transmission electron microscopy analysis, indicated a bacteriostatic mechanism of action involving disruption of the bacterial membrane, which is in agreement with the literature on the antibacterial properties of this plant, and those of sesquiterpene lactones, respectively. We therefore conclude that achillin, likely produced as a secondary metabolite by Artemisia tridentata tridentata, possesses growth inhibitory properties versus Staphylococcus aureus, and should be isolated and studied further for the purposes of evaluating its potential use, either as a stand-alone antibiotic, or as an adjunctive therapeutic, in the treatment of drug-resistant bacterial pathogens. 


\section{TABLE OF CONTENTS}

CHAPTER

PAGE

I. INTRODUCTION

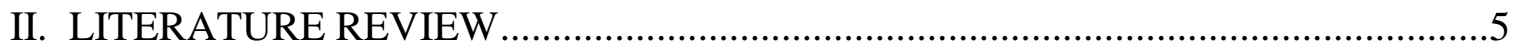

Why Plants? ............................................................................................ 5

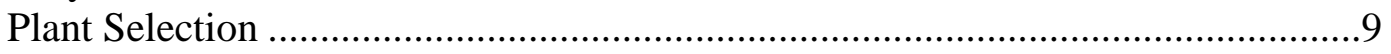

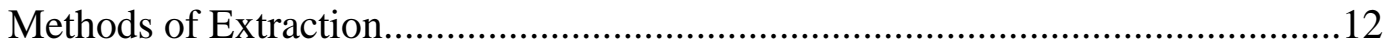

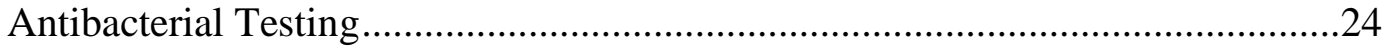

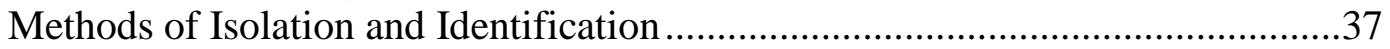

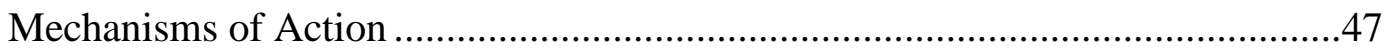

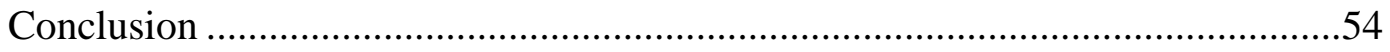

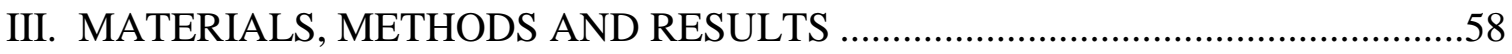

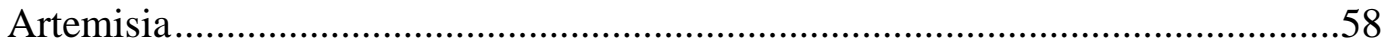

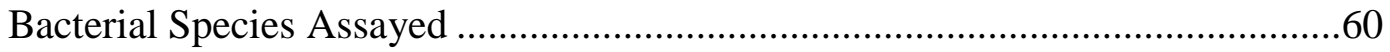

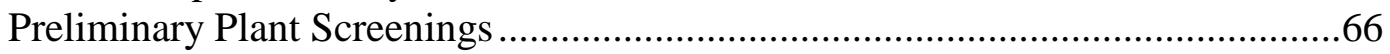

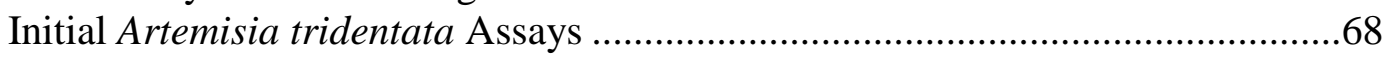

Growth Inhibition and Combination With Antibiotics ........................................ 74

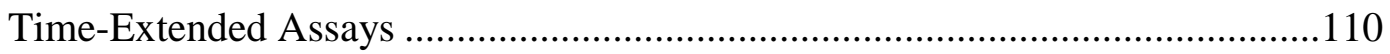

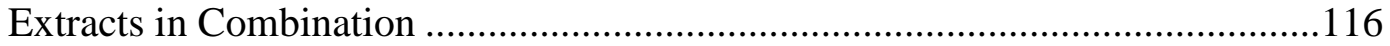

Ampicillin-Resistant Staphylococcus aureus ...............................................120

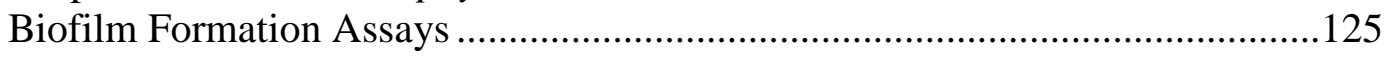

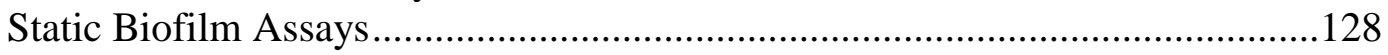

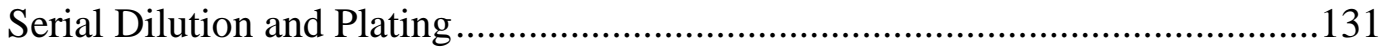

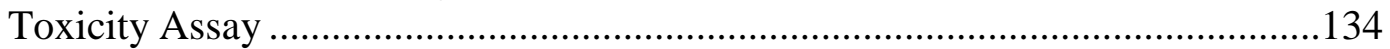

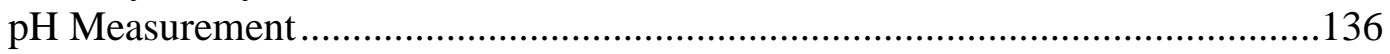

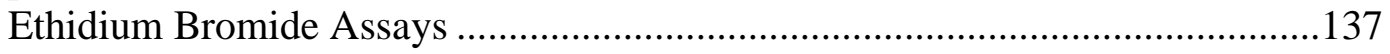

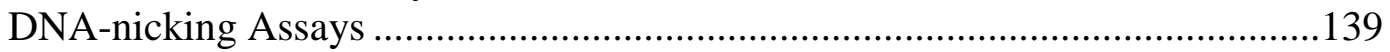

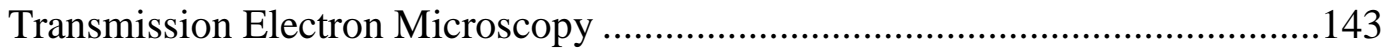

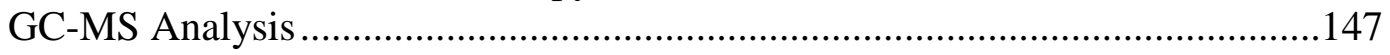

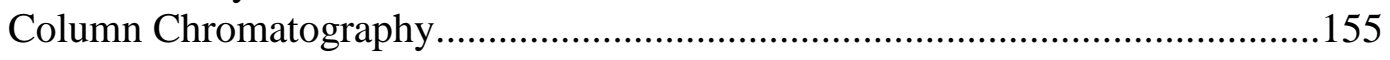

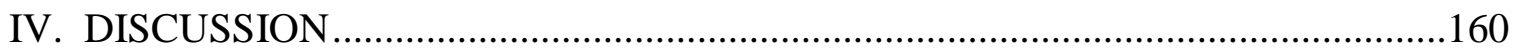

Evaluation of Results ................................................................................ 160

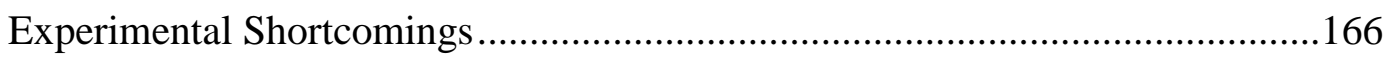

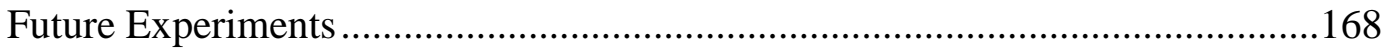

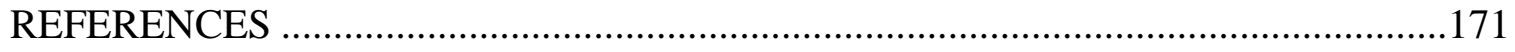

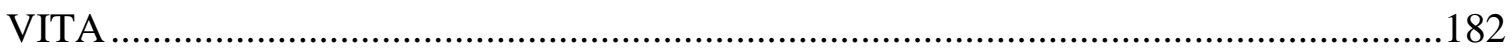




\section{LIST OF TABLES}

TABLE

PAGE

1. Summary of preliminary plant screening assays .................................................66

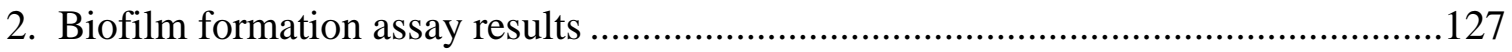

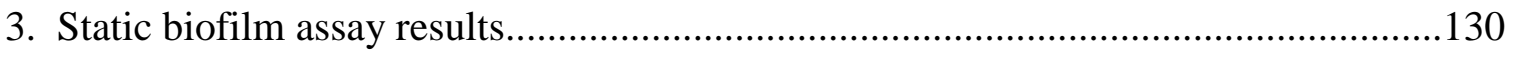

4. Serial and dilution of $A$. tridentata extract-treated $S$. aureus .................................133

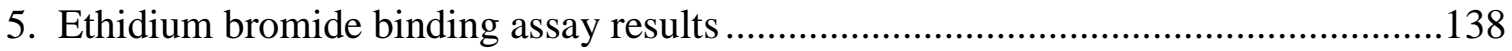

6. Summary of GC-MS results from hexane extract analysis....................................151

7. Summary of GC-MS results from ethyl acetate extract analysis ............................152

8. Summary of GC-MS results from water extract analysis ....................................154

9. Summary of GC-MS results from $10 \%$ acetonitrile column fraction analysis ..........159 


\section{LIST OF FIGURES}

FIGURE

PAGE

1. S. aureus treated with A. tridentata methanol extract .............................................69

2. P. aeruginosa treated with $A$. tridentata methanol extract ....................................69

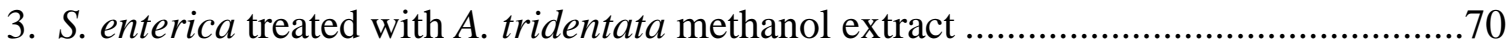

4. L. monocytogenes treated with A. tridentata methanol extract.................................70

5. $2^{\text {nd }}$ trial of $S$. aureus treated with A. tridentata methanol extract ............................... 71

6. $3^{\text {rd }}$ trial of $S$. aureus treated with A. tridentata methanol extract...............................73

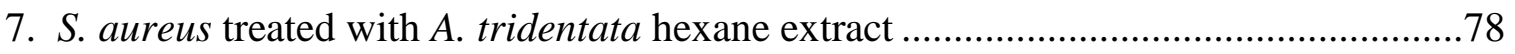

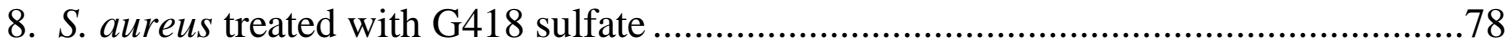

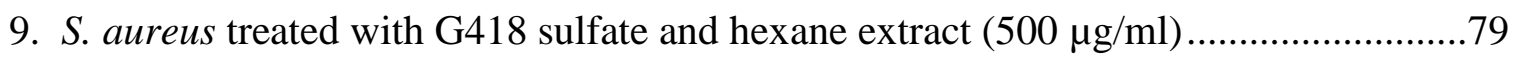

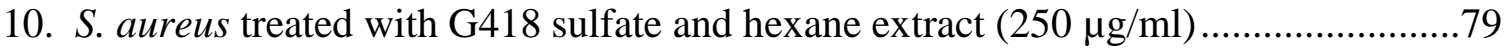

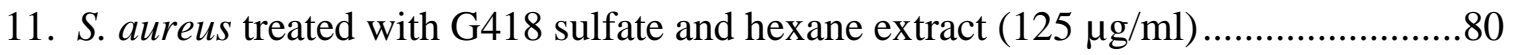

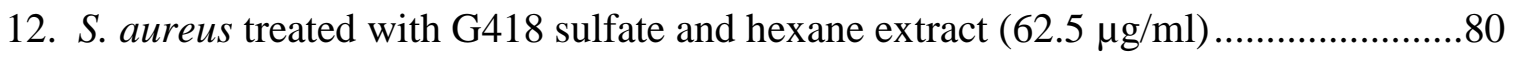

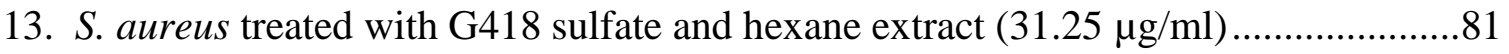

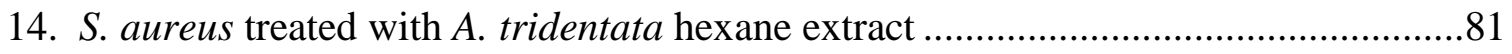

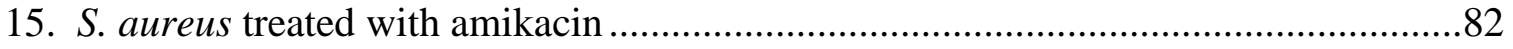

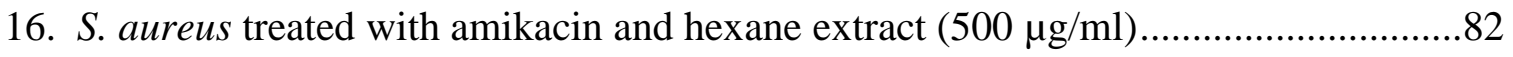

17. S. aureus treated with amikacin and hexane extract $(250 \mu \mathrm{g} / \mathrm{ml}) \ldots \ldots \ldots \ldots \ldots \ldots \ldots \ldots \ldots . . . . . . \ldots 3$

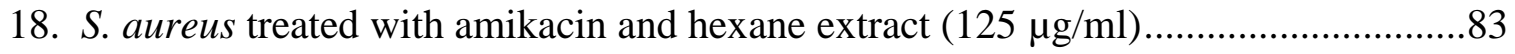

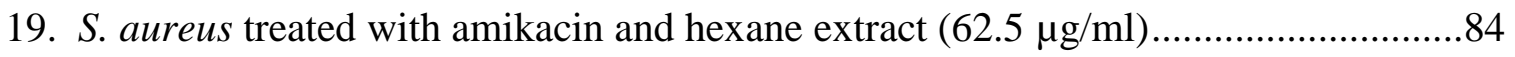

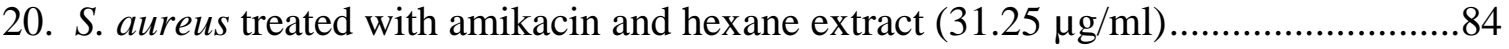


21. S. aureus treated with $A$. tridentata hexane extract

22. S. aureus treated with ampicillin .85

23. S. aureus treated with ampicillin and hexane extract $(500 \mu \mathrm{g} / \mathrm{ml})$..... .86

24. S. aureus treated with ampicillin and hexane extract $(250 \mu \mathrm{g} / \mathrm{ml})$ .86

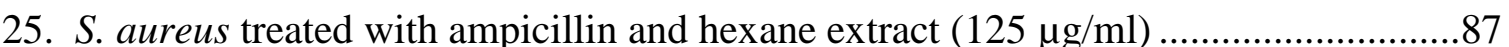

26. S. aureus treated with ampicillin and hexane extract $(62.5 \mu \mathrm{g} / \mathrm{ml})$ .87

27. S. aureus treated with ampicillin and hexane extract $(31.25 \mu \mathrm{g} / \mathrm{ml})$ .88

28. S. aureus treated with $A$. tridentata ethyl acetate extract .88

29. S. aureus treated with G418 sulfate .89

30. S. aureus treated with G418 sulfate and ethyl acetate extract $(500 \mu \mathrm{g} / \mathrm{ml})$ .89

31. S. aureus treated with G418 sulfate and ethyl acetate extract $(250 \mu \mathrm{g} / \mathrm{ml})$ .90

32. S. aureus treated with G418 sulfate and ethyl acetate extract $(125 \mu \mathrm{g} / \mathrm{ml}) \ldots \ldots \ldots \ldots \ldots . . .90$

33. S. aureus treated with G418 sulfate and ethyl acetate extract $(62.5 \mu \mathrm{g} / \mathrm{ml}) \ldots \ldots \ldots \ldots . . .91$

34. S. aureus treated with G418 sulfate and ethyl acetate extract $(31.25 \mu \mathrm{g} / \mathrm{ml})$...... .91

35. S. aureus treated with $A$. tridentata ethyl acetate extract .92

36. S. aureus treated with amikacin .92

37. S. aureus treated with amikacin and ethyl acetate extract $(500 \mu \mathrm{g} / \mathrm{ml})$ 93

38. S. aureus treated with amikacin and ethyl acetate extract $(250 \mu \mathrm{g} / \mathrm{ml})$ .93

39. S. aureus treated with amikacin and ethyl acetate extract $(125 \mu \mathrm{g} / \mathrm{ml})$ .94

40. S. aureus treated with amikacin and ethyl acetate extract $(62.5 \mu \mathrm{g} / \mathrm{ml})$. .94

41. S. aureus treated with amikacin and ethyl acetate extract $(31.25 \mu \mathrm{g} / \mathrm{ml})$. .95

42. S. aureus treated with $A$. tridentata ethyl acetate extract .95

43. S. aureus treated with ampicillin .96 
44. S. aureus treated with ampicillin and ethyl acetate extract $(500 \mu \mathrm{g} / \mathrm{ml}) \ldots \ldots \ldots \ldots \ldots \ldots . . . . .96$

45. S. aureus treated with ampicillin and ethyl acetate extract $(250 \mu \mathrm{g} / \mathrm{ml}) \ldots \ldots \ldots \ldots \ldots \ldots . . . . .97$

46. S. aureus treated with ampicillin and ethyl acetate extract $(125 \mu \mathrm{g} / \mathrm{ml})$.................97

47. S. aureus treated with ampicillin and ethyl acetate extract $(62.5 \mu \mathrm{g} / \mathrm{ml}) \ldots \ldots \ldots \ldots \ldots . . . .98$

48. S. aureus treated with ampicillin and ethyl acetate extract $(31.25 \mu \mathrm{g} / \mathrm{ml}) \ldots \ldots \ldots \ldots \ldots . . . .98$

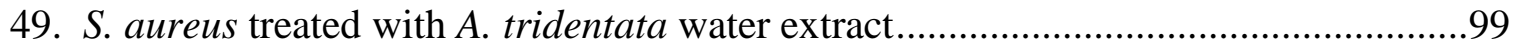

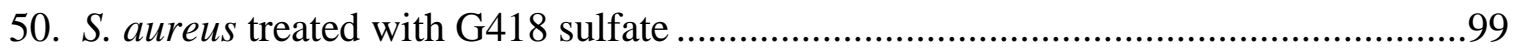

51. S. aureus treated with G418 sulfate and water extract $(500 \mu \mathrm{g} / \mathrm{ml})$.......................100

52. S. aureus treated with G418 sulfate and water extract $(250 \mu \mathrm{g} / \mathrm{ml}) \ldots \ldots \ldots \ldots \ldots \ldots \ldots \ldots . . . . . . . . .100$

53. S. aureus treated with G418 sulfate and water extract $(125 \mu \mathrm{g} / \mathrm{ml})$......................101

54. S. aureus treated with G418 sulfate and water extract $(62.5 \mu \mathrm{g} / \mathrm{ml})$......................101

55. S. aureus treated with G418 sulfate and water extract $(31.25 \mu \mathrm{g} / \mathrm{ml}) \ldots \ldots \ldots \ldots \ldots \ldots \ldots . . . . .102$

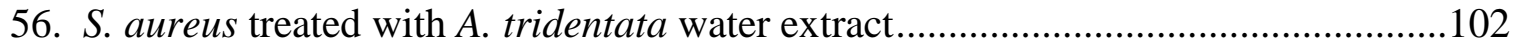

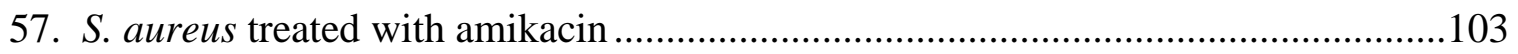

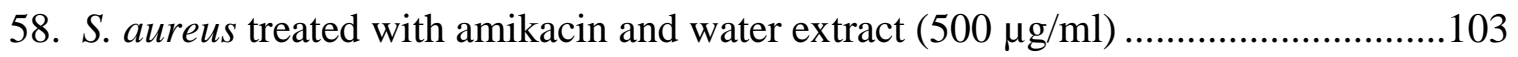

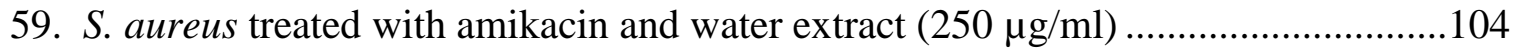

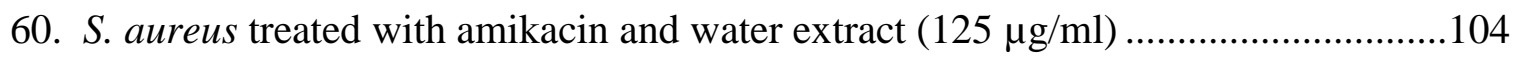

61. S. aureus treated with amikacin and water extract $(62.5 \mu \mathrm{g} / \mathrm{ml})$...........................105

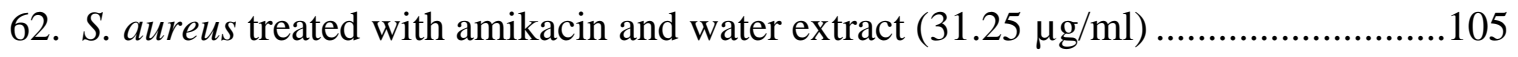

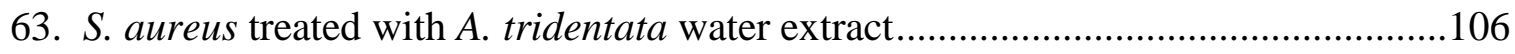

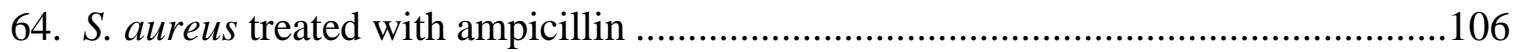

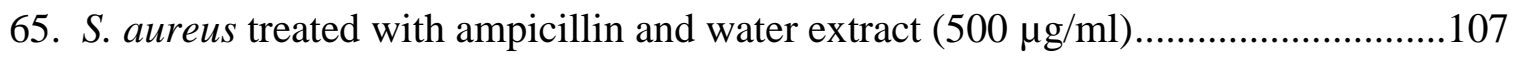

66. S. aureus treated with ampicillin and water extract $(250 \mu \mathrm{g} / \mathrm{ml}) \ldots \ldots \ldots \ldots \ldots \ldots \ldots \ldots \ldots . . . \ldots 107$ 


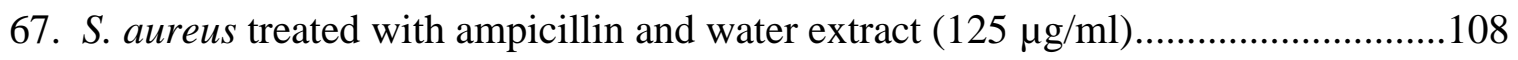

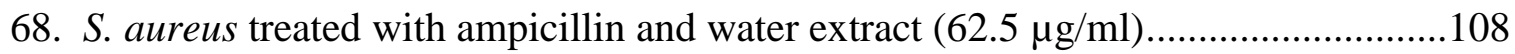

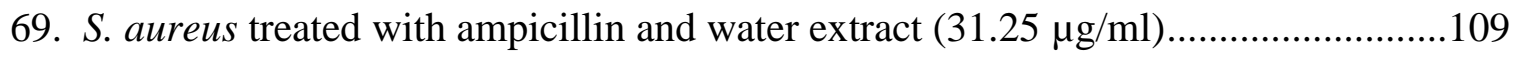

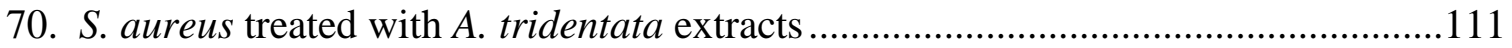

71. S. aureus treated with G418 sulfate, amikacin, ampicillin .................................112

72. S. aureus treated with G418 sulfate and A. tridentata extracts..............................114

73. S. aureus treated with amikacin and A. tridentata extracts....................................114

74. S. aureus treated with ampicillin and A. tridentata extracts .................................115

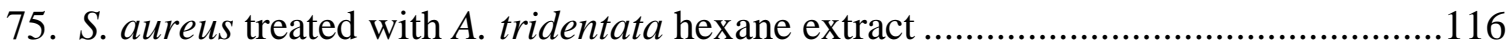

76. S. aureus treated with $A$. tridentata ethyl acetate extract ...................................117

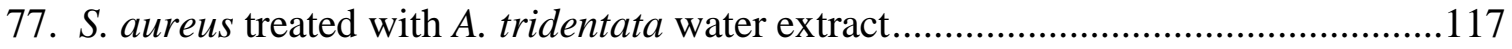

78. S. aureus treated with A. tridentata hexane and ethyl acetate extracts....................118

79. S. aureus treated with $A$. tridentata hexane and water extracts ..............................119

80. S. aureus treated with A. tridentata ethyl acetate and water extracts ......................119

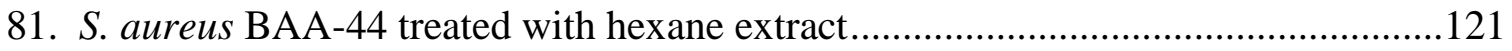

82. S. aureus BAA-44 treated with ethyl acetate extract ..........................................121

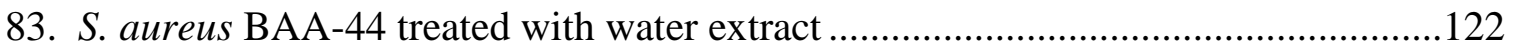

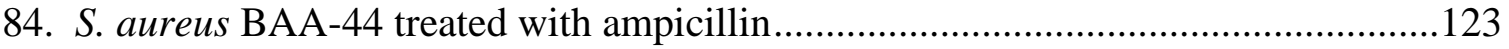

85. S. aureus BAA-44 treated with ampicillin and hexane extract.............................123

86. S. aureus BAA-44 treated with ampicillin and ethyl acetate extract ......................124

87. S. aureus BAA-44 treated with ampicillin and water extract ...............................124

88. Artemisia tridentata ethyl acetate extract-treated MDA-MB-231 cells ...................135

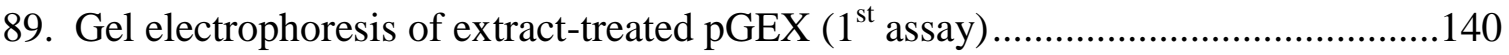


90. Gel electrophoresis of extract-treated pGEX (2nd assay)...................................141

91. Gel electrophoresis of extract-treated pGEX (3rd assay) ....................................142

92. TEM image of untreated Staphylococcus aureus ...............................................145

93. TEM image of water extract-treated Staphylococcus aureus ..................................145

94. TEM image of hexane extract-treated Staphylococcus aureus ...............................146

95. TEM image of ethyl acetate extract-treated Staphylococcus aureus .......................146

96. GC-MS spectral results for Artemisia tridentata hexane extract ............................148

97. GC-MS spectral results for Artemisia tridentata ethyl acetate extract ....................148

98. GC-MS spectral results for Artemisia tridentata water extract ............................149

99. GC-MS results for $10 \%$ acetonitrile column fraction of hexane extract ................157

100. GC-MS results for $100 \%$ acetonitrile column fraction of hexane extract ............157

101. Antibacterial assay of hexane extract column fractions ....................................158 


\section{INTRODUCTION}

Antibiotic-resistant bacteria pose a global health threat. Infectious diseases, increasingly resulting from antibiotic-resistant pathogenic or opportunistic bacteria, represent the leading annual cause of human fatalities ${ }^{1}$. These deaths include a staggering $1 / 2$ of all fatalities in tropical countries, with gastrointestinal infections alone, for example, claiming the lives up to 3 million pre-school aged children per year ${ }^{2}$. In addition, infectious diseases have also arisen as a significant source of morbidity and/or mortality in immunocompromised patients, in both developing and developed countries $^{3,4}$. In HIV cases for example, opportunistic infections represent the leading cause of death in AIDS patients ${ }^{5}$, with bacterial complications both facilitating the infection rate of the HIV virus and reducing the onset time of this disease ${ }^{2}$. Further, there has been an alarming increase in the occurrence of new diseases, and a re-emergence of old ones, accompanied by an increasing prevalence of resistance to antibiotics in clinical use $^{6}$. In fact, despite the availability of more than 200 varieties of antibiotics and chemotherapeutics, the occurrence of multi-drug resistant bacteria is at its peak ${ }^{7}$. The seriousness of the threat posed by antibiotic-resistant bacteria is thus underscored both by its current impact, and the potential ramifications of its increasing prevalence.

The recent reporting of increases in both the number and prevalence of staphylococcal infections, such as those of methicillin-resistant Staphylococcus aureus, combined with the emergence of vancomycin-resistant isolates ${ }^{8}$, serves as evidence of the current impact of antibiotic resistance on human disease. In fact, the level of resistance in clinical isolates of Staphylococcus pneumoniae to antibiotics routinely used for such infections has been reported to be as high as $40 \%$ in some parts of Europe ${ }^{8}$. Therapeutic 
options for methicillin-resistant Staphylococcus aureus (MRSA) have become limited as well, as strains resistant to synthetic antibiotics including macrolides, aminoglycosides, fluroquinolones, chloramphenicol, clindamycin, tetracycline, vancomycin, oxazolidinetype and streptogramin-type antibiotics, in addition to $\beta$-lactams, have emerged ${ }^{9,10}$. It is also worth noting that the increase in the occurrence of drug-resistance has been mirrored by a significant reduction in the number of pharmaceutical companies developing new antimicrobial agents ${ }^{11}$.

However, antibiotic resistant bacteria also have a significant negative impact on agriculture. In fish aquaculture for example, the fast development of the industry, combined with increasing product demands, can lead to overcrowding, poor water quality, or poor nutritional status, which can contribute to stress and immunosuppression in the fish, increasing the risk of disease dissemination ${ }^{12}$. This infection risk necessitates the administration of antimicrobials and other veterinary drugs, which are also used for growth promotion, for prophylactic and therapeutic purposes ${ }^{12}$, all of which are practices which can unfortunately select for antibiotic-resistance. As an example of drugresistance in agricultural animals, chicken and pork have become vehicles for livestockassociated MRSA strains, adding an epidemiological dimension to the pathogen in the food supply ${ }^{13}$.

Alternatively, in crop farming, biocide use at sub-lethal concentrations, in some cases due to limited availability ${ }^{14}$, while in others possibly the result of legal impositions on the application of synthetic antibiotics to crops, hampers disease control, and is another practice which may select for antibiotic resistance ${ }^{15}$. In Europe for example, the protocol of EUREPGAP (European Good Agricultural Practice) places restrictions on the 
allowable residue limits of pesticides on fruits and vegetables ${ }^{16}$, and the use of antibiotic and copper compounds is restricted in many countries over concerns pertaining to human and animal health or the environment ${ }^{14}$. Arguably as a direct result of such policies, several resistant populations of plant pathogens have been reported ${ }^{14}$, and plant diseases caused by plant pathogens, including bacteria, represent a major cause of crop loss ${ }^{17}$. It is therefore worth considering that the threat to human health posed by drug-resistant bacteria in agriculture may be just as great, or even greater than that posed by infectious human diseases, as in agriculture drug resistance threatens both the quantity and safety of the food supply, with food-borne infections currently among the most serious and costly global health concerns ${ }^{18}$.

Two obvious strategies present themselves when considering means to combat the increasing emergence of drug-resistant bacteria. The first would be to remove or reduce the causative factors of bacterial drug-resistance by implementing policies promoting responsible and efficient use of antibiotics, thus reducing "selective pressure" as much as is feasible. The second of course, is the development or discovery of novel antibiotics with unique mechanisms of action which may yet be effective against bacteria otherwise resistant to existing therapeutics. To this end, there has been a renewed interest in exploring "medicinal plants," or plants used in traditional medicine, as possible sources of such pre-existing novel antibiotic compounds. As research of plants for antibiotic compounds is, in recent years if not historically, a relatively underexplored field of study, there is consequentially a lack of standardization in the experimental methods and techniques used in such research. Unfortunately, this discord may limit the ability of an investigator to accurately assess the antibacterial potential of a plant, while also making 
the recognition of significant results, and the comparison of results from separate investigations, difficult. The following therefore begins with an explanation of the reasoning behind the investigation of plants, specifically medicinal plants, as a source of novel antibiotic compounds, then provides a summary and some evaluation of the experimental methods and techniques currently used in this field as they relate to plant selection, chemical extraction, antibiotic screening, the identification of compounds of interest, and the determination of the mechanisms of action of antibacterial compounds. 


\section{LITERATURE REVIEW}

Why Plants?

There are several arguments to be made in support of the research of plants, and medicinal plants in particular, as potential sources of novel antibiotic compounds with which to combat drug resistance. The first of these arguments is to point out the historical role that plants have played in the promotion of human health. A medicinal plant, as defined by the World Health Organization, is a plant which contains substances, in one or more of its parts, which can either be used directly for therapeutic purposes, or are precursors for chemo-pharmaceutical semi-synthesis ${ }^{14}$, and it is important to consider that plants have served as the starting point for many of the modern pharmaceuticals in use today. Indeed, it has been estimated that more than a full quarter of prescribed medications in industrialized nations derive their origins either directly or indirectly from plants $^{19}$. As this historical role relates specifically to plants providing a source of novel antibiotics for the treatment of infectious diseases, it is believed that plants possess secondary metabolites, in various plant tissues, which are produced and used by plants for defensive purposes ${ }^{20,21}$. It has been postulated that these compounds are an evolutionary response to protect the plants from insects, predators, and most importantly in this context, microbes ${ }^{11,22,23}$, and this postulation is directly supported by the discovery that syntheses of some of these compounds occurs post-infection ${ }^{24}$. It is therefore reasonable to hypothesize that some of these compounds produced to fight bacterial plant pathogens may also be beneficial in combating bacterial human pathogens.

Second, plants in general are overwhelmingly abundant, yet vastly underexplored. There exist an estimated 2.5 million species of higher plants, and a large number have yet 
to be studied in this context ${ }^{25,26}$. India alone for example, where much of this type of research in the reviewed literature originates, possesses within its borders approximately 130,000 plant species encompassing some 120 families $^{10}$. In North America, while Native Americans are believed to have used an estimated 2,500 plant species in their traditional medicine practices, the region is believed to contain as many as 20,000 native species $^{27}$. Plants thus represent a potentially vast source of bioactive molecules, which is likely why they have been referred to as the "sleeping giants of the pharmaceutical industry" ${ }^{\prime 19}$. Further, while recognition of the antiseptic qualities of medicinal plants date to antiquity, efforts to characterize these properties in the laboratory setting date only to the early $1900 \mathrm{~s}^{28}$. In fact, the approximately 12,000 secondary metabolites so far isolated from plants are believed to comprise less than $10 \%$ of the total in existence ${ }^{20}$. Therefore, it is entirely plausible that investigation of previously untested plant species will reveal medicinal plants possessing unrecognized antibiotic activities.

The third argument to be made in favor of the research of plants as sources of novel antibiotic compounds is to note that the use of medicinal plants in the practice of traditional medicine persists very prominently even today. The World Health Organization estimates that $80 \%$ of people in developing countries engage in such practices $^{23}$, a number which corresponds to approximately $65 \%$ of the global population. The antimicrobial activity of plant extracts and oils has led to their administration as food preservatives, natural therapies, alternative medicines and pharmaceuticals ${ }^{29}$. While it can be pointed out that there is often little solid scientific evidence to support such claims touting the antibiotic attributes of medicinal plants, it can also be reasonably argued that without thorough scientific evaluation, it would be inappropriate to summarily dismiss 
their virtue. Additionally, given the prevalence of their use, it is necessary to ensure the safety of these plants, as insufficient patient awareness or improper use result in many cases of adverse reactions in traditional medicine, including, among others, allergic reactions, fever and vomiting ${ }^{1}$. Also, in cases where products derived from these plants are indeed found to be beneficial to human health, there is a need for standardization in terms of raw materials, production methods and quality control of finished products ${ }^{1}$. Such safety and standardization issues could be addressed through the increased investigation of these medicinal plants.

Additional arguments to be made in support of the investigation of plants as a potential source of novel antibiotics relate to the potential advantages such products could provide over synthetic antibiotics. There currently exists a public mistrust of synthetic antimicrobials stemming from the potential toxicity or even carcinogenicity of these products $^{30}$, as the use of synthetic antibiotics for example, may in some cases be harmful to distinct organs and threaten consumer health ${ }^{31,32}$. Further, the application of synthetic antimicrobials in agriculture creates additional opportunities for human exposure to such chemicals, either through the consumption of chemical residues on crops, or bioaccumulated chemicals in agricultural animals. Antimicrobials may also be applied to finished food products for preservation and safety purposes, to protect against natural spoilage processes and pathogenic microorganisms, respectively ${ }^{33}$. Consequentially, consumers are increasingly demanding minimally processed foods, at the same time desiring products free from pathogens, yet simultaneously containing fewer synthetic preservatives $^{18,33}$, with mounting pressure from both consumers and legal authorities for alternative, natural product shelf-life extending additives ${ }^{30}$. These demands are becoming 
reflected in public policy. In Europe for example, in-feed antibiotics for livestock were banned by the European Union as far back as $2006^{34}$.

It is noteworthy that plant-derived substances have already served as food preservatives for centuries, with many herbs and spices used in food seasoning in fact also yielding useful medicinal compounds ${ }^{35}$. Additionally, there are some positive results reported for medicinal plants used as animal feed in the reviewed literature. They have been reported to promote growth and appetite, as well as have immunostimulatory and anti-pathogen effects in fish and shrimp aquaculture ${ }^{12}$ for example, and it has been suggested that prophylactic administration of immunostimulants and pro-, pre- and synbiotics is the most promising method of disease control in aquaculture animals ${ }^{36}$. However, it is important to note, as described previously, that there are also existing reports of adverse reactions to traditional medicines. Though the fact that an antibiotic is derived from a natural source may sway public opinion to view it as less hazardous, there are no guarantees to be made as to the advantages, in terms of safety, of substituting plant-derived antibiotics for synthetic antibiotics. Still, it is possible that further plant investigations may identify novel, and comparatively safer antibiotics for clinical and agricultural applications.

Also, from an environmental perspective, the use of plant-derived antibiotics would seem greatly advantageous over the use of synthetic antibiotics. Plant-derived substances, in their natural states, would likely be entirely biodegradable, and thus avoid pollution and environmental degradation issues related to orthodox medicines ${ }^{37}$. Lastly, in comparison to synthetic antibiotics, using plant-derived antibiotics would lessen cost and increase the accessibility to these medications. The possibility, at least in some 
locales, of being able to culture medicinal plants and perhaps even purify or otherwise concentrate substances of medicinal value would be of great benefit to the destitute or those living in geographic areas without reasonable accessibility to modern medications. Thus, given their history as a medicinal source and the number of plant species which have yet to be investigated, their widespread use in traditional medicine and the need to ensure the safety of these practices, as well as the advantages their use may provide over that of synthetic medications, in terms of safety, environmental impact and cost, there is a solid case to be made in favor of the increased and expanded exploration of plants as potential sources of novel antibiotics.

Plant Selection

Studies of plants that investigate their therapeutic potential are typically screening exercises for the evaluation of antioxidant, anti-inflammatory, antifungal or antibacterial properties of plant extracts, and are usually based on ethnobotanical leads ${ }^{38}$. The general strategy of these screening exercises as it pertains specifically to the evaluation of antibacterial properties of the plant can be broken down into several steps; plant selection is naturally the first of these steps. There are both practical and logistical limitations to consider however, before finalizing the selection of a plant or plants for study. In the search for novel antimicrobial compounds, as there are so many species from which to choose, plants with a long standing history of medicinal use constitute the most practical starting point ${ }^{21}$. For example, Tchouya et al. conducted ethnopharmacological surveys in Gabon to identify fifty-two species of medicinal plants used there to treat HIV/AIDSrelated opportunistic diseases, prior to selecting five for phytochemical screening and antibacterial testing ${ }^{5}$. 
However, the availability or accessibility of a medicinal plant must also be taken into consideration before a selection is finalized. If the plant(s) of particular interest require crossing national boundaries to obtain, the likelihood of encountering proprietary ownership issues, as well as the monetary cost, may both increase. Additionally, if it is believed that proprietary issues may arise, estimations should be made prior to the beginning of work, as comprehensively as possible, regarding how much plant sample will be necessary to complete the intended studies. It then needs to be determined if it is possible to procure this amount of plant material, as well as to do so over a reasonable period of time. For example, if a plant(s) is only accessible by means of agreement with a private botanical collection, either foreign or domestic, there may be strict limits imposed on the amount of plant material provided.

Further, if limits are imposed, and on-site cultivation of plants is unfeasible, it should be considered that these limits may unduly prolong studies, potentially creating further issues related to the continuity of the researchers involved, cost, etc., as well as possibly causing variability in experimental results due to chemotype differences in the starting material. These chemotype differences, even among members of the same plant species, may result from geographic ${ }^{39}$ or seasonal growth variations. Smida et al., for example, in investigating the antibacterial activities of Ludwigia peploides and Ludwigia grandiflora over a period of several months, documented time-dependent differences in plant extract efficacy versus multiple strains of bacteria ${ }^{40}$, suggesting temporal differences in plant chemotype. Further, the specific microenvironment in which a plant is grown may also affect its chemotype. According to "plant defense theory," defensive versus growth allocation of plant resources may depend upon the specific light 
environment in which the plant grows, with plants growing in open habitats likely to contain higher levels of defensive compounds and reduced herbivory in comparison to those growing in shaded environments ${ }^{41}$. Thus, as the geographical location, time of sample collection and the microenvironment in which a plant was grown may affect its chemical composition, limited accessibility to a plant may cause difficulty in obtaining plant samples capable of providing consistent experimental results, and this should also be considered prior to the selection of a plant for study.

The selection of which plant part(s) to study is an additional consideration in study design. While it has been stated that antibacterial compounds are more likely to be located within growth buds, young leaves, reproductive organs and parts of annual growth $^{20}$, it is quite evident from the literature that different plant parts possess varying chemotypes, and that antibacterial compounds are not universally relegated to specific tissue types. For example, Yasanuka et al., in testing the antibacterial potential of various Mexican medicinal plants, created extracts from fruits, heartwoods, leaves, fruit peels, roots, stems and twigs, and reported differences in results between plant parts from the same species ${ }^{26}$. The inclusion and separate study of all parts of a selected plant in search of these differences, while appealing, adds substantially to the potential workload and financial cost. Hypothetically, the separate study of individual plant parts is not mandatory, and whole-plant extracts may certainly be used in these screening exercises. However, as will be discussed below, there exists the possibility that if an antibacterial compound(s) present in a plant under study are relegated specifically to one plant part, inclusion of additional plant parts in the creation of an extract may dilute the concentration of this compound(s) to levels low enough to impede detection in 
antibacterial assays. Here again, it would be wise to consult traditional practitioners or users of the medicinal plant(s) of interest, so that plant parts selected for study match those used in practice.

However, the selection of a specific plant part(s) may also increase the logistical difficulty of obtaining samples for study. The harvest of a crop can significantly alter the future growth patterns of a plant population. Mooney et al., for example, found both the number of flowering stalks and the proportion of these flowering stalks to leaves reduced in Ligusticum porteri populations up to two years post-harvest of rhizome and adventitious root material ${ }^{41}$. Generally speaking, the harvesting of plant leaves is more sustainable than that of stems or roots ${ }^{42}$. Therefore, individual suppliers may be understandably reluctant to provide stem or root samples of their plant(s). Thus, the determination of what plant part(s) is of greatest interest, and how this may affect procurement of plant samples, should also be considered when selecting a specific plant(s) for study. In conclusion, while it is in the best interest of the researcher when choosing a plant(s) for study to make practical selections based on ethnobotanical leads, it is also important to take into account how logistical issues pertaining to plant procurement may affect both the availability of plant material and its potential consistency in terms of chemical composition, as well as how the inclusion of various plant parts for study might further complicate this procurement, as well as potentially inflate workload and cost.

Methods of Extraction

Beginning with the treatment of plant material prior to the creation of chemical extracts, there appears in the literature a notable lack of standardization in the 
experimental methods and materials used in this and in subsequent steps of these plant investigations. For example, after sometimes being washed with water, plant material is almost always dried prior to chemical extraction. However, plant material may be dried in the light or in the shade, for varying periods of time, and at different temperatures, and there is no rationale provided by the authors for their method selection. Kuppusamy et al. for example, washed Commelina nudiflora plant material twice in running tap water prior to cutting the material into small pieces, then drying it in the shade at $35^{\circ} \mathrm{C}$ in 12 -hour cycles of light and dark ${ }^{31}$. Alternatively, Voravuthikunchai and Limsuwan, in sampling parts of eight species used in traditional medicine in Thailand, simply cut plant material into small pieces and dried these samples overnight at $60^{\circ} \mathrm{C}^{43}$, whereas Kenny et al. by comparison, sliced and freeze-dried dandelion roots prior to extraction ${ }^{44}$.

Presumably, the purpose of drying plant material is to eliminate excess water and increase the concentrations of any antibacterial compounds present in order to enhance the probability of detection. However, even such a commonplace practice as drying plant material prior to extract creation must be questioned. Alabri et al. for example, reported the comparatively enhanced antimicrobial efficacy of several chemical extracts derived from fresh Datura metel leaves in comparison to similar extracts created from dry leaf material when tested against multiple bacterial species ${ }^{27}$. It is therefore possible that drying risks the loss of volatile compounds in the plant material, potentially lessening antibacterial potential. Regardless of the cause for differential antibacterial results between fresh and dried plant material, a sound investigation of a single plant may require testing of both. 
Following drying, plant parts are then often, but again not always, ground or pulverized. In the abovementioned studies, both Kuppusamy et al. and Kenny et al. powdered dried plant material using mechanical blenders, the former following this step with sieving of blended material through a $40 \mu \mathrm{m}$ mesh $^{31,44}$, whereas Voravuthikunchai and Limsuwan, using a similar approach, crushed dried plant material in a mechanical mortar ${ }^{43}$. The use of grinding or pulverization is presumably to increase surface area exposure and enhance extraction efficiency, which is defined below. This enhancement likely improves the chances of recognizing any antibacterial compounds potentially present within the plant material, by increasing the odds that these compounds will be present in the resulting extracts in high enough concentrations as to be detectable in antibacterial assays.

In the pharmaceutical sense, the creation of extracts from plant material refers to the separation of therapeutically active constituents, with simultaneous elimination of unwanted insoluble material through treatment with selective solvents ${ }^{45}$. However, extraction efficiency or extract yield, meaning the amount of extract produced per amount of starting material, may vary by plant part or by solvent used, or even when different methods are applied using the same solvent in treatment of the same plant material ${ }^{45}$. This potential variability was illustrated by Kaneria et al. for example, during an investigation of the antibacterial and antioxidant properties of five plants traditionally used as health supplements in Saurashtra folk medicine. A cold percolation method was used to create successive petroleum ether, ethyl acetate, methanol and aqueous extracts from a part(s) of each plant, and it was found that extract yield variability resulted not only among the five plants, but also between parts of individual plants ${ }^{4}$. Therefore, some 
trial and error may be unavoidable in efforts to optimize extract yields from a plant or plant part(s) selected for study.

Solvent choice and the methods employed in the treatment of plant material with selected solvents vary greatly. Additionally, solvent to plant material ratios are not standardized, or even discussed in the reviewed literature, thus once again, a trial and error method may be necessary to determine at what ratio most efficient extraction yields are achieved. Commonly selected solvents appearing in the literature include acetone, ethanol, ethyl acetate, n-hexane, dichloromethane, methanol, water, n-butanol, petroleum ether and hydro-alcoholic mixtures, though additional solvents appear as well. Akeel et al., for example, used sodium acetate buffer and sodium phosphate citrate buffer, the latter at six separate $\mathrm{pH}$ values, in the extraction of peptides and proteins from seeds of six plant species used in traditional medicine ${ }^{35}$. Alternatively, Roy et al. used chloroform and chloroform with added hydrochloric acid in chemical extractions of the medicinal herb Andrographis paniculata, claiming that this plants metabolites are known to be extracted at higher yields in more acidic solvents ${ }^{46}$.

Given the number of solvent possibilities, it would once again be reasonable, at least initially, to attempt to replicate the solvent choice, if not the entire extraction methodology, used in traditional folk medicine or phytotherapy for the specific plant(s) under study ${ }^{25}$. For example, if the plant of interest is typically prepared and administered as a tea (water solvent) or tincture (alcohol solvent), it would be reasonable to perform extractions using these same treatment methods and solvents to best refute or support claims of therapeutic value. In the screening of twelve northwestern Argentinian plants used in folk medicine, Soberon et al. for example, prepared infusions, decoctions and 
tinctures in accordance with traditional practices, and found that both the aqueous and alcoholic extracts of Tripodanthus acutifolius demonstrated antibacterial efficacy against several strains of bacteria comparable to those of commercially available antibiotics ${ }^{25}$.

There are also a number of different methods in the reviewed literature by which solvents have been applied to plant material in the creation of extracts, with most if not all investigations relying on a single method. Again however, there is little rationale provided by the authors to justify the application method selected. Some methods are very obvious or straightforward. Using Voravuthikunchai and Limsuwan again as an example; crushed Thai medicinal plant material was simply soaked in $95 \%$ ethanol for 7 days at room temperature ${ }^{43}$. In a similar approach, Aqil et al. soaked powdered material from four Indian medicinal plants in a 70\% ethanol solution for 8-10 days, stirring the mixture every ten hours ${ }^{10}$. In a slightly more complicated approach, Regazzoni et al. stirred dried and minced Rhus coriaria leaves in cold water bubbled with nitrogen gas, claiming this helps avoid polyphenol oxidation ${ }^{47}$.

Simple boiling or other heat-based treatments appear in the literature as well. Stanojevic et al. for example, created an aqueous extract from dried and ground Salvia officinalis leaves by cooking in a water bath at $80^{\circ} \mathrm{C}^{48}$, while Ganie et al. used a Soxhlet extractor at $60-80^{\circ} \mathrm{C}$ to produce a methanol extract from powdered Arnebia benthamii plant material $^{49}$. Though these heat-based methods may potentially increase extract yields in comparison to non-heat requiring methods, they may also risk denaturing or destroying heat-labile antibacterial proteins or compounds. The length of heat exposure may be a deciding factor in some cases. Kousha and Ringo for example, reported the stronger efficacy, against some bacterial strains, of aqueous extracts created from 
Heracleum persicum and Heracleum mantegazzianum plant material which were prepared by 2 or 24 hours of boiling, in comparison to an identical extract which was not subjected to any heat-based treatment. Yet in several comparisons, extracts derived from 2 hour-boiling treatments demonstrated more antibacterial potency than those subjected to 24 hours of treatment ${ }^{36}$. It is therefore possible in this study that while 2 hours of boiling may have enhanced the extraction of an antibacterial compound(s) in comparison to the non-heat-based treatment, 24 hours of boiling resulted in the subsequent degradation of these compounds. Thus, the application of heat-based methods of extraction may in some cases result in a tradeoff between extract yield and the antibacterial potency or efficacy of the extract.

In one interesting comparative study of techniques, Kothari performed extractions from seeds of five plants (Annona squamosa, Manilkara zapota, Phoenix sylvestris, Syzygium cumini and Tamarindus indica) using water, methanol or ethanol solvents, and employing five different treatment methods: Soxhlet extraction, ultrasonication, continuous shaking at room temperature, and microwave extraction with or without intermittent cooling. Though various attributes of the created extracts were measured, it was the Soxhlet method that was concluded to be the best method in terms of extract efficiency. However, other methods were reported as resulting in extracts with superior antibacterial activity. Thus, the authors concluded that no single method is likely superior for the extraction of all types of bioactive metabolites ${ }^{45}$. It is therefore unfortunately very likely that a good deal of trial and error, combining the same starting plant material with various preparation methods, solvents, and treatment methods, may be necessary to detect the antibacterial properties of the plant under study, assuming they 
exist. Again, as such trial and error may add substantially to workload and cost, reference to the preparation and treatment methods applied in traditional medicine for the plant under study may help guide successful strategies.

The creation of an extract solution through the treatment of plant material with a solvent(s) may be achieved using independent treatment, sequential treatment, or by fractionation of pre-existing solutions created using other solvents. Independent treatment involves the application of a single solvent to a single sample of plant material. This will likely provide the greatest extract yields, though these results are likely to vary by solvent, as each solvent will likely fail to extract compounds of polarity greatly dissimilar to that of its own. Water, being the universal solvent, constitutes the most logical starting choice for the chemical extraction of plant material, as compounds found therein are very likely to be soluble in an aqueous environment. However, solvents of intermediate polarity, such as alcohols, may enhance the extraction of compounds possessing both polar and non-polar moieties. Methanol, for example, has been claimed to be superior to water, ethanol and hexane in the extraction of antibacterial compounds $^{2,50}$. A potential advantage of an independent treatment strategy is that if solvents of different polarities are applied independently to separate samples of the same plant material, a collection of solutions is created that likely encompasses a large number of the compounds present in the starting material. Jesionek et al. for example, used methanol, ethanol and ethyl acetate to create multiple extracts from dried plant material of each of three species: Sambucus nigra flos, Melisa officinalis and Viola tricolor ${ }^{51}$. However, such a strategy increases the amount of starting plant material required, and has the additional potential disadvantage of resulting in final extracts containing large 
numbers of compounds for subsequent identification should an extract(s) demonstrate antibacterial activity.

Sequential treatment is the application of multiple solvents to a single sample of plant material. Rocha-Gracia et al., for example, treated samples of eighteen Mexican plant species, with one or more solvents each, using the following polarity-based order of solvent application: n-hexane, acetone, ethyl acetate, methanol, ethanol, and a methanolwater mixture $^{20}$. Similar to the independent treatment of multiple plant material samples using different solvents, a sequential treatment strategy such as this may be used to extract compounds of widely differing polarities. Sequential treatment also provides the advantage over independent treatment of requiring only a single sample of plant material. However, if more than one of the solvents being used in sequential treatment withdraws a compound(s) possessing antibacterial activity from the plant material, and these solvents do so with different efficiencies, it is conceivable that the compound(s) of interest could be dispersed among the final extracts in low enough concentrations to the point where detection of antibacterial activity could be hindered.

Fractionation is accomplished by the addition of a different solvent to a preexisting extract solution. Lee et al. for example, in testing seven edible plants from Thailand in efforts to identify alternative antibiotics for feed additives, first fractionated methanol extract solutions using n-hexane/water mixtures, then further partitioned the resulting aqueous layers by means of sequential treatments with chloroform-water, ethyl acetate-water and butanol-water mixtures ${ }^{34}$. Fractionation allows for the enhanced partitioning of compounds of slightly different polarities into separate solutions in comparison to independent or sequential treatment, and reduces the number of 
compounds for identification. However, though this enhanced allocation may improve the chances of detecting a compound(s) with antibacterial activity by increasing its concentration in a single extract solution, the fractionation of every solution created by independent or sequential treatment of a plant material sample is likely to increase cost and workload. Therefore, it may be desirable to reserve fractionation for extracts which have already displayed antibacterial activity.

Additional factors complicating the extraction process, which are nearly impossible to predict, are those of antagonistic or additive effects between phytocompounds in the same crude extract. For example, in investigating the antimycobacterial effects of compounds found in Fructus Euodiae, Hochfellner et al. reported antagonistic effects between indoloquinazoline alkaloids and the quinolone alkaloid evocarpine, both of which were present in the original plant material ${ }^{52}$. It is therefore possible that if an antibacterial compound(s) exists within a plant, but is normally physically sequestered from an antagonistic compound(s) in another plant part, that the use of whole plants, or particular solvents in the creation of an extract may result in the antibacterial activity of the first compound being masked if the two are simultaneously withdrawn from the plant material. Conversely, it is also possible that a chosen strategy for extract creation may fail to simultaneously withdraw compounds which would otherwise act in concert to exert antibacterial effects. Once again, as exhaustive testing of these possibilities would mandate the physical separation of every compound present in the plant material, it would once again be advisable to consult the preparation methods used for the plant under study in traditional medicine for reference in planning initial experiments. 
Following solvent treatment of plant material, insoluble matter can be removed from the resulting solution, if so desired, using means as simple as the passage of the liquid through cotton, cloth or filter paper. Adwan and Mhanna for example, filtered water extracts made from plants obtained in Palestine using Whatman No. 2 filter paper under vacuum ${ }^{53}$. However, a single passage through materials such as these is unlikely to remove all insoluble matter. Perhaps for this reason, Motz et al. for example, first vacuum filtered methanol extracts of Impatiens capensis using a Whatman Grade 1 filter, then repeated the process using a Whatman Grade GF/F filter ${ }^{54}$. However, multiple filtrations increases the amount of extract likely lost to absorption by the filter material. Centrifugation, alone or followed by filtration of the supernatant, may provide a viable alternative. Tolmacheva for example, in the creation of extracts from Eastern European medicinal plants, centrifuged aqueous or ethanol solutions at 1000 times g for $10 \mathrm{~min}$, then passed the supernatants through $0.2 \mu \mathrm{m}$ polyethersulfone syringe filters to ensure the sterility of the final products ${ }^{55}$. Dried extracts can also be sterilized. Chatterjee for example, subjected dried Vangueria spinosa extracts to UV exposure for 24 hours, then streaked samples on nutrient agar plates to monitor for contamination ${ }^{56}$. However, it is possible that such irradiation may inadvertently cause photochemical reactions to occur within the extract.

Following filtration, excess chemical solvent may need to be removed from the extract solution. Removal of excess solvent not only increases the concentration of the plant compounds in the final solution, but is also necessary if the solvent is insoluble in the media intended to be used for antibacterial testing, or if the solvent itself is toxic to bacteria. Exposure to an open air environment, the use of a laminar flow hood, and 
biosafety cabinets represent simple options for solvent removal. Alabri et al. for example, evaporated methanol, hexane, chloroform, ethyl acetate and butanol solvents from Datura metel extracts by simply allowing them to dry in a fume hood ${ }^{27}$. However, at room temperature, these simple methods are likely to be time consuming. Without the application of heat, water or water-based solvents in particular, can be very difficult to remove. Lyophilizing of these extracts provides a good alternative method, if available, and use of this technique appears numerous times in the reviewed literature. Yildirim et al. for example, in the creation of extracts from Turkish medicinal plants, lyophilized a filtered aqueous solution with a freeze-dryer at $-65{ }^{\circ} \mathrm{C}$, while for alcoholic filtered solutions, the solvents were first removed via rotary evaporation under vacuum at $60{ }^{\circ} \mathrm{C}$, following which these were dissolved in distilled water and also lyophilized ${ }^{28}$.

Heat-based methods of solvent removal however, such as the use of rotary evaporation, a speed vacuum concentrator, or even a water bath, similar to heat-based extraction methods, may damage heat-sensitive compounds. Perhaps to minimize this heat degradation risk, Ocheng et al., in creating extracts from Ugandan plants used in the treatment of oral/dental diseases, first removed the solvents from filtrated hexane and methanol solutions using rotary evaporation until volumes of approximately $50 \mathrm{ml}$ were reached, then dried the remaining solutions using an oven at $40-50{ }^{\circ} \mathrm{C}^{42}$. It is reasonable to expect that during either solvent application or removal, exposure to temperatures greater than those to which the plant under study would normally be exposed in a natural environment might result in at least some level of chemical degradation within the plant material, and control experiments may be useful in exploring this possibility. 
Two unique methods of extract preparation appearing in the reviewed literature meriting discussion are those of essential oil preparation and supercritical fluid extraction. Obtained by steam distillation of plant material, often using a Clevenger-type system, essential oils are mixtures of volatile compounds which often carry the aroma and scent of the plant ${ }^{7}$. Liquid, limpid, and mostly colorless ${ }^{18}$, these oils can subsequently be dehydrated through the use of various drying agents. Salehi et al., for example, used anhydrous sodium sulfate in the removal of water from an essential oil prepared from the hydrodistillation of Ziziphora clinopodioides subsp. rigida plant material ${ }^{57}$. By relying on an aqueous solvent, this method has the advantage of producing an extract free from organic solvents. However, as high temperatures are applied, as discussed above, there exists the potential for thermal degradation of plant peptides and compounds ${ }^{7}$. Additionally, essential oil production has the shortcoming of being unable to extract metabolites of large molecular mass ${ }^{7}$.

By comparison, supercritical fluid extraction subjects solvents to higher than critical temperatures and pressures higher than critical pressures, giving the solvent a high density, yet allowing it to retain its diffusion ability. As a result, the solvent can more easily penetrate the plant material. Pressure reduction then converts the solvent to a gas, separating it completely from the liquid or semiliquid extract, providing a solventfree sample ${ }^{7}$. Further, thermal degradation of peptides or other compounds can be avoided by using carbon dioxide for the extraction of plant material, which requires temperatures of only about $40^{\circ} \mathrm{C}^{7}$. Additional advantages of SFE, similar to microwave assisted or ultrasonic assisted extraction, are the reduction in organic solvent consumption and relative minimal sample degradation ${ }^{45}$. Thus, as it potentially offers 
good yields and can circumvent the problem of thermal degradation of plant compounds, this second method appears to have advantages over more commonly used chemical extraction methods, and may very well see greater favor of usage in the future.

Though not completely analogous to extracting antimicrobial compounds from plants, another recently developed technique which appeared several times in the reviewed literature is the application of plant extracts in the biosynthesis of metallic nanoparticles, which has become an important branch of this field ${ }^{58}$. These synthesized nanoparticles are believed to have antibacterial properties, and may prove useful in topical applications, such as the coating of medical devices for sterility purposes ${ }^{59}$. Ionic silver, for example, is believed to be capable of causing cell death by inactivating bacterial enzymes, inhibiting DNA replication and damaging the bacterial cell membrane $e^{60}$. These antibacterial properties can be enhanced by using plant extracts as capping ligands, which are believed to inhibit aggregation by binding to the nanoparticle surface, thereby enhancing their water solubility and stability ${ }^{60}$. The advantages of nanoparticle formation using plant extracts include being simple, cost-effective and easily scaled up to large production, as well as reducing waste products, improving efficiency, and being eco-friendly ${ }^{58,59}$. Therefore, while not directly applicable to the treatment of infectious diseases, nanoparticle production from plant extracts may help reduce the spread of pathogens via nosocomial means, while also averting some of the negative environmental and financial consequences of synthetic bactericidal use.

\section{Antibacterial Testing}

The ultimate goal of antibacterial testing in plant studies is the determination of the minimum inhibitory concentration (MIC), the minimum extract concentration at 
which growth of a specific strain of bacteria is halted, and/or the minimum bactericidal concentration $(\mathrm{MBC})$, the minimum extract concentration at which a specific strain of bacteria is killed. Though there is little standardization in terms of the antibacterial assays employed in the testing of plant extracts, the techniques appearing in the reviewed literature are very similar to those used in synthetic antibiotic evaluations. The KirbyBauer test, or disc diffusion assay for example, is the standard antibacterial assay in widest use $\mathrm{e}^{23}$, and is also often used in plant extract testing in the literature. Extracttreated discs are placed on solid media plates which have been inoculated on the entirety of their surfaces with bacterial cultures. During incubation, as the bacteria attempt to grow to complete confluence, the extracts diffuse from the discs, and those extracts containing antibacterial compounds create clearings, or zones of inhibition, surrounding the discs. The antibacterial potency of an extract can be estimated by comparison of the size of its zone of inhibition to that created by a synthetic antibiotic control. The disc diffusion method therefore provides a relatively easy and inexpensive means of testing multiple extracts against a single pathogen at one time.

However, the disc diffusion method has several potential drawbacks. Obtaining zones of inhibition uniform in diameter necessitates even impregnation of the disc with the extract ${ }^{21}$, which is not necessarily easily accomplished by hand. Soaking of the disc in an extract prior to use may represent a preferable alternative. However, to allow for disc soaking, the extract will need to be in liquid form, or re-suspended in solvent if it is dry. The choice of solvent for extract suspension is important in disc diffusion assays, as the solubility of the extract, or the solvent in which it is suspended, can affect the rate of diffusion in the growth media being used, thereby influencing the distance travelled from 
the disc $\mathrm{c}^{4,10,22}$. This can potentially diminish the size of a zone of inhibition if the extract or solvent are not completely soluble in the growth media used for the disc diffusion. The disc diffusion assay is therefore not suited to antimicrobial compounds or plant extracts which are insoluble or scarcely soluble in water ${ }^{61}$.

Further, the initial bacterial inoculum level ${ }^{21}$, the growth rate and metabolic activity of the microorganism being assayed in the media used ${ }^{4}$, and the temperature at which the assay is conducted ${ }^{22}$, may similarly prejudice the size of zones of inhibition, as an extract's antibacterial efficacy may, for example, be masked by overwhelming inoculum levels or particularly virulent growth, both of which may be temperaturedependent. It may therefore be best to reference existing literature pertaining to the specific strain(s) of bacteria under study to mimic bacterial inoculum levels and temperatures employed in studies of other extracts or synthetic antibiotics. As a result of these drawbacks, the disc diffusion method is considered an essentially qualitative, nonstandardized method, primarily useful only for the preliminary screening of multiple samples in a single assay ${ }^{2,22}$.

An essentially identical method which appears in the reviewed literature is the agar well diffusion method, wherein uniform holes, or "wells", are punched into solid growth media and filled with liquid extract samples. In the antibacterial testing of extracts and fractions from ten species of Indian medicinal plants, Aqil et al. for example, first spread test organism inoculums on the surface of Muller-Hinton agar plates, then punched $8 \mathrm{~mm}$ wells in the media, filled these wells with $100 \mu \mathrm{l}$ of plant extracts each, and incubated the plates overnight before measuring zones of inhibition ${ }^{62}$. However, bacterial inoculums can also be mixed directly into the media. This direct-mixing 
method was used by Khan et al. for example, in testing the antibacterial activities of extracts created from Gloriosa superba rhizomes. Bacterial cultures were diluted in cooled molten agar prior to plate pouring, and wells were then eventually dug into the solidified, bacteria-containing media ${ }^{19}$. While the well-punching method eliminates concerns regarding the uneven distribution of the extract in treated discs, extract solubility and diffusion rates in the media, as well as initial bacterial inoculum levels, virulence and temperature, still represent variables capable of influencing results.

An alternative approach is to pre-treat the media surface or the media itself with the plant extract under study before applying the bacterial inoculum. For example, using the slanting tube method, wherein media is solidified in a vessel tilted at an angle to increase surface area, Jiang et al. treated solidified potato dextrose agar growth media with Blumea balsamifera "volatile oil" samples prior to inoculation with plant pathogenic fungi, citing the minimal oil concentration capable of preventing visible growth as the $\mathrm{MIC}^{63}$. By contrast, Weckesser et al. mixed plant extracts and isolated compounds directly into Mueller-Hinton and Wilkins-Chalgren agar prior to inoculation with bacterial strains and yeasts of dermatological relevance, though an almost identical criterion for MIC evaluation was used ${ }^{64}$. However, mixing plant extracts in the media, while potentially eliminating the diffusion problems inherent to disc usage or wells, only allows for the detection of the presence or absence of bacterial growth, with no quantitative zone of inhibition measurement, with results still susceptible to bacterial inoculum level, virulence and temperature influences.

The microbroth dilution method, in which the growth of bacteria in extract-treated liquid media is measured, is arguably the strongest of the described techniques for the 
antibacterial testing of plant extracts. Provided the extract or the solvent in which it has been suspended is soluble in the liquid media, extract homogeneity should be easily achieved. Further, more accurate quantitation of results is possible using this method, either through visual, colony counting, or spectroscopic means. For example, growth inhibition in a microbroth sample can be easily assessed by visual inspection of the sample for a lack of cloudiness or turbidity indicative of bacterial growth, though this is a somewhat subjective measurement. Antibacterial efficacy can also be quantified by diluting and plating extract-treated samples on nutrient agar plates and counting colony forming units after incubation, a method used for example by Wojnicz et al. in examining the effects of extracts derived from medicinal plants used in urinary tract infections on a clinical Escherichia coli strain $^{65}$. However, this method is likely to be time consuming. Spectroscopic means however, such as microplate readers, can be used to provide quick and specific quantification of bacterial growth. The measurement of the optical density of a culture sample at a wavelength of $600 \mathrm{~nm}$ for example, indicates the amount of light scattering by bacterial cells, which correlates to cell density or growth ${ }^{59}$.

However, when using the microbroth dilution method, the effect of initial bacterial inoculum levels and temperature on experimental results remain potential concerns. Additionally, a common problem encountered when using the microbroth dilution method is that plant extracts may possess color ${ }^{22}$. Coloring in an extract can complicate the evaluation of test samples when relying on growth detection methods such as visual inspection for turbidity or spectroscopic means. For example, when visually inspecting a sample, coloring or particulate matter in an extract may make it difficult to discern turbidity attributable to bacterial growth from that resulting from these extract 
properties. Similarly, when using spectroscopic means to evaluate microbroth test samples, initial optical density levels may be significantly inflated by the addition of extract, in comparison to the optical density attributable to the media and the bacterial inoculum. It will therefore be difficult to detect bacterial growth in these extract-treated samples until they reach optical density levels surpassing those of the initial test mixtures. Therefore, when using spectroscopic means, to account for the optical density of the extracts, as well as the media and the bacterial inoculum, the optical density of extracttreated samples should be measured after extract addition, but prior to incubation, so that these initial values can be subtracted from post-incubation measurements. Still, in comparison to solid-media based methods, the microbroth dilution method allows for more accurate quantification of MIC levels, the simultaneous screening of combinations of different plant extracts and bacterial strains in the same sample, and is more economical in terms of time and resources required ${ }^{61}$.

Colorimetric indicators, such as tetrazolium salts, which living bacteria convert to colored formazan derivatives, can be added directly to extract-treated samples, and also allow for a quantifiable method of detecting bacterial growth ${ }^{61}$. Eldeen et al. for example, applied the salt p-iodonitrotetrazolium violet to microbroth dilution bacterial samples treated with extracts derived from trees used in South African traditional medicine, interpreting the appearance of a red color as indicative of bacterial growth ${ }^{1}$. Alternatively, Tekwu et al. added Cameroonian plant extracts and bacterial cultures to Mueller Hinton Agar containing glucose and the $\mathrm{pH}$ indicator phenol red, which turns yellow upon acidification of the media in the event of metabolism of the added sugar by living bacteria ${ }^{2}$. The detection of ATP in living cells may provide another means of 
detecting bacterial metabolic activity, and can be assayed using the reagent Bac-Titer Glo $^{\mathrm{TM}}$ with a plate reader ${ }^{61}$, a method used by Mekinic for example, in the investigation of the antibacterial properties of selected Lamiaceae species ${ }^{66}$. Other colorimetric indicators appearing in the reviewed literature include the tetrazoium salts $\mathrm{TTC}^{61}, \mathrm{MTT}^{51}$, and Nitro blue tetrazolium ${ }^{67}$ and the dye resazurin ${ }^{11,68,69}$ (also known as Alamar Blue).

However, the use of colorimetric indicators, in the reviewed literature at least, is done for MIC determination. A lack of metabolic activity demonstrated by colorimetric indicators does not necessarily imply that the treated cells have been killed. Additional testing is necessary to discern whether plant extract antibacterial effects, if present, are bacteriostatic or bactericidal in nature, or at what concentration effects may become bactericidal. Bactericidal effects can be confirmed by a lack of observable growth after transfer of extract-treated samples to fresh media. For example, in examining the antibacterial activities of Humulus lupulus components against bacterial strains believed to play a role in the etiology of acne vulgaris, Yamaguchi et al. plated microbroth dilution samples displaying no visible growth onto agar plates, then incubated these plates and observed them for lack of bacterial growth in order to determine bactericidal concentrations $^{70}$. Similarly, Weckesser et al. again for example, first mixed plant extracts or single plant compounds directly into agar plates, inoculated and incubated these plates, then excised and streaked inoculation spots displaying no visible growth onto fresh plates for MBC determination, choosing the lowest MIC concentrations failing to demonstrate visible growth after transfer ${ }^{64}$. If a plant extract is found to be bactericidal, time-kill assays, using liquid media, are a useful means of quantifying the bactericidal potency of the extract. Transfer of extract-treated samples to fresh media at various time points 
allows for a measurement of the length of exposure necessary for lethal effects to be exerted. Leandro et al. for example, in investigating the antibacterial efficacy of the compound dehydroabietic acid, derived from Pinus elliottii, removed aliquots from treated samples of Staphylococcus epidermidis at time points of 30 minutes, 6, 12 and 24 hours, then serially diluted, plated, and observed these samples for visible growth after incubation $^{68}$.

As another consequence of the lack of standardization in this field of research, there is a wide discrepancy in the reviewed literature with regards to the working concentrations of extracts used in antibacterial assays. In the aforementioned evaluation of Salvia officinalis extracts, Stanojevic et al. for example, reported testing concentrations as high as $40 \mathrm{mg} / \mathrm{ml}^{48}$, while Hussain et al. assayed concentrations as high as $100 \mathrm{mg} / \mathrm{ml}$ in the evaluation of extracts from plants used in traditional medicine in Pakistan ${ }^{71}$. Remarkably, Ugoh et al. reported testing concentrations as high as $500 \mathrm{mg} / \mathrm{ml}$ when evaluating the effects of Khaya senegalensis stem bark extracts on a subspecies of Salmonella enterica ${ }^{3}$. It is important to consider that besides the potential experimental influences described earlier, such as inoculum level or temperature, antibacterial assay results for plant extracts may also be affected by factors such as salt formation, precipitation, autofluorescence, or antioxidant properties of the extracts ${ }^{22,61}$. Consequently, the use of such extreme concentrations as those described above increases the probability that one or more of these potential influences will adversely affect assay results. However, it may be possible to account for at least some extract property influences experimentally. When investigating the potential of several organic acids and plant extracts in food preservation applications, Over et al. for example, in an effort to 
preclude $\mathrm{pH}$ effects as a contributing factor to antibacterial efficacy, included control samples with $\mathrm{pH}$ values titrated to match those of organic acid-treated samples ${ }^{72}$.

There is also an apparent lack of agreement as to what constitutes a desirable or relevant MIC value for a plant extract, which likely contributes to the use of the extreme working concentrations described above. Prior to sub-fractionation or the isolation of an individual compound(s), the crude state of the initial extract and the potentially low concentration of the compound(s) responsible for its antibacterial activity ${ }^{46}$, if present, will likely result in high experimental MIC values in comparison to those of synthetic antibiotics. Consequently, acceptable or meaningful MIC levels for extracts are subjective, as the concentration of any antibacterial compound(s) is unknown. It has been suggested that crude extracts and essential oils demonstrating antibacterial effects at or below concentrations of $100 \mu \mathrm{g} / \mathrm{ml}$ are promising candidates for potential pharmaceutical use, while for an isolated compound, this number drops to $10 \mu \mathrm{g} / \mathrm{ml}$, and preferably less than $2 \mu \mathrm{g} / \mathrm{ml}^{40,68}$.

Synergistic relationships among extracts, or between extracts and synthetic antibiotics, are explored numerous times in the reviewed literature. The exploration of extract/extract combinations is justified when it is remembered that traditional healers often use combinations of plants in disease treatment ${ }^{56}$. For example, Aqil et al. reported synergistic antibacterial effects among four Indian medicinal plant extracts, as well as between these extracts and synthetic antibiotics, in testing against a number of clinical MRSA strains ${ }^{10}$. Similarly, Adwan and Mhanna reported reductions in synthetic antibiotic MIC values against Staphylococcus aureus clinical strains when applied in combination with aqueous extracts of plants obtained in Palestine ${ }^{53}$. Chatterjee et al. as 
well, reported an ethanol leaf extract of Vangueria spinosa as lowering the MIC values and enhancing the time-kill assay results of the synthetic antibiotics doxycycline and ofloxacin when combined in the treatment of numerous bacterial strains ${ }^{56}$.

Mutagenicity, toxicity, and stability testing of plant extracts are not always included in the literature articles reviewed, but are certainly necessary components of a thorough investigation. As noted by Eldeen et al., there exist claims of plants used in traditional medicine having displayed mutagenic effects in in vitro assays. Therefore, in the aforementioned screening of extracts derived from trees used in South African traditional medicine, this group employed the Ames test with a strain of Salmonella typhimurium, considering the extracts "active" if the number of revertant colonies after incubation doubled that of the untreated control ${ }^{1}$. Direct toxicity of plant extracts towards eukaryotes can be tested using numerous cell types and animals. Owais et al. for example, evaluated the safety of Withania somnifera extracts by monitoring for lysis of treated and incubated human erythrocytes using hemoglobin absorbance readings at 600 $\mathrm{nm}^{73}$. By comparison, Miceli et al. tested for Borago officinalis and Brassica juncea extract lysis of sheep erythrocytes by observing for the failure of treated and incubated cells to form a pellet after centrifugation ${ }^{13}$. Using in vivo methods, in the aforementioned Jiang et al. study of Blumea balsamifera, "volatile" oil-treated prawn larvae were evaluated by microscopy after cultivation to determine lethal effects ${ }^{63}$. Alternatively, Toyang et al. tested the toxicity of a Vernonia guineensis extract using a slight modification of the 2000 World Health Organization method for assessing the acute toxicity of medicinal plants, in which the extract was administered to Sprague-Dawley rats by oral gavage, and the animals monitored for behavioral changes for a period of 
seven days prior to surviving specimens being euthanized and necropsied ${ }^{74}$. Finally, use of plant extracts in different applications also requires that the extract be quite stable ${ }^{30}$. To evaluate stability, Miceli et al. again for example, tested extracts before and after one year of storage at room temperature, $4^{\circ} \mathrm{C}$ and $-20^{\circ} \mathrm{C}$, and reported the loss of antibacterial efficacy versus the seven bacterial strains showing greatest sensitivity prior to extract storage for all temperature treatments assessed ${ }^{13}$.

If plant extracts are being considered for use in agricultural or food preservation applications, such intentions necessitate additional evaluations. Previous research has demonstrated that the intrinsic properties of food, including fat, protein, water or salt content, water activity, $\mathrm{pH}$, the presence of other additives, antioxidants or preservatives, partition coefficients, and extrinsic determinants such as processing and storage temperatures, storage atmosphere, vacuum packaging, air and gas levels, as well as target microorganism characteristics and the interactions between these factors, can influence bacterial sensitivity to antibacterial compounds and may require higher extract concentrations in comparison to those demonstrating efficacy in in vitro assays ${ }^{18,33}$. These influences may be the result of the binding or inactivation of antibacterial compounds by food components or additives, or changes in extract solubility or phase distribution ${ }^{13,33}$. Another possibility is that the greater nutrient availability in food compared to that in growth media may result in faster bacterial cell damage repair ${ }^{33}$, thus diminishing potential antibacterial effects. For example, Miceli et al. tested their Borago officinalis and Brassica juncea aqueous extracts using three food model systems (meat, fish and vegetable broths), and reported that in vitro concentrations demonstrating antibacterial efficacy failed to do so in these models. However, these extracts did show 
antibacterial effects when applied at ten times the levels used in the in vitro assays ${ }^{13}$. Similarly, Klancnik et al. evaluated the antibacterial potency of commercial rosemary extracts versus L. monocytogenes and E. coli in food models of meat, vegetable, and dairy products, and reported that the type of food model tested influenced MIC results, with higher values registered for high-protein and high-fat meat and dairy products, as opposed to vegetable models ${ }^{33}$. In an encouraging report of synergistic effects, Abdollahzadeh et al., in testing various plant essential oils and extracts for antibacterial activities versus Listeria monocytogenes, found the essential oil of thyme, alone and in combination with the antimicrobial peptide nisin, to be effective in inhibiting the growth of this pathogen in minced fish samples. However, the authors also noted that since nisin, when tested alone, was more effective in a model of cooked fish in comparison to the minced fish model, that the manner in which the food model is prepared may influence results ${ }^{18}$, thus making direct comparisons of MIC values among similar studies difficult.

A complicating factor to the use of plant extracts in agricultural or food preservation applications is the potential effect(s) they might have on the organoleptic profile of treated products ${ }^{13}$. Plant extracts may change food flavor or smell ${ }^{13,18}$, possibly making treated crops less appealing to agricultural animals, or making treated finished food products less palatable to human consumers. Also, selective activity against particular strains of bacteria, without broad spectrum antibacterial effects, may be desirable if the extract is intended for use as a preservative in foods already containing bacterial cultures, such as yogurt or cottage cheese. The selective inhibitory action of sinapic acid, which has been identified as a component of Bassica juncea by Engels et al., 
in starter cultures, protective cultures, or probiotics for example, allows for the elimination of foodborne pathogens without growth or metabolic inhibition of the beneficial lactic acid bacteria present in these products ${ }^{75}$.

Finally, without in vivo testing using animal or human subjects, the former appearing rarely in the reviewed literature, the latter appearing not at all, in vitro results alone are limited in their predictive ability of the usefulness of plant extracts as clinical antibiotics. In the evaluation of plant extracts as potential oral antibiotics for example, it should be remembered that the gastrointestinal system significantly affects the final bioavailability of conventional medicines ${ }^{38}$, and that this is of particular relevance given that oral consumption is perhaps the most prevalent route of administration in traditional medicine. The human stomach has a pH level of 1-2, while the small intestine contains numerous additional digestive enzymes and has a $\mathrm{pH}$ of roughly $5.1-7.5^{38}$. It is difficult to predict how these environments will affect the final bioavailable concentration(s) of an antibacterial compound(s) present in an extract ${ }^{53}$. However, some information regarding the potential effects of the digestive system can be obtained using simulated gastrointestinal fluids, which can be prepared and used in in vitro antibacterial assays. For example, using the 1990 U.S. Pharmacopoeia, Vermaak et al. created simulated gastric and intestinal fluids, and found that Tarchonanthus camphoratus extracts lost some of their antibacterial activity after exposure to simulated gastric fluid, and completely after exposure to simulated intestinal fluid, suggesting possible degradation of an antibacterial compound(s) in these environments. Interestingly however, they also reported that one Agathosma betulina extract became active only after exposure to simulated intestinal fluid, suggesting the possible activation of an antibacterial 
compound(s) in this simulated environment. The authors therefore concluded that in vitro screening results may in fact both overestimate or underestimate in vivo antibacterial potential ${ }^{38}$.

Methods of Isolation and Identification

The isolation and identification of the compound(s) present in a plant extract which are responsible for its antibacterial activity are steps not routinely included in plant investigations in the reviewed literature. This may be the result, in some cases, of a lack of access to the necessary reagents or equipment for such analyses, while in other cases it is possible that the authors do indeed regard these plant investigations primarily as mere screening exercises, and leave such analyses for future studies. In any event, the isolation of the active compound(s) in an extract demonstrating antibacterial activity is necessary for a more exact determination of MIC and MBC levels. Identification of the compound(s) will reveal whether a novel antibiotic has been discovered, which may be of therapeutic value in combatting drug-resistant bacterial pathogens, or whether a previously identified naturally occurring compound has been found. However, it is possible that an already discovered, and even commercially available, naturally occurring compound may have never been previously recognized as having antibacterial properties.

Once again, as there again is no standardized protocol or preferred technique(s) for the isolation and identification of plant extract constituents, a wide variety of chromatographic, chemical, instrumental, and combinations of these methods appear in the reviewed literature. Though some of the more advanced chromatographic and instrumental methods are clearly superior in terms of providing a detailed account of extract composition, the cost of such equipment is potentially prohibitive. Yet there exist 
a variety of less expensive, readily available methods, capable of detecting compound classes or individual compounds within a plant extract. While an exhaustive discussion of the advantages and disadvantages of all these methods is beyond the scope of this written work, it is instead the author's intent to focus on making the reader aware of the numerous methods of isolation and identification currently used in the analysis of plant extracts.

Readily available methods for the detection of compound classes in a plant extract often involve simple chemical assays using reagents which can be applied directly to the extracts, or to extract fractions separated by chromatographic methods. Compound classes routinely identified in the reviewed literature using such methods, and which are believed in some cases to possess antibacterial activities, include alkaloids, amino acids and proteins, flavone derivatives such as flavonoids and flavonols, glycosides, iridoids, phenols and phenol derivatives such as phenolcarboxylic acids or proanthocyanidins, saponins, steroids or sterols, tannins, and terpene derivatives such as terpenoids or triterpenoids. Chemical methods employed in the reviewed literature for the detection of alkaloids in plant extracts for example, include colorimetric indicators such as Dragendorff's reagent ${ }^{31}$, Hager's reagent ${ }^{24}$, Mayer's reagent ${ }^{73}$, Wagner's reagent $^{73}$, and the Marquis reagent, which is composed of a mixture of sulfuric acid and formaldehyde 76,77. Similarly, the colorimetric indicator Ninhydrin can be used for the detection of amino acids or proteins ${ }^{76,78}$. The presence of flavonoids in plant extracts can be confirmed using a colorimetric method involving magnesium powder and hydrochloric $\operatorname{acid}^{78}$, with variations of this method appearing in the reviewed literature as well, and being referred to as the "Shinoda test" 24 "Shibata reaction" or "cyanide test" . A 
separate, very simple colorimetric method that has also been used for flavonoid detection involves the observance of a yellow color change after the addition of sodium hydroxide to a hydrochloric acid-containing extract, or the disappearance of this color following the addition of the acid to a sodium hydroxide-containing extract ${ }^{27,31}$.

The presence of glycosides can be confirmed using a colorimetric test known as "Legal's test", involving a pink to blood red color formation after extract treatment with dilute hydrochloric acid, followed by the addition of sodium nitroprusside in pyridine and methanolic alkali ${ }^{24}$. Alternatively, Owais et al., in the aforementioned study of Withania somnifera extracts, used a modification of the colorimetric "Keller-Killani" test method as a means of glycoside detection, a longer method involving lead acetate, chloroform, glacial acetic acid, ferric chloride and sulfuric acid reagents ${ }^{73}$. The presence of phenols can be confirmed by the appearance of a blue or green color $^{31}$, or a bluish black color ${ }^{24}$ following the addition of a solution of ferric chloride to a plant extract, or by a red color following the application of Fast blue B reagent ${ }^{76}$.

Alternatively, the detection of saponins or saponosides actually requires no chemical treatment, and can be demonstrated by the observance of foaming after shaking of the plant extract ${ }^{5,27,31}$, though a "blood reagent" can be used for chemical detection ${ }^{25}$. Steroids can be detected in plant extracts using variations of a colorimetric method involving chloroform and sulfuric acid known as "Salkowski's test", Burchard test", a slight variation which includes the use of acetic anhydride ${ }^{24,31,79}$. The Libermann Burchard test can also been used to detect sterols and triterpenes ${ }^{5}$. Tannins can also be detected using this method ${ }^{27}$, or by the observation of a bluish or greenish black color following the addition of ferric chloride to an extract ${ }^{31}$. Srinivas and Reddy 
used a method wherein the formation of a white precipitate upon Pedalium murex extract mixture with a sodium chloride-containing gelatin solution indicated the presence of tannins ${ }^{24}$. Similarly, Liao et al. also used a gelatin-based precipitation method for the detection of tannins in Polygonum capitatum extracts, which was followed by a vanillinhydrochloric acid-based method to identify tannin structural types ${ }^{78}$. Finally, terpenoids can be detected by observing for a green color formation following the addition of a copper acetate solution to an extract suspended in water ${ }^{24}$.

Quantification of the concentrations of some of these classes of compounds can also be accomplished using chemical methods coupled to some chromogenic or lightbased method, along with the use of a reference standard. Bobis et al. for example, used spectrophotometric measurement after the addition of an aluminum chloride solution to extracts created from nettle, basil, thyme, costmary and yarrow, along with a quercetin reference standard, for quantification of total flavone/flavonol levels ${ }^{80}$. The concentration of flavonol monomers in plant extracts can also be determined using a spectrophotometry-based method involving p-dimethylaminocinnamaldehyde, with epicatechin as a reference standard ${ }^{66}$. To quantify flavonoid content, a spectrophotometric method based on the successive addition of sodium nitrate, aluminum chloride and sodium hydroxide solutions to an extract can be used, with butylated hydroxytoluene or quercetin serving as reference standards ${ }^{31,80}$, as can a separate method involving spectrophotometric quantification and the appearance of a yellow color following the addition of ethanol, aluminum chloride and potassium acetate solutions to an extract, also using quercetin as a reference standard ${ }^{34}$. Total phenol or polyphenol content of plant extracts can be determined using the "Folin-Denis" method, also known 
as the "Folin-Ciocalteu" method, involving treatment of plant extracts with a reagent of the same name and a sodium carbonate solution, followed by spectrophotometric analysis with reference standards such as tannic acid, gallic acid or quercetin ${ }^{19,34,45,57,69,80-83}$. For the quantification of proanthocyanidins, Hori et al. for example, used the vanillin assay with $d$-catechin as a reference standard in the study of aqueous extracts of Azuki beans ${ }^{84}$. Alternatively, without spectrophotometry, the total tannin content of plant extracts can be measured using the Lowenthal method, which uses titration with potassium permanganate ${ }^{85,86}$.

Chromatographic methods are useful in the fractionation of plant extracts prior to efforts to identify their contents. By isolating, to different extents, the chemical constituents of an extract, these means of physical separation enhance identification, and such methods are often found to precede constituent analyses in the reviewed literature. Fractioning of an extract may be accomplished using as simple a method as paper chromatography. Nemereshina et al. for example, combined one and two-dimensional paper chromatography with separate solvent systems for the fractioning of aqueous extracts of Plantago and Veronica species, prior to tentatively identifying individual compounds using the characteristic fluorescence of reagent-treated spots ${ }^{85}$. Alternatively, Leandro et al. first used vacuum liquid chromatography to fractionate a "resin-oil" oil of Pinus elliottii with an n-hexane/ethyl acetate based solvent system of increasing polarity, followed by classic chromatography of the fraction demonstrating the greatest antibacterial efficacy, to eventually isolate the candidate compound dehydroabietic acid $^{68}$. Similarly, Joray et al. used vacuum liquid chromatography on a silica gel with a hexane-ethyl ether-methanol solvent gradient to fractionate an Achyrocline satureioides 
ethanol extract. The fractions demonstrating antibacterial activity were then subjected to successive column chromatography to produce sub-fractions, from which the candidate compound 23-methyl-6-O-desmethylauricepyrone was eventually isolated ${ }^{67}$. In the aforementioned study of Fructus Euodiae, Hochfellner et al. used solid phase extraction and elution with acetonitrile/water solvents, followed by semi-preparative high pressure liquid chromatography (HPLC) of selected resultant fractions, to isolate the compounds evodiamine and rutaecarpine ${ }^{52}$. By comparison, to collect hydrophobic fractions from extracts of Eastern-European medicinal plants, Tolmacheva et al. used reversed-phase column chromatography with an acetonitrile/water/trifluoroacetic acid elution mixture ${ }^{55}$. Using an extensive combinatorial approach, Liao et al., in the previously described study of Polygonum capitatum, employed macroporous resin column chromatography, $\mathrm{MCl}$ column chromatography and liquid column chromatography to fractionate an aqueousethanol extract ${ }^{78}$. Though it is technically not a chromatographic method, a dialysis treatment of dandelion root extracts was used in the aforementioned Kenny et al. study for fractionation according to molecular weight ${ }^{44}$.

However, in addition to providing for physical separation, chromatographic methods can also provide for a tentative means of identifying individual compounds. When plant extract samples are run in tandem with reference compound standards, retention times and absorption spectra can be compared for identification, as well as quantification, purposes. For example, in the previously described study by Mekinic et al., phenolic compounds in the sage, thyme, lemon balm, peppermint and oregano extracts were identified using HPLC, by comparing retention times and absorbance spectra with those of reference standards, and quantified by comparing peak areas with 
those of external standard calibration curves ${ }^{66}$. Similarly, Bobis et al., in addition to performing spectrophotometric measurements as described earlier, used HPLC with phenolic acid and flavonoid standards to identify and quantify these types of compounds in extracts of nettle, basil, thyme, costmary and yarrow ${ }^{80}$.

Thin layer chromatography (TLC) represents a simple chromatographic method that may be very well suited to the investigation of plant extracts for antibacterial properties, and its use appears several times in the reviewed literature. Similar to other chromatographic methods, reference standards can be used to tentatively identify compounds by comparing their distances migrated during physical separation, known as $\mathrm{R} f$ values, to those of the reference plate spots. Again using Srinivas and Reddy as an example, Pedalium murex extracts were separated using both thin layer chromatography and high pressure thin layer chromatography, with compounds tentatively identified by comparison of their $\mathrm{R} f$ values to those of standard values ${ }^{24}$. Also, chemical reagents for compound class identification can be applied directly to fractionated samples on the chromatographic plate. Bashir et al. for example, used several of the aforementioned chemical indicators in the form of sprays in assessing compound classes present in thin layer chromatography-fractionated green tea extracts ${ }^{76}$. Additionally, spots may be physically scraped or otherwise removed from the chromatographic plate, allowing for recovery of the fraction or compound(s) of interest ${ }^{25}$. Further, thin layer chromatography provides for the possibility of analyzing many samples in one reaction, requires limited sample pre-treatment in comparison to high pressure liquid chromatography, for example, and allows for the evaporation of solvents used as mobile phase components, making it ideal for bioassays ${ }^{51}$. 
The particularly suitability of thin layer chromatography to plant investigations comes from its use in a bioassay wherein completed gels are overlaid with growth media containing bacterial cultures. Zones of growth inhibition may then be observed directly atop plate spots, thus readily identifying the fraction(s) or compound(s) possessing antibacterial activity. Additionally, some of the chemical methods previously described for detection of growth inhibition can be applied directly to the plate spots for further confirmation of antibacterial activity. Aqil et al. again for example, first fractionated Indian medicinal plant extract samples on silica gel thin layer chromatography plates, then overlaid the developed plates with nutrient agar containing methicillin-resistant Staphylococcus aureus. After incubation, p-iodonitro-tetrazolium violet was applied to the plates to confirm the antibacterial activity of chromatogram spots surrounded by zone of inhibitions ${ }^{10}$. Similarly, in the aforementioned study of Argentinian plants, Soberon et al. also overlaid dried TLC plates in bacteria-containing media. Following incubation, the plates were covered with an MTT-buffer solution and incubated in the dark, after which the plates were observed for the appearance of yellow spots indicative of growth inhibition $^{25}$.

In comparison to chemical or chromatographic methods, advanced instrumental methods provide for a more definitive identification of the individual compounds present in a plant extract. However, these instrumental methods are usually preceded by or coupled to a chromatographic method to fractionate the extracts prior to analysis. In combination, comparison of chromatographic retention times and mass spectral results to those of reference standards recorded in pre-existing databases allows for a more definitive identification of individual compounds. LC-MS, or liquid chromatography- 
mass spectrometry, for example, combines the physical separation capabilities of liquid chromatography with mass analysis capabilities ${ }^{21}$, and this coupling has seen increasing use in the structural characterization of complex matrices such as plant extracts ${ }^{47}$. Combining gas chromatography (GC) with mass spectrometry, Rodrigues et al. characterized essential oils derived from Mentha cervina by comparison of GC retention indices to those of a standard hydrocarbon mixture, and GC-MS spectra to a home-made library constructed from laboratory-synthesized components, reference oils and commercially available standards ${ }^{39}$. Alternatively, Kuppusamy et al. compared gas chromatography-mass spectrometry results of various Commelina nudiflora extracts with those of standards using the National Institute of Standards and Technology database ${ }^{31}$. Finally, combining the use of internal and external databases, Salehi et al. identified individual compounds in the essential oil of the subspecies rigida of Ziziphora clinopodioides by first comparing GC-MS results to those of an internal reference mass spectra library and standard compounds, then confirming these results through comparison of retention indices to those of standard compounds and literature reports ${ }^{57}$.

Multiple other uses of chromatographic techniques in combination with or coupled to instrumental analytical methods appear in the reviewed literature as well. Chaweepack et al. for example, followed HPLC with nuclear magnetic resonance spectroscopy and mass analysis to first isolate and then identify trans-p-coumaryl diacetate extracted from a galangal extract ${ }^{87}$. Alternatively, Lu et al. combined ultraperformance liquid chromatography with mass spectrometry in the analysis of tea, Galla chinensis and rhubarb extracts $^{88}$, while Wojnicz et al. combined this chromatographic method with a quadrupole-time of flight mass spectrometry instrument in the analysis of 
various extracts derived from plants used to treat urinary tract infections ${ }^{65}$. In the aforementioned Kenny et al. study, flash chromatography, followed by liquid chromatography and solid phase extraction nuclear magnetic resonance, were employed in the analysis of dandelion root extract fractions, with further compound verification done using liquid chromatography-mass spectrometry ${ }^{44}$. Dadasoglu et al. used gas chromatography with a flame ionization detector for quantitative characterization of the essential oils of various Origanum species, followed by the use of gas chromatographymass spectrometry for compound identification ${ }^{89}$. Finally, in a somewhat unique and potentially very useful approach, Liu et al., in an investigation of Chinese plants used to treat snake bites, first partitioned the extracts demonstrating the greatest antibacterial efficacy directly into 96-well microplates. The individual well contents were then tested for antibacterial activity, and the results for each were correlated against their HPLC retention times. This allowed for identification of the compounds of interest, using HPLC coupled to high resolution mass spectrometry-solid phase extraction-nuclear magnetic resonance ${ }^{90}$. One advanced instrumental method that may prove to be of particular use in the evaluation of plant extracts was described by Regazzoni et al. in the previously mentioned study of aqueous extracts of Rhus coriaria, wherein flow injection analysis was coupled to high resolution mass spectrometry. According to the authors, this method provides the advantages, in comparison to a similar method using HPLC in place of flow injection, of requiring no chromatographic separation of extracts prior to analysis, having generally short analysis times, and needing no solvent consumption for liquid chromatography ${ }^{47}$. Therefore, by eliminating the need for coupling to a chromatographic method, this method may simplify plant extract analysis and compound 
identification. However, it is possible that there exist other advanced instrumental methods capable of more efficient or more precise analysis offering similar advantages, which did not appear in the reviewed literature.

Mechanisms of Action

Elucidating the specific mechanism of action by which a plant extract or compound exerts its antibacterial activity can help to predict its potential clinical or agricultural significance, either as a stand-alone antibiotic with a novel mechanism of action, or as an adjunctive therapeutic to be applied in combination with a pre-existing antibiotic(s). Indeed, attempts to define mechanisms of action often appear as the final step of plant investigations in the reviewed literature. Some of these antibacterial mechanisms may be broad spectrum in effect, meaning that the extract or compound(s) target cellular components or traits common to many bacteria. In other cases, effects may be narrower in range, demonstrable against only a small number of species, implying a mechanism of action targeting a relatively unique cellular component or trait. It is of course most desirable that a plant extract or compound will exert an antibacterial effect against a drug-resistant bacterial strain(s) through a novel mechanism of action, subverting drug-resistance capabilities. However, it is also possible that an extract or compound will act only through inhibition of the means by which a bacterial species is conferred drug-resistance, making it useful only as an adjunctive therapeutic.

Numerous bacterial resistance mechanisms to existing antibiotics and biocides have been identified and provide potential chemotherapeutic targets. These include drugefflux pumps, porin deletion, drug metabolism in the periplasmic space, alterations to membrane fluidity, the over-production or alteration of the drug target ${ }^{15}$, biofilms, and 
quorum sensing. Drug efflux pumps are energy-dependent, integral membrane proteins capable of expelling antibiotics across the cell membrane against their concentration gradient $^{91}$. The majority of such pumps in bacteria are non-specific proteins able to recognize and expel a broad range of structurally and chemically unrelated compounds ${ }^{6}$, and are believed to play an important role in bacterial pathogenesis, virulence and biofilm formation ${ }^{11}$. Porins, which are outer membrane proteins, are used by some small hydrophilic antibiotics as a means of entry to Gram negative bacteria ${ }^{91}$. Thus, deletion of these proteins presumably bars these antibiotics from entering the cell and exerting their effect(s). Alternatively, drug metabolism in the periplasmic space of Gram-negative bacteria is due to the presence of enzymes capable of breaking down foreign molecules introduced from outside of the cell ${ }^{61}$.

In contrast to porin deletion, alterations to cell membrane fluidity presumably bestow drug resistance by restricting the passage of hydrophobic antibiotics into the cell. It has been reported that in Gram-negative bacteria, the evolution of the outer membrane in combination with drug efflux pumps has come to provide a significant permeability barrier to amphipathic compounds as well ${ }^{11}$, and it is possible that alterations to the fluidity of the outer membrane fluidity play a role in this means of antibiotic resistance. Alternatively, over-production or alteration of the drug target presumably lessens or negates antibiotic effects by either increasing the drug concentration necessary for the exertion of these effects, or rendering the antibiotics ineffective through elimination of the binding site, respectively. Biofilm formation confers antibiotic resistance through the physical shielding of bacterial cells to drug exposure in an extracellular matrix. After initial attachment to host tissues for example, Escherichia coli begin to grow and spread 
on the surface as a monolayer, forming microcolonies which can eventually create biofilms ${ }^{65}$. Cells contained within these biofilms are protected from antibiotic exposure by the extracellular material, and have been reported to be as much as 1000-fold more resistant to antibiotics in comparison to planktonic cells ${ }^{91,92}$. Lastly, quorum sensing, or chemical communication among bacterial cells within a colony, potentially represents another drug-resistance mechanism, as it has been described as possibly regulating multidrug efflux pumps ${ }^{91}$. It has also been reported that the use of a quorum sensing inhibitor in combination with an antibiotic may increase bacterial biofilm susceptibility ${ }^{91}$.

While technically not drug-resistance mechanisms, other traits related to pathogenicity may provide additional opportunities for therapeutic interventions using plant extracts or compounds. For example, adhesion to eukaryotic cells often represents the first stage in many microbial infections, and bacterial traits such as cell surface hydrophobicity, or the specific binding of bacterial adhesins to the host cell-surface, are believed to play a role in these bacterium-host interactions ${ }^{43}$. Alternatively, some species, such as Pseudomonas aeruginosa for example, produce an extracellular shielding mechanism which confers resistance to the phagocytic activity of polymorphonuclear leukocytes $^{82}$. Therefore, as disruption of microbial adhesion or extracellular matrix formation by plant extracts or compounds may inhibit pathogenesis, these bacterial traits offer additional potential applications for plant extracts or compounds as therapeutics.

A limited number of assays measuring plant extract or compound effects on some of the antibiotic resistance mechanisms described above are found in the reviewed literature. Ohene-Agyei et al. used an in silico method to predict possible plant compound/drug efflux pump interactions, further confirming some of these predictions 
using an assay designed to assess reductions in the efflux of the dye Nile Red as a measure of pump inhibition by these plant compounds. Notably, they reported an increased sensitivity of drug-resistant bacterial strains to some synthetic antibiotics in the presence of these compounds ${ }^{11}$, suggesting that pump inhibition by the plant compounds prolonged the presence and activity of the synthetic antibiotics within the cells.

Alternatively, to test the plant compound dehydroleucodine's effects on biofilm formation, Mustafi et al. grew treated bacterial cells on polyvinyl chloride microtiter plates. The plates were stained with a crystal violet solution, washed, and the number of remaining attached cells quantified by solubilizing the dye in ethanol and measuring it using spectrophotometric means ${ }^{93}$. Tolmacheva et al., in the aforementioned study of Eastern-European medicinal plants, tested extract interference on quorum sensing abilities using the opportunistic plant pathogen Chromobacterium violaceum. Production of the purple pigment violacein is controlled by the same system as is responsible for quorum sensing activity in this organism, thus making possible a colorimetric assay, wherein reduction in pigment levels may be interpreted as indicative of antagonism of quorum sensing by the tested extract ${ }^{55}$.

Assays testing extract or compound effects on bacterial traits which do not provide a means of drug-resistance, but which still relate to pathogenicity, were more commonly found in the literature. Wojnicz et al. for example, in the previously described investigation of plants used to treat urinary tract infections, tested extract effects on the microbial adhesion and biofilm formation capabilities of a clinical strain of uropathogenic Escherichia coli. For example, the ability of extract-treated cells to aggregate in increasing salt concentrations was used to assess extract reductions in cell 
hydrophobicity, while hemagglutination of human erythrocytes was used as a means to assess extract impact on P. fimbriae expression or function, which is believed to play a role in microbial adhesion to host tissue. Additionally, the cell binding of congo red dye was used to assess extract effects on the expression of curli fibers, which may be involved in biofilm formation, while measurement of swimming zones was made to determine extract effects on motility, another virulence determinant in uropathogenic Escherichia coli strains ${ }^{65}$. Similarly, Voravuthikunchai and Limsuwan, in their aforementioned investigation of Thai medicinal plants, tested extract effects on the microbial adhesion of enterohemorrhagic strains of Escherichia coli, using an almost identical salt aggregation test, wherein the ability of bacteria to aggregate in increasing ammonium sulfate concentrations in the presence and absence of plant extract was assessed using light microscopy ${ }^{43}$.

Broad spectrum antibacterial mechanisms of action can be assessed as well. For example, effects on bacterial membrane permeability represent a potentially important mechanism of action, as even sub-lethal injuries to the membrane can affect the cell's ability to adequately osmoregulate or exclude toxic materials ${ }^{15}$, and these effects can be assessed using various assays. Lu et al. for example, tested the effects of formulated plant extracts on the cell membrane of the aquatic pathogen Aeromonas hydrophila through means of visual inspection via transmission electron microscopy, by measuring treated cells for potassium leakage with atomic absorption spectrometry, and using flow cytometry to detect propidium iodide nucleic acid intercalation ${ }^{88}$. Alternatively, Tomlinson and Palombo investigated Eremophila duttonii extract effects on Staphylococcus aureus membrane permeability using both detection of propidium iodide 
binding and a salt tolerance assay, which measured the ability of treated cells to grow on sodium-supplemented agar after extract exposure ${ }^{15}$.

Bacterial DNA damage by an extract or compound represents another broad spectrum mechanism of action, and can also be assayed. Stagos et al. for example, in investigating the potential protective effects of Greek Lamiaceae species extracts against hydroxyl radical-induced DNA strand damage, also tested the extracts themselves for potential DNA damaging effects. Gel electrophoresis of extract-treated pBluescript-SK plasmid DNA was used to detect conversion of supercoiled topological states to open and linear forms, which is indicative of DNA damage ${ }^{30}$. Also, Ganie et al. investigated potential DNA damage by a methanol extract of Arnebia benthamii, using a similar methodology with the plasmid pBR322 $2^{49}$.

Comparison of the antibacterial testing results of an extract or compound versus bacterial strains with different phenotypes can also yield clues to its general mechanism of action. For example, the detection of antibacterial activity against both Gram-positive and Gram-negative bacteria may indicate broad spectrum antibiotic compounds or general metabolic toxins ${ }^{23}$. Alternatively, reduced efficacy versus Gram-negative in comparison to Gram-positive bacteria may indicate a mechanism of action impeded by differences in cell wall composition ${ }^{8}$. In an investigation of the antibacterial efficacy of several Turkish plant extracts, Oskay et al. noted that the greater efficacy demonstrated by several of these extracts versus drug-resistant bacterial strains in comparison to the reference strains might be indicative of a unique mechanism of action ${ }^{8}$. However, comparisons may also yield clues as to the specific nature of a mechanism of action. For example, Ahmad et al. concluded that the increased inhibitory activities of Heydotis 
capitellata and Heydotis dichota extracts against a DNA-repair deficient Bacillus subtilis strain in comparison to the wild-type was suggestive of a possible DNA inhibitory mechanism of action ${ }^{94}$. Conversely, in other cases, such comparisons can also rule out a specific mechanism of action. For example, Yasanuka et al. concluded that the similar efficacies of Mexican medicinal plant extracts against both methicillin-sensitive and methicillin-resistant strains of Staphylococcus aureus suggested that lactam rings could be ruled out as a possible mechanism of action ${ }^{26}$.

Multiple possible mechanisms of action have been ascribed to multiple classes of plant compounds in the literature. Flavonoids for example, are believed to complex with both extracellular and soluble proteins, as well as detrimentally interact with enzymes essential for maintaining the stability of the cell wall ${ }^{22}$. There have also been reports of flavonoid inhibition of enzymes involved in mycolic and fatty acid biosynthesis 5 . Further, it has also been suggested that flavonoids may exert antibacterial effects or inhibit pathogenesis by inhibiting nucleic acid synthesis, cytoplasmic membrane function, microbial attachment and biofilm formation ${ }^{91}$. Biofilm inhibition, by flavonoids such as quercetin, kaempherol, apigenin and naringenin for example, has been attributed to suppression of autoinducer-2 activity, which plays a role in cell-to-cell communication $^{65}$. The toxicity of phenolics to bacterial cells has been ascribed to disruption of the cell membrane ${ }^{95}$, or possible non-specific protein interactions ${ }^{69}$. Catechins however, phenolic compounds found in green tea, have been reported to kill bacterial cells through specific inhibition of the enzyme DNA gyrase ${ }^{76}$. Polyphenolic compounds have been postulated to exert antibacterial effects not only through disruption of the cell membrane, but also by causing liposome leakage, by generating hydrogen 
peroxide, by exerting mutagenic effects, and/or by inhibiting or killing the cell through adsorbing onto the surface of the bacterial cell wall ${ }^{84,96}$. Saponins may affect cell membrane permeability through the formation of plasma membrane pores, and may also interfere with enzyme activity ${ }^{54}$. Tannins have been suggested to bind and form complexes with cellular enzymes, cell wall proteins and metal ions, and disrupt the cell membrane ${ }^{90}$. Terpenes have also been claimed to act through cell membrane disruption ${ }^{15}$.

General metabolic effects may be responsible for the antibacterial activity of some plant extracts and compounds. Oxidative stress, for example, has been reported to be involved in the lethality of antibiotics exerting their effects through different mechanisms of action ${ }^{97}$, and this may be the case with some plant extracts and compounds as well. It has been demonstrated that under antibiotic treatment, superoxide production results in the disruption of iron metabolism regulation, and the generation of highly toxic hydroxyl radicals ${ }^{97}$. Autocidal activities resulting from free radical accumulation due to metabolic imbalance and impaired ionic homeostasis may thus add to the biocidal effects of antibiotics or plant extracts and compounds ${ }^{15}$. For example, organic acids in plant extracts may act as antibacterial compounds when undissociated forms of the acids enter the cell and partially dissociate, thereby decreasing cytoplasmic $\mathrm{pH}$ and interfering with the proton motive force of the cell membrane ${ }^{72}$. Conclusion

There is a strong case to be made, based on several compelling arguments, for the increased exploration of plants, particularly "medicinal plants", as a potential source of novel antibiotic compounds with which to treat drug-resistant bacteria. Plants have a 
longstanding history as a source of therapeutics used in both traditional and modern medicine, and the sheer abundance of plant species which have yet to be investigated in this regard suggests that this natural resource may be thus far underutilized. Additionally, plants and plant products are still in wide use as traditional medicine by much of the world's population, thus there are benefits to be gained by assessing and improving the safety and standards of these products. Further, plant-derived antibiotics may offer the additional benefits, in comparison to synthetic antibiotics, of lessened toxicity and side effects, biodegradability, reduced cost and increased accessibility.

However, before they begin, the investigation of the antibacterial properties of plants can be complicated by logistical issues pertaining to the procurement of plant material for study. These issues can directly impact which plant(s), which plant part(s), and what amounts may be obtained. These impacts can affect experimental results, for example, due to potential fluctuations in the chemical composition of plants which may occur seasonally, or be dependent upon the geographic locations or microenvironments in which the plants were grown. In consideration of potential logistical issues, and given the abundance of available species from which to choose, reference to traditional medicinal practices may provide useful in the selection of specific plants or parts for study.

Additionally, the study of plants for the possession of novel antibiotic compounds is a field greatly lacking in the standardization of experimental methods. For example, even the treatment of plant material prior to chemical extraction varies greatly, and the effects such treatments may have on experimental results in unknown. Further, a wide variety of solvents and methods are used in the chemical extraction of plant material, and 
all of these factors combine to affect extract yields, and possibly experimental results. However, as the presence or chemical composition of an antibiotic compound in a plant is unknown prior to experimentation, trial and error methods may be unavoidable in thorough investigations. However, reproduction of traditional preparation and extraction methods can help refute or support historical claims of a plant's medicinal benefit.

This field of research is further hindered by the variety of assays used in the antibacterial testing of plant extracts. The use of solid media assays versus microbroth dilution methods, as well as discrepancies in bacterial inoculum levels and working concentrations of extracts tested, makes study comparisons and recognition of potentially significant results difficult. Further, studies often neglect to include assays for extract toxicity to eukaryotic cells or organisms. Definitive identification of the compound(s) within a plant extract which are responsible for its antibacterial activity appears only sporadically in the literature, though this may be due in some cases to lack of access to more advanced instrumental methods. Finally, testing of the mechanism of action by which the antibacterial effects of a plant extract or compound are exerted, are few in number as well. Perhaps as a result, several plant compound classes are ascribed in the literature to exert antibacterial effects by a multitude of different mechanisms.

However, despite the difficulties inherent to these investigations, there is a preponderance of encouraging results to be found in these written works. Crude extracts and compounds derived from plant material have demonstrated antibacterial activity against a wide array of disease-relevant bacterial species. Importantly, they have also demonstrated an ability to enhance the efficacy of standard antibiotics when combined together in the treatment of these bacteria. Given the number and chemical diversity of 
plant compounds so far identified as possessing antibacterial properties, it is unreasonable to expect that a single chemical extraction procedure can be universally applied to all plant species, though adherence to historical practices may at least be useful in validating the efficacy of traditional medicines. Still, in the future, standardization of the antibacterial testing methods used in this field, along with an increased emphasis on assaying drug-resistant bacterial pathogens, may help better identify which extracts and compounds are effective in concentrations low enough to be considered of therapeutic benefit, while standardization of toxicity assays may better ensure their safety. Nevertheless, the results appearing in the reviewed literature have further verified the existence of antibacterial compounds in a variety of plant species, and when it is considered that the overwhelming majority of species have yet to be investigated in such a manner, the continuation and expansion of this work appears to hold great promise. 


\section{MATERIALS, METHODS AND RESULTS}

\section{Artemisia}

The genus Artemisia consists of some 522 species $^{98}$ of annual or perennial shrubby or herbaceous plants ${ }^{99}$, belonging to the Anthemideae tribe of the Asteraceae or "Compositae" family ${ }^{100}$. The genus is globally distributed amongst a variety of habitats, being found in northern temperate regions of Europe and North America, as well as in South America, southern Africa and the Pacific Islands ${ }^{98,100,101}$. These plants possess an $\operatorname{aromatic~odor~}^{99}$, believed to be attributable to the presence of volatile terpenes ${ }^{100}$. However, the scent is similar to that of various salvia species, a genus of the Lamiaceae or "mint" family, and it has been suggested that it is this relationship which explains the "sage" portion of the common nomenclature for several Artemisia species, such as "sagebrush", "sagewort" or "sageweed"102.

Artemisia tridentata, in addition to being the most common shrub species found in the American West, is one of the most ecologically important and widely distributed shrub species in western North America, growing in dry mountain basins and high deserts ranging from British Columbia to Baja, and extending east through the Rocky Mountains and the Dakotas ${ }^{100,102}$. It is the largest of the shrubs in this genus, growing as large as four feet, and serves both as an important habitat and food source for animals and invertebrates ${ }^{100,102}$. The plant has a silvery gray appearance, with alternate leaves divided into three parts at their tips ${ }^{102}$. The blooming period runs from July through September, with the flowers small, tubular, yellowish, and growing in loosely arranged terminate inflorescences $^{102}$. 
Artemisia species in general, and the tridentata species specifically, have extensive histories of use in traditional medicine. Plants of this species are believed to possess bacteriostatic qualities capable of combatting various forms of infection, and have been used for such purposes in topical applications ${ }^{102}$. Though they have also been used for internal applications, such as in the treatment of internal bleeding, such practices are discouraged by modern-day herbalists, due to potential liver and digestive tract toxicity ${ }^{102}$. Historically, herbs from this genus have also been used, for example in Anatolian medicine, as tonics, for antimalarial and antihelmintic purposes, for diabetes, bronchitis, ulcers, tuberculosis, and in wound treatments ${ }^{98}$. Artemisia species have also been reported to have been used to treat fever, malaria, tuberculosis and intestinal worms ${ }^{101}$. Even today, these species are one of the most popular in Chinese traditional preparations, used for the treatment of malaria, hepatitis, inflammation and infections resulting from fungi, bacteria and viruses, as well as in cancer treatment ${ }^{100}$. Further, the essential oils of these species are used in a variety of antimicrobial applications, including embalmment, food preservation, as microbicidals, as well as in applications as sedative, analgesic, spasmolytic, anti-inflammatory and local anesthetic remedies, with 300 commercially important varieties important to the pharmaceutical, cosmetic, perfume, food, agricultural, and sanitary industries ${ }^{100}$.

Artemisia tridentata is also seen in a variety of medicinal applications in modern times. Mexican Indians in Colorado for example, use it in the form of tea or hot vapor baths to treat colds ${ }^{103}$. Alternatively, New Mexico Spanish-Americans apply it in an external poultice to the umbilical stump in order to prevent infection, and to the small of the back as both an anti-infective remedy following miscarriage, and as a treatment for 
menstrual cramps ${ }^{103}$. The Navajo use this plant for a variety of medical purposes: employing its odor to treat headaches, using boiled plant material as a childbirth aid or for treatment of indigestion and constipation, for making a poultice used in the treatment of colds, swellings, tuberculosis and corns, or to make tea for the treatment of colds and fevers $^{103}$. The Hopi, Tewa and Zuni are also reported to use Artemisia species, including tridentata, for medicinal purposes ${ }^{103}$.

Bacterial Species Assayed

Staphylococcus aureus is a member of the genus Staphylococcus, comprised of at least 45 species of gram-positive, non-motile, spherical bacteria, approximately $1 \mu \mathrm{m}$ in diameter, which often grow in irregular, grapelike clusters ${ }^{104}$. They comprise some members of the natural microbiota of human skin and mucous membranes ${ }^{104}$. In fact, it has been estimated that approximately $30 \%$ of all people are rhinal carriers of Staphylococcus aureus ${ }^{105}$. Staphylococcus infection typically occurs through the formation of a furuncle, by means of contamination of a wound, through contamination of implanted medical devices, or via urinary tract infections ${ }^{104}$. Infections resulting from some staphylococci may cause suppuration, abscess formation, pyogenic infections, or potentially fatal septicemia, while pathogenic species produce extracellular enzymes and toxins, and infections with these species may lead to blood hemolysis or plasma coagulation $^{104}$. It is noteworthy that the most common type of food poisoning results from ingestion of a staphylococcal heat-stable enterotoxin ${ }^{104}$.

Staphylococcus aureus is the major human pathogen of this genus, and it is believed that most people will incur a Staphylococcus aureus infection at some point in their lifetimes, ranging from food poisoning and mild skin infections to more serious, 
potentially fatal conditions, such as pneumonia, osteomyelitis, endocarditis, bacteremia and sepsis ${ }^{104,105}$. Staphylococcus aureus and Staphylococci in general have become resistant to multiple antibiotics, due in part to the acquisition or development of the "Staphylococcal cassette chromosome," a genetic element encoding a penicillin-binding protein enabling resistance to methicillin, nafcillin and oxacillin, though this element may contain genes conferring resistance to additional antibiotics as well ${ }^{104} \cdot \beta$-lactamase production is also observed in staphylococci, exclusive of the cassette chromosome, and imparting penicillin resistance ${ }^{104}$. Vancomycin resistance is seen in this genus as well, resulting from alterations and proportional changes in cell wall components, while resistance to erythromycins, tetracyclines, aminoglycosides and other drugs is the result of plasmid-acquired resistance ${ }^{104}$. Further, as mentioned above, staphylococci produce various enzymes and toxins important in their pathology. In addition to enzymes such as clumping factor and coagulase, Staphylococcus aureus produces hemolysins, the toxin Panton-Valentine Leukocidin, exfoliative toxins, toxic shock syndrome toxin-1, and other enterotoxins $^{104}$

In a 2014 World Health Organization (WHO) global surveillance study of antimicrobial resistance, the prevalence of infections due to methicillin-resistant Staphylococcus aureus (MRSA) strains among all Staphylococci infections was assessed. More than 25 percent of Staphylococci infections were attributed to MRSA in Southeast Asia, greater than 50 percent in some Eastern Mediterranean regions, greater than or equal to 60 percent in some parts of Europe, approximately 80 percent in some African regions, greater than or equal to 80 percent in the Western Pacific, and as high as 90 percent in some regions of the Americas. However, the data was admittedly incomplete 
due to the difficulties of tracking these infections in countries without the policies and procedures in place to collect such information ${ }^{106}$. Therefore, it is possible that the WHO estimates may in fact underestimate the prevalence of MRSA. Finally, it was determined that those infected with a MRSA strain were $64 \%$ more likely to succumb to infections than were those infected with non-resistant strains, providing some metric of the potential mortality attributable to this drug-resistant species ${ }^{106}$.

Pseudomonas aeruginosa is a member of the family Pseudomonadaceae, which consists of gram-negative, motile, aerobic rods, occurring widely in soil, water, plants and animals ${ }^{104}$. Pseudomonas aeruginosa is the major human pathogen of the genus Pseudomonas, normally found in moist environments, in or on the human intestine or skin, and in hospitals ${ }^{104}$. Infection is typically the result of bacterial circumvention of host defenses by means of a physically compromised site providing a route of entry, such as a burn, puncture wound or catheter for example ${ }^{104}$, with most infections nosocomial in nature and primarily affecting the immunocompromised, potentially resulting in infections of the blood, pneumonia, or post-surgery complications ${ }^{107}$. More serious infections may manifest as fever, shock or oliguria, among other conditions, while milder manifestations include ear infections in children or skin rashes, both believed to be attributable to insufficiently chlorinated water in pools or hot tubs ${ }^{104,107}$.

Pseudomonas aeruginosa possesses fimbriae for host-cell attachment, and produces exopolysaccharide and sometimes lipopolysaccharide, the latter of which imparts some endotoxic effects and plays a direct role in the more serious infections described above ${ }^{104}$. Also, most clinical isolates produce extracellular elastases, proteases and hemolysins ${ }^{104}$. Further, Pseudomonas aeruginosa strains may produce the 
necrotizing Exotoxin A, as well as exoenzymes S, T, U and Y, which are also toxins and are believed to cause cell death or adversely affect the host immune response ${ }^{104}$. Centers for Disease Control data for Pseudomonas aeruginosa infections in the United States include 51,000 healthcare-associated annual incidences, 6,000 of which are attributable to multi-drug resistant strains, with 400 annual deaths ${ }^{107}$.

Listeria monocytogenes is a member of the Listeria genus, and is a gram-positive, non-spore forming, facultative anaerobic motile $\operatorname{rod}^{104}$. There exist 13 known serovars, with clinical isolates typically $0.4-0.5 \mu \mathrm{m}$ in diameter and 0.5-2 $\mu \mathrm{m}$ in length ${ }^{104}$. Commonly found in soil, water and animals (CDC), this species is extremely resilient, capable of tolerating acidic and high salt environments, as well as refrigeration temperatures of $4{ }^{\circ} \mathrm{C}^{104}$. Listeriosis is the result of the ingestion of contaminated food or food products, such as uncooked or undercooked meats, vegetables or dairy products, though contamination may also occur during food processing at a manufacturing facility ${ }^{108}$. Those most susceptible to infection or disease are newborns, the pregnant, the elderly, or the immunocompromised, with in utero transmission possible ${ }^{108}$. Clinical manifestations in the immunocompromised include gastroenteritis, bacteremia, meningoencephalitis and septicemia, as well as neonatal sepsis and meningitis, and postpartum infections ${ }^{104}$. Listeria monocytogenes is also known to cause disease in animals, both domestic and wild ${ }^{104}$.

Listeria monocytogenes produces several adhesion proteins for the purposes of host-cell attachment, as well as internalins on its cell wall which promote phagocytosis by epithelial cells ${ }^{104}$. The in vivo life cycle of this species transpires intracellularly among epithelial cells, macrophages and hepatocytes, allowing it to avoid exposure to 
antibodies, complement or polymorphonuclear leukocytes ${ }^{104}$. This complicates antibiotic therapy, as the drugs must enter the eukaryotic host cells to exert their effects ${ }^{104}$. According to the Centers for Disease Control, from 2000-2008, Listeria monocytogenes infections resulted in 1,600 illnesses each year in the United States, with 1,500 resultant hospitalizations and 250 annual deaths ${ }^{108}$.

Salmonellae are members of the Enterobacteriaceae family, a grouping composed of gram-negative, mostly motile, flagellous, rod-shaped facultative anaerobes or aerobes ${ }^{104}$. These are among the most common disease-causing bacteria, with 20-25 clinically relevant species ${ }^{104}$. Most are animal pathogens, and the animals they typically infect, such as pigs, poultry, rodents, pets and others, act as bacterial reservoirs. Salmonella enterica is one of two species of the Salmonella genus, which consists of greater than 2500 serotypes $^{104}$. It can be further divided into at least five subspecies, with enterica representing the subspecies of greatest clinical relevance to humans ${ }^{104}$.

Salmonellae are also fairly resilient, for example, being capable of surviving in freezing water for extended periods. Human infection or disease is the result of the ingestion of contaminated foods, such as meat products, shellfish, dried or frozen eggs, beverages such as water or milk and dairy products, recreational drugs such as marijuana, animal dyes and household pets ${ }^{104}$. The most at-risk populations again are infants, the elderly, and the immunocompromised ${ }^{109}$. Clinical manifestations include bacteremia, enteric fever, enterocolitis and possible systemic infection ${ }^{109}$. According to the Centers for Disease Control, from 2000-2008, on an annual basis the United States witnessed 1 million foodborne illnesses, 19,000 hospitalizations and 380 deaths resulting from non- 
typhoidal Salmonella species, with an additional 1,800 annual illnesses and 200 annual hospitalizations due to Salmonella enterica serotype Typhi ${ }^{109}$. 


\section{Preliminary Plant Screenings}

Fifteen plants, from five families, were screened for potential antibacterial properties prior to the investigation of Artemisia tridentata. The extracts tested were preexisting in the lab, having been created from the boiling of dried plant material in methanol, cotton filtration of the supernatant, solvent removal via rotary evaporation, desiccation, and suspension of the dried extract in dimethyl sulfoxide (DMSO) after solvent removal. The species and family names of the plants from which each extract was derived, the concentration assayed, the number of samples run in the assay, and the incubation conditions are summarized in Table 1 below and appear in chronological order.

\begin{tabular}{|c|c|c|c|c|}
\hline Species Name & Family & $\begin{array}{c}\text { Tested Extract } \\
\text { Concentration }\end{array}$ & Sample Repeats & Assay Conditions \\
\hline Garciadelia castilloae & Euphorbiaceae & $100 \mu \mathrm{g} / \mathrm{ml}$ & Single & $39^{\circ} \mathrm{C}$ and $235 \mathrm{rpm}$ \\
\hline Grimmeodendron eglandulosum & Euphorbiaceae & $100 \mu \mathrm{g} / \mathrm{ml}$ & Single & $37^{\circ} \mathrm{C}$ and $235 \mathrm{rpm}$ \\
\hline Lasiocroton bahamensis & Euphorbiaceae & $100 \mu \mathrm{g} / \mathrm{ml}$ & Triplicate & $37^{\circ} \mathrm{C}$ and $235 \mathrm{rpm}$ \\
\hline Omphelia ekmanii & Euphorbiaceae & $100 \mu \mathrm{g} / \mathrm{ml}$ & Triplicate & $37^{\circ} \mathrm{C}$ and $235 \mathrm{rpm}$ \\
\hline Phyllanthus epiphyllanthus & Phyllanthaceae & $100 \mu \mathrm{g} / \mathrm{ml}$ & Triplicate & $37^{\circ} \mathrm{C}$ and $235 \mathrm{rpm}$ \\
\hline Theophrasta jussieui & Primulaceae & $100 \mu \mathrm{g} / \mathrm{ml}$ & Triplicate & $37^{\circ} \mathrm{C}$ and $250 \mathrm{rpm}$ \\
\hline Catesbaea parviflora & Rubiaceae & $100 \mu \mathrm{g} / \mathrm{ml}$ & Triplicate & $37^{\circ} \mathrm{C}$ and $250 \mathrm{rpm}$ \\
\hline Cubanola daphnoides & Rubiaceae & $200 \mu \mathrm{g} / \mathrm{ml}$ & Triplicate & $37^{\circ} \mathrm{C}$ and $250 \mathrm{rpm}$ \\
\hline Cubanola domingensis & Rubiaceae & $200 \mu \mathrm{g} / \mathrm{ml}$ & Triplicate & $37^{\circ} \mathrm{C}$ and $250 \mathrm{rpm}$ \\
\hline Isadorea pungens & Rubiaceae & $200 \mu \mathrm{g} / \mathrm{ml}$ & Triplicate & $37^{\circ} \mathrm{C}$ and $250 \mathrm{rpm}$ \\
\hline Osa pulchra & Rubiaceae & $200 \mu \mathrm{g} / \mathrm{ml}$ & Triplicate & $37^{\circ} \mathrm{C}$ and $250 \mathrm{rpm}$ \\
\hline Pilea grandifolia & Urticaceae & $200 \mu \mathrm{g} / \mathrm{ml}$ & Triplicate & $37^{\circ} \mathrm{C}$ and $250 \mathrm{rpm}$ \\
\hline Phyllanthus myriophyllus & Phyllanthaceae & $600 \mu \mathrm{g} / \mathrm{ml}$ & Duplicate & $37^{\circ} \mathrm{C}$ and $250 \mathrm{rpm}$ \\
\hline Clavija domingensis & Primulaceae & $600 \mu \mathrm{g} / \mathrm{ml}$ & Duplicate & $37^{\circ} \mathrm{C}$ and $250 \mathrm{rpm}$ \\
\hline Pilea microphylla & Urticaceae & $600 \mu \mathrm{g} / \mathrm{ml}$ & Duplicate & $37^{\circ} \mathrm{C}$ and $250 \mathrm{rpm}$ \\
\hline
\end{tabular}

Table 1: Summary of preliminary plant screening assays

All extracts were assayed against the following four species of bacteria:

\section{Staphylococcus aureus, Pseudomonas aeruginosa, Listeria monocytogenes, and}

Salmonella enterica. Bacterial cultures were inoculated from frozen stock into Luria-

Broth (LB) and incubated with rotation overnight. The following day, the cultures were

diluted in $5 \mathrm{ml}$ fresh LB to an approximate optical density reading of 0.100 , measured at 
a wavelength of $600 \mathrm{~nm}\left(\mathrm{OD}_{600}\right)$. Measurements were read using an Ultrospec 2100 Pro UV/Visible Spectrophotometer (Amersham Biosciences), and were taken again after the addition of sample treatments, and at 1,2, 3, 4 and 24-hour time points post-incubation. Initial values, or the averages of values for samples run in duplicate or triplicate, taken after sample treatment but prior to incubation, were subtracted from all subsequent time point readings to account for the light dispersal contributions of the media, the antibiotics, and the extracts, the last of which were quite significant in many cases. Negative values were recorded as a reading of zero.

Untreated bacterial cultures served as negative controls. Initially, the positive controls for each bacterial species were as follows and were based on previously experimentally determined values (data not shown): tetracycline at a concentration of 25 $\mu \mathrm{g} / \mathrm{ml}$ for Staphylococcus aureus, and amikacin at a concentration of $100 \mu \mathrm{g} / \mathrm{ml}$ for Pseudomonas aeruginosa, Listeria monocytogenes and Salmonella enterica. However, after the first assay, the concentration of amikacin used as a positive control for Listeria monocytogenes was increased to $200 \mu \mathrm{g} / \mathrm{ml}$, as the lesser concentration failed to satisfactorily inhibit bacterial growth for a full 24 hours.

Under the specific conditions used in these assays, none of the methanol extracts demonstrated any discernable antibacterial effects against the bacterial species tested (data not shown). This was despite increases in the extract concentrations assayed, as can be seen in Table 1. Additionally, the growth curves observed for both the treated and untreated samples often dipped between the 3 and 4-hour time points, implying overgrowth of the culture in the volume of media used, likely attributable to an excessive initial inoculum level. 


\section{Initial Artemisia tridentata Assays}

Artemisia tridentata leaves and stems were collected in November of 2012 in Ephrata, Washington, and their identity verified by botanist Dr. David W. Lee. The plant material was placed inside a heated cabinet without light at approximately $80^{\circ} \mathrm{F}$ until dry. The first extract was created from this material by boiling $4.9758 \mathrm{~g}$ of mostly leaves in 50 $\mathrm{ml}$ methanol (approximate $1 \mathrm{~g} / 10 \mathrm{ml}$ plant material to solvent ratio) with the use of a reflux condenser. The material was boiled for approximately 45 minutes, and the resulting supernatant decanted and filtered through cotton. $50 \mathrm{ml}$ of fresh methanol was added to the plant material, and the process was repeated. The combined extracts were dried using a rotary evaporator, then placed in a desiccator under vacuum to remove any remaining methanol. The final extract was then suspended in DMSO to a concentration of $100 \mu \mathrm{g} / \mathrm{ml}$.

This extract was tested for antibacterial properties in triplicate at a concentration of $100 \mu \mathrm{g} / \mathrm{ml}$, employing the same experimental conditions as were used in the screening of the preceding fifteen plant extracts. Bacterial cultures were grown overnight at approximately $37^{\circ} \mathrm{C}$ and $250 \mathrm{rpm}$ for a period of about 16 hours and 30 minutes. After dilution and treatment, samples were again incubated at approximately $37^{\circ} \mathrm{C}$ and 250 rpm. The Artemisia tridentata extract had no apparent growth inhibitory effects (data not shown). Also, there was again evidence of bacterial overgrowth.

These results came as a surprise, as an essential oil derived from this plant had been previously reported to inhibit the growth of Staphylococcus aureus ${ }^{99}$. Therefore, the assay was repeated as before, running single extract-treated samples at concentrations of 100,500 , and $1000 \mu \mathrm{g} / \mathrm{ml}$. Bacterial cultures were grown overnight at approximately 
$37^{\circ} \mathrm{C}$ and $250 \mathrm{rpm}$ for a period of about 17 hours and 15 minutes. After culture dilution and treatment, samples were again incubated at approximately $37^{\circ} \mathrm{C}$ and $250 \mathrm{rpm}$. As can be seen in figures 1-4, this extract showed some growth inhibition of Staphylococcus aureus at the highest concentration of $1000 \mu \mathrm{g} / \mathrm{ml}$, but only to a modest extent, and this effect dissipated after 4 hours, disappearing by the 23-hour mark (time constraints forced the last measurement to be taken one hour early).

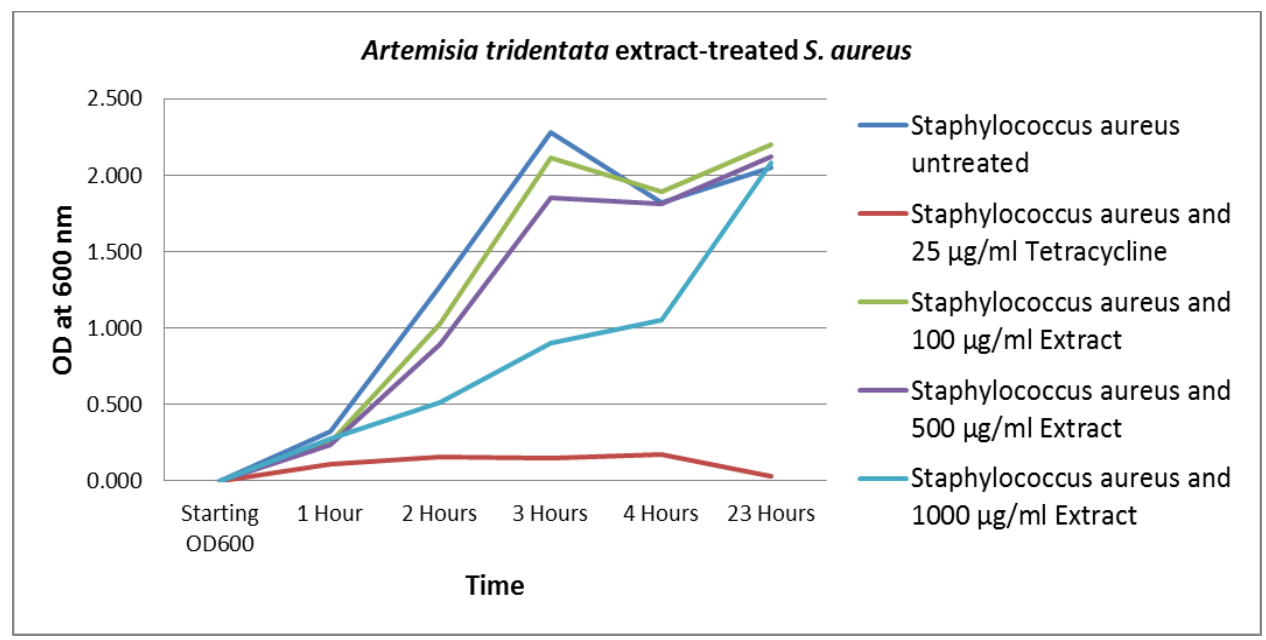

Figure 1: S. aureus treated with A. tridentata methanol extract

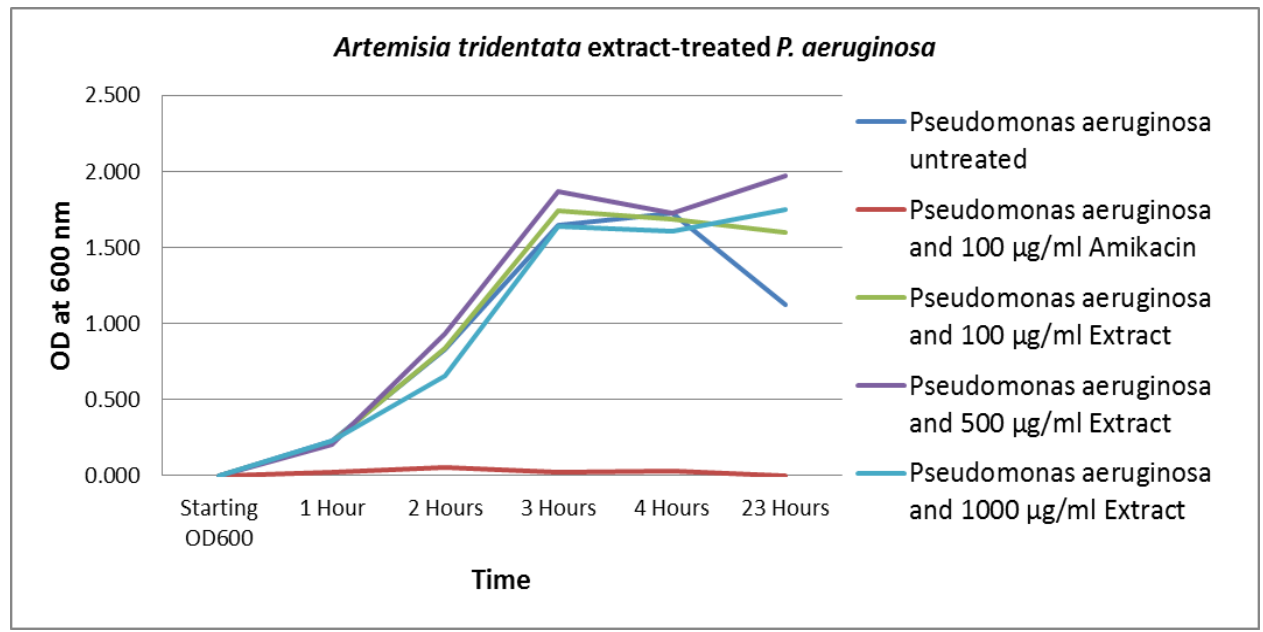

Figure 2: $P$. aeruginosa treated with $A$. tridentata methanol extract 


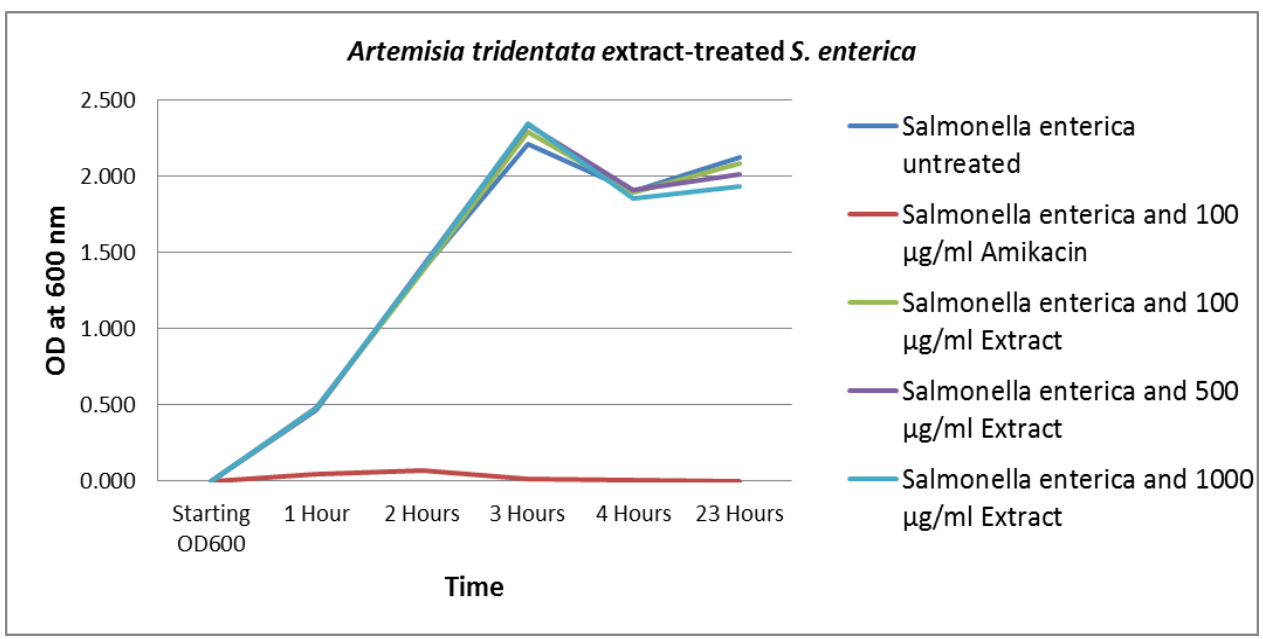

Figure 3: S. enterica treated with A. tridentata methanol extract

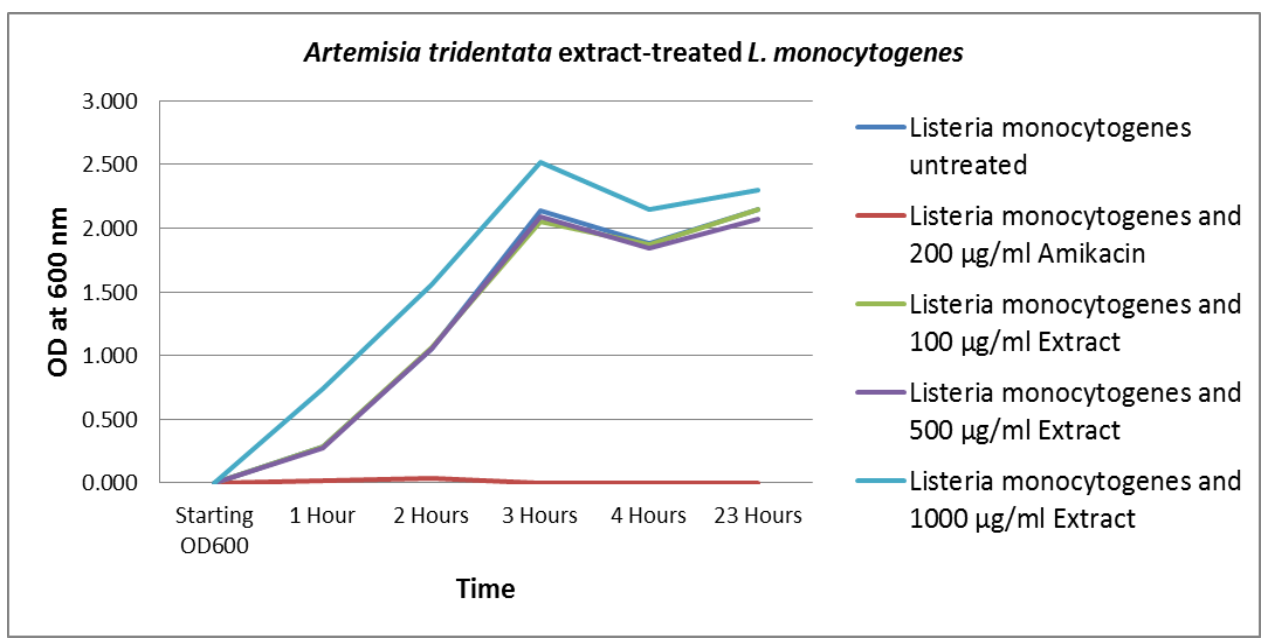

Figure 4: L. monocytogenes treated with A. tridentata methanol extract

A second methanol extract was made from the same Artemisia tridentata plant material for further testing. $2.0018 \mathrm{~g}$ of previously dried plant material, including leaves, some buds and stems, was boiled in $20 \mathrm{ml}$ methanol (approximate $1 \mathrm{~g} / 10 \mathrm{ml}$ plant material to solvent ratio) with the use of a reflux condenser. This material was boiled for approximately 45 minutes, and the resulting supernatant decanted and filtered, this time using Whatman \#1 filter paper in an effort to reduce the amount of insoluble material in 
the final extract. $20 \mathrm{ml}$ of fresh methanol was added to the plant material, and the process was repeated. The combined extracts were dried using a rotary evaporator, then placed in a desiccator under vacuum to remove any remaining methanol. The final extract was then suspended in DMSO to a concentration of $100 \mu \mathrm{g} / \mathrm{ml}$.

The antibacterial assay was repeated using this new extract, running extracttreated samples in triplicate, but only at a concentration of $1000 \mu \mathrm{g} / \mathrm{ml}$. Bacterial cultures were grown overnight at approximately $37^{\circ} \mathrm{C}$ and $225 \mathrm{rpm}$, for a period of about 19 hours and 20 minutes. After culture dilution and treatment, samples were again incubated at approximately $37^{\circ} \mathrm{C}$ and $225 \mathrm{rpm}$. A DMSO-treated sample, using a volume equivalent to the volume of extract added, was included as a vehicle control for each bacterial species. As can be seen in figure 5 (results for other bacteria not shown), this extract exhibited only slight inhibition of Staphylococcus aureus, and tetracycline as a positive control failed to inhibit growth for a full 24 hours.

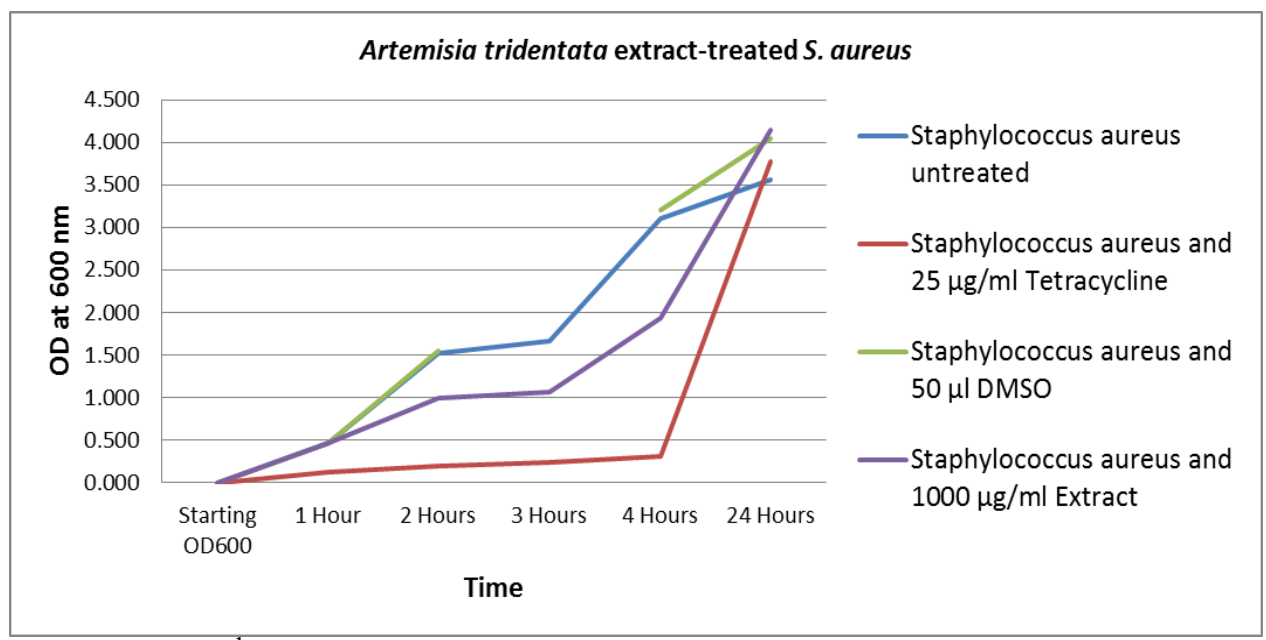

Figure 5: $2^{\text {nd }}$ trial of $S$. aureus treated with A. tridentata methanol extract 
Upon further review of the aforementioned paper, "Antibacterial Action of Essential Oils of Artemisia as an Ecological Factor," from 1967, it was observed that the bacterial inoculum level used in that study was significantly lower than that used in the assays described above ${ }^{99}$. The authors reported diluting bacterial cultures to levels of 1 $\mathrm{x} 10^{4}$ to $10^{5}$ bacteria per ml prior to sample treatment. However, they also reported essential oil treatments in terms of volumes added, with no concentrations given, thus direct comparisons to our treatments was difficult. The number of bacteria giving an $\mathrm{OD}^{600}$ reading of 1.000 is believed to be approximately $8 \times 10^{8}$ cells $/ \mathrm{ml}^{110}$. However, this number is representative of an Escherichia coli culture, and as Staphylococcus aureus possesses a different size and cell morphology, it likely disperses the light passed through a culture sample to a different extent, making the use of this number only an estimate.

Therefore, the assay was repeated yet again to assess whether the mild inhibitory effect observed for Artemisia tridentata versus Staphylococcus aureus in the previous assays was due to the use of too high a bacterial inoculum level. Extract-treated samples were tested in duplicate at a concentration of $1000 \mu \mathrm{g} / \mathrm{ml}$. Bacterial cultures were grown overnight at approximately $37^{\circ} \mathrm{C}$ and $225 \mathrm{rpm}$ for a period of about 20 hours and 15 minutes. However, this time, overnight cultures were diluted to an $\mathrm{OD}_{600}$ value of approximately 1.000 , following which $2 \mu \mathrm{l}$ of these diluted cultures were used to inoculate $5 \mathrm{ml}$ samples of fresh LB. Based on the Escherichia coli optical density number, this should have resulted in a concentration of approximately 320,000 bacteria/ml $\left(\left[8 \times 10^{8}\right.\right.$ cells $\left.\left./ \mathrm{ml} \times 0.002 \mathrm{ml}\right] / 5 \mathrm{ml}\right)$. This is significantly less than the $8 \times 10^{7}$ bacteria/ml used in the previous assays (based on the dilution of culture samples in those assays to an $\mathrm{OD}_{600}$ value of 0.100 , the equivalent of a 10 -fold dilution of a culture giving 
an $\mathrm{OD}_{600}$ reading of 1.000). After treatment and inoculation, samples were again incubated at approximately $37^{\circ} \mathrm{C}$ and $225 \mathrm{rpm}$. DMSO-treated samples were included as vehicle controls. $\mathrm{OD}_{600}$ readings were taken prior to incubation, and at 1, 2, 3, 4, 6 and 24-hour time points.

As can be seen in figure 6, overall growth of the Staphylococcus aureus cultures was slow, which would be expected given the low inoculum level. However, the extract prevented detectable growth of Staphylococcus aureus through the 6-hour time point. Though this effect disappeared by the 24-hour mark, it was the first demonstration of efficient growth inhibition using this extract/bacterial combination. There was no visible inhibition of the other bacterial species, with the exception of Pseudomonas aeruginosa, which appeared to demonstrate some minor extract effects. However, this effect failed to appear when investigated in additional trials (data not shown).

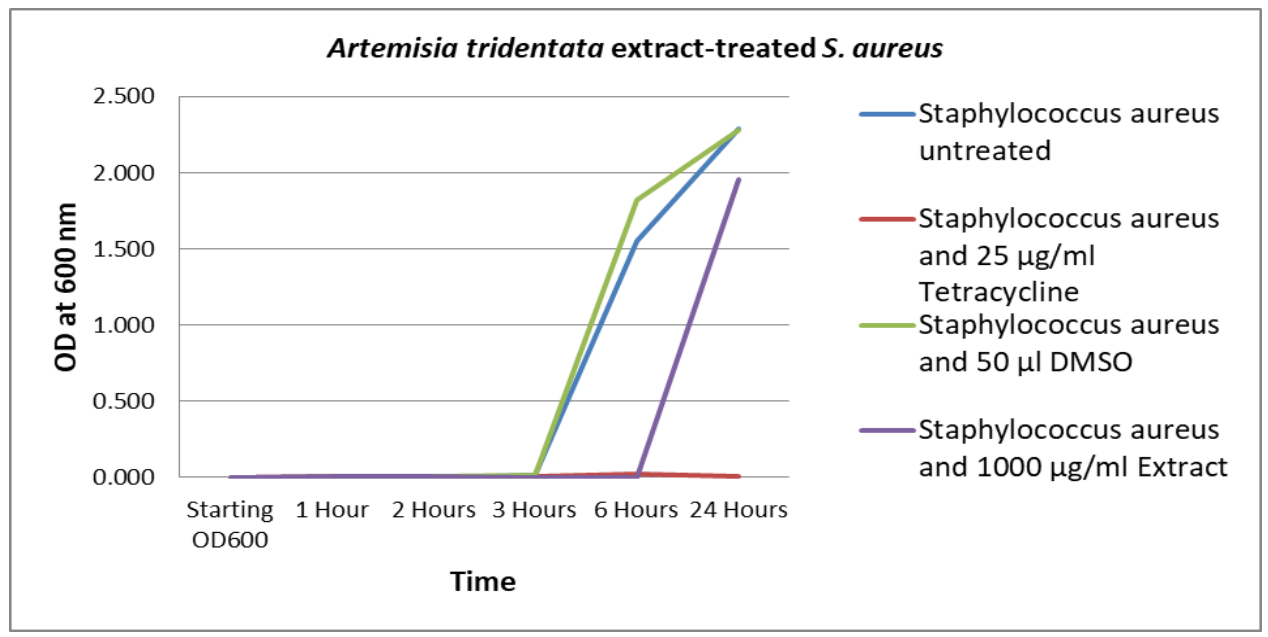

Figure 6: $3^{\text {rd }}$ trial of $S$. aureus treated with A. tridentata methanol extract 
Growth Inhibition and Combination with Antibiotics

Based upon these positive results, demonstrating Artemisia tridentata methanol extract growth inhibition of Staphylococcus aureus, additional methanol extracts were created from the same plant material. Eventually, these were fractionated into hexane, ethyl acetate and water extracts, as will be described below, and the antibacterial efficacies of each assayed. None showed growth inhibitory effects versus the other bacterial species investigated, acting only on Staphylococcus aureus (data not shown), with each extract demonstrating some efficacy against this species. However, it was noted that there was some variation in the antibacterial assay results among the various methanol extracts, despite their having been originally derived from the same original plant material. This discrepancy was likely attributable to variations in extraction yield. Therefore, for the sake of producing a set of consistent results for evaluation, the antibacterial trials described in the following pages were performed with an extract derived from a single sample of plant material.

43.6105 g of Artemisia tridentata dried plant leaves (buds and stems were excluded as much as possible) was ground with a mortar and pestle, and subjected to boiling in methanol with the use of a reflux condenser. Approximately $258 \mathrm{ml}$ of methanol was added to the plant material ( $\approx 6 \mathrm{ml}$ solvent per $\mathrm{g}$ of plant material), and the mixture boiled for about 45 minutes. The supernatant was decanted, an approximate equivalent volume of fresh methanol was added to the plant material, and the boiling process was repeated. Each supernatant was independently filtered through cotton after boiling, and the pooled filtrates then passed through Whatman \#1 filter paper. 
The resultant solution had a remaining volume of nearly $350 \mathrm{ml}$, with some methanol likely absorbed by the plant material or lost during the boiling process despite the use of a reflux condenser. Using separatory funnels, this solution was fractionated into hexane using an approximately equivalent volume, with an additional $50 \mathrm{ml}$ hexane added during the process to enhance visible separation of the solvents. The hexane portion was filtered through Whatman \#1 filter paper to remove visible particulate matter, dried using rotary evaporation, and desiccated under vacuum. The methanol portion was also dried using these methods.

The dried methanol extract was eventually re-suspended in ethyl acetate, and this solution fractionated with deionized water. Approximate volumes of $300 \mathrm{ml}$ of ethyl acetate and $240 \mathrm{ml}$ of water were necessary to achieve visible separation of the solvents and avoid saturation of either. Interestingly, overnight storage of the fractions at $4^{\circ} \mathrm{C}$ resulted in further separation of ethyl acetate in the water portion, suggesting that the fractionation of these two solvents might be enhanced in the future through the use of longer wait times. In addition to the top layer of immiscible ethyl acetate, there was also a middle layer visible between this and the aqueous layer, appearing to consist of fat micelles. Both of these top two layers were removed, and the remaining water portion filtered through Whatman \#1 filter paper. The original ethyl acetate portion also had some visible fat micelles, though an attempt to remove these by means of paper filtration was unsuccessful. This ethyl acetate mixture was dried in a manner similar to the methanol and hexane solutions, while the water portion required freeze drying to remove the solvent. All dried extracts were suspended in DMSO to a concentration of $100 \mu \mathrm{g} / \mathrm{ml}$. 
The antibacterial assays testing these extracts were performed in 96-well plates. Prior to inoculation, all wells contained a final volume of $100 \mu l$. Overnight cultures of Staphylococcus aureus were routinely grown at approximately $37^{\circ} \mathrm{C}$ and $225 \mathrm{rpm}$ for a length of 16-17 hours, as it was observed from the results of earlier experiments that harvesting the cultures during this time frame resulted in better growth of untreated bacterial controls in the assays, thereby enhancing detection of antibacterial effects. The following day, the cultures were diluted to an $\mathrm{OD}_{600}$ reading between $0.950-1.050$, and 2 $\mu \mathrm{l}$ culture added to the appropriate wells. Again using the Escherichia coli optical density standard for numeration, this should have resulted in approximate cell counts of $1.52-1.68 \times 10^{7}$ cells $/ \mathrm{ml}\left(\left[8 \times 10^{8} \mathrm{cell} / \mathrm{ml} \times 0.002\right] / 0.1 \mathrm{ml}\right)$, less than the inoculum level used in the screenings of the other plant species described above, but still considerably greater than those used in the aforementioned 1967 study.

Untreated LB samples served as sterility controls, untreated Staphylococcus aureus samples were used as negative controls, and samples treated with $25 \mu \mathrm{g} / \mathrm{ml}$ tetracycline served as positive controls. DMSO vehicle controls were no longer included due to a lack of available space on the plates, and the fact that the highest concentration of extract assayed required the addition of a lower volume than that of DMSO deemed to have no inhibitory effects when tested in prior assays. In each assay, the extract to be tested was diluted from stock to concentrations of 500, 250, 125, 62.5 and $31.25 \mu \mathrm{g} / \mathrm{ml}$ in $100 \mu \mathrm{l}$ LB. Also in each assay, one of three antibiotics: ampicillin, amikacin or G418 sulfate, was diluted in LB to a series of concentrations previously determined to possess intermediate antibacterial efficacy versus Staphylococcus aureus under similar assay conditions (data not shown). 
Each extract concentration and each antibiotic concentration was tested independently, as was each possible extract/antibiotic combination. All samples, including controls, were prepared in triplicate, and each assay was run in triplicate. Averaged initial $\mathrm{OD}_{600}$ values, following sample treatment and bacterial inoculation, but prior to incubation, were subtracted from all subsequent readings to account for background absorbance of the media, antibiotics and extracts. Negative values were considered as readings of zero. Plates were incubated at approximately $37^{\circ} \mathrm{C}$ and 125 $\mathrm{rpm}$, with additional $\mathrm{OD}_{600}$ measurements taken at 1-hour intervals for 6 hours using a PowerWave XS Microplate Spectrophotometer (Bio-Tek). The averaged results from each of the three assays were then combined and averaged together. Statistical analysis was performed on the 6-hour time point values using an unpaired t-test assuming equal variances. $\mathrm{P}$ values of less than 0.05 were considered statistically significant.

The first set of assays tested the hexane extract, alone and in combination with the antibiotic G418 sulfate. As can be seen in figure 7, the extract at a concentration of 500 $\mu \mathrm{g} / \mathrm{ml}$ demonstrated significant antibacterial efficacy through 6 hours, with a growth curve similar to that of the positive control, while a concentration of $250 \mu \mathrm{g} / \mathrm{ml}$ also resulted in moderate, but significant growth inhibition. The growth curves of the samples treated at lower extract concentrations more closely resembled those of the untreated control, with no statistically significant bacterial inhibition. 


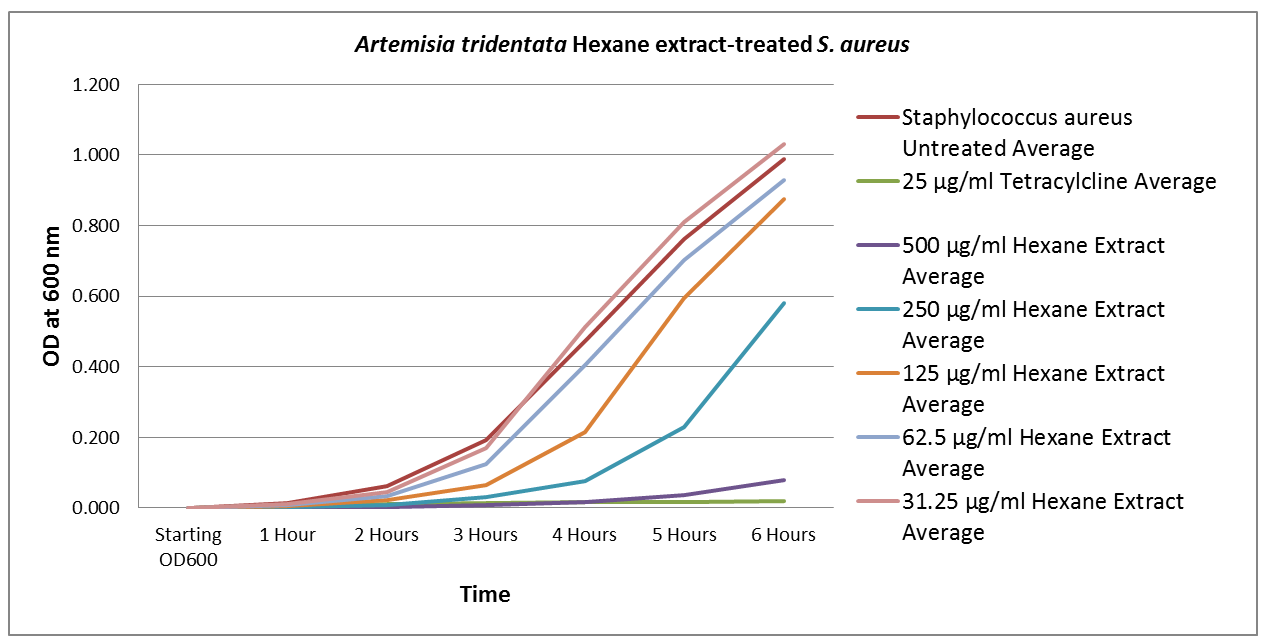

Figure 7: S. aureus treated with A. tridentata hexane extract

As can be seen in figure 8, G418 sulfate demonstrated strong antibacterial efficacy at a concentration of $10 \mu \mathrm{g} / \mathrm{ml}$, with moderate effects seen at the $5 \mu \mathrm{g} / \mathrm{ml}$ level, and mild effects at a concentration of $2.5 \mu \mathrm{g} / \mathrm{ml}$, with all results statistically significant. G418 sulfate at a concentration of $1.25 \mu \mathrm{g} / \mathrm{ml}$ failed to significantly inhibit bacterial growth.

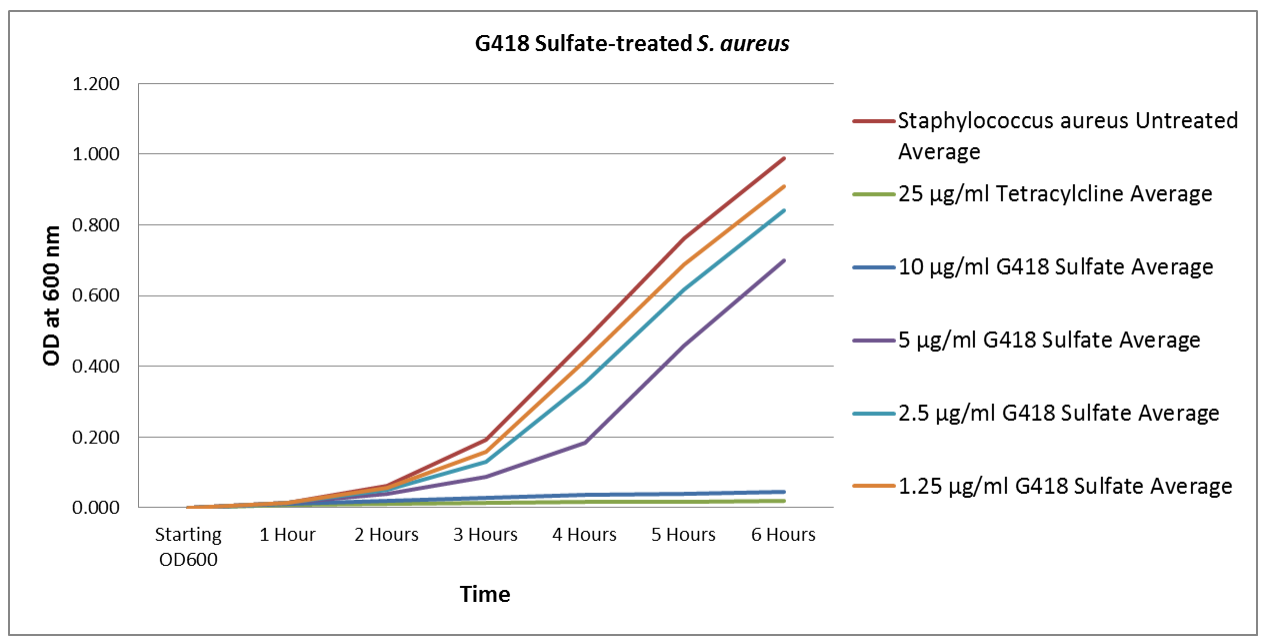

Figure 8: S. aureus treated with G418 sulfate 
As can be seen in figures 9-13, the combination of hexane extract with G418 sulfate enhanced growth inhibition. At a G418 sulfate concentration of $1.25 \mu \mathrm{g} / \mathrm{ml}$ for example, growth inhibition became significant in comparison to the untreated control when combined with extract concentrations of $62.5 \mu \mathrm{g} / \mathrm{ml}$ and greater, and in comparison to the antibiotic alone at this concentration when combined with extract levels of $125 \mu \mathrm{g} / \mathrm{ml}$ and greater.

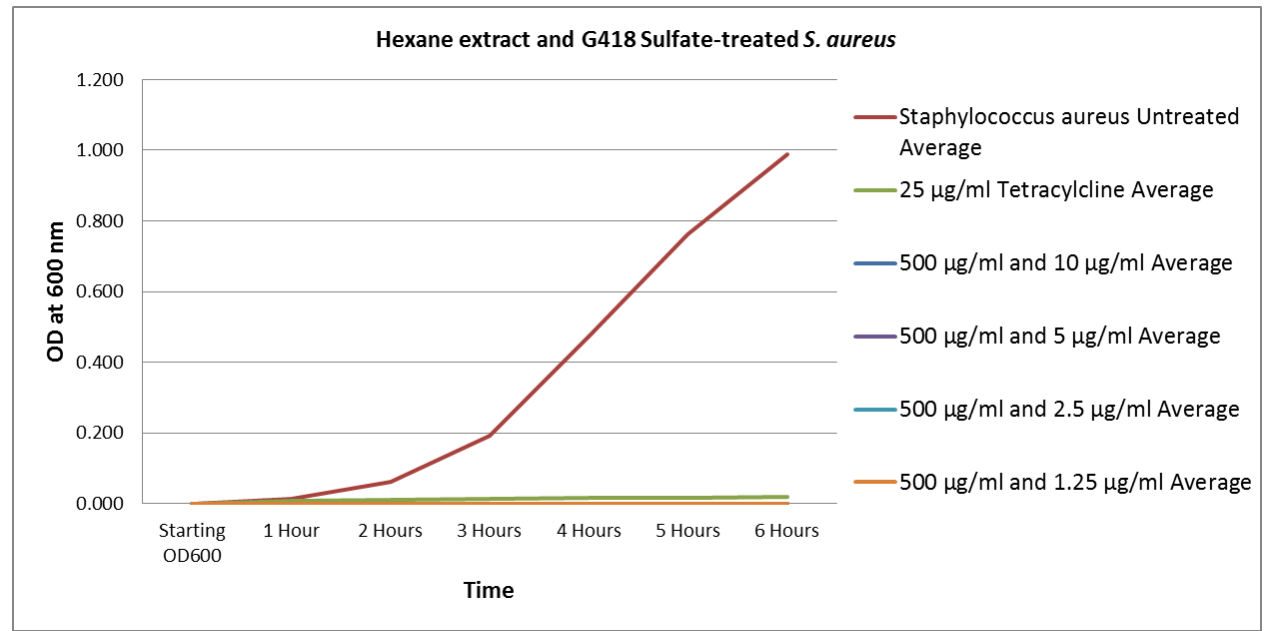

Figure 9: S. aureus treated with G418 sulfate and hexane extract $(500 \mu \mathrm{g} / \mathrm{ml})$

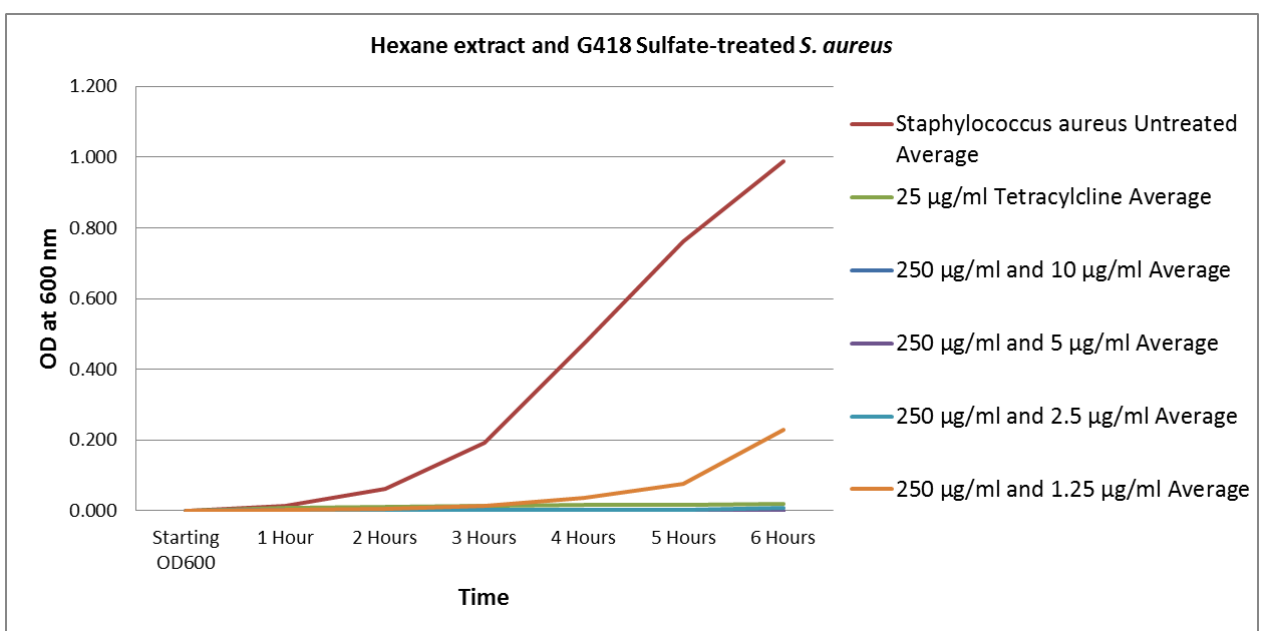

Figure 10: S. aureus treated with G418 sulfate and hexane extract $(250 \mu \mathrm{g} / \mathrm{ml})$ 


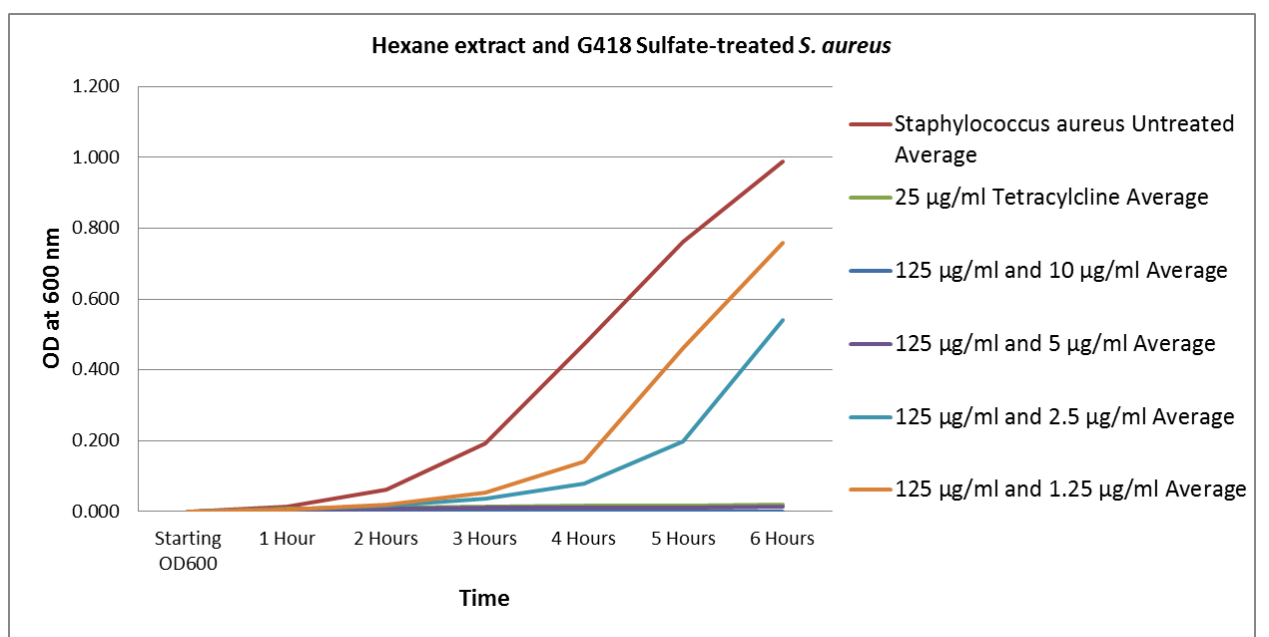

Figure 11: S. aureus treated with G418 sulfate and hexane extract $(125 \mu \mathrm{g} / \mathrm{ml})$

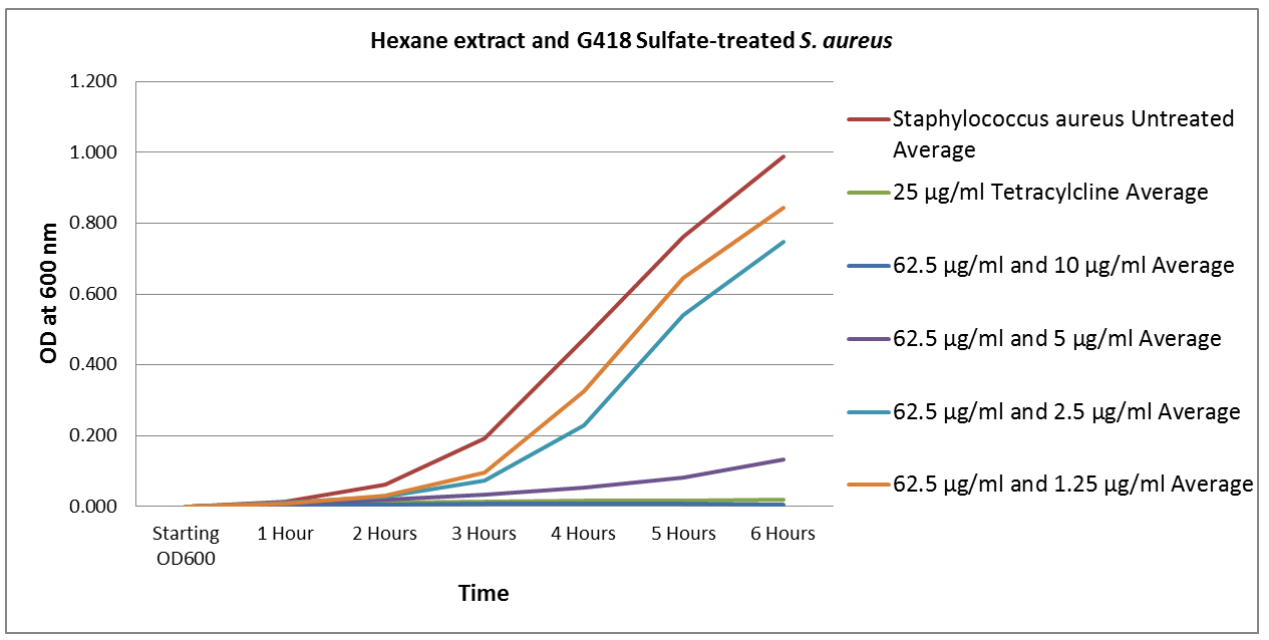

Figure 12: S. aureus treated with G418 sulfate and hexane extract $(62.5 \mu \mathrm{g} / \mathrm{ml})$ 


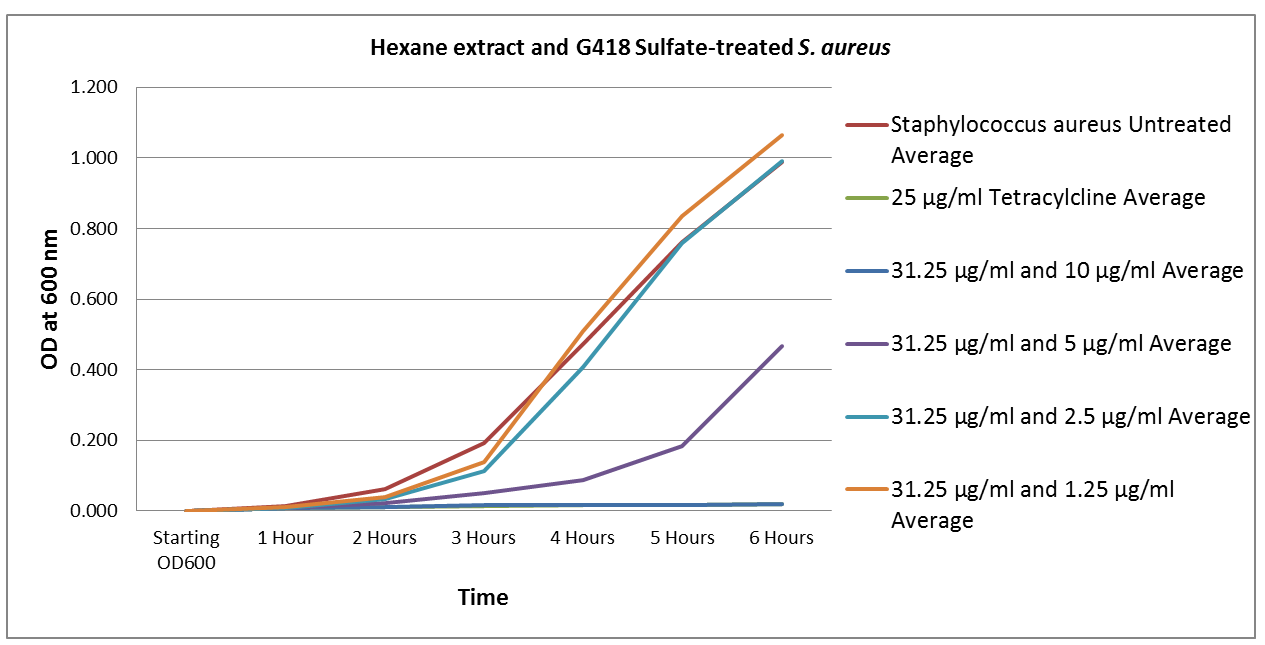

Figure 13: S. aureus treated with G418 sulfate and hexane extract $(31.25 \mu \mathrm{g} / \mathrm{ml})$

The next set of assays tested the hexane extract alone and in combination with the antibiotic amikacin. As can be seen in figure 14, the results for the hexane extract alone were very similar to those of the G418 sulfate combinatorial assays, with significant antibacterial effects only at concentrations of 250 and $500 \mu \mathrm{g} / \mathrm{ml}$. Amikacin demonstrated strong efficacy at concentrations of 10 and $5 \mu \mathrm{g} / \mathrm{ml}$, with lesser, but still statistically significant growth inhibition at a concentration of $2.5 \mu \mathrm{g} / \mathrm{ml}$ (figure 15).

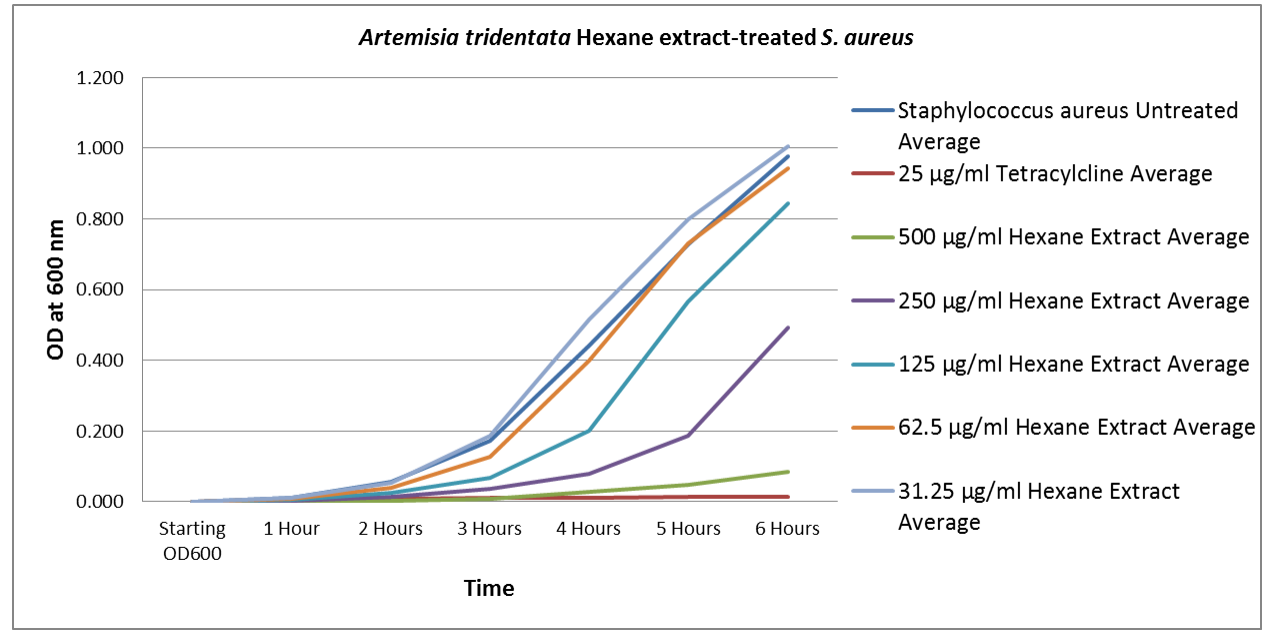

Figure 14: S. aureus treated with A. tridentata hexane extract 


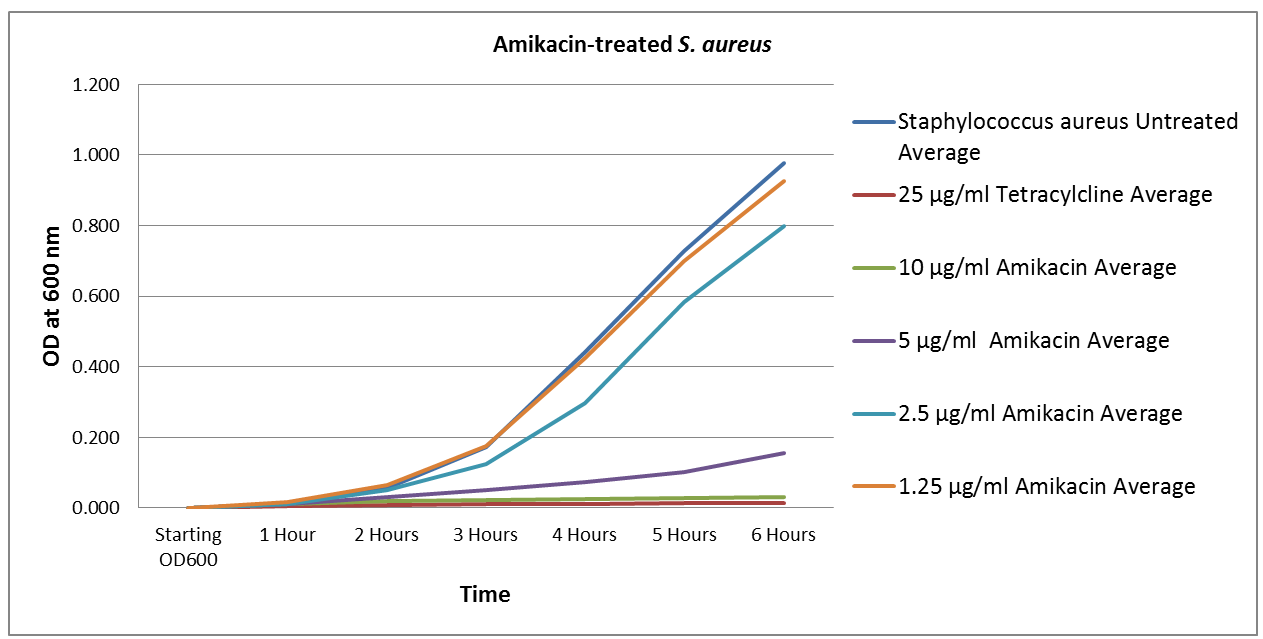

Figure 15: S. aureus treated with amikacin

In combination (figures 16-20), the efficacy of growth inhibition was again visibly improved. Growth inhibition by amikacin at a concentration of $1.25 \mu \mathrm{g} / \mathrm{ml}$ became statistically significant compared to the untreated control when combined with extract at concentrations of $62.5 \mu \mathrm{g} / \mathrm{ml}$ and greater, and in comparison to the antibiotic alone at this concentration at extract levels of $125 \mu \mathrm{g} / \mathrm{ml}$ and greater.

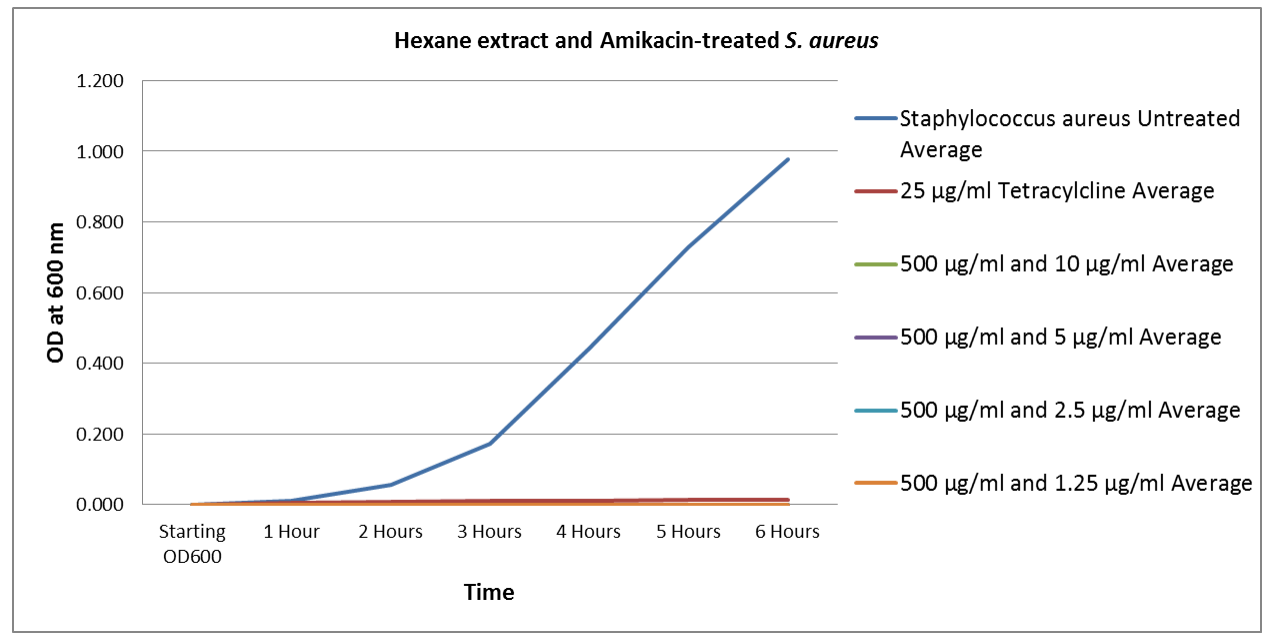

Figure 16: S. aureus treated with amikacin and hexane extract $(500 \mu \mathrm{g} / \mathrm{ml})$ 


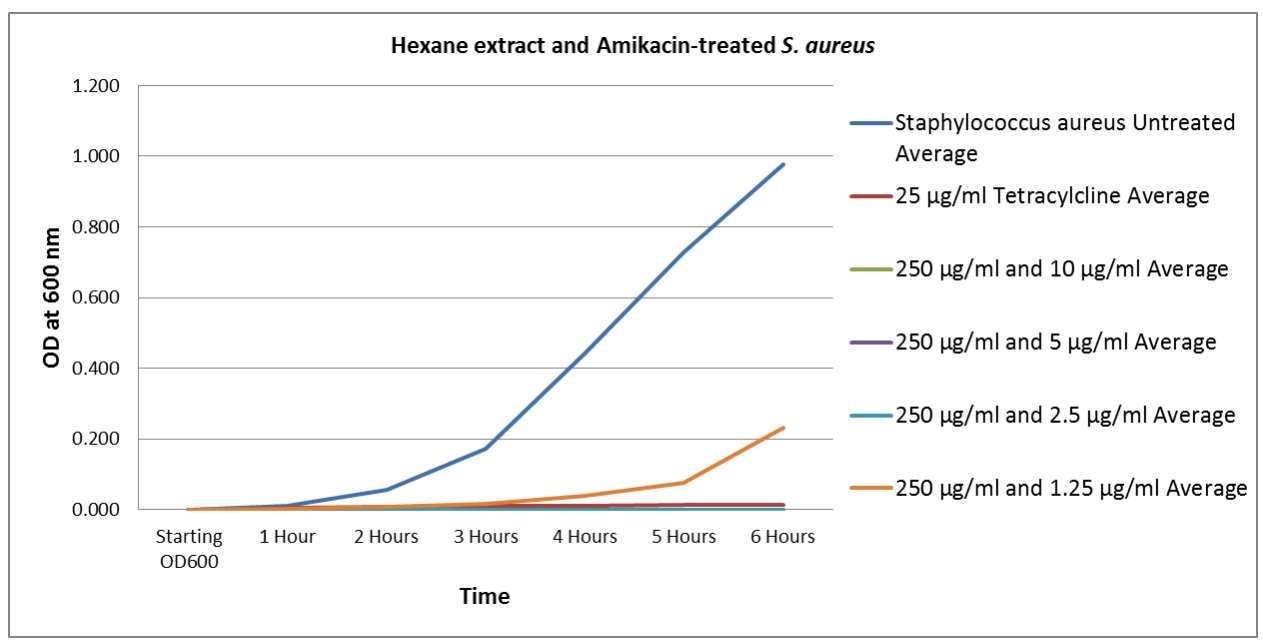

Figure 17: S. aureus treated with amikacin and hexane extract $(250 \mu \mathrm{g} / \mathrm{ml})$

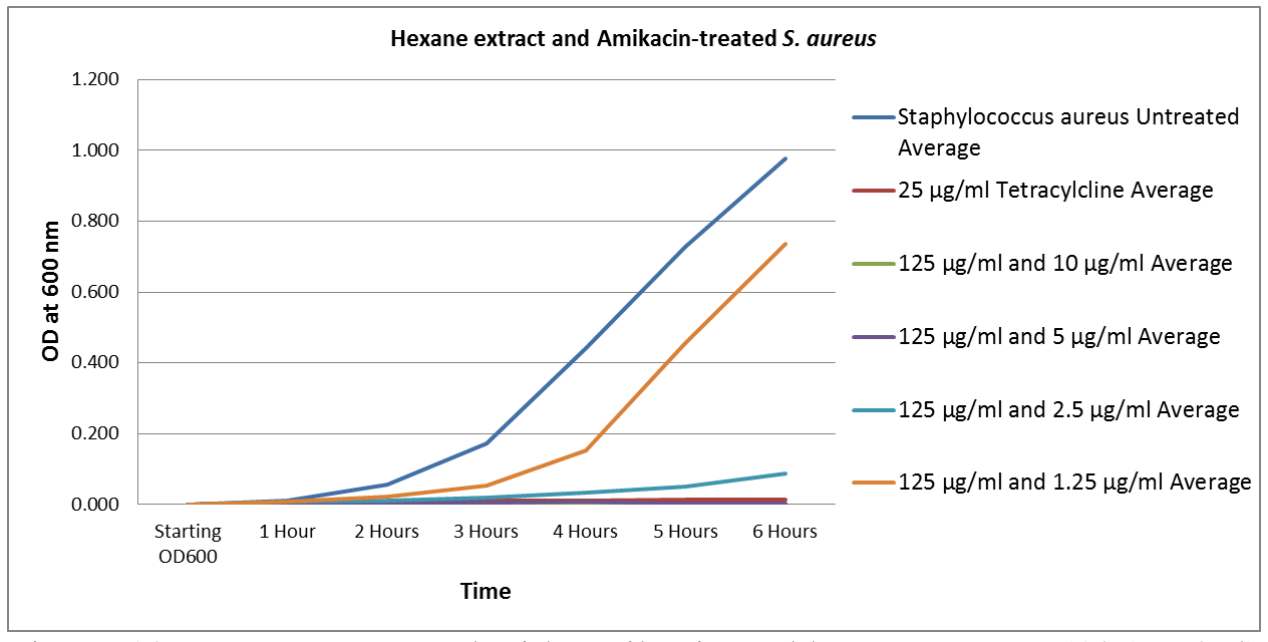

Figure 18: S. aureus treated with amikacin and hexane extract $(125 \mu \mathrm{g} / \mathrm{ml})$ 


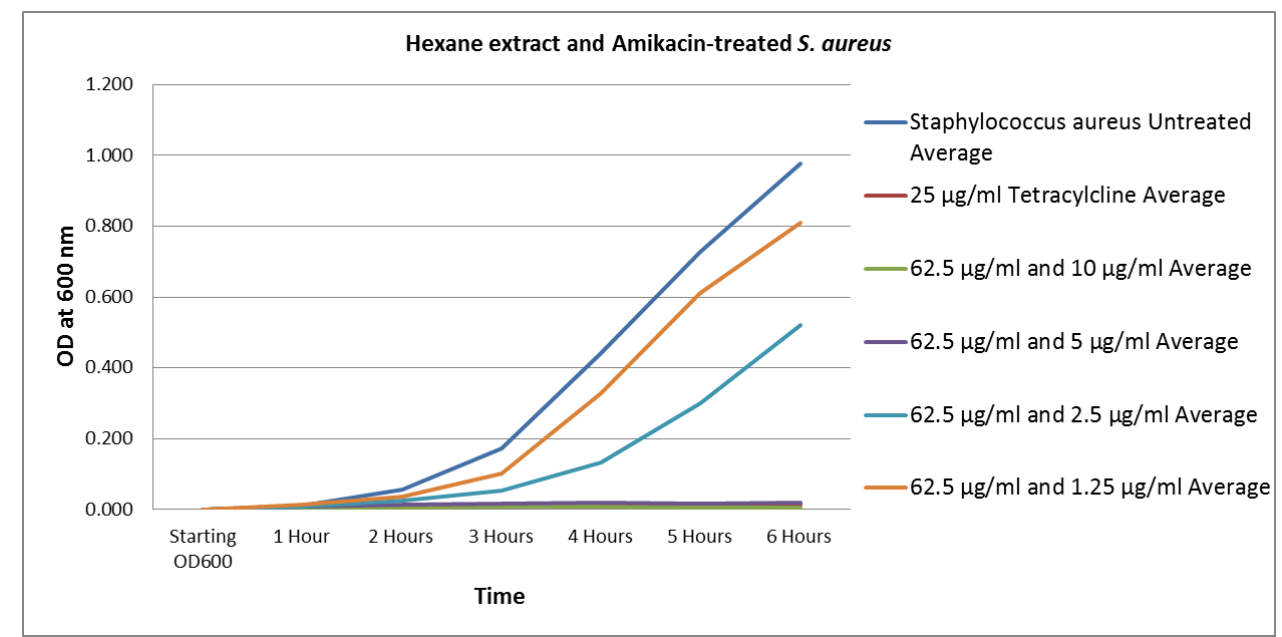

Figure 19: S. aureus treated with amikacin and hexane extract $(62.5 \mu \mathrm{g} / \mathrm{ml})$

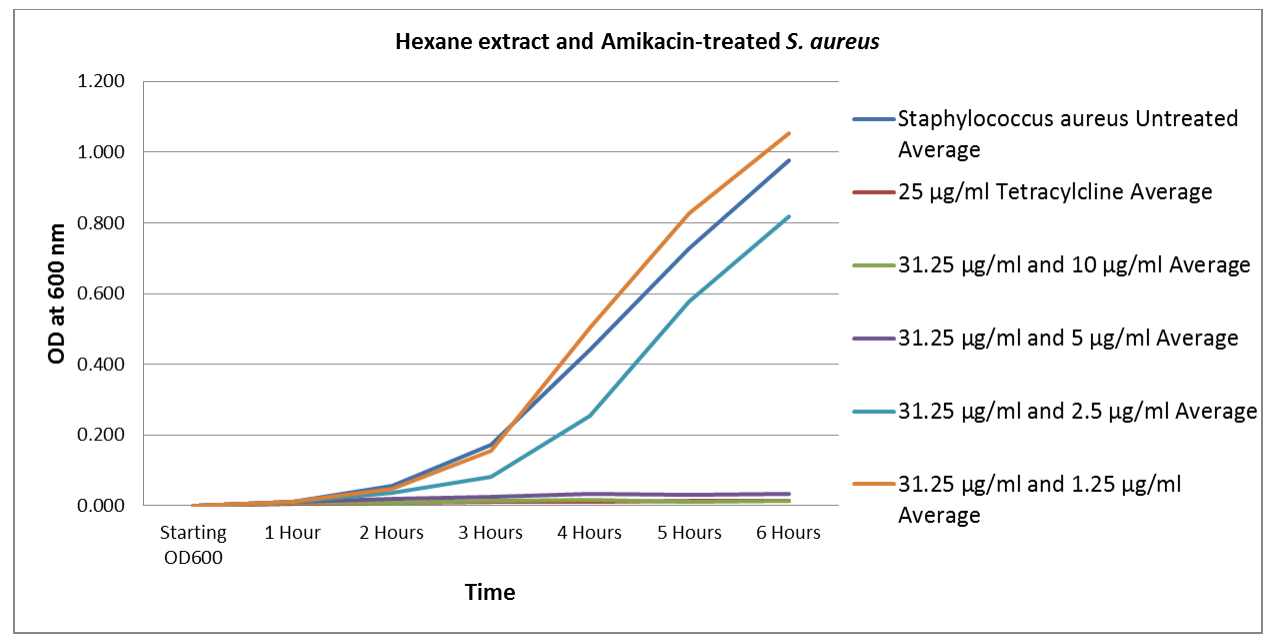

Figure 20: S. aureus treated with amikacin and hexane extract $(31.25 \mu \mathrm{g} / \mathrm{ml})$

The next set of assays tested the hexane extract alone and in combination with the antibiotic ampicillin. As can be seen in figure 21, the results for the hexane extract alone were very similar to those of the previous assays, though inhibition at the concentration of $125 \mu \mathrm{g} / \mathrm{ml}$ was also statistically significant in these assays. Growth inhibition by 
ampicillin (figure 22), though not comparable to the positive control, was statistically significant at all concentrations tested.

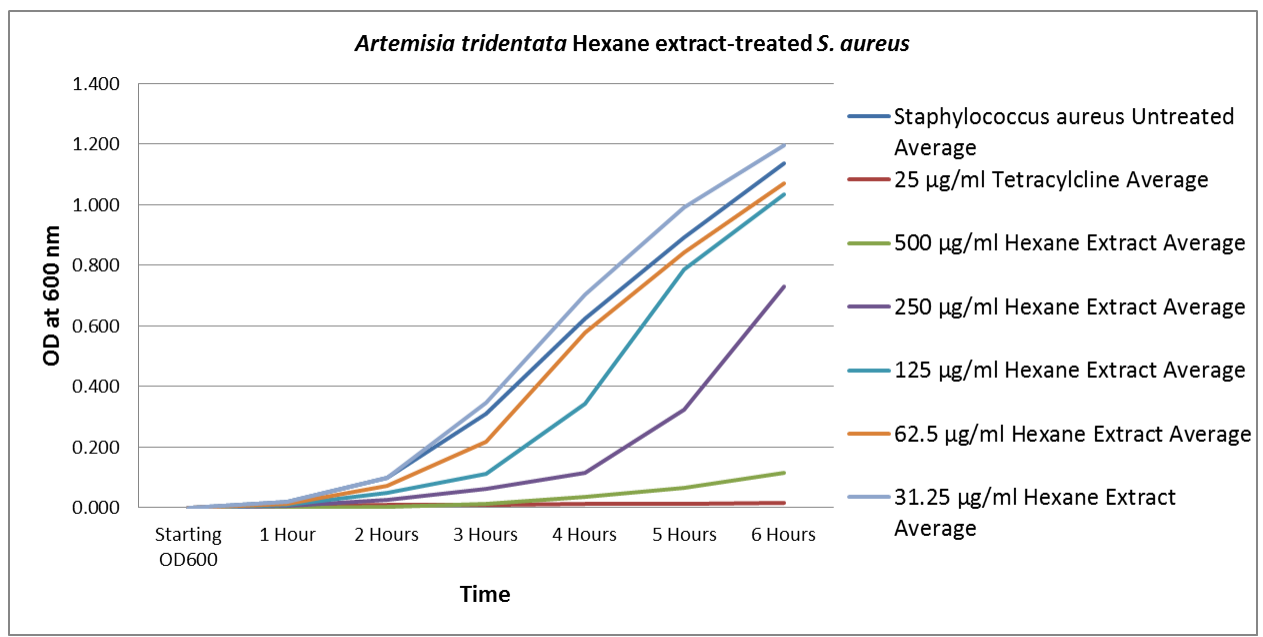

Figure 21: S. aureus treated with A. tridentata hexane extract

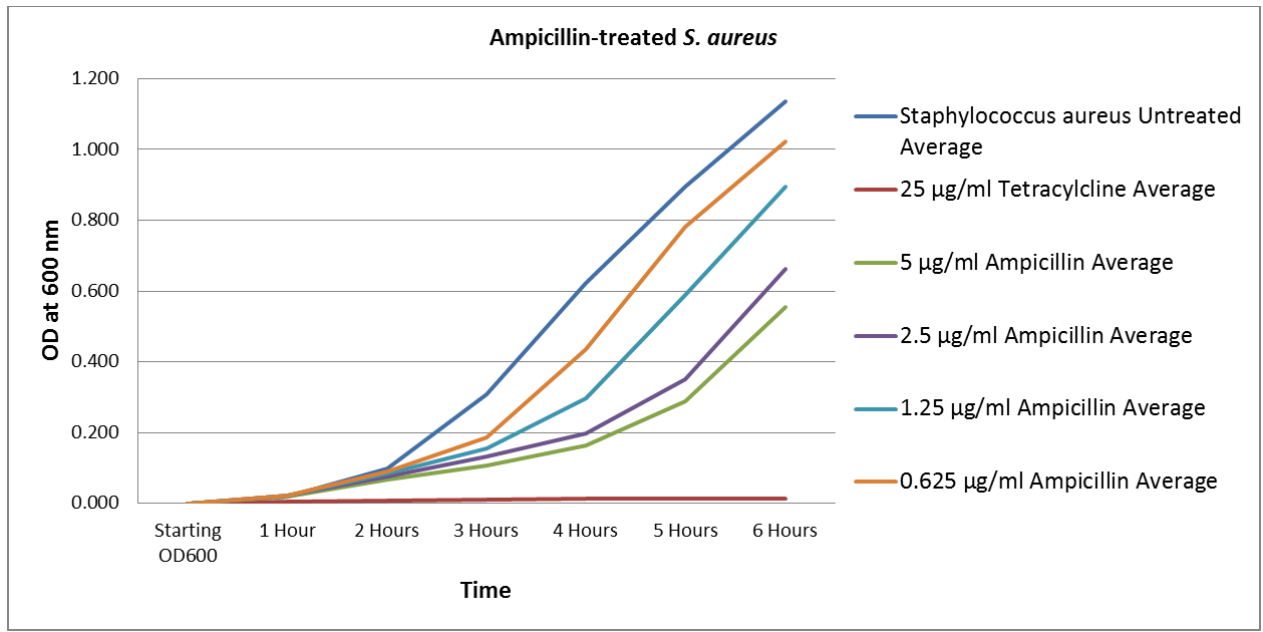

Figure 22: S. aureus treated with ampicillin

In combination (figures 23-27), antibacterial efficacy was again enhanced. The lower concentrations of 0.625 and $1.25 \mu \mathrm{g} / \mathrm{ml}$ for example, showed significantly 
improved efficacy in comparison to their individual performances when combined with extract levels of $125 \mu \mathrm{g} / \mathrm{ml}$ or greater.

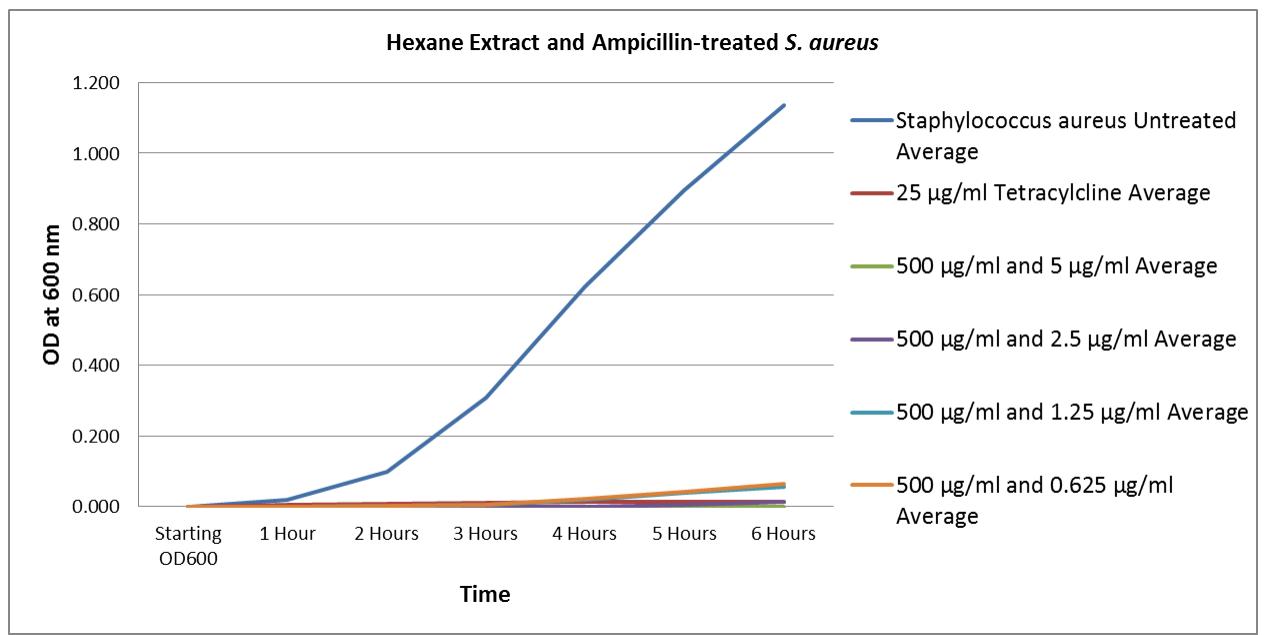

Figure 23: S. aureus treated with ampicillin and hexane extract $(500 \mu \mathrm{g} / \mathrm{ml})$

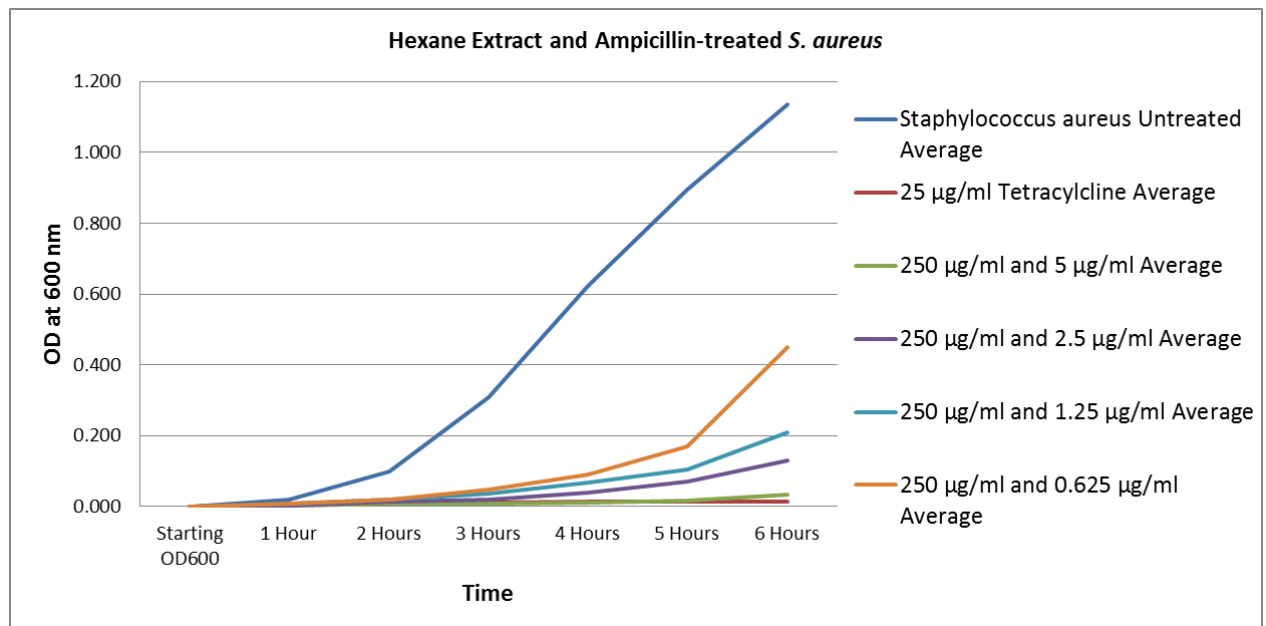

Figure 24: S. aureus treated with ampicillin and hexane extract $(250 \mu \mathrm{g} / \mathrm{ml})$ 


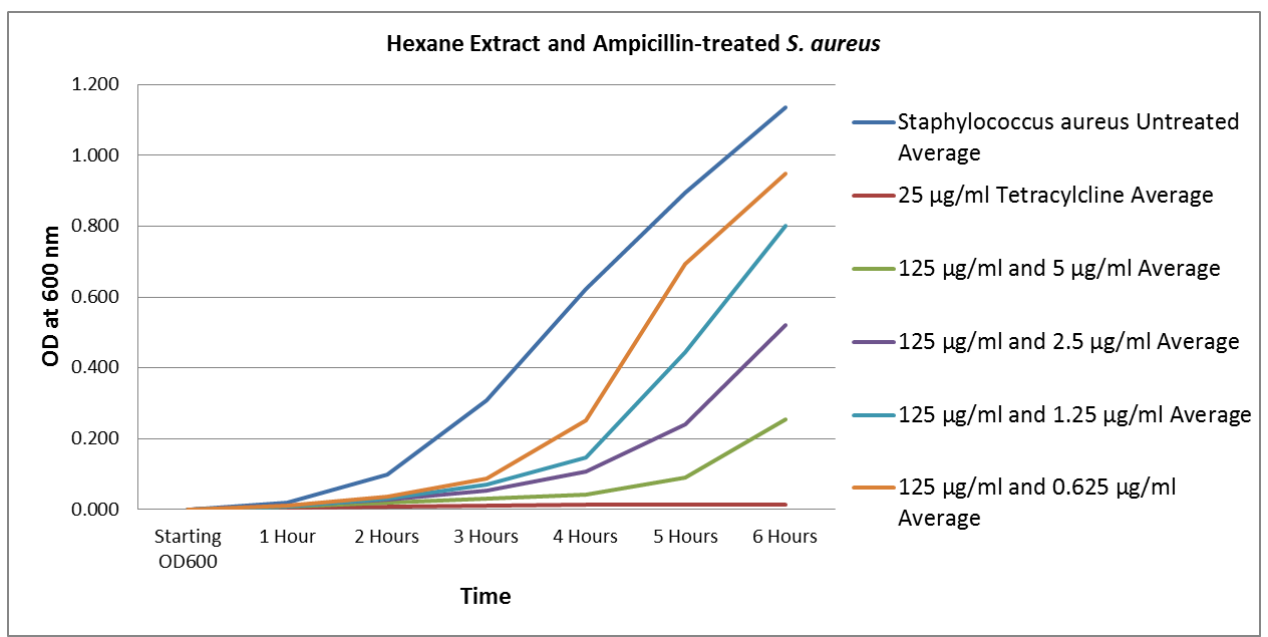

Figure 25: S. aureus treated with ampicillin and hexane extract $(125 \mu \mathrm{g} / \mathrm{ml})$

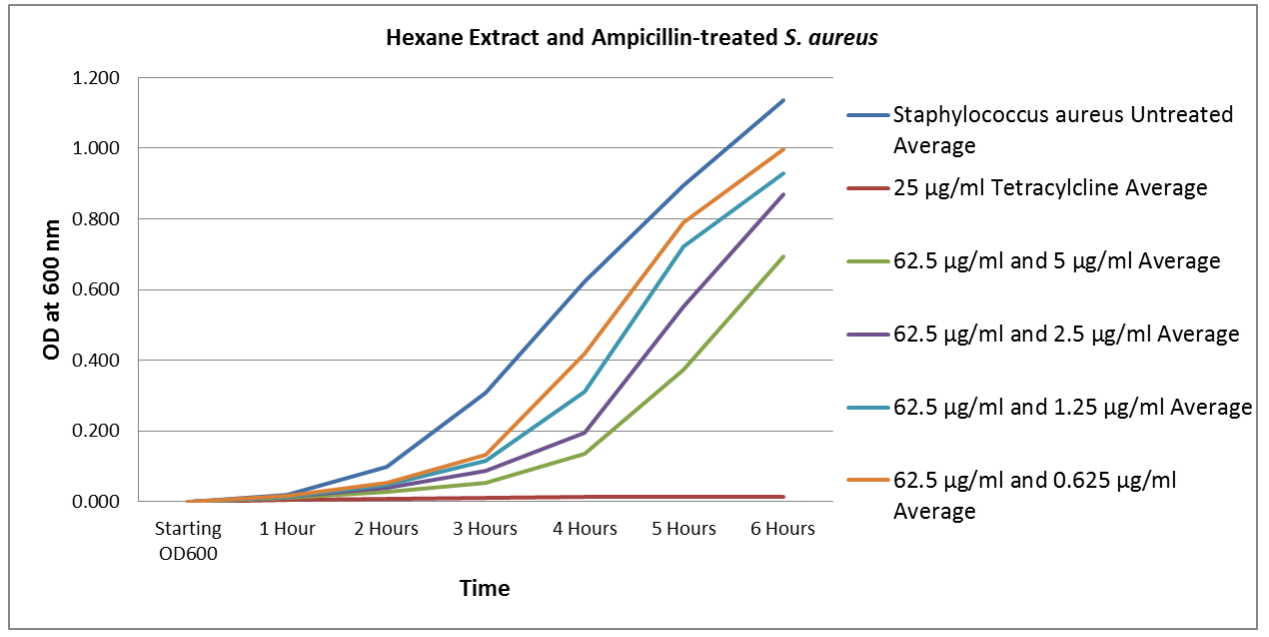

Figure 26: S. aureus treated with ampicillin and hexane extract $(62.5 \mu \mathrm{g} / \mathrm{ml})$ 


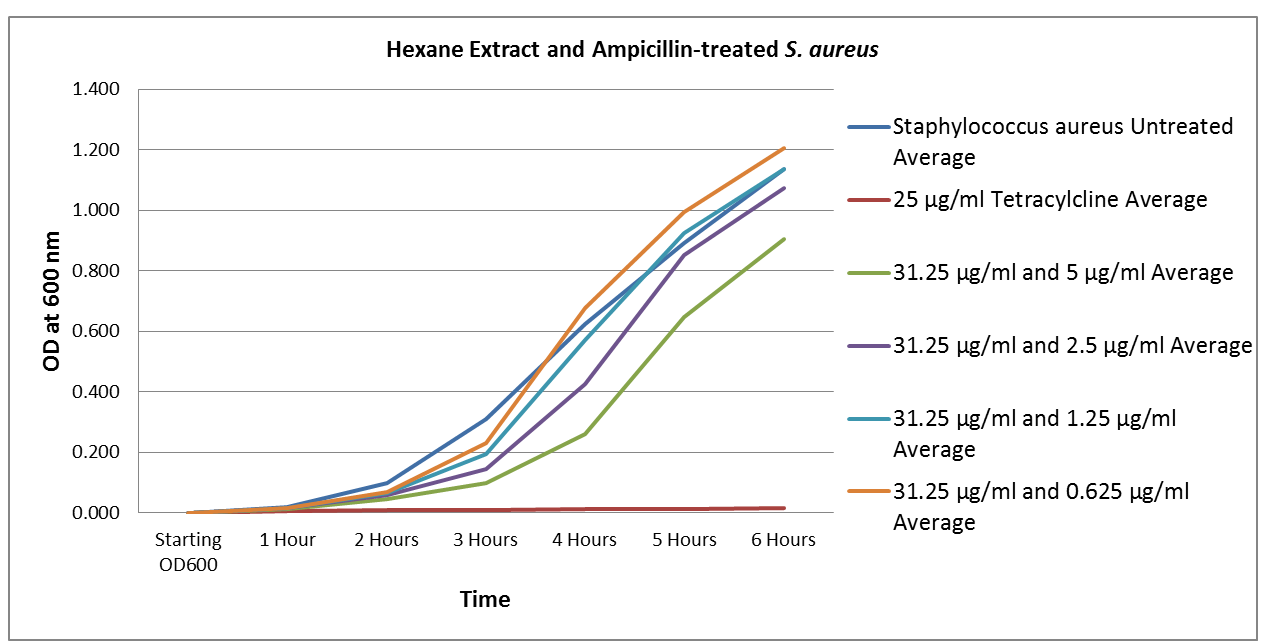

Figure 27: S. aureus treated with ampicillin and hexane extract $(31.25 \mu \mathrm{g} / \mathrm{ml})$

The ethyl acetate extract was the next to be assayed in combination with these antibiotics, beginning with G418 sulfate. As can be seen in figure 28, the ethyl acetate extract alone displayed stronger antibacterial efficacy than did the hexane extract, with statistically significant growth inhibition at concentrations as low as $62.5 \mu \mathrm{g} / \mathrm{ml}$. G418 sulfate performed as it did before (figure 29), with significant inhibition at levels of 2.5 $\mu \mathrm{g} / \mathrm{ml}$ and greater.

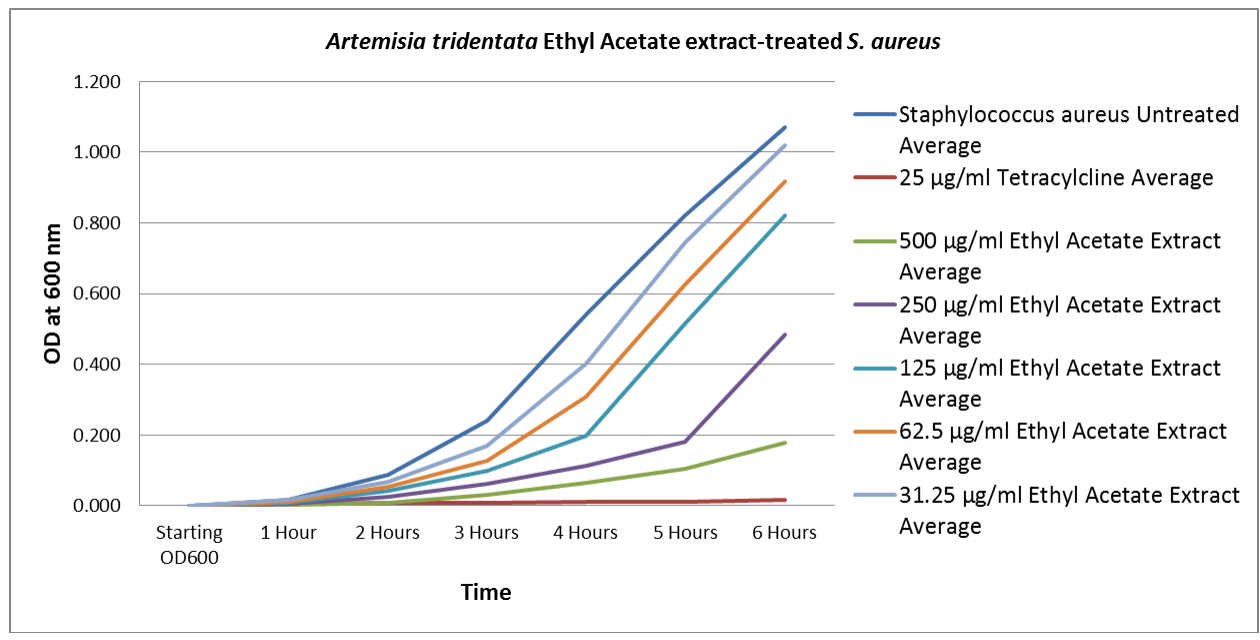

Figure 28: S. aureus treated with A. tridentata ethyl acetate extract 


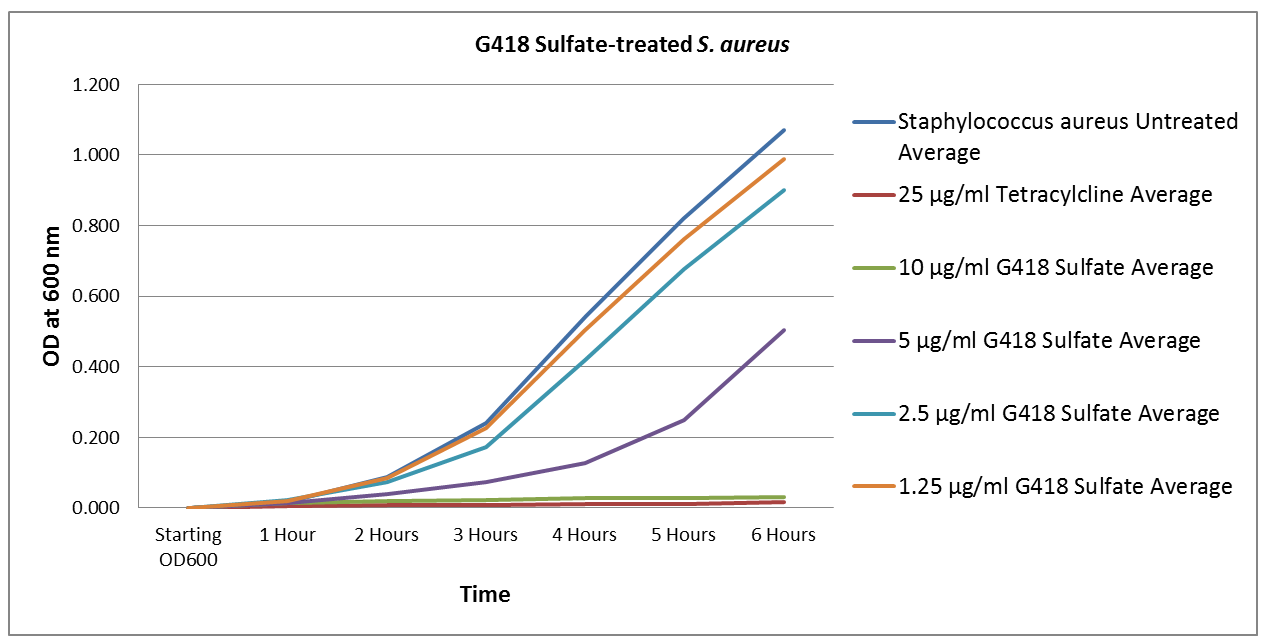

Figure 29: S. aureus treated with G418 sulfate

In combination (figures 30-34), G418 sulfate at a concentration of $1.25 \mu \mathrm{g} / \mathrm{ml}$, which alone was ineffective, exhibited significant growth inhibition in comparison to both the untreated control and the antibiotic alone at this level with extract concentrations as low as $62.5 \mu \mathrm{g} / \mathrm{ml}$. This combinatorial effect in comparison to the antibiotic alone is greater than that observed with the hexane extract and G418 sulfate.

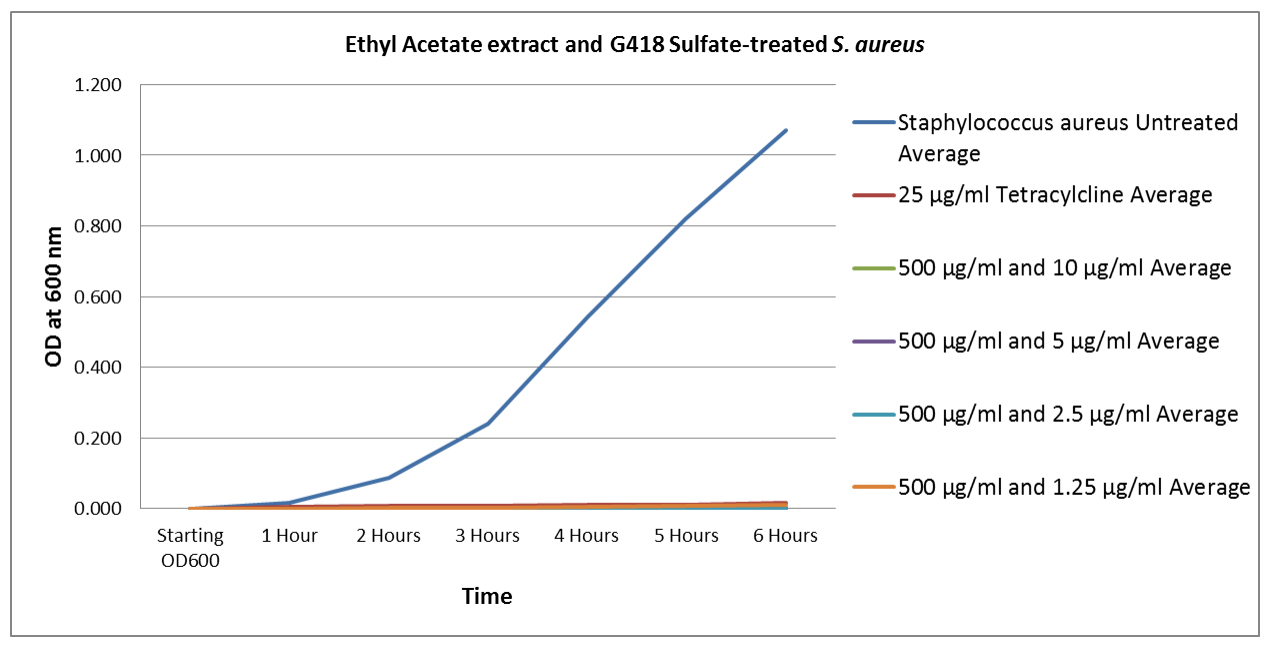

Figure 30: S. aureus treated with G418 sulfate and ethyl acetate extract $(500 \mu \mathrm{g} / \mathrm{ml})$ 


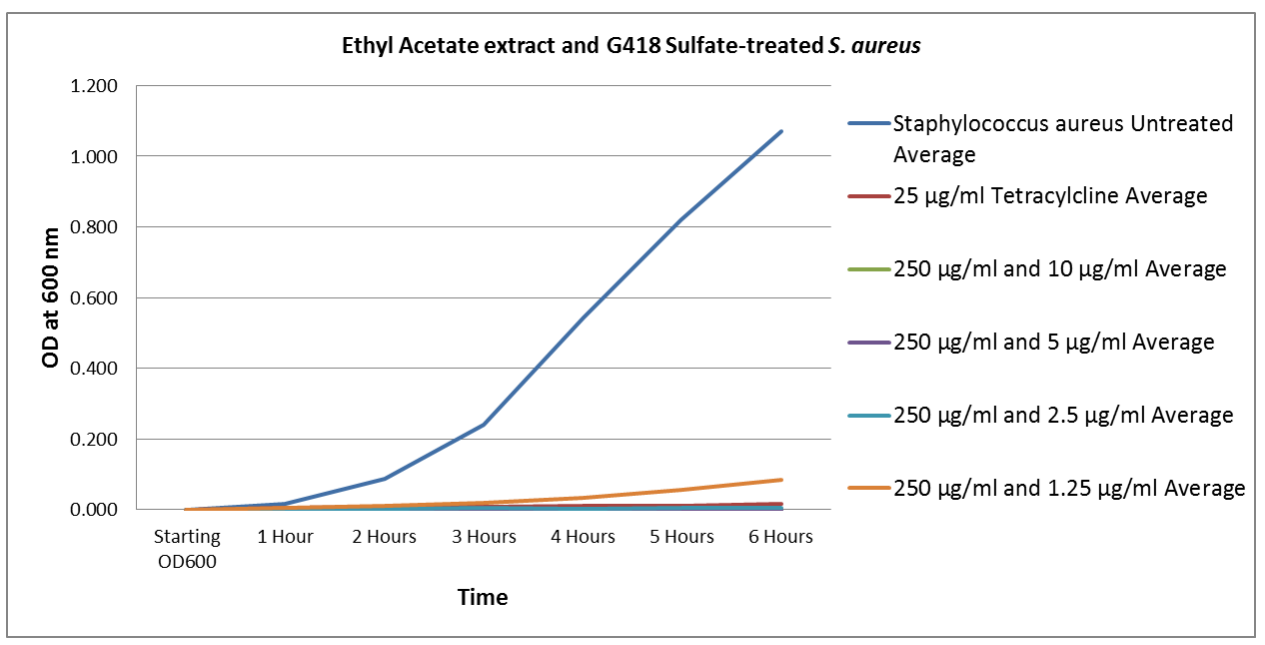

Figure 31: S. aureus treated with G418 sulfate and ethyl acetate extract $(250 \mu \mathrm{g} / \mathrm{ml})$

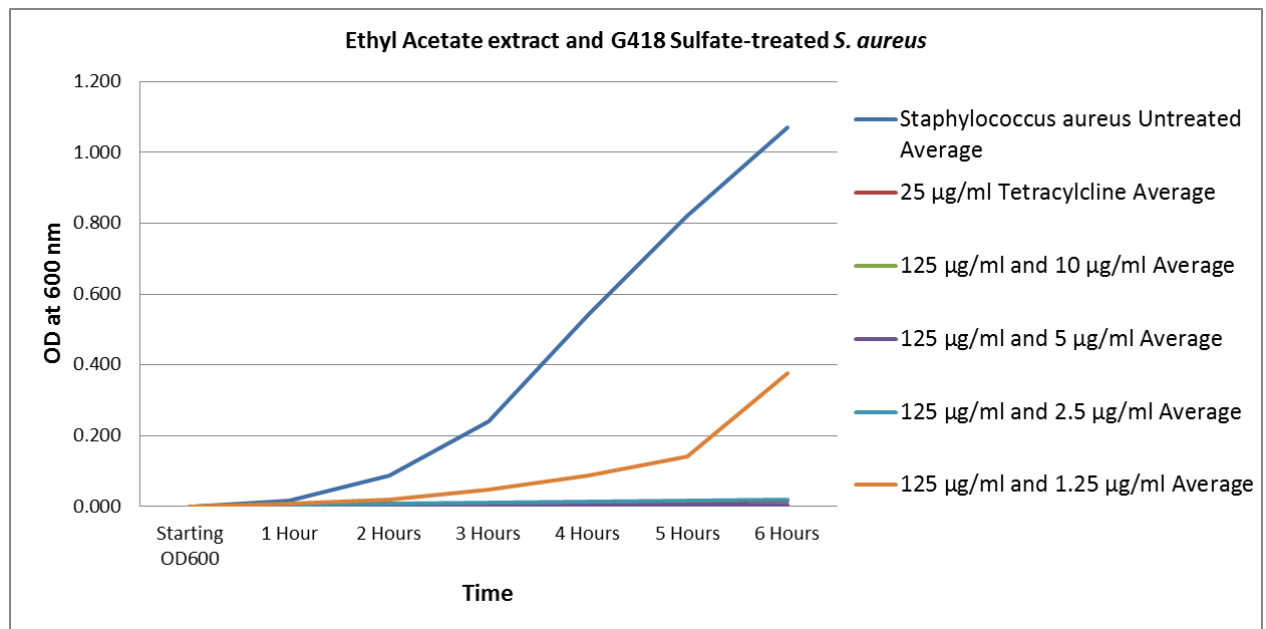

Figure 32: S. aureus treated with G418 sulfate and ethyl acetate extract $(125 \mu \mathrm{g} / \mathrm{ml})$ 


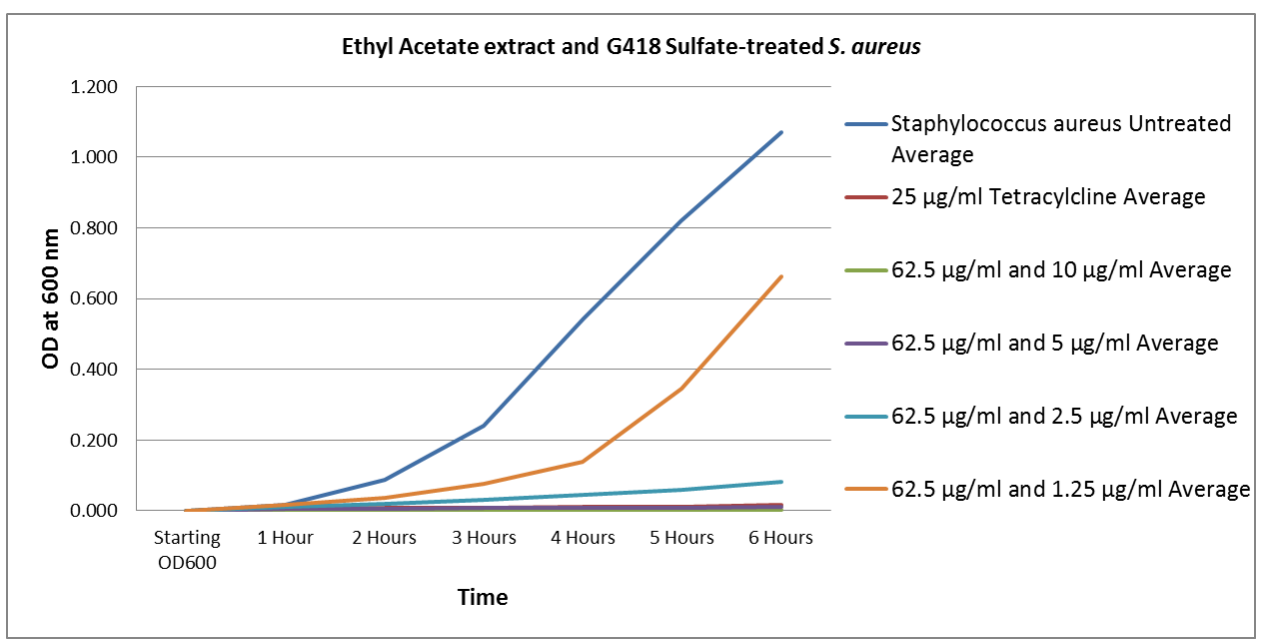

Figure 33: S. aureus treated with G418 sulfate and ethyl acetate extract $(62.5 \mu \mathrm{g} / \mathrm{ml})$

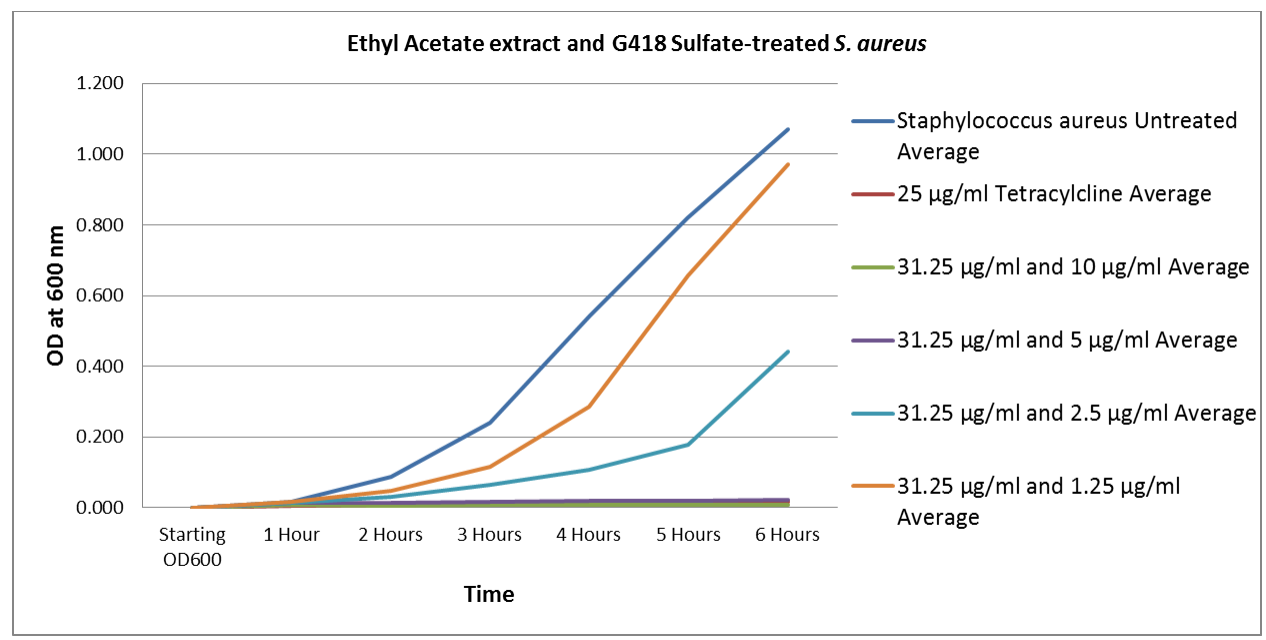

Figure 34: S. aureus treated with G418 sulfate and ethyl acetate extract $(31.25 \mu \mathrm{g} / \mathrm{ml})$

When tested alone (figures 35 and 36), both the ethyl acetate extract and amikacin performed as they had done previously, with significant inhibition seen at concentrations as low as $62.5 \mu \mathrm{g} / \mathrm{ml}$ by the extract, and at levels as low as $2.5 \mu \mathrm{g} / \mathrm{ml}$ for the antibiotic. 


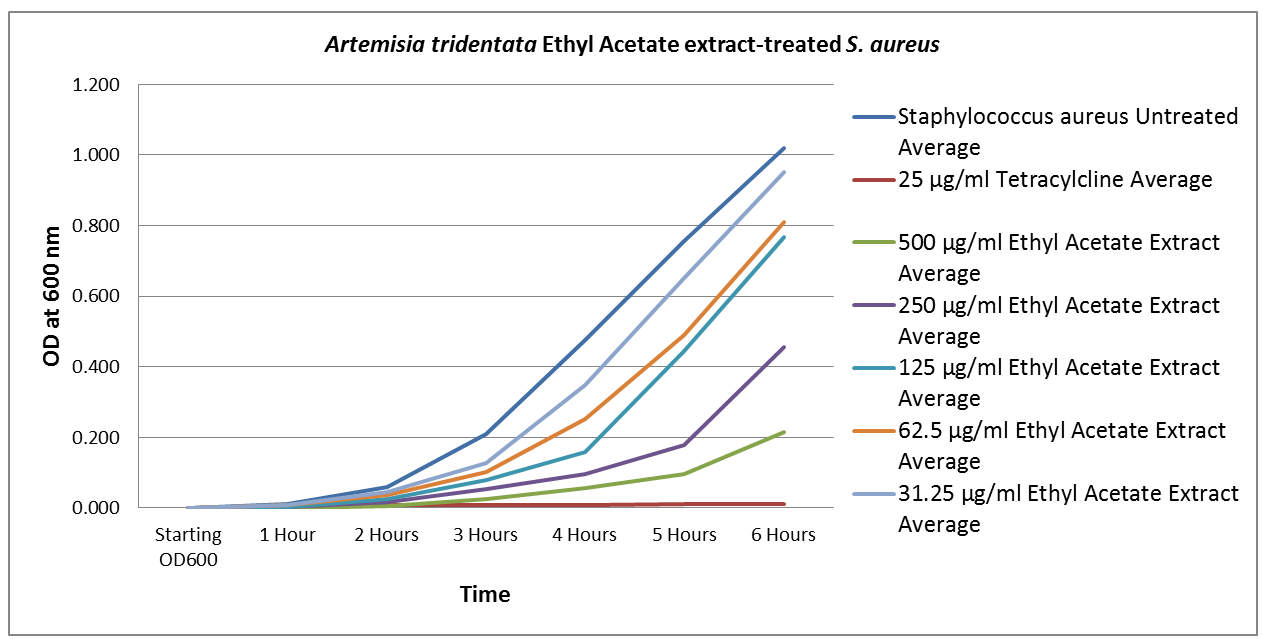

Figure 35: S. aureus treated with A. tridentata ethyl acetate extract

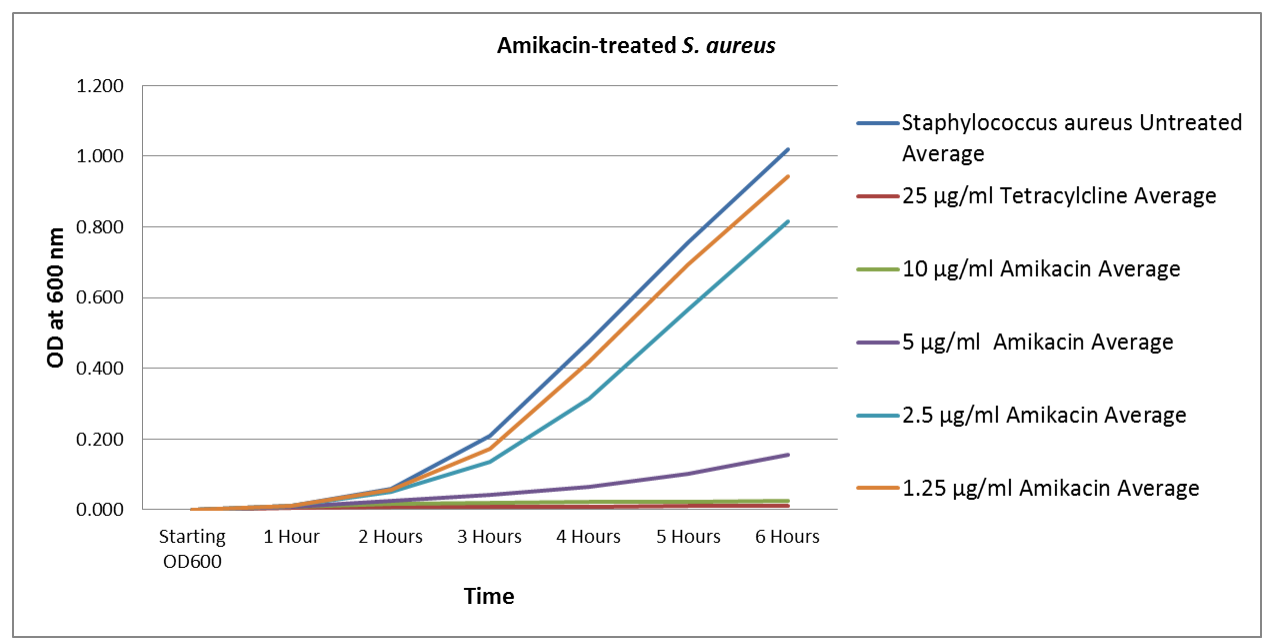

Figure 36: S. aureus treated with amikacin

At $1.25 \mu \mathrm{g} / \mathrm{ml}$ amikacin, growth inhibition became statistically significant in comparison to both the untreated control and the antibiotic alone at this level with extract concentrations of $62.5 \mu \mathrm{g} / \mathrm{ml}$ or greater (figures 37-41). Again, this combinatorial effect in comparison to the antibiotic alone was greater than that observed for amikacin with the hexane extract. 


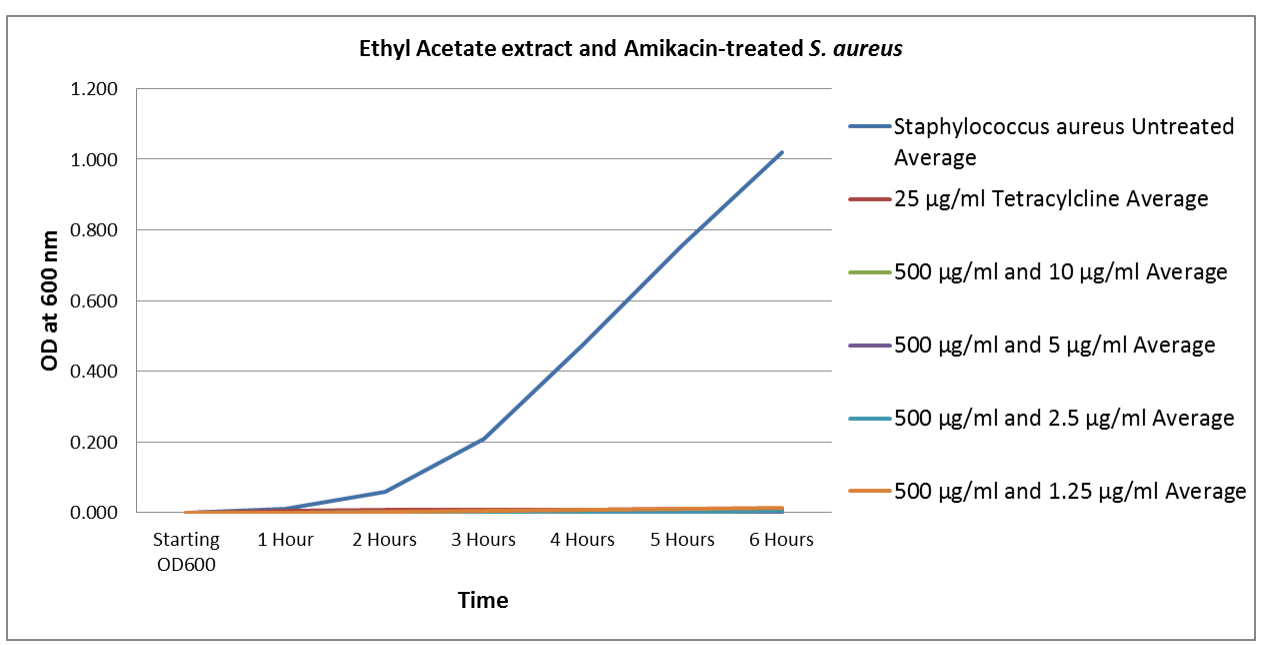

Figure 37: S. aureus treated with amikacin and ethyl acetate extract $(500 \mu \mathrm{g} / \mathrm{ml})$

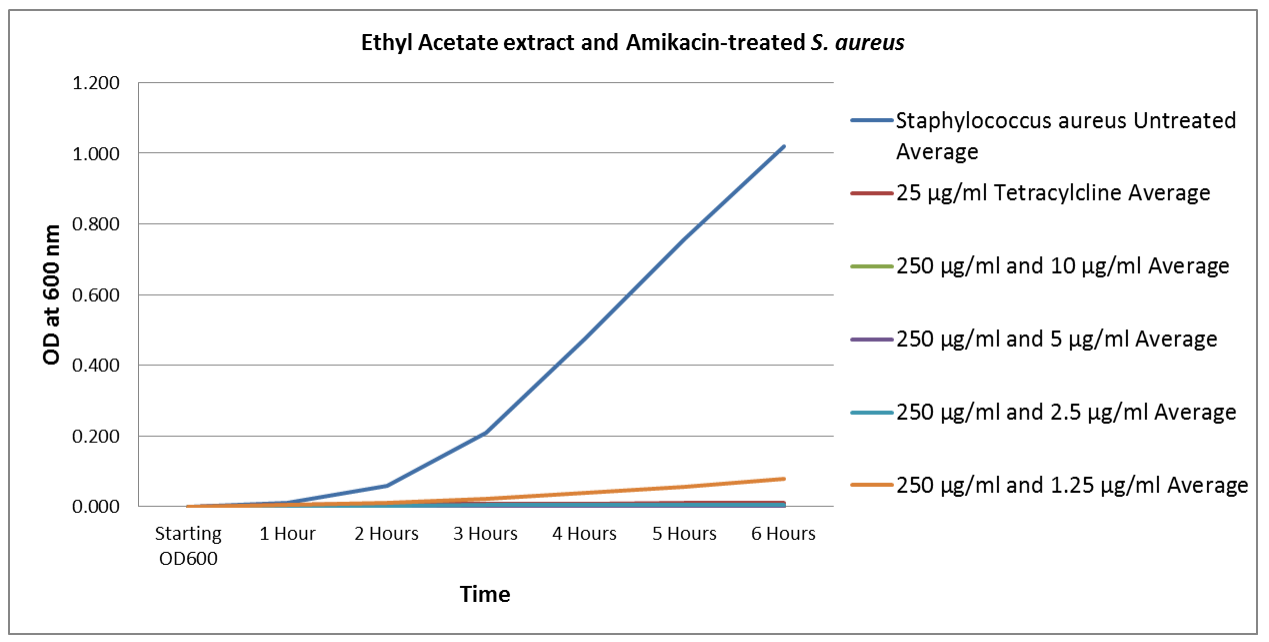

Figure 38: S. aureus treated with amikacin and ethyl acetate extract $(250 \mu \mathrm{g} / \mathrm{ml})$ 


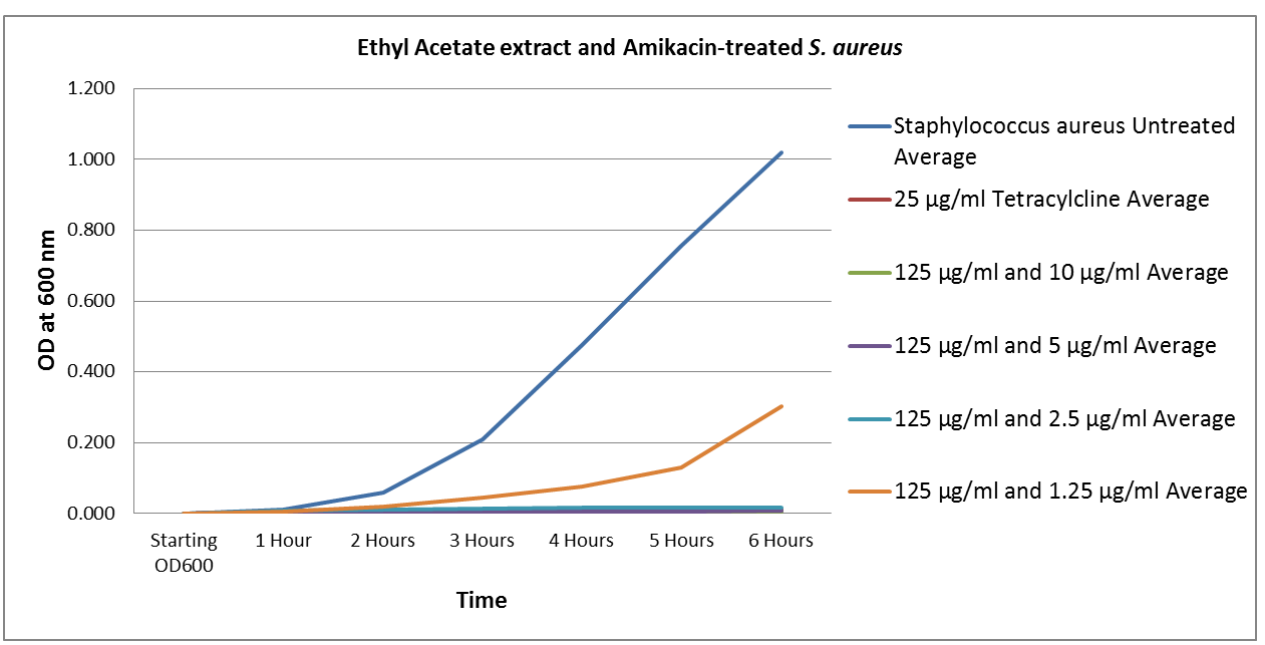

Figure 39: S. aureus treated with amikacin and ethyl acetate extract $(125 \mu \mathrm{g} / \mathrm{ml})$

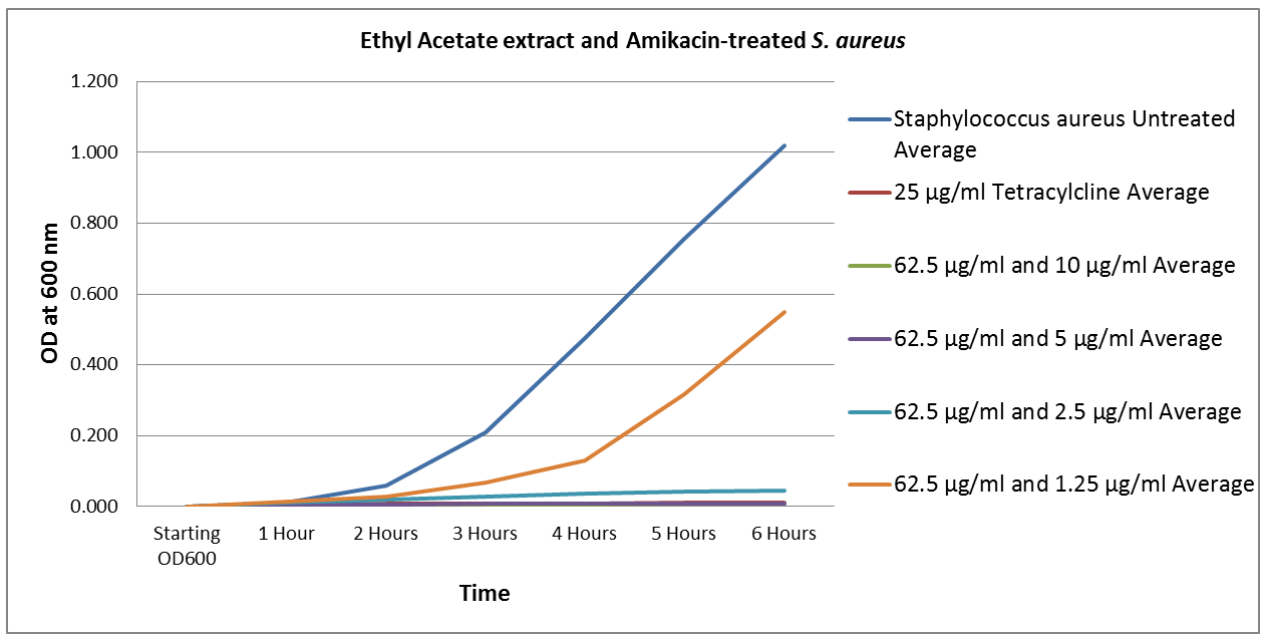

Figure 40: S. aureus treated with amikacin and ethyl acetate extract $(62.5 \mu \mathrm{g} / \mathrm{ml})$ 


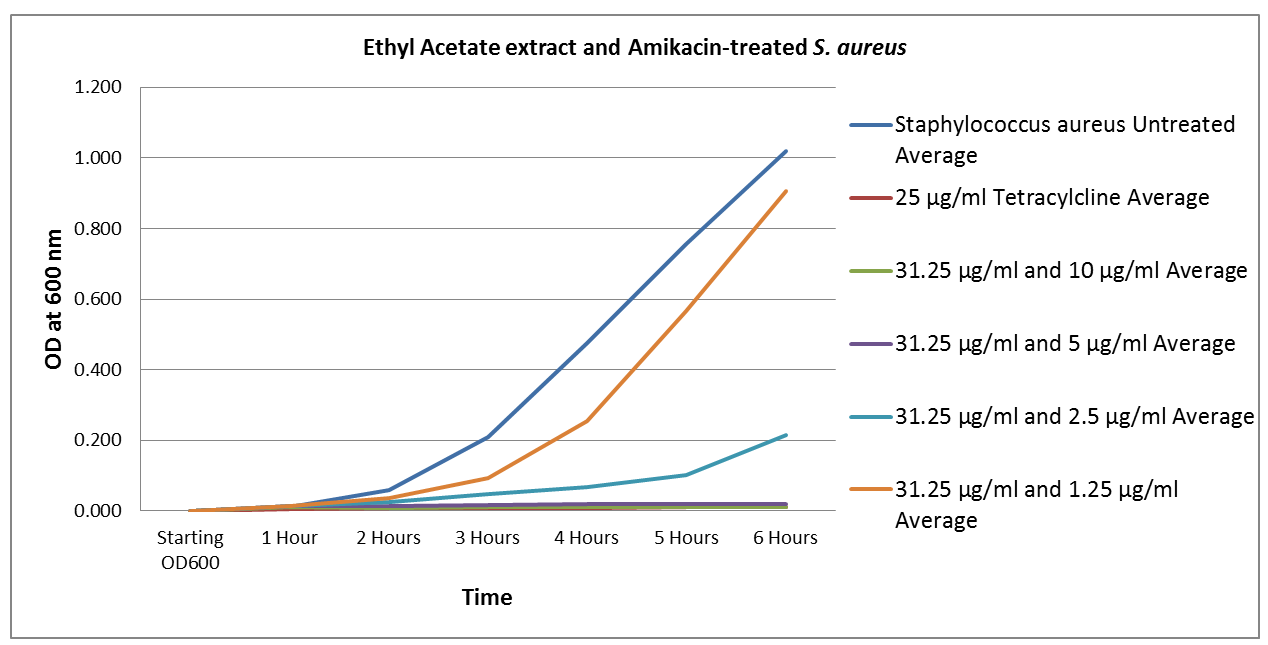

Figure 41: S. aureus treated with amikacin and ethyl acetate extract $(31.25 \mu \mathrm{g} / \mathrm{ml})$

When tested with ampicillin, the ethyl acetate extract (figure 42) again performed as before, with significant inhibition at concentrations as low as $62.5 \mu \mathrm{g} / \mathrm{ml}$, while the antibiotic (figure 43) again displayed significant antibacterial effects at all concentrations tested.

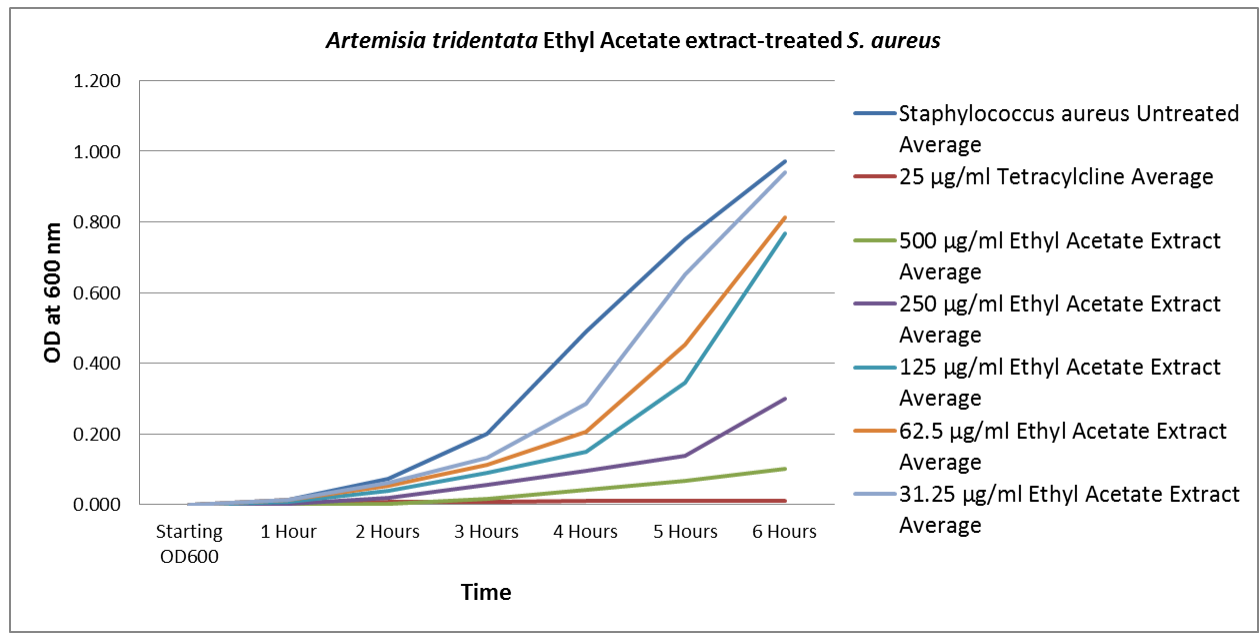

Figure 42: S. aureus treated with A. tridentata ethyl acetate extract 


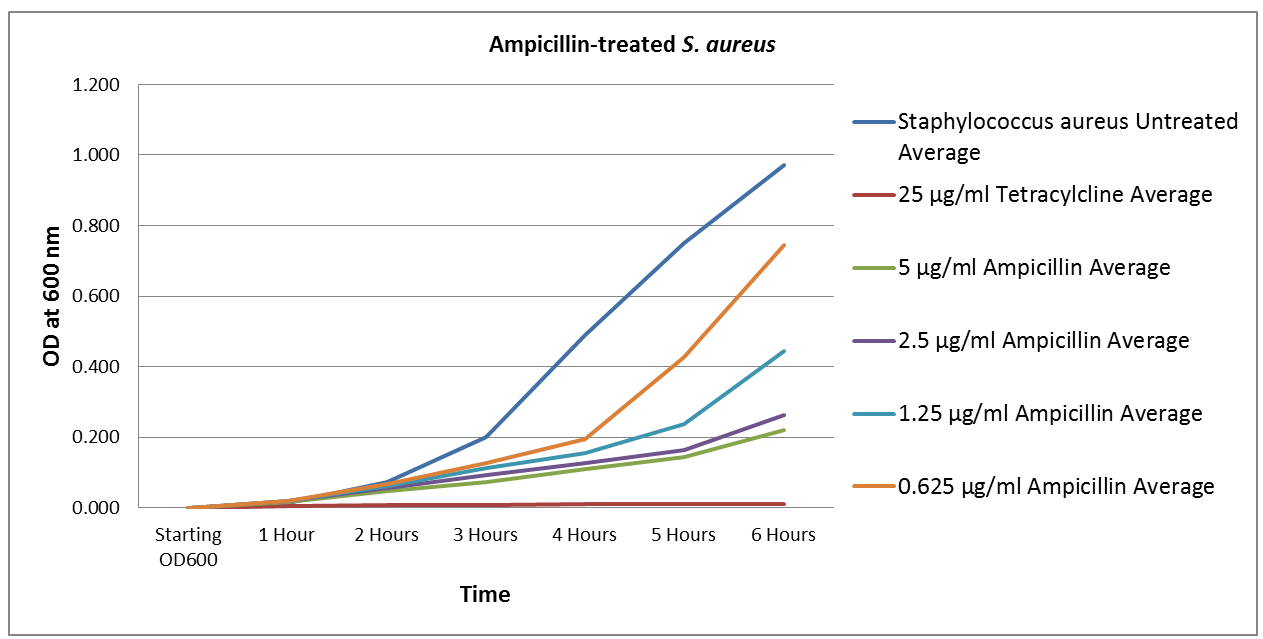

Figure 43: S. aureus treated with ampicillin

Interestingly, though also demonstrating enhanced growth inhibition, the combinatorial effects observed for the ethyl acetate extract with ampicillin were less pronounced than they were for the hexane extract with this antibiotic, despite the seemingly superior growth inhibition of the ethyl acetate extract alone (figures 44-48). At the lower ampicillin concentrations of 0.625 and $1.25 \mu \mathrm{g} / \mathrm{ml}$, improved efficacy was only significant in comparison to the antibiotic alone at these levels with extract concentrations of 250 and $500 \mu \mathrm{g} / \mathrm{ml}$.

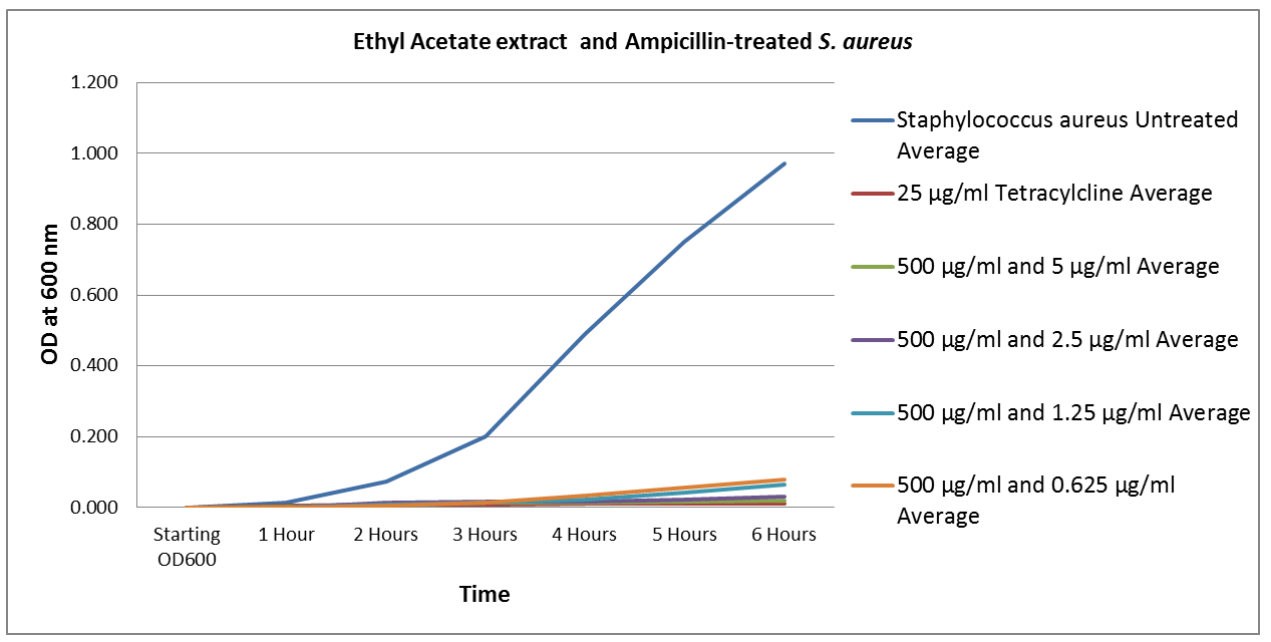

Figure 44: S. aureus treated with ampicillin and ethyl acetate extract $(500 \mu \mathrm{g} / \mathrm{ml})$ 


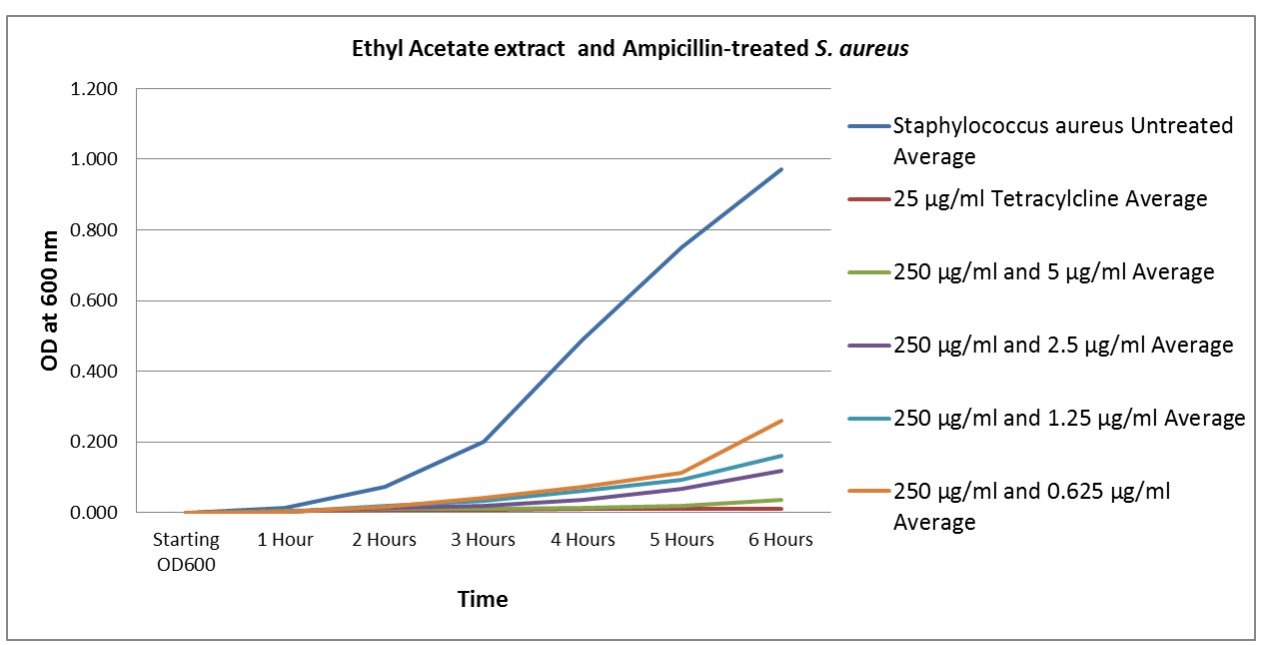

Figure 45: S. aureus treated with ampicillin and ethyl acetate extract $(250 \mu \mathrm{g} / \mathrm{ml})$

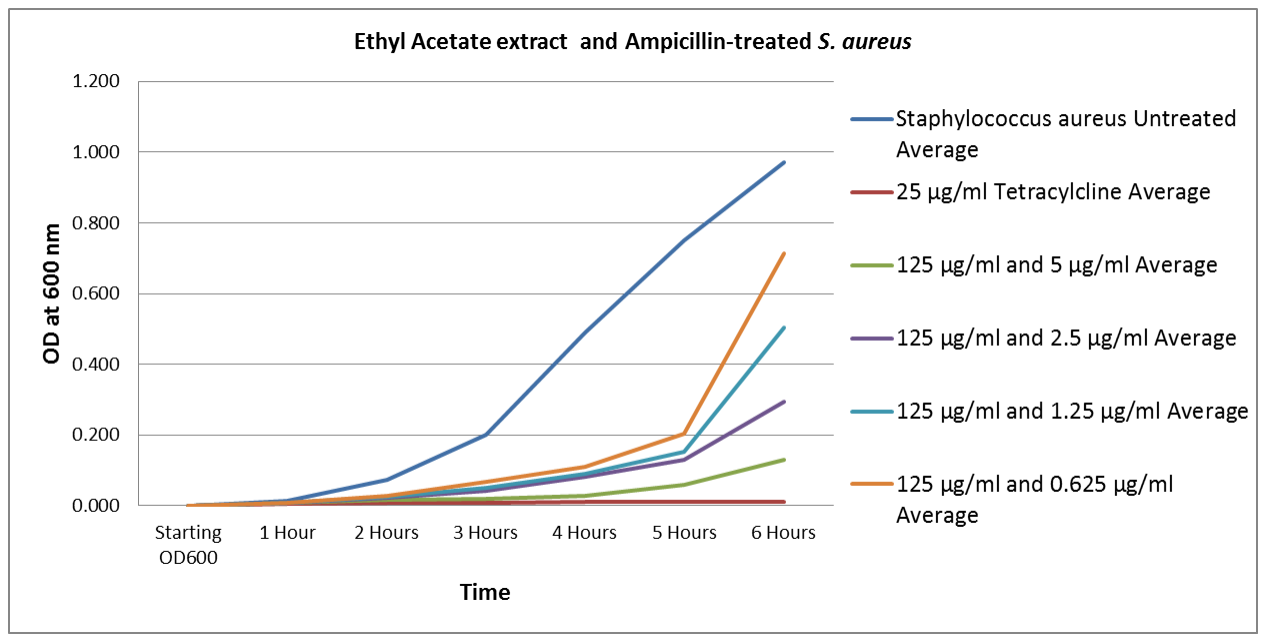

Figure 46: S. aureus treated with ampicillin and ethyl acetate extract $(125 \mu \mathrm{g} / \mathrm{ml})$ 


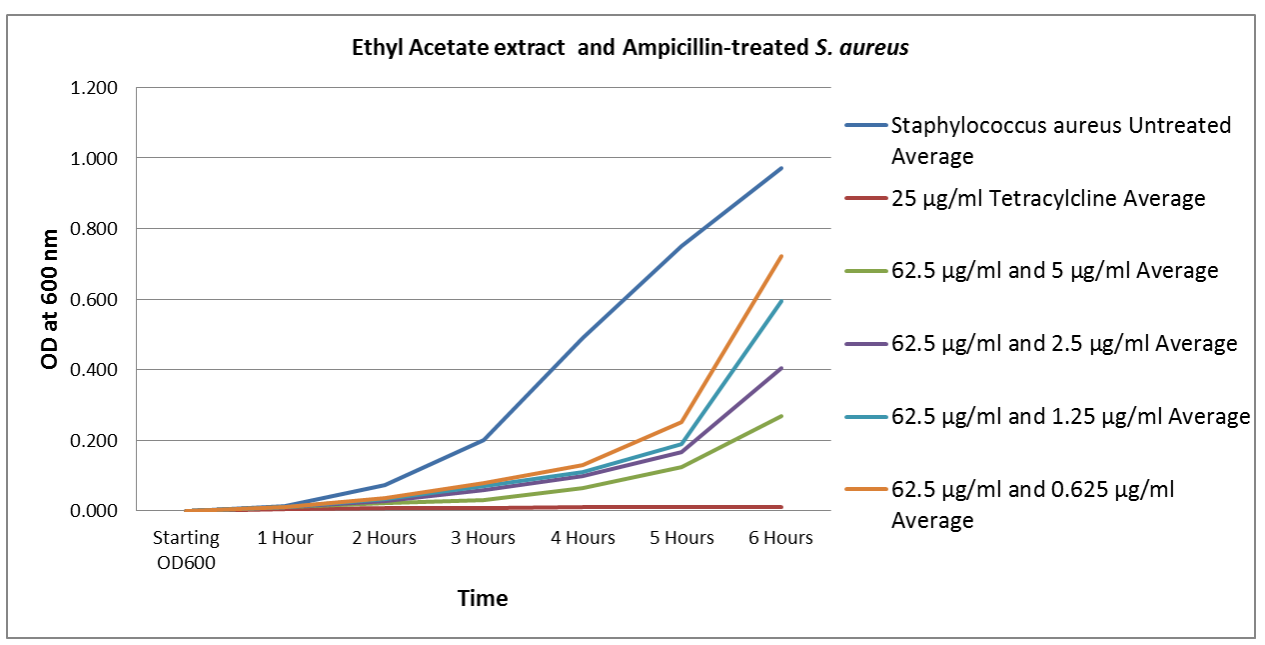

Figure 47: $S$. aureus treated with ampicillin and ethyl acetate extract $(62.5 \mu \mathrm{g} / \mathrm{ml})$

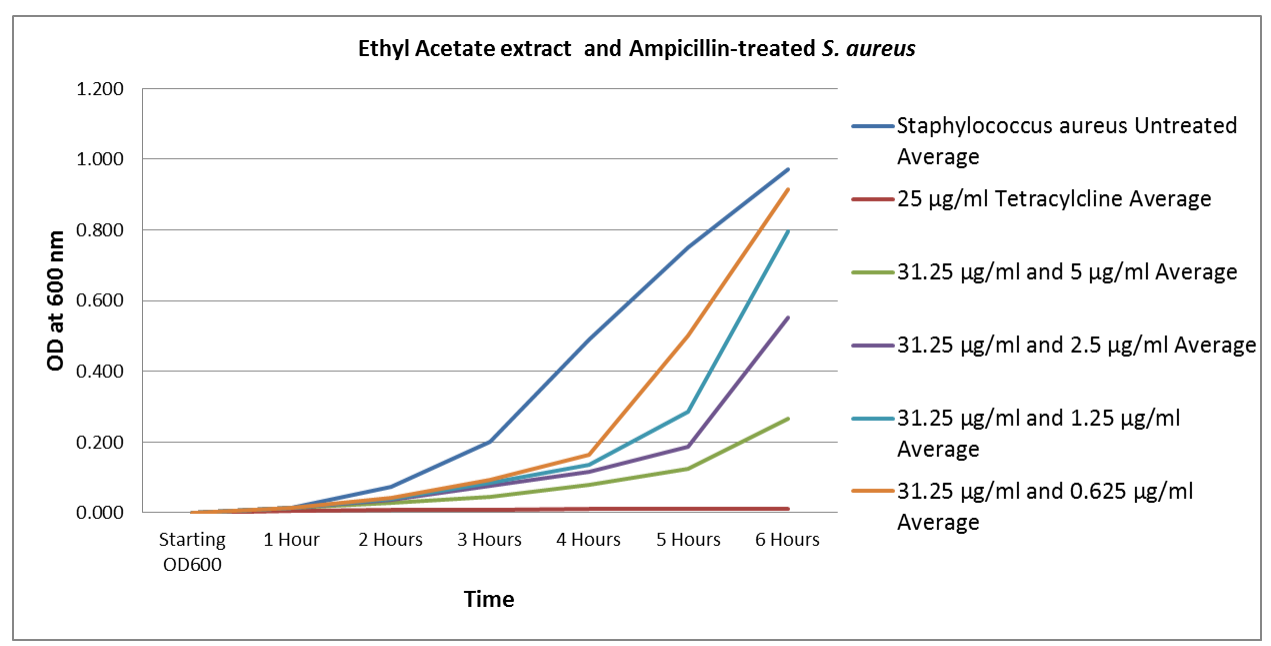

Figure 48: S. aureus treated with ampicillin and ethyl acetate extract $(31.25 \mu \mathrm{g} / \mathrm{ml})$

Finally, the antibiotics were tested alone and in combination with the water extract. As can be seen in figure 49, the water extract alone demonstrated lesser antibacterial efficacy than did the other two extracts, with significant inhibition observed only at a concentration of $500 \mu \mathrm{g} / \mathrm{ml}$. The antibiotic G418 sulfate (figure 50) alone performed in a manner similar to that observed in the preceding assays, yet with statistically significant inhibition seen only at concentrations of 5 and $10 \mu \mathrm{g} / \mathrm{ml}$. 


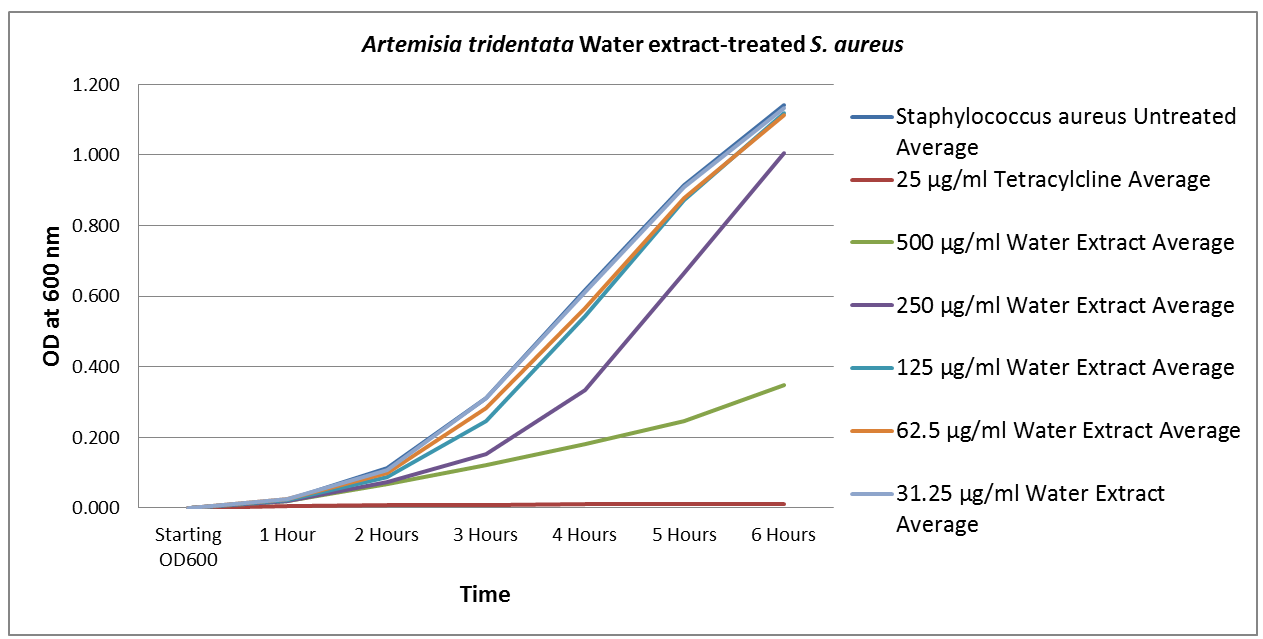

Figure 49: S. aureus treated with A. tridentata water extract

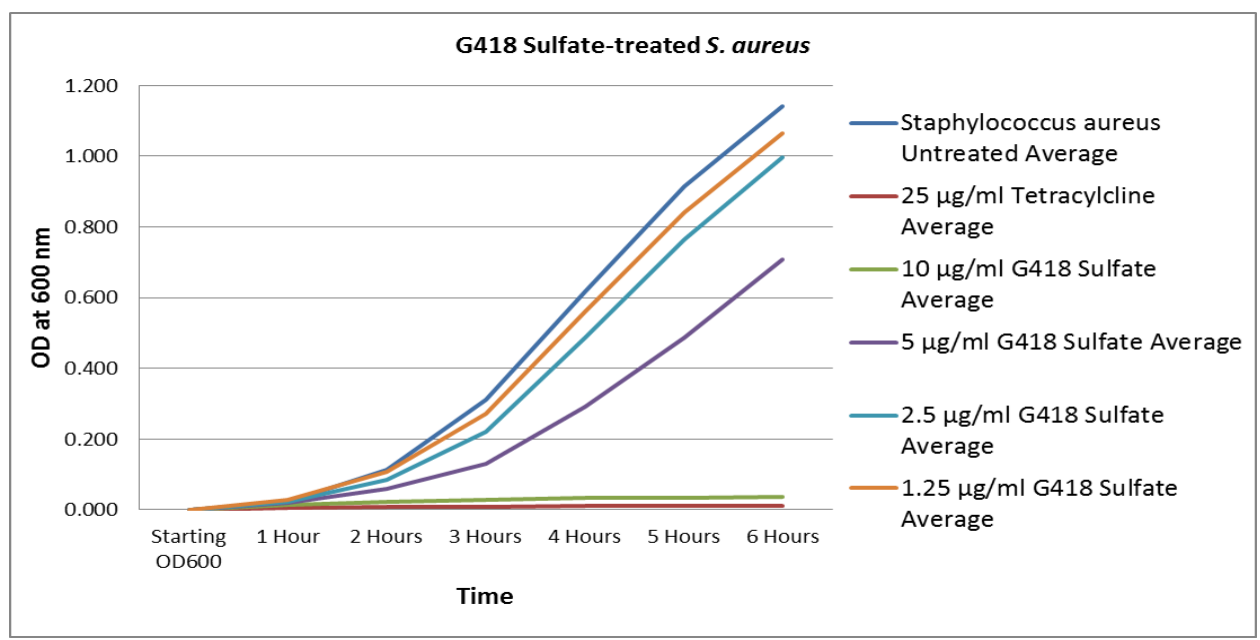

Figure 50: S. aureus treated with G418 sulfate

In combination (figures 51-55), G418 sulfate at the concentration of $1.25 \mu \mathrm{g} / \mathrm{ml}$

displayed significant growth inhibition in comparison to the untreated control only at extract levels of 250 and $500 \mu \mathrm{g} / \mathrm{ml}$, and in comparison to the antibiotic alone at this level only at an extract concentration of $500 \mu \mathrm{g} / \mathrm{ml}$. Both of these combinatorial effect comparisons were the weakest among the three extracts. 


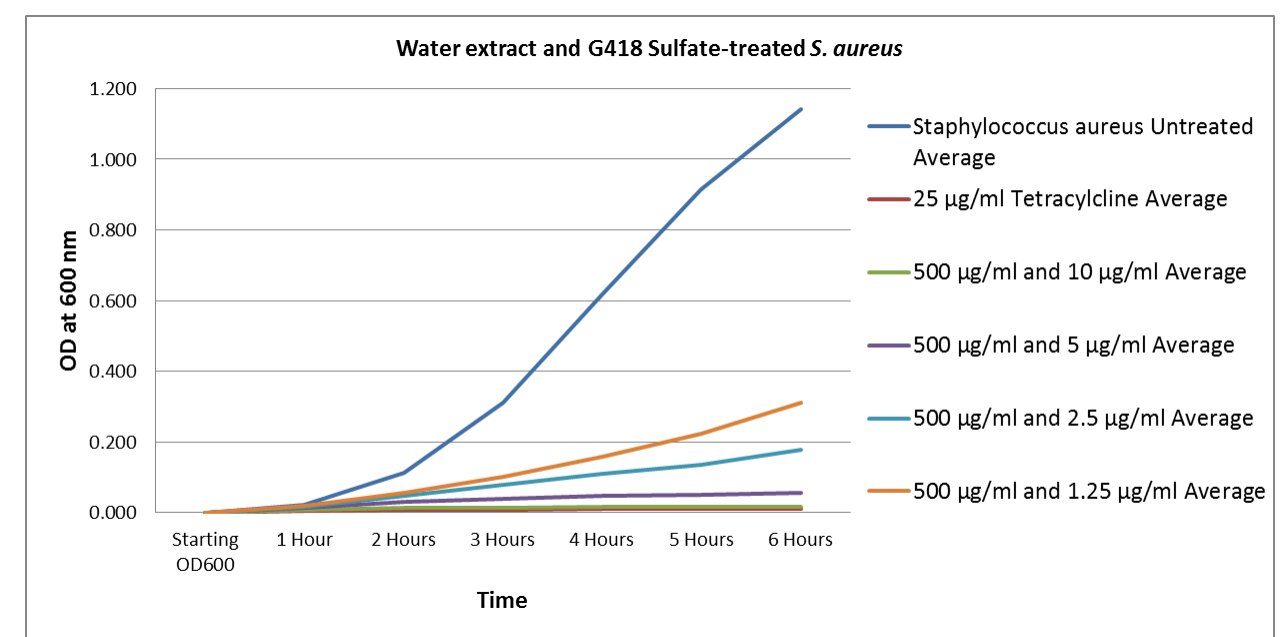

Figure 51: S. aureus treated with G418 sulfate and water extract $(500 \mu \mathrm{g} / \mathrm{ml})$

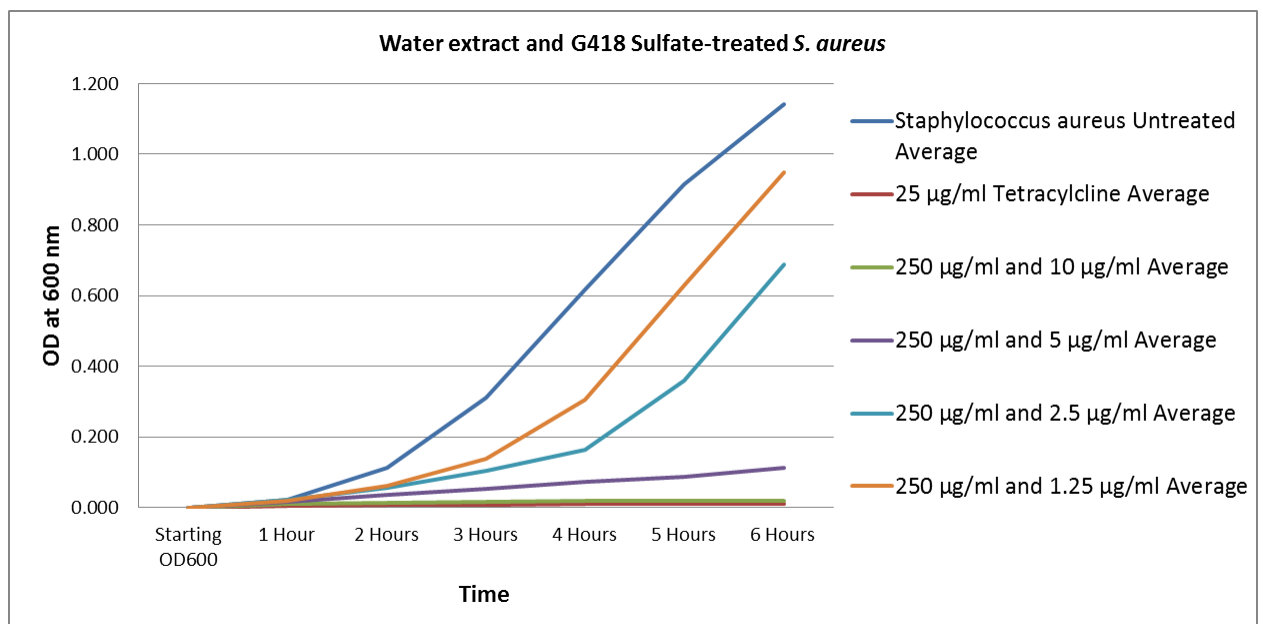

Figure 52: S. aureus treated with G418 sulfate and water extract $(250 \mu \mathrm{g} / \mathrm{ml})$ 


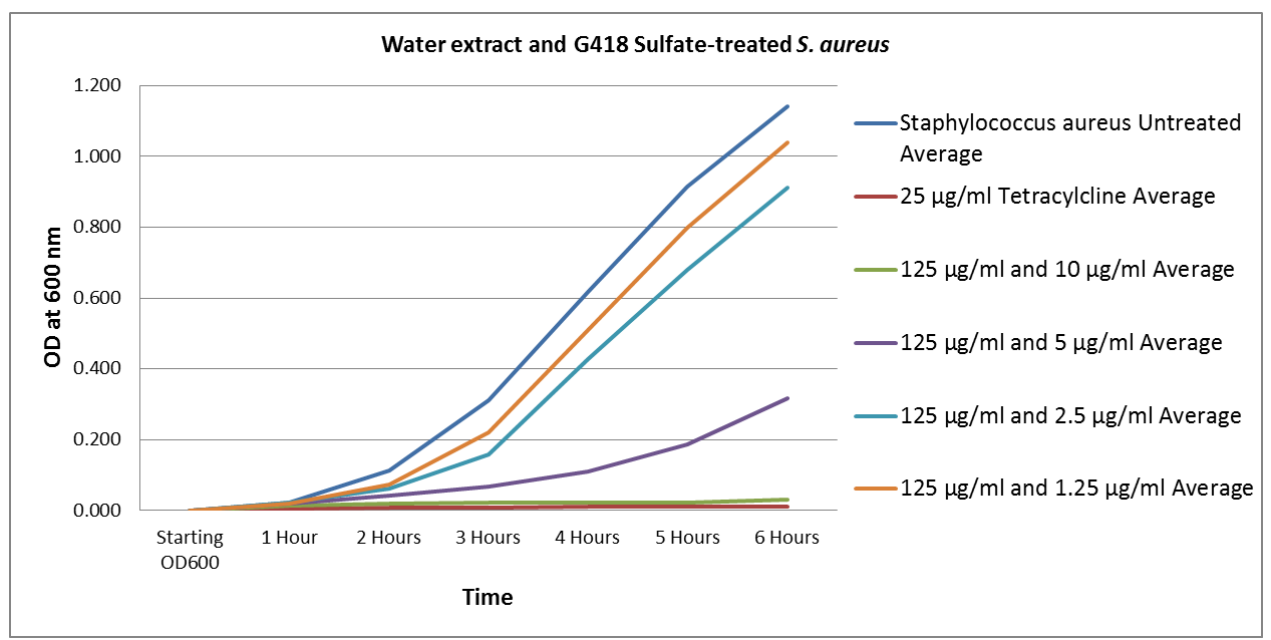

Figure 53: S. aureus treated with G418 sulfate and water extract $(125 \mu \mathrm{g} / \mathrm{ml})$

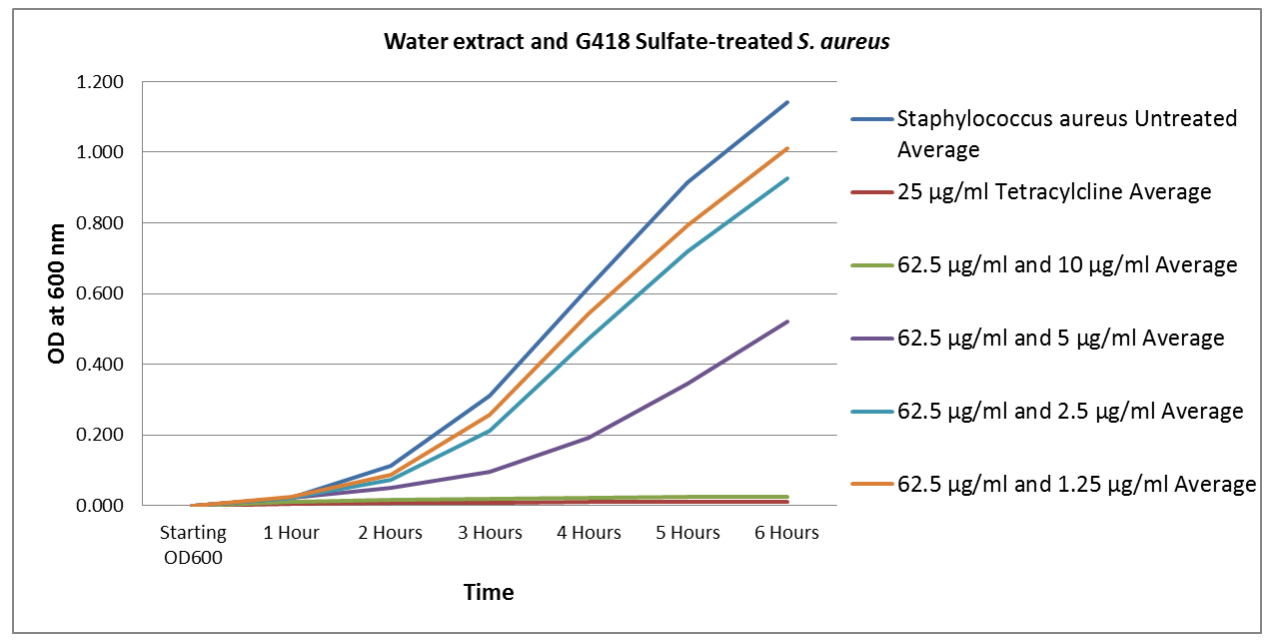

Figure 54: S. aureus treated with G418 sulfate and water extract $(62.5 \mu \mathrm{g} / \mathrm{ml})$ 


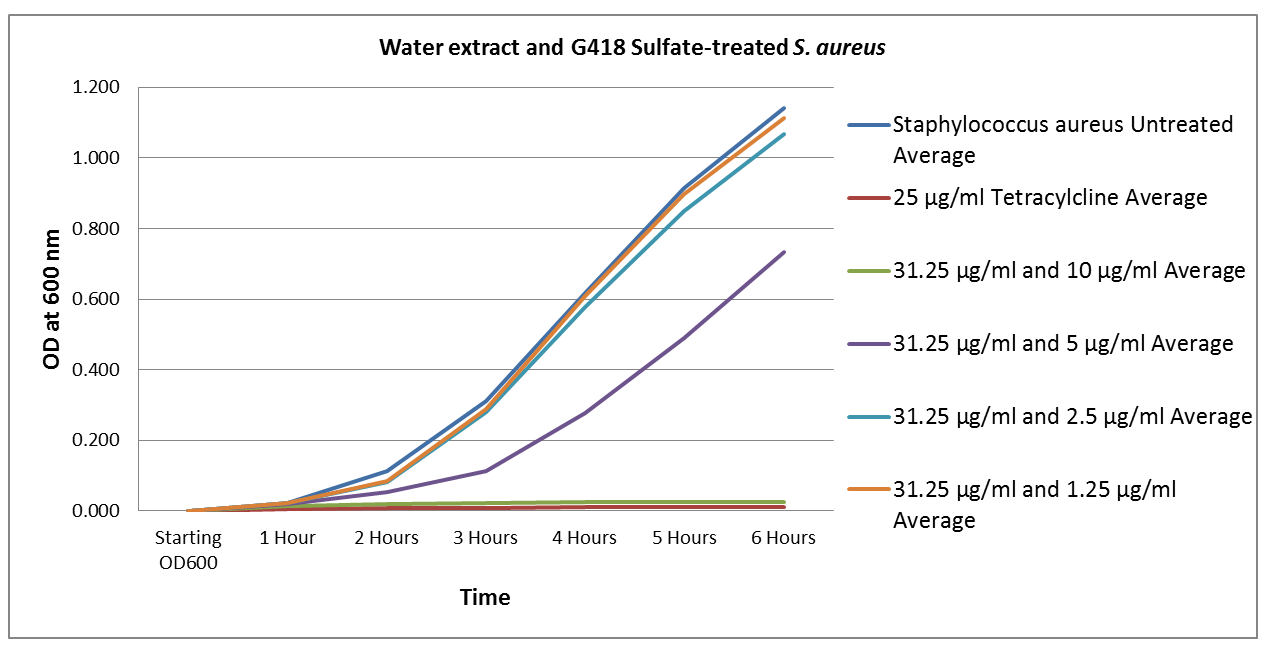

Figure 55: S. aureus treated with G418 sulfate and water extract $(31.25 \mu \mathrm{g} / \mathrm{ml})$

When tested with amikacin, the water extract alone (figure 56) performed slightly better, with significant growth inhibition seen at both 250 and $500 \mu \mathrm{g} / \mathrm{ml}$. Amikacin performance was similar to previous assays (figure 57), though in this case inhibition at the $1.25 \mu \mathrm{g} / \mathrm{ml}$ level was statistically significant.

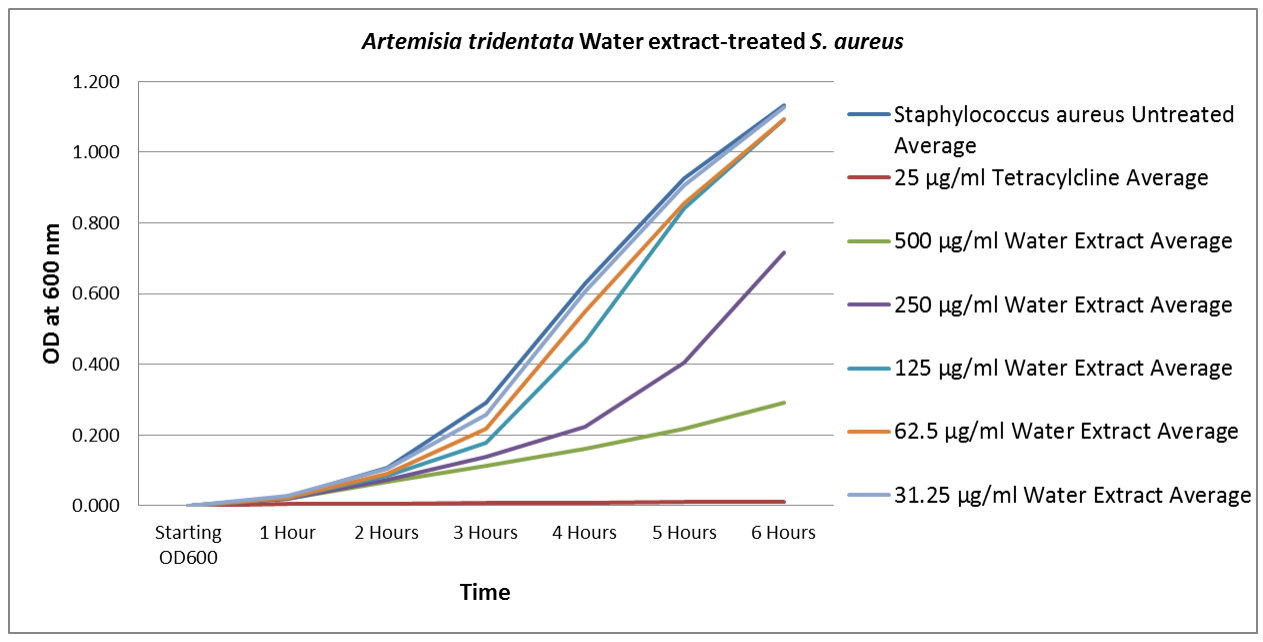

Figure 56: S. aureus treated with A. tridentata water extract 


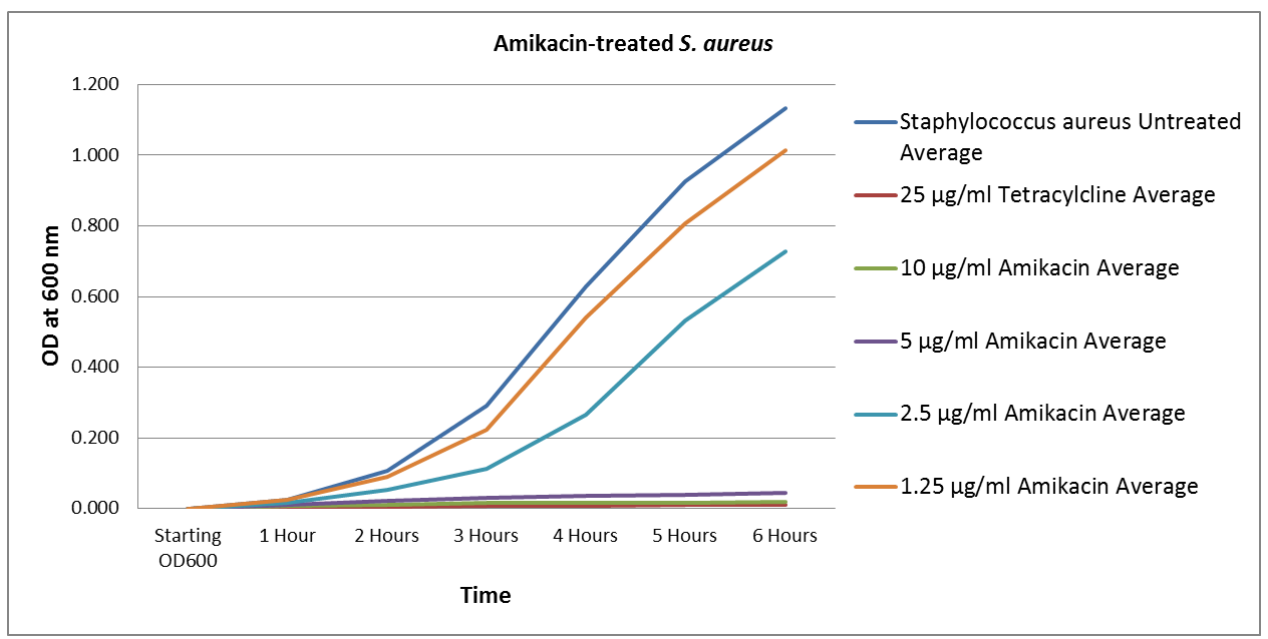

Figure 57: S. aureus treated with amikacin

In combination (figures 58-62), inhibition by amikacin at a concentration of 1.25 $\mu \mathrm{g} / \mathrm{ml}$ became significant in comparison to the antibiotic alone at this level with extract levels of $125 \mu \mathrm{g} / \mathrm{ml}$ and greater. This level of enhancement is similar to that observed for amikacin with the hexane extract, though still less than that seen when this antibiotic was combined with the ethyl acetate extract.

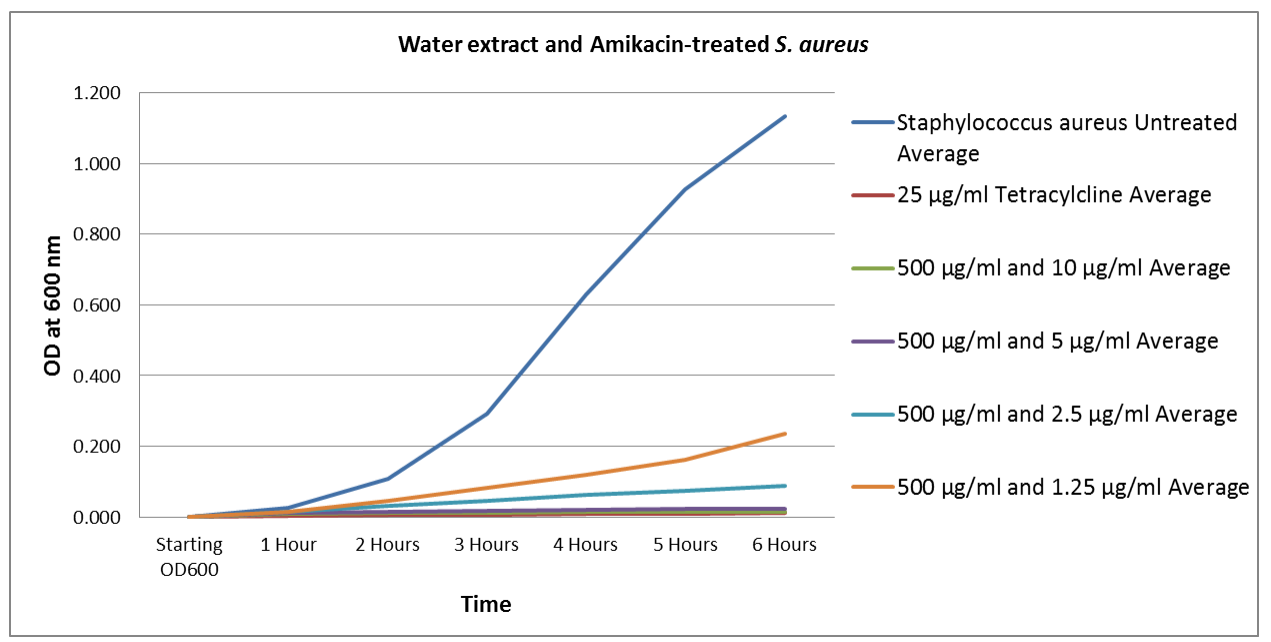

Figure 58: S. aureus treated with amikacin and water extract $(500 \mu \mathrm{g} / \mathrm{ml})$ 


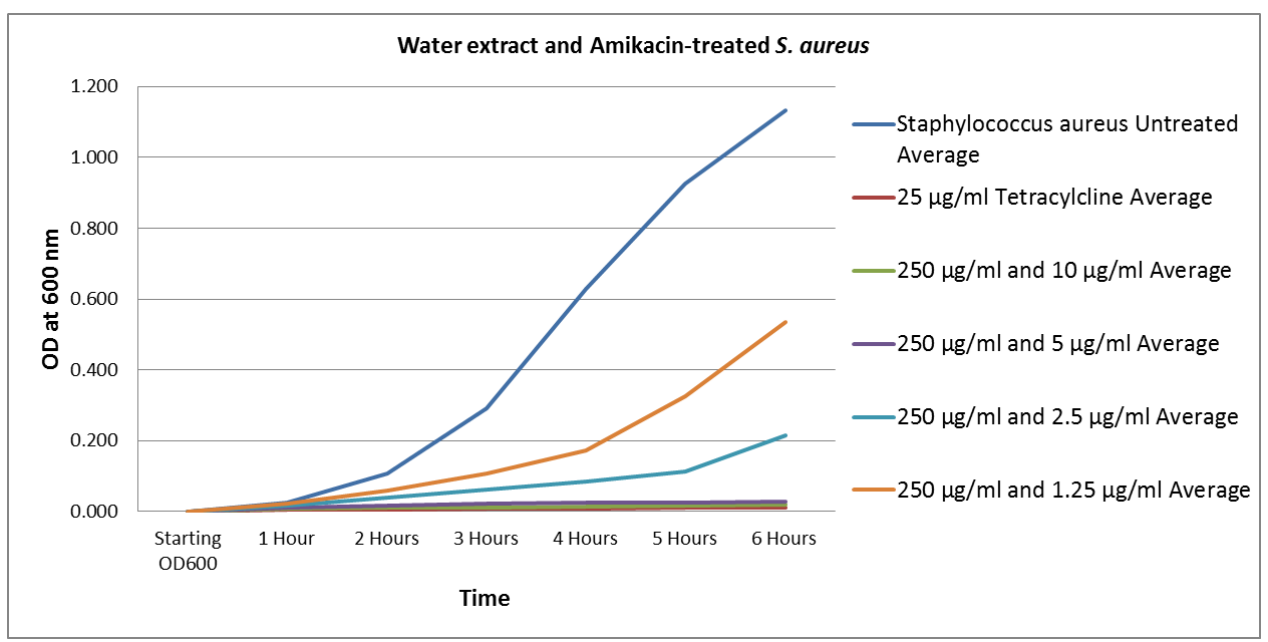

Figure 59: S. aureus treated with amikacin and water extract $(250 \mu \mathrm{g} / \mathrm{ml})$

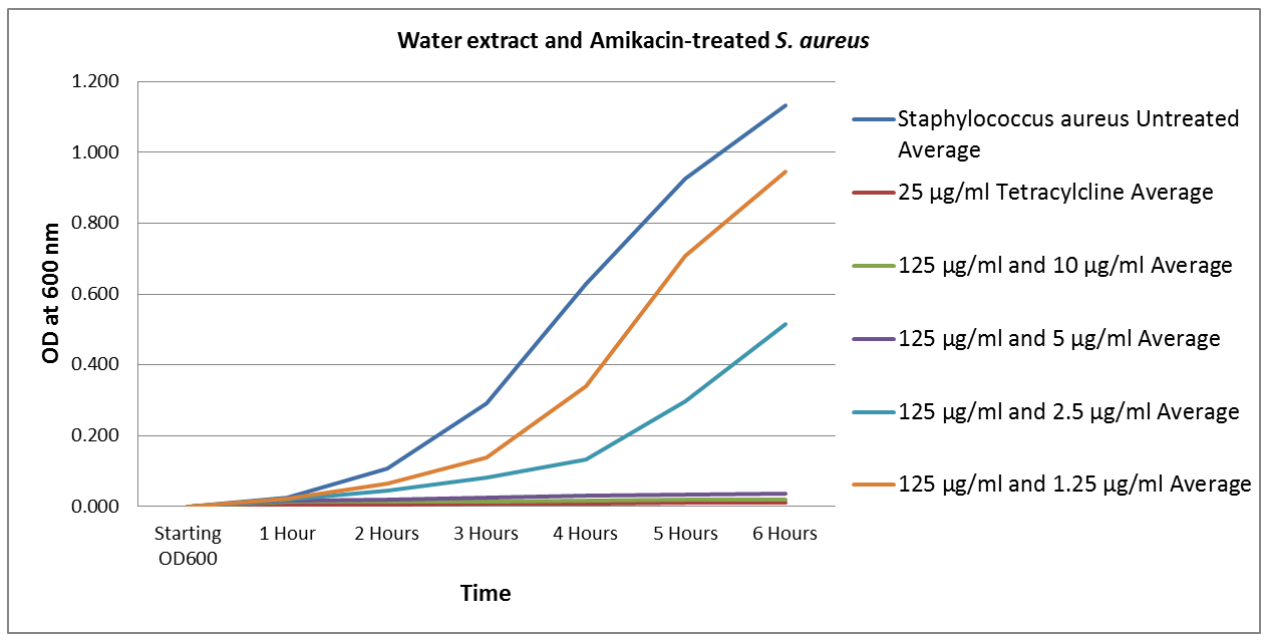

Figure 60: S. aureus treated with amikacin and water extract $(125 \mu \mathrm{g} / \mathrm{ml})$ 


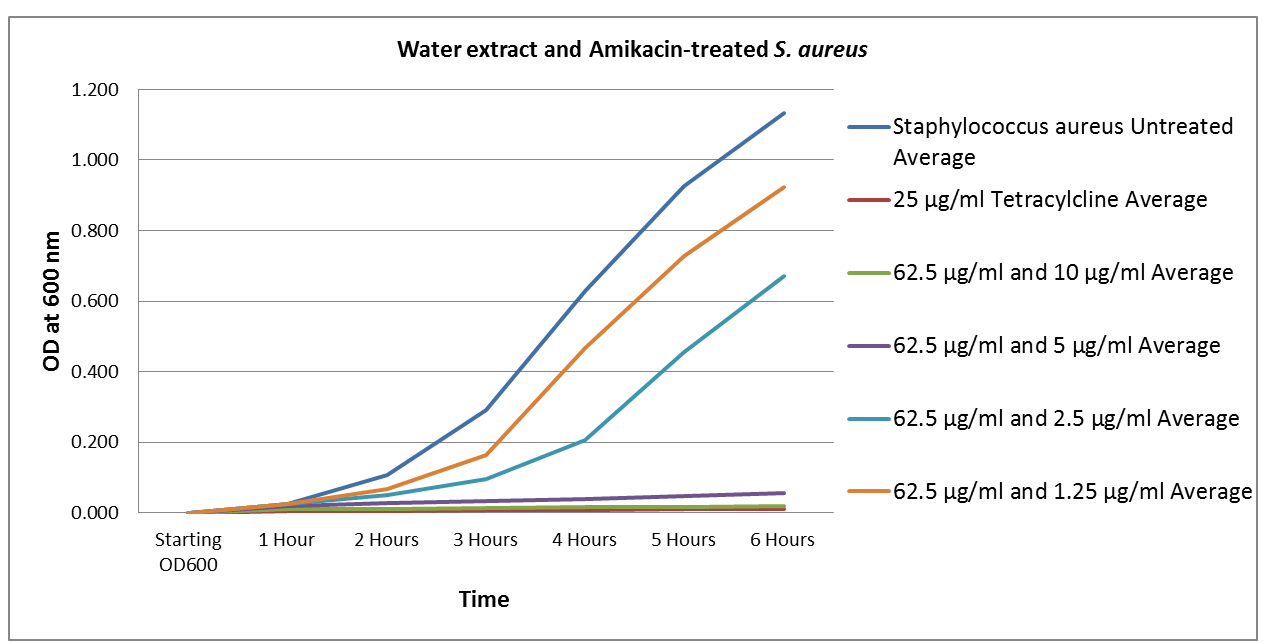

Figure 61: S. aureus treated with amikacin and water extract $(62.5 \mu \mathrm{g} / \mathrm{ml})$

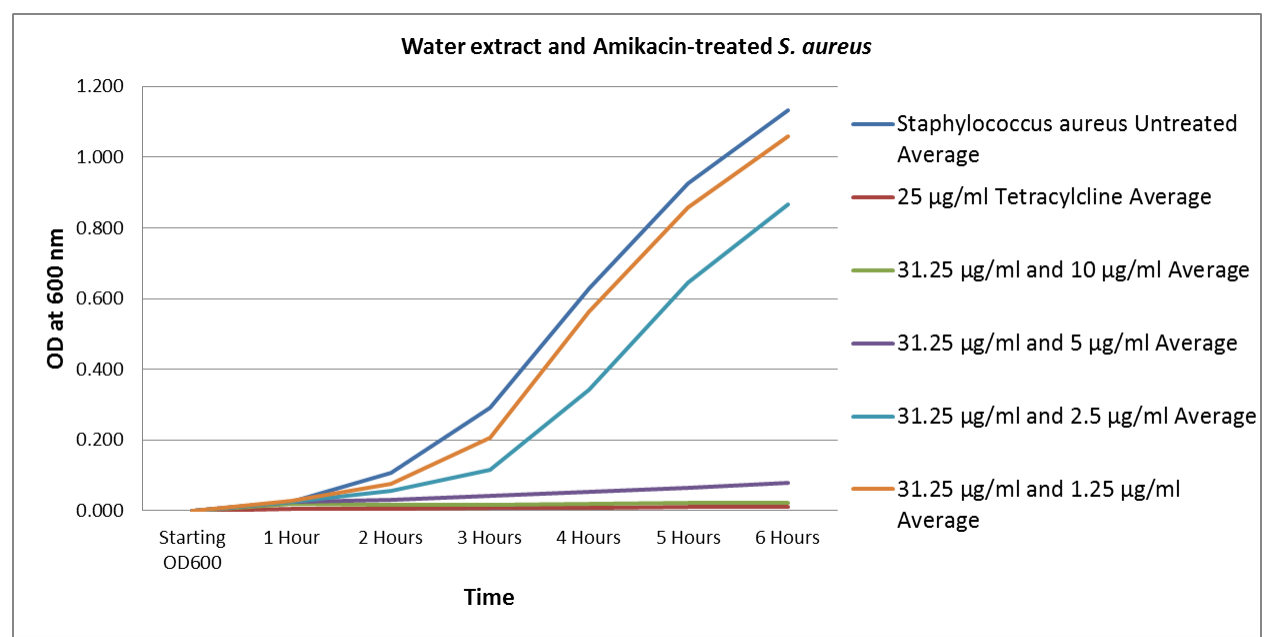

Figure 62: S. aureus treated with amikacin and water extract $(31.25 \mu \mathrm{g} / \mathrm{ml})$

Finally, when tested with ampicillin, the water extract alone (figure 63) again showed significant inhibition only at concentrations of 250 and $500 \mu \mathrm{g} / \mathrm{ml}$, while the antibiotic (figure 64) once again showed some inhibition at all concentrations tested, though in this case the effect was not significant at a concentration of $0.625 \mu \mathrm{g} / \mathrm{ml}$. 


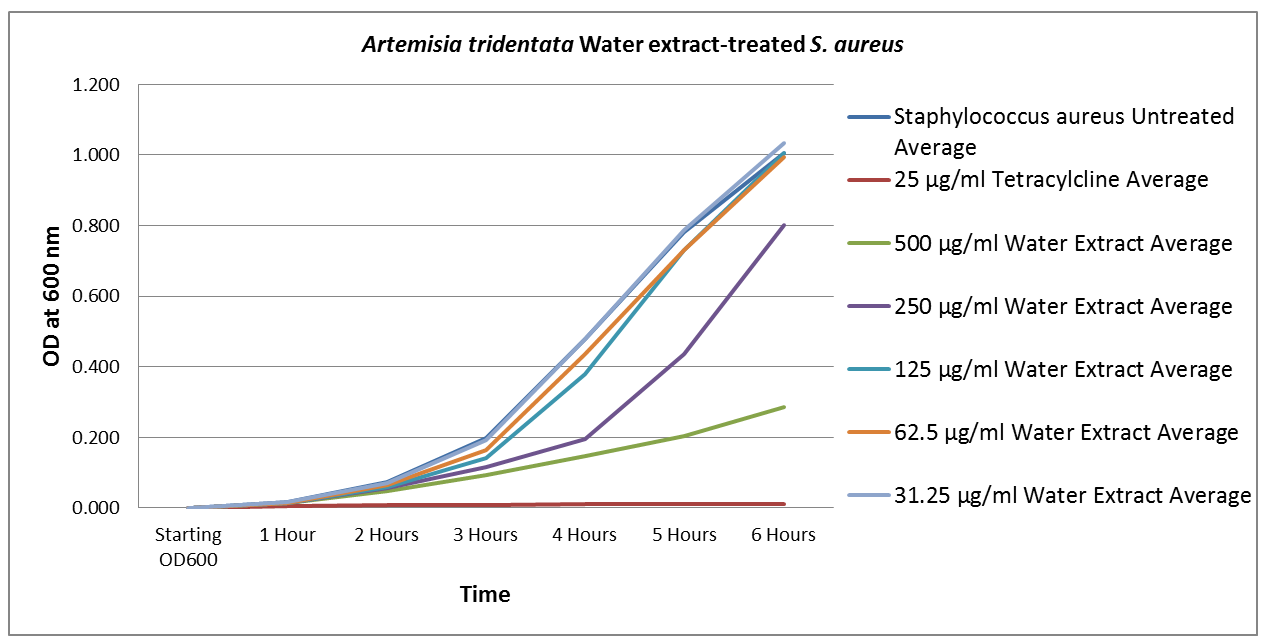

Figure 63: S. aureus treated with A. tridentata water extract

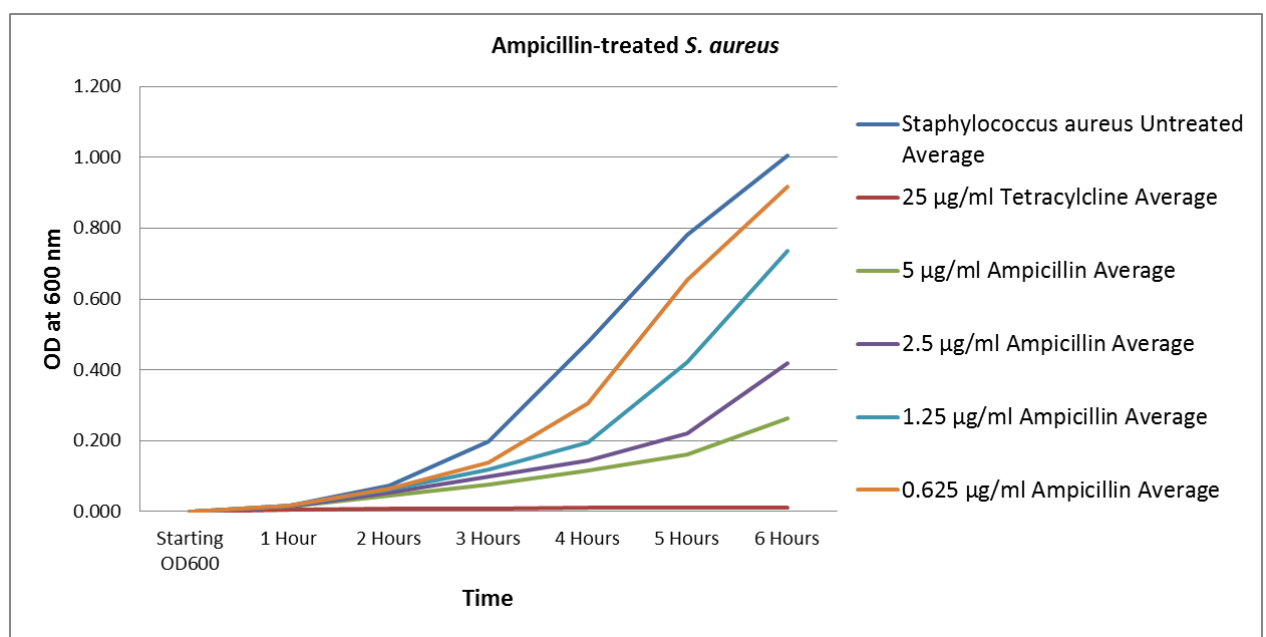

Figure 64: S. aureus treated with ampicillin

In combination (figures 65-69), inhibition by ampicillin at $0.625 \mu \mathrm{g} / \mathrm{ml}$ became significant in comparison to the untreated control with extract levels of $62.5 \mu \mathrm{g} / \mathrm{ml}$ and greater. However, improved growth inhibition by ampicillin both at this concentration and at $1.25 \mu \mathrm{g} / \mathrm{ml}$ became significant in comparison to these antibiotic concentrations alone only when combined with extract levels of 250 or $500 \mu \mathrm{g} / \mathrm{ml}$. This latter result is similar to that observed for the combination of ampicillin with the ethyl acetate extract, 
both of which were weaker than the results demonstrated by the antibiotic in combination with the hexane extract.

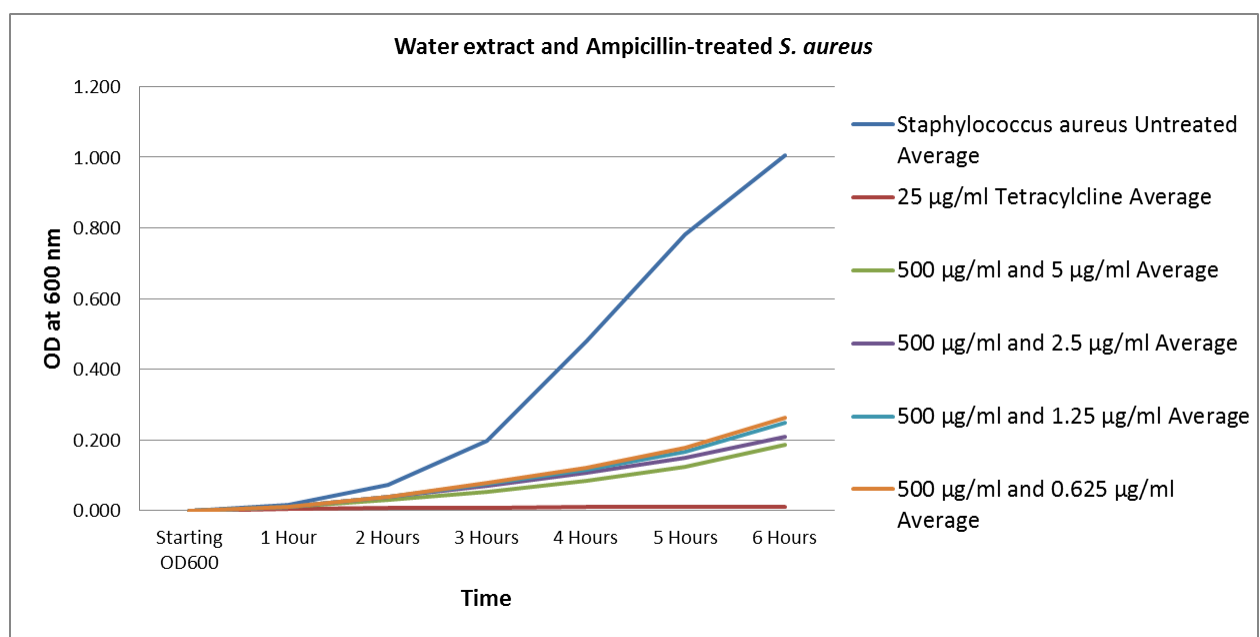

Figure 65: S. aureus treated with ampicillin and water extract $(500 \mu \mathrm{g} / \mathrm{ml})$

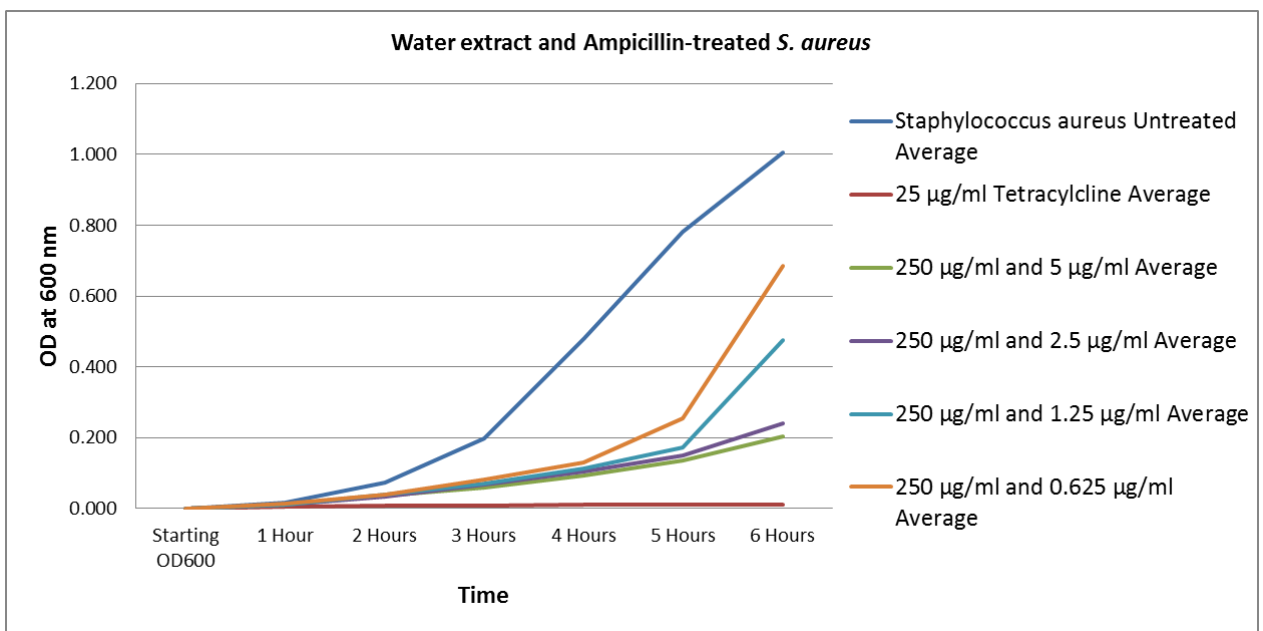

Figure 66: S. aureus treated with ampicillin and water extract $(250 \mu \mathrm{g} / \mathrm{ml})$ 


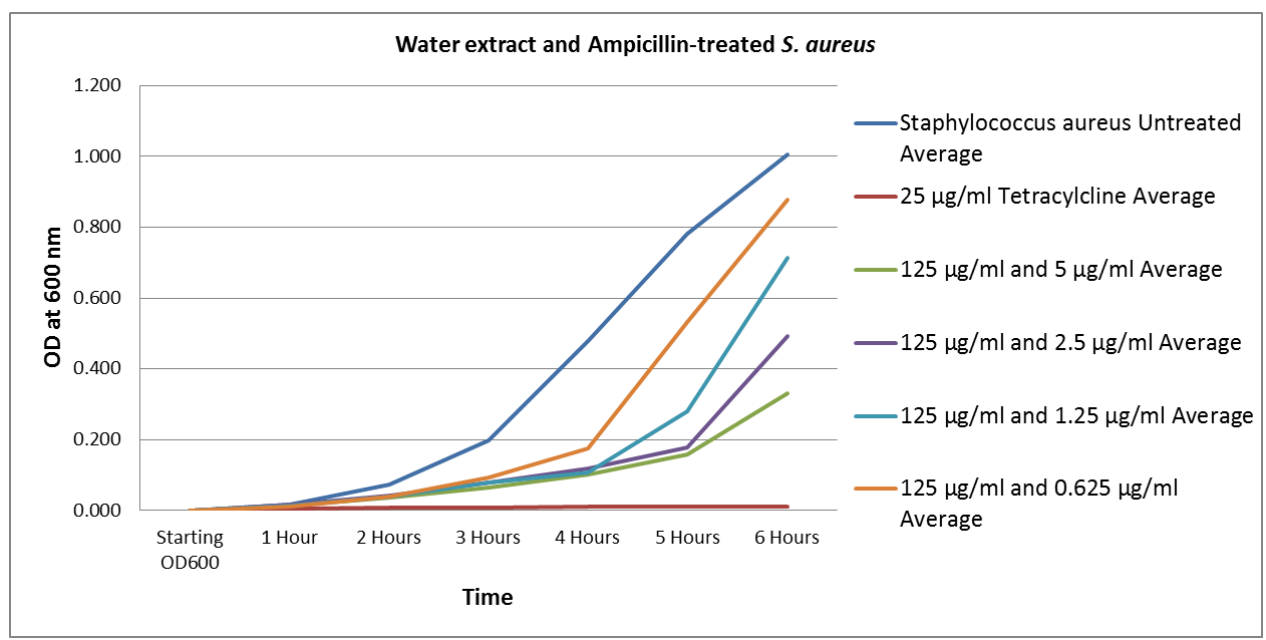

Figure 67: S. aureus treated with ampicillin and water extract $(125 \mu \mathrm{g} / \mathrm{ml})$

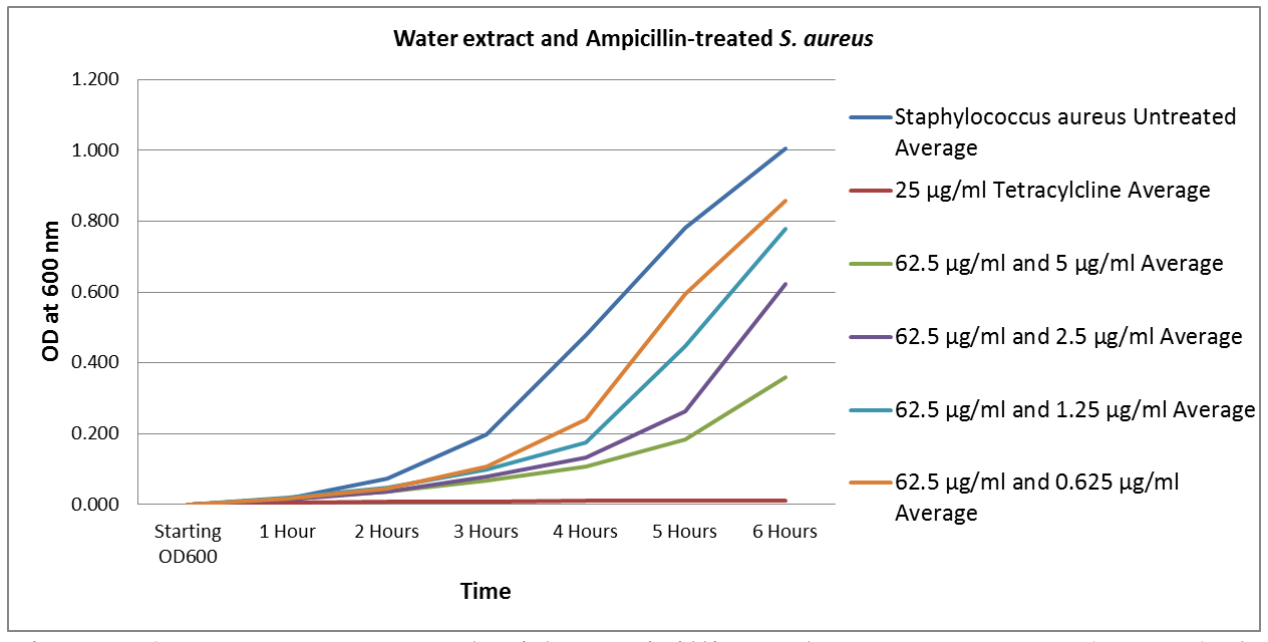

Figure 68: S. aureus treated with ampicillin and water extract $(62.5 \mu \mathrm{g} / \mathrm{ml})$ 


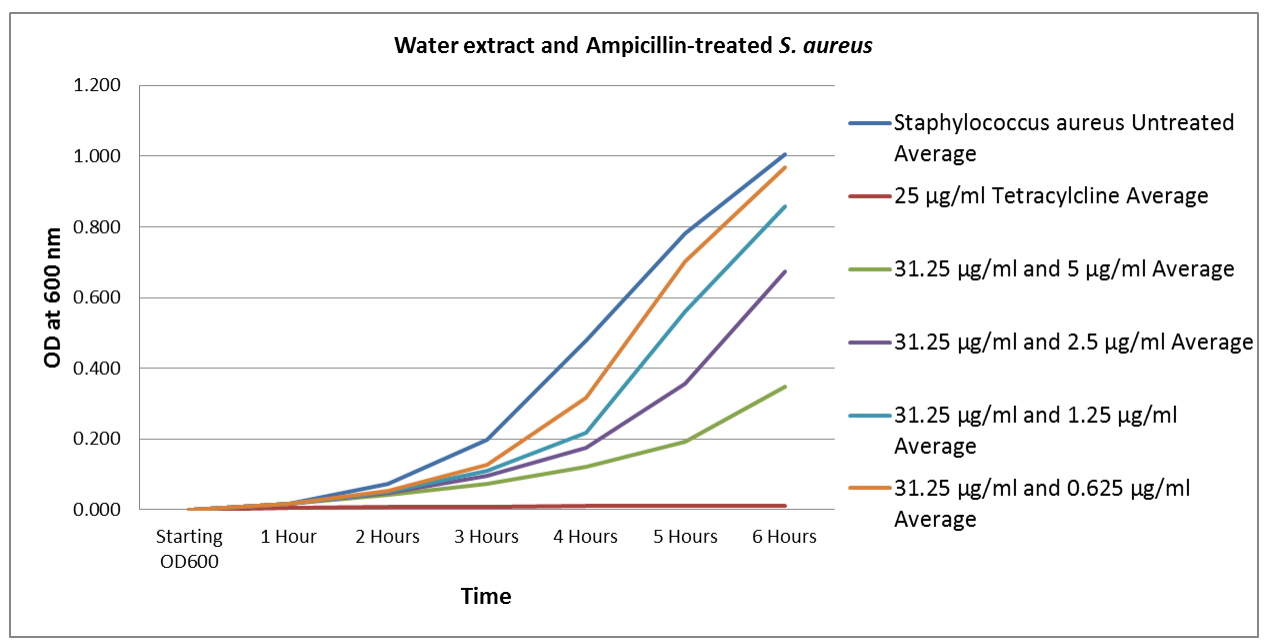

Figure 69: S. aureus treated with ampicillin and water extract $(31.25 \mu \mathrm{g} / \mathrm{ml})$ 
Time-Extended Assays

Using selected concentrations of the extracts and antibiotics, further assays were performed to approximate the length of time over which the Artemisia tridentata extracts were capable of enhancing the antibacterial efficacy of the antibiotics ampicillin, amikacin and G418 sulfate versus Staphylococcus aureus. The experimental conditions were identical to those used in the immediately above described antibacterial assays. Each of the extracts was tested alone at a concentration of $500 \mu \mathrm{g} / \mathrm{ml}$. Each of the antibiotics was tested alone at concentrations of 5 and $2.5 \mu \mathrm{g} / \mathrm{ml}$, as these levels, when used in the previous assays, demonstrated both intermediate bacterial growth inhibitory efficacy by themselves, as well as improved performance when combined with the extracts. Finally, each antibiotic at each of these two concentrations was tested in combination with each extract $(500 \mu \mathrm{g} / \mathrm{ml}) . \mathrm{OD}_{600}$ measurements were taken immediately following sample treatment and bacterial inoculation, and at hourly time points for twelve hours. Again, the averaged results from each of the three assays were then combined and averaged together. Statistical analysis was performed on the 12-hour time point values using an unpaired t-test assuming equal variances. $\mathrm{P}$ values of less than 0.05 were considered statistically significant.

As can be seen in figures 70 and 71 below, among the three extracts when tested alone, the hexane extract appears to have demonstrated the strongest antibacterial efficacy through six hours, a result consistent with previous assays at the extract concentration of $500 \mu \mathrm{g} / \mathrm{ml}$. The hexane and ethyl acetate extracts continued to display statistically significant growth inhibition through twelve hours in comparison to the untreated control, though these effects were minimal. Among the antibiotics when tested 
alone, ampicillin at both concentrations and amikacin at the concentration of $5 \mu \mathrm{g} / \mathrm{ml}$ appear to have demonstrated intermediate growth inhibition at the 6-hour time point. However, only amikacin continued to demonstrate statistically significant growth inhibition through twelve hours. The efficacy of the extracts and antibiotics in these assays were possibly lessened by more virulent growth of the bacteria, as the untreated controls demonstrated slightly greater $\mathrm{OD}_{600}$ levels after six hours in comparison to previous assays.

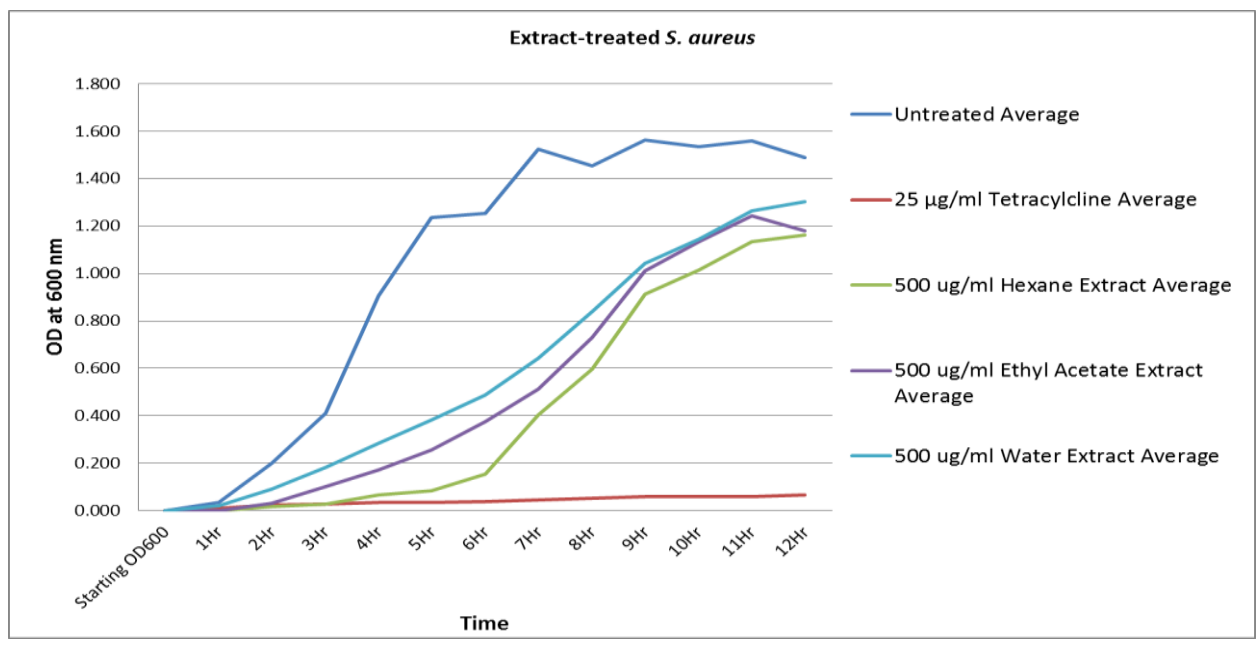

Figure 70: S. aureus treated with A. tridentata extracts 


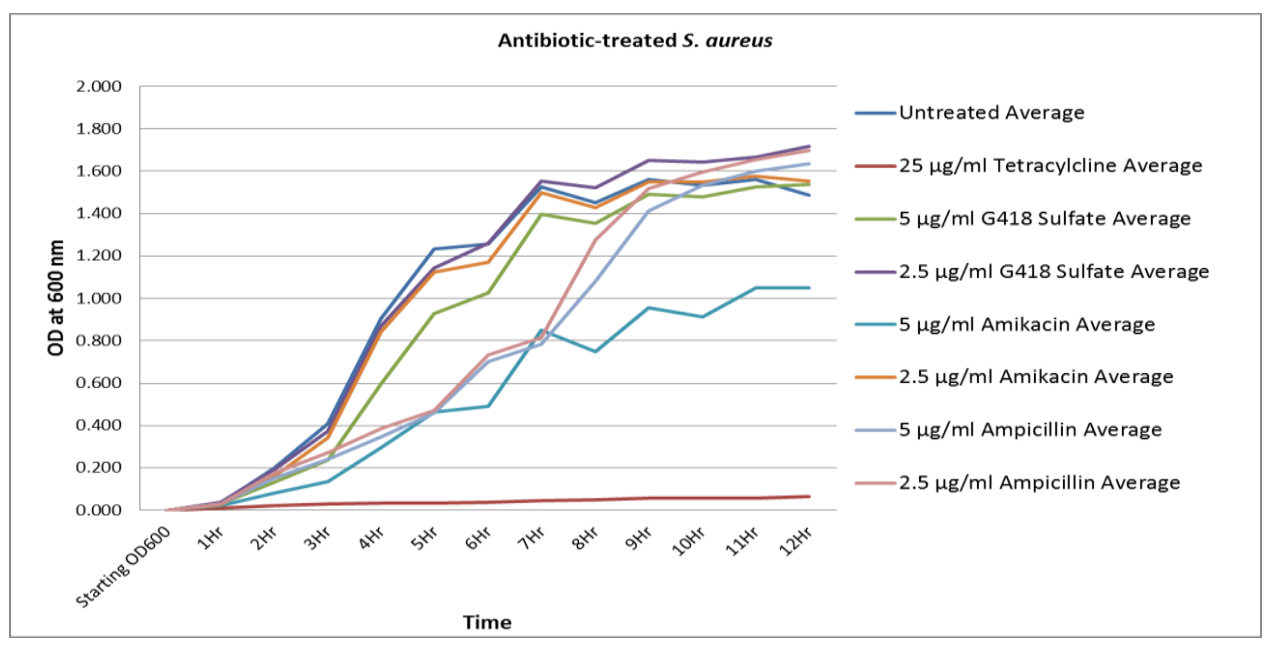

Figure 71: S. aureus treated with G418 sulfate, amikacin, ampicillin

Still, in combination, as can be seen in figures $72-74$, antibacterial efficacy was again improved. G418 sulfate growth inhibition at the 12-hour time point became statistically significant in comparison to the untreated control when combined with each extract, with the growth curves of samples treated with G418 sulfate and either the hexane or ethyl acetate extracts resembling that of the positive control. Enhancement of growth inhibition by all extracts was also statistically significant in comparison to the antibiotic alone at both concentrations.

Similarly, the growth curves of samples treated with amikacin at $5 \mu \mathrm{g} / \mathrm{ml}$, in combination with either the hexane or ethyl acetate extracts, resembled that of the positive control, with the enhancement of growth inhibition statistically significant in comparison to the antibiotic alone at this concentration. However, the water extract significantly reduced the efficacy of amikacin at $5 \mu \mathrm{g} / \mathrm{ml}$. At $2.5 \mu \mathrm{g} / \mathrm{ml}$, growth inhibition by amikacin became significant in comparison to the untreated control when combined with the hexane or ethyl acetate extracts, but not the water extract, again with 
the growth curves of samples treated with combinations of amikacin and the hexane or ethyl acetate extracts resembling that of the positive control. While the enhancement of growth inhibition by the combination of amikacin at $2.5 \mu \mathrm{g} / \mathrm{ml}$ with all of the extracts was statistically significant in comparison to the antibiotic alone at this concentration, it can be seen that the effect of the water extract was minimal.

Extract enhancement of growth inhibition by ampicillin was less pronounced. At $5 \mu \mathrm{g} / \mathrm{ml}$, growth inhibition in comparison to the untreated control only became statistically significant when ampicillin was combined with the hexane extract. Though enhancement of growth inhibition by all extracts was statistically significant in comparison to ampicillin alone at this concentration, this is attributable to the high final $\mathrm{OD}_{600}$ level of the ampicillin-treated sample, and it can be seen from the figure that only the hexane extract noticeably improved antibiotic efficacy. At $2.5 \mu \mathrm{g} / \mathrm{ml}$, similar results were observed, with only the hexane extract significantly improving the efficacy of amikacin in comparison to the untreated control, or the antibiotic alone at this concentration. 


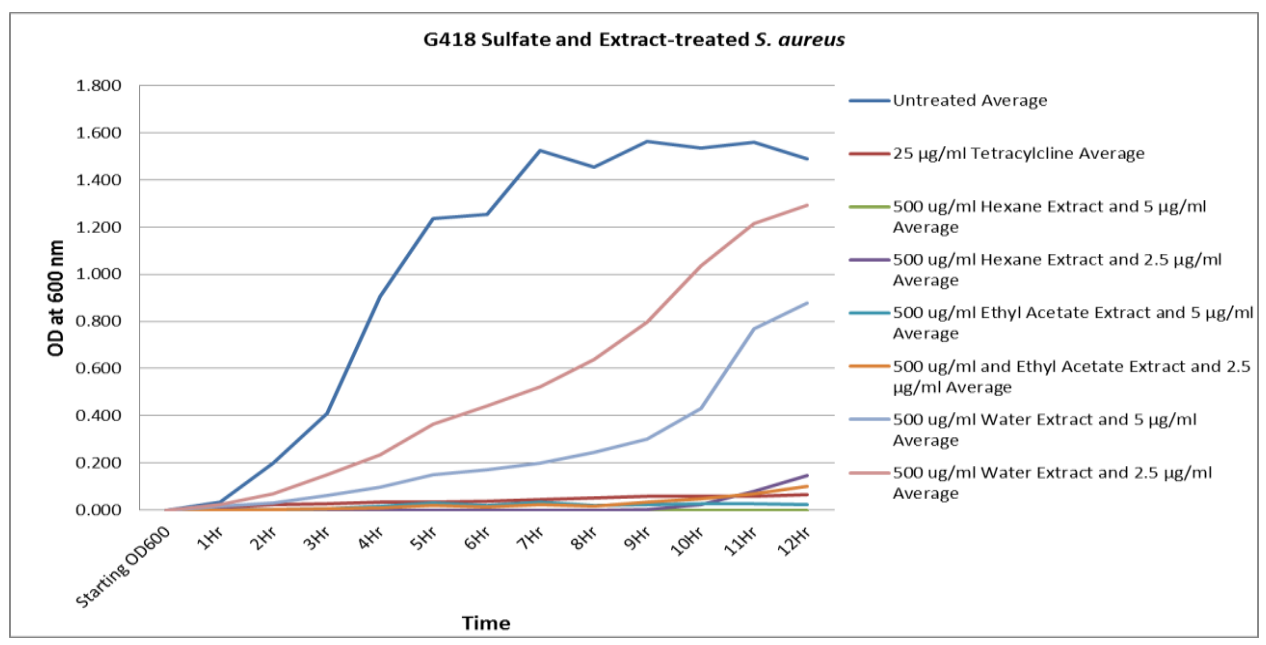

Figure 72: S. aureus treated with G418 sulfate and A. tridentata extracts

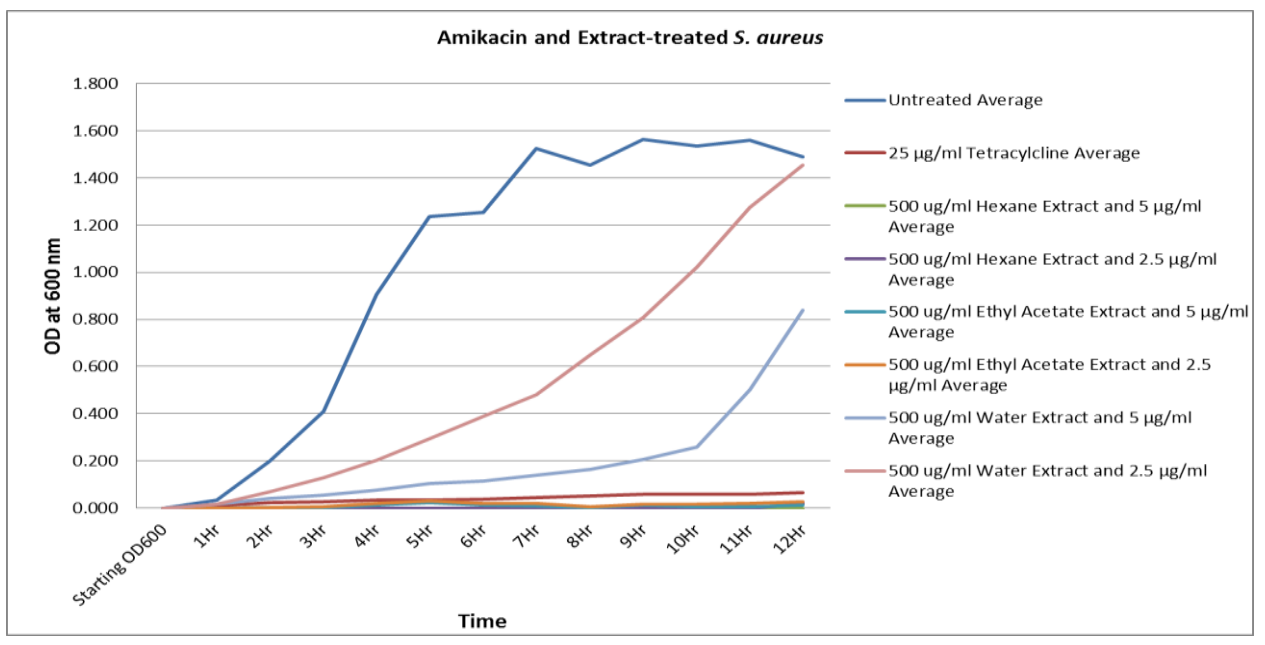

Figure 73: S. aureus treated with amikacin and A. tridentata extracts 


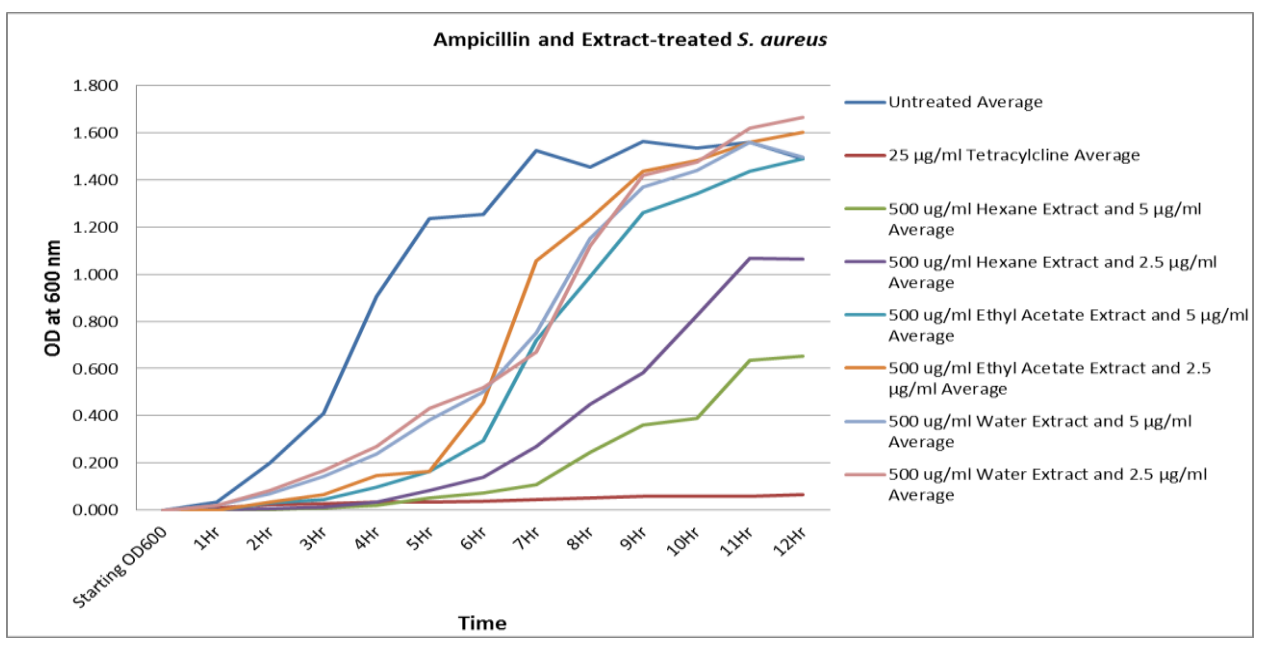

Figure 74: S. aureus treated with ampicillin and A. tridentata extracts 


\section{Extracts in Combination}

The extracts were also assayed in combination with each other to observe for any negating or enhancement of antibacterial efficacy which might indicate the presence of antagonistic, additive or synergistic compounds present in the original plant material. Experimental conditions were the same as those described above. Each extract was tested alone at concentrations of 500, 250, 125 and $62.5 \mu \mathrm{g} / \mathrm{ml}$, and in combination at concentrations of $250,125,62.5$ and $31.25 \mu \mathrm{g} / \mathrm{ml}$.

As can be seen in figures 75-77, the performances of the extracts alone were similar to those observed in previous assays, with statistically significant growth inhibition observed at all hexane and ethyl acetate extract concentrations, and at water extract concentrations of 500 and $250 \mu \mathrm{g} / \mathrm{ml}$.

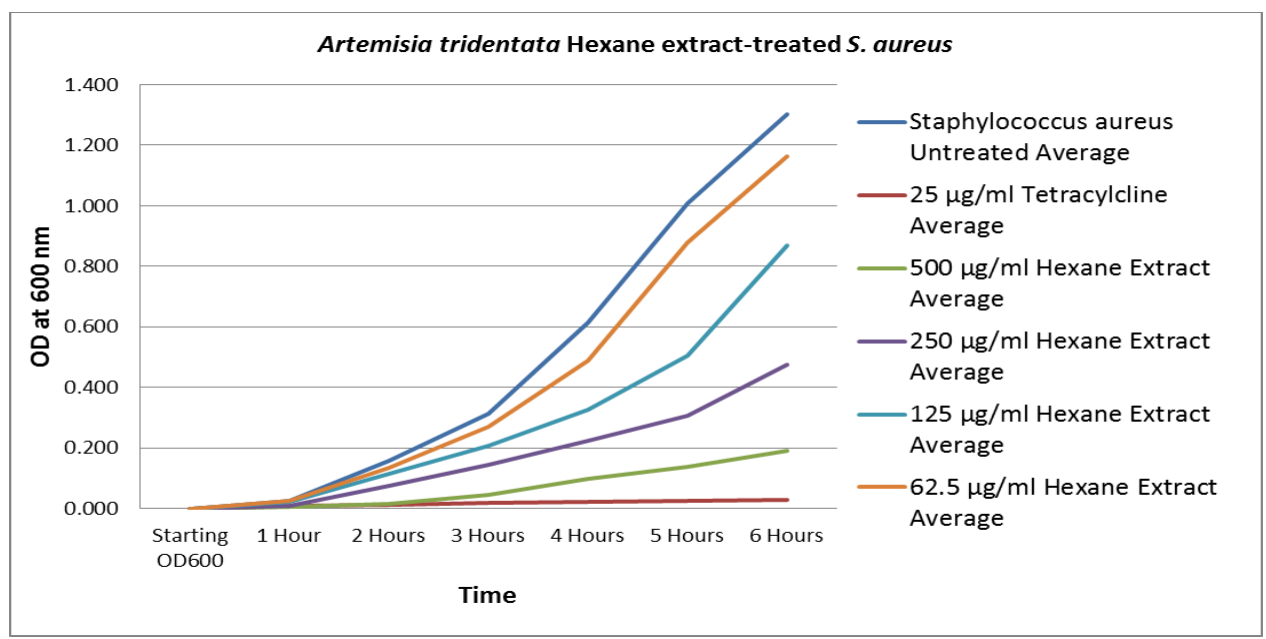

Figure 75: S. aureus treated with A. tridentata hexane extract 


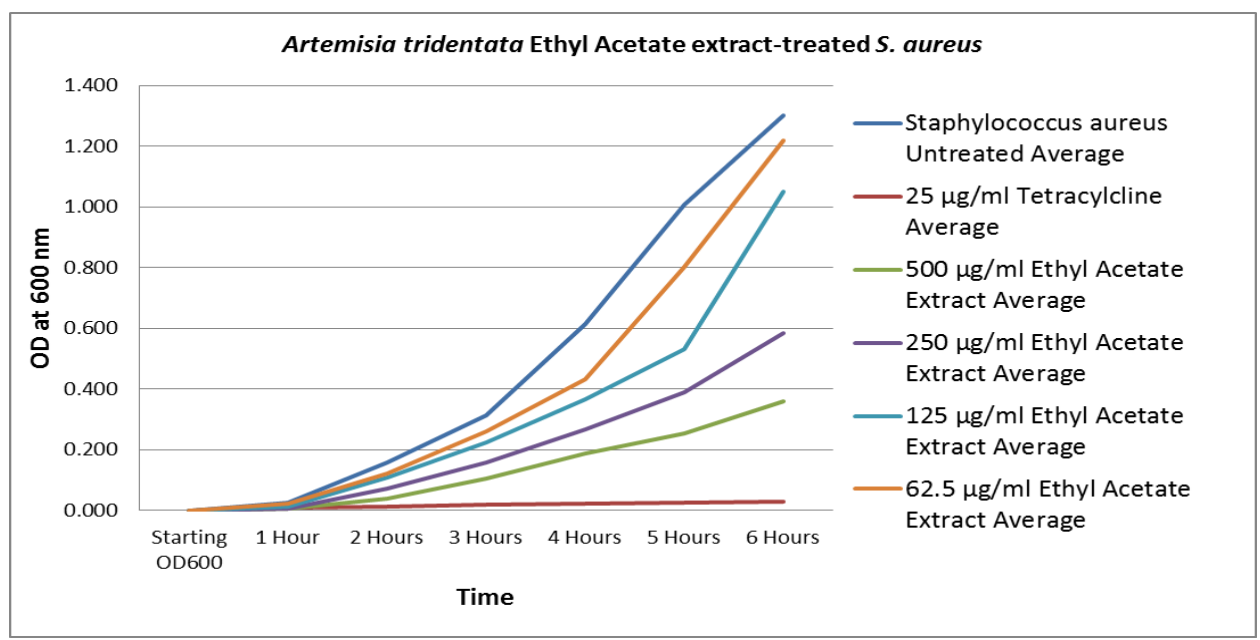

Figure 76: S. aureus treated with A. tridentata ethyl acetate extract

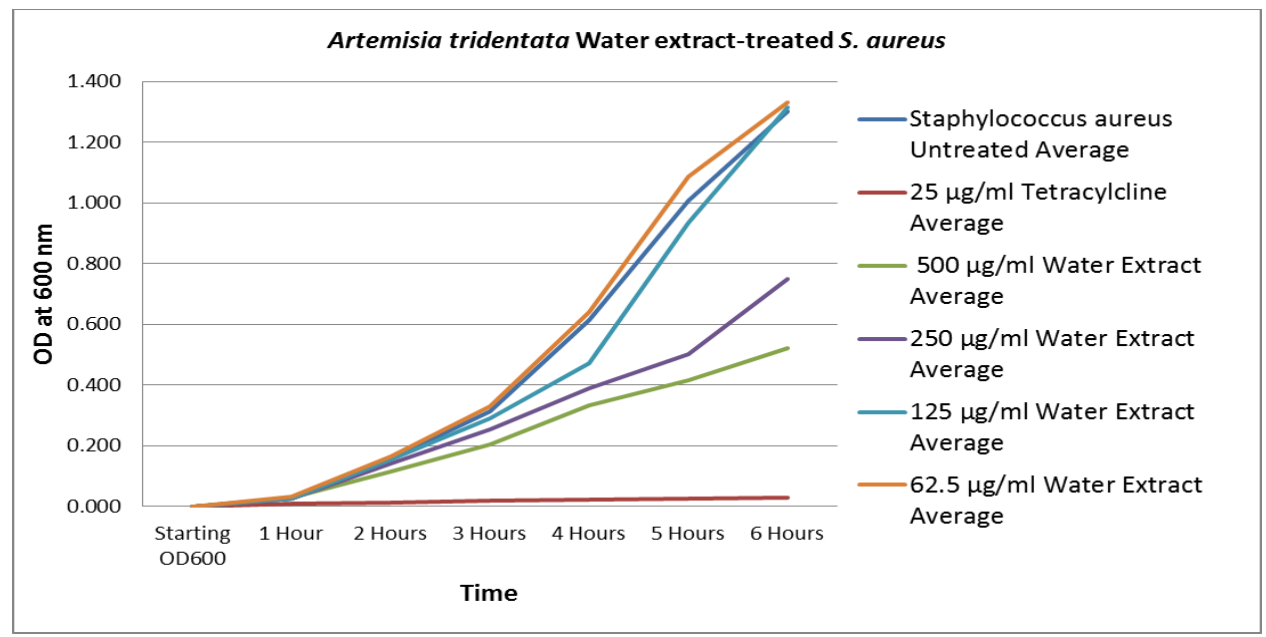

Figure 77: S. aureus treated with A. tridentata water extract

Using the same method of statistical analysis, the $\mathrm{OD}_{600}$ values measured at the 6hour time point for each pairing of extracts was compared to the average of the values of each extract alone at the total extract concentration. For example, the final reading for the combination of the ethyl acetate and hexane extracts (figure 78) at $250 \mu \mathrm{g} / \mathrm{ml}$ each 
was compared to the average between the final readings for the ethyl acetate and hexane extracts alone when tested at $500 \mu \mathrm{g} / \mathrm{ml}$. Though the dose-response relationships between the extracts and growth inhibition in these or previous assays do not appear to have demonstrated perfectly linear correlations, it would be reasonable to expect that dramatic antagonistic, additive or synergistic effects between extracts might result in observable effects on the growth curves of treated samples. In the case of the hexane and ethyl acetate extracts, this combination showed significant inhibition versus the untreated control only at concentrations of 125 and $250 \mu \mathrm{g} / \mathrm{ml}$ each, with statistically significant diminishment of antibacterial efficacy in comparison to the averages of the extracts alone at all concentrations tested.

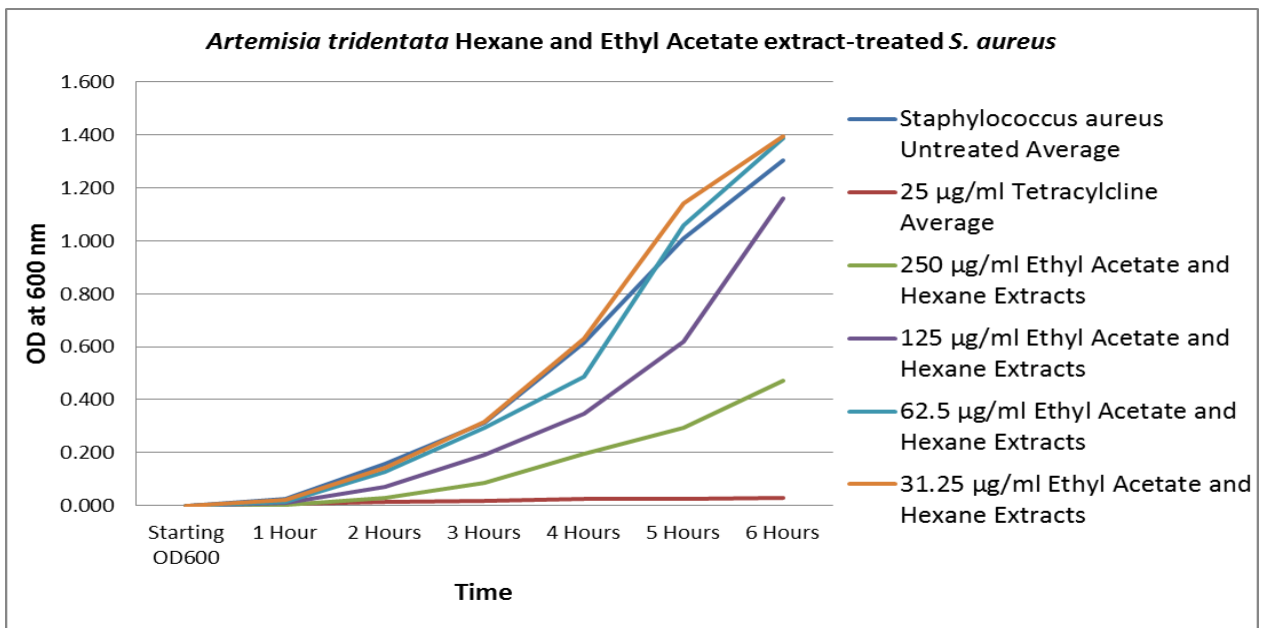

Figure 78: S. aureus treated with A. tridentata hexane and ethyl acetate extracts

The hexane and water extracts in combination also demonstrated significant growth inhibition at concentrations of 125 and $250 \mu \mathrm{g} / \mathrm{ml}$ each (figure 79). While the readings for this extract pairing at lesser concentrations were significantly larger than the average values from each extract alone, at $250 \mu \mathrm{g} / \mathrm{ml}$ each, diminishment was minimal. 


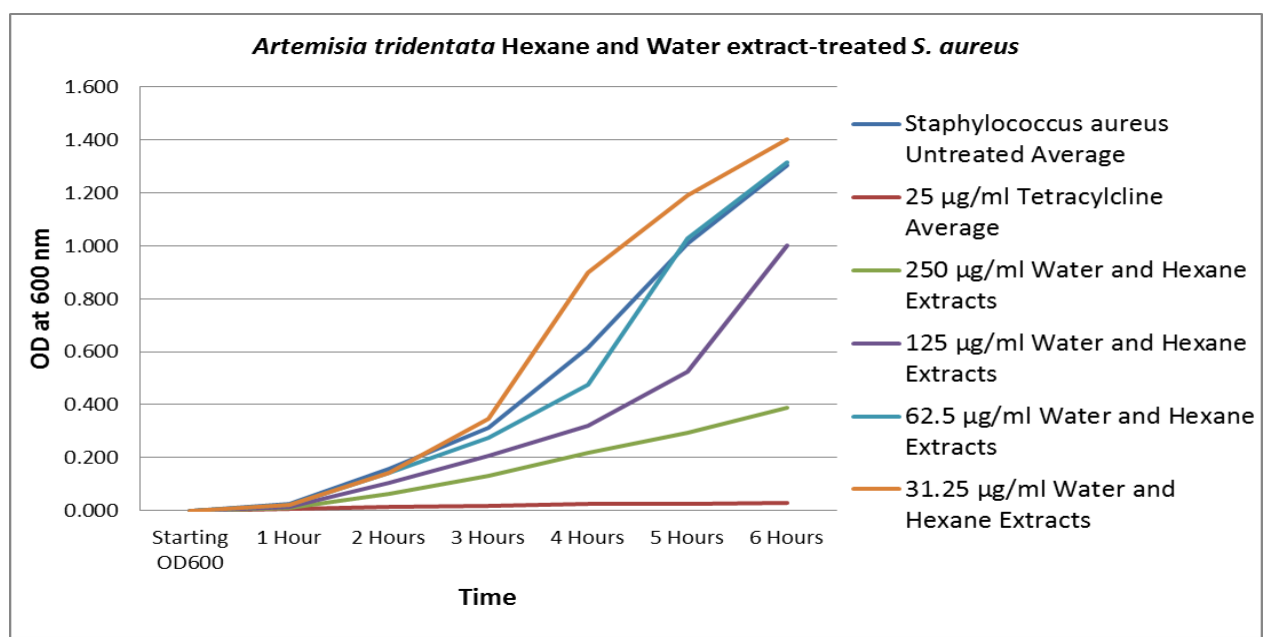

Figure 79: $S$. aureus treated with A. tridentata hexane and water extracts

Finally, in the case of the combination of the ethyl acetate and water extracts (figure 80), significant inhibition in comparison to the untreated control was observed at concentrations of $62.5 \mu \mathrm{g} / \mathrm{ml}$ and greater. Though there was a slight but statistically significant diminishment of antibacterial efficacy at extract concentrations of $250 \mu \mathrm{g} / \mathrm{ml}$ each in comparison to the averaged values of each extract at $500 \mu \mathrm{g} / \mathrm{ml}$, lower combinatorial values were similar to what was anticipated.

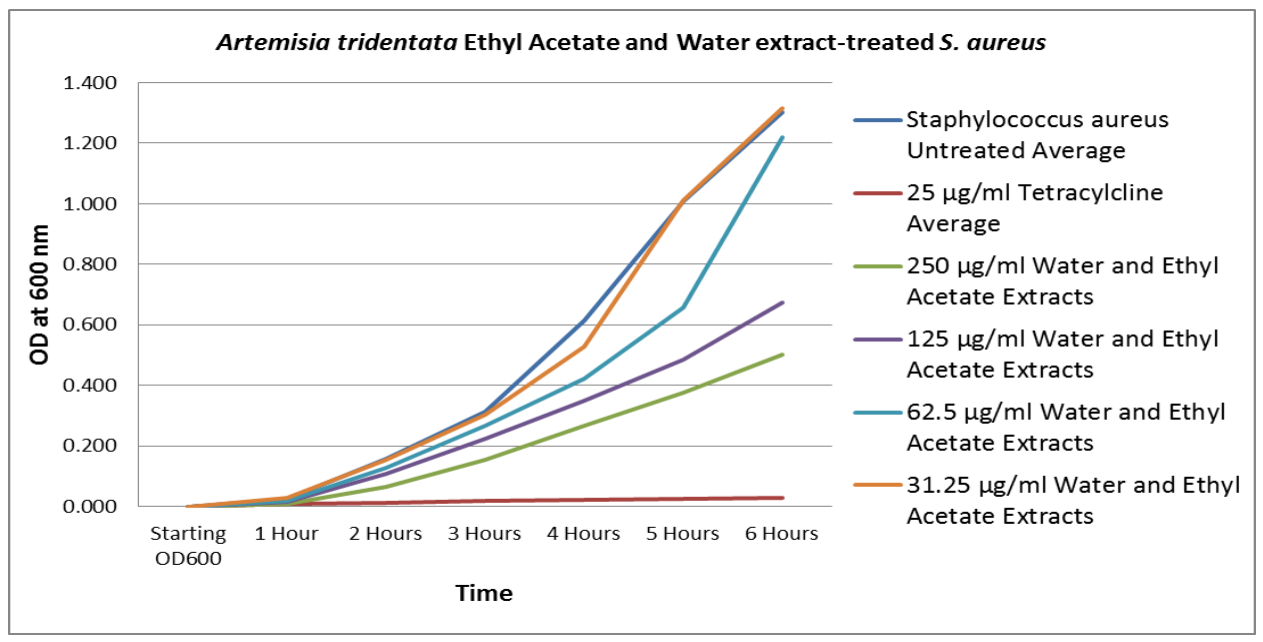

Figure 80: S. aureus treated with A. tridentata ethyl acetate and water extracts 


\section{Ampicillin-Resistant Staphylococcus aureus}

To determine if these extracts also possess growth inhibitory efficacy alone and/or in combination with a synthetic antibiotic versus a drug-resistant strain of Staphylococcus aureus, antibacterial assays were conducted using SA-BAA-44, an ampicillin-resistant strain of this bacterial species. Experimental conditions were similar to those described above for the previous extract/antibiotic combinatorial assays. However, extracts were only tested alone at concentrations of 500,250,125 and $62.5 \mu \mathrm{g} / \mathrm{ml}$, with the previously used lowest extract concentration of $31.25 \mu \mathrm{g} / \mathrm{ml}$ excluded. Also, ampicillin was tested alone at considerably higher concentrations of $800,400,200$ and $100 \mu \mathrm{g} / \mathrm{ml}$, as these levels were determined in a separate experiment as necessary to demonstrate intermediate growth inhibitory effects (data not shown). Each extract, at a concentration of $500 \mu \mathrm{g} / \mathrm{ml}$, was also tested in combination with each concentration of ampicillin. The method of statistical analysis employed, using the 6-hour time point readings, remained the same.

As can be seen in the following figures, growth of the untreated BAA-44 strain was substantially less than that of the antibiotic-susceptible strain, despite identical culture conditions. The hexane extract appears to have retained some inhibitory efficacy (figure 81), though this was only statistically significant at a concentration of $500 \mu \mathrm{g} / \mathrm{ml}$. The ethyl acetate extract (figure 82) was also only significantly effective at the maximum concentration assayed. The water extract (figure 83) demonstrated no significant antibacterial efficacy at any concentration. 


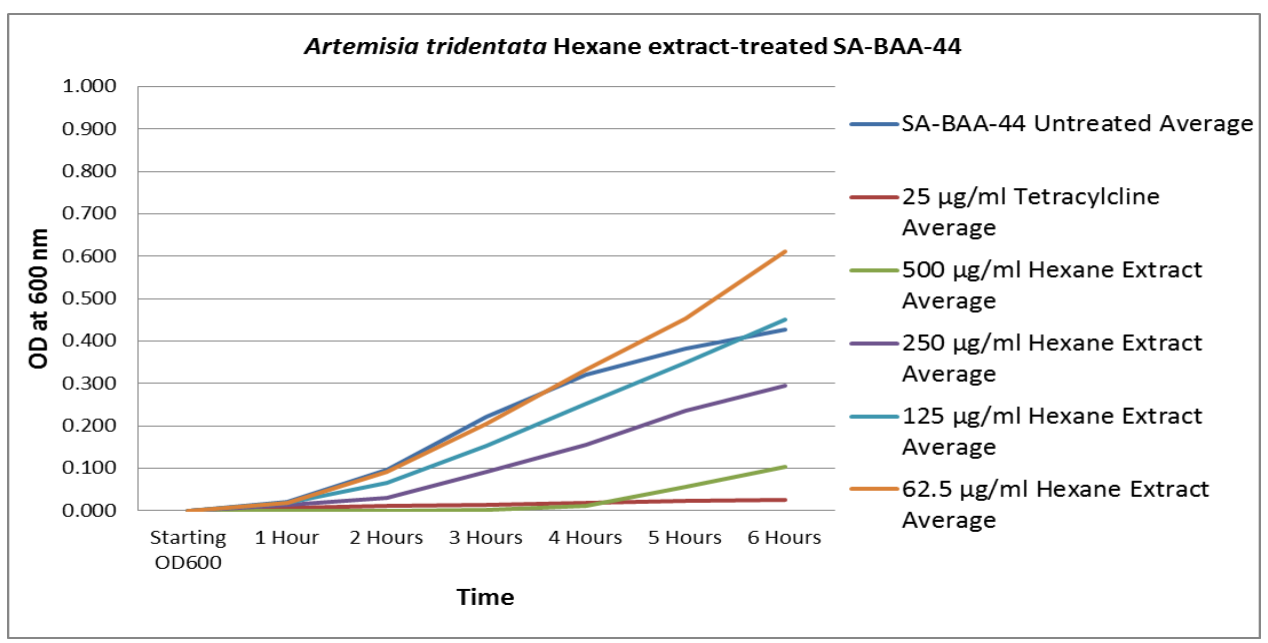

Figure 81: S. aureus BAA-44 treated with hexane extract

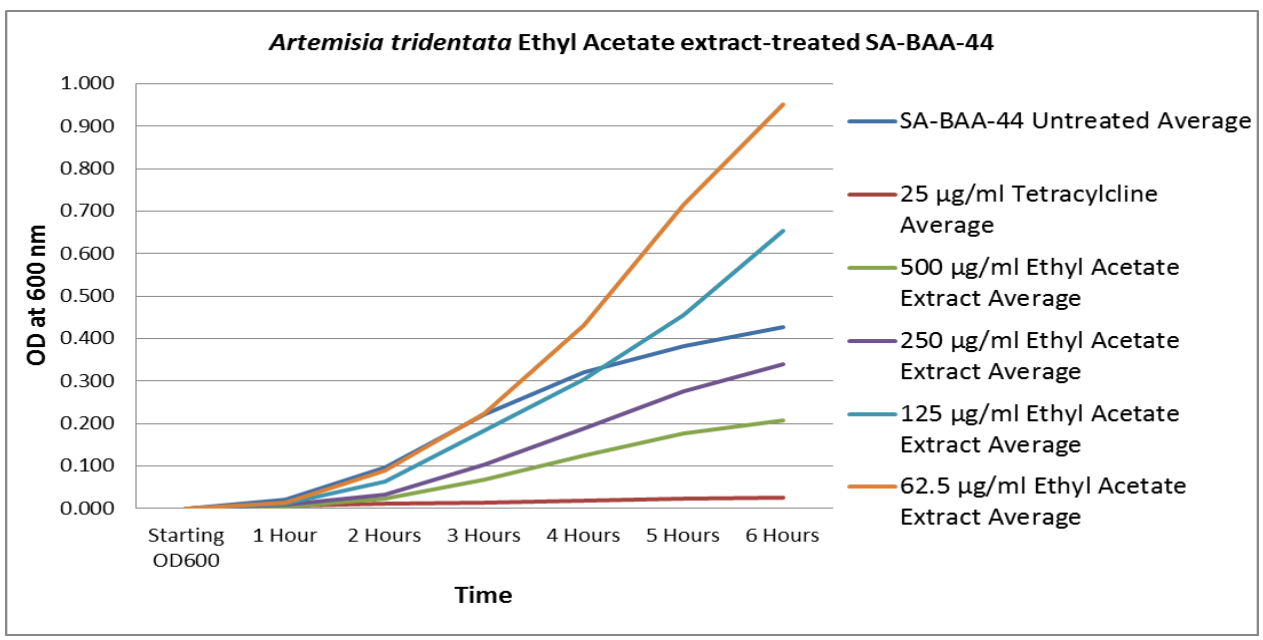

Figure 82: S. aureus BAA-44 treated with ethyl acetate extract 


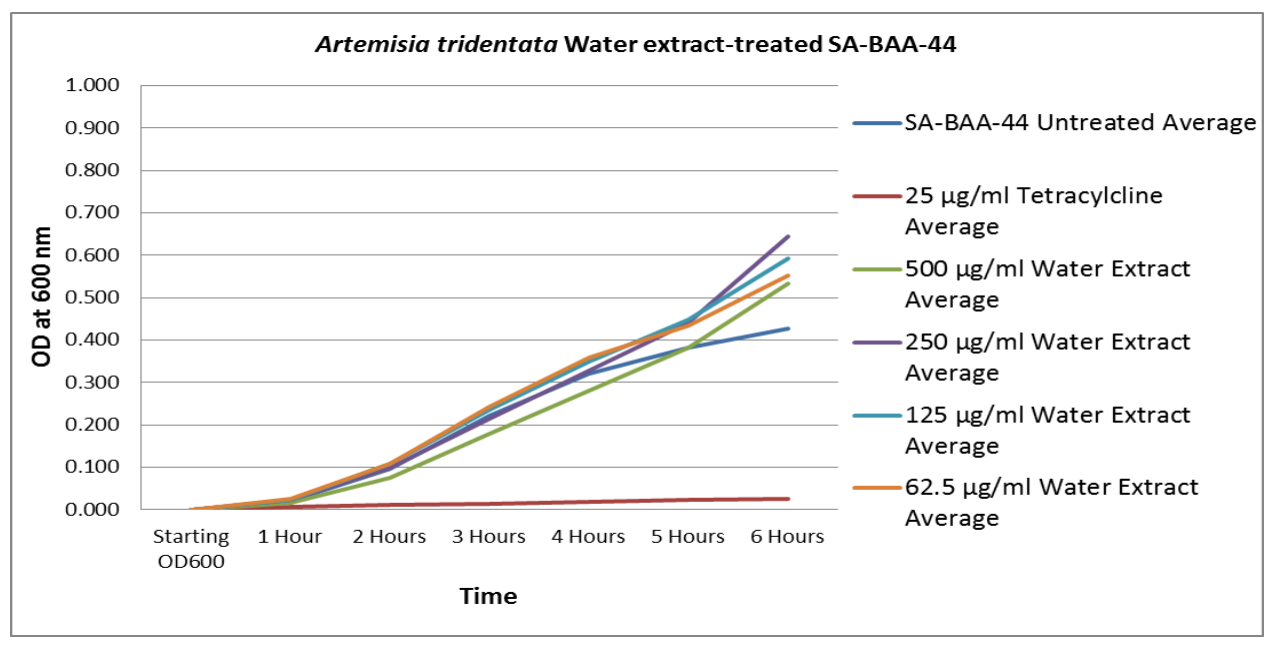

Figure 83: S. aureus BAA-44 treated with water extract

Ampicillin alone (figure 84) demonstrated statistically significant efficacy versus strain BAA-44 only at a concentration of $800 \mu \mathrm{g} / \mathrm{ml}$. However, in combination with the hexane extract at a concentration of $500 \mu \mathrm{g} / \mathrm{ml}$ (figure 85), growth inhibition became significant in comparison to both the untreated control and each ampicillin concentration alone, with growth curves resembling those of the positive control. In combination with the ethyl acetate extract (figure 86), growth inhibition again became statistically significant at all antibiotic concentrations using these comparisons, though the flattening of the growth curves of treated samples was less impressive than that of the hexane extract/antibiotic combinatorial samples. The water extract, by contrast, failed to demonstrate any statistically significant enhancement of antibiotic efficacy (figure 87). 


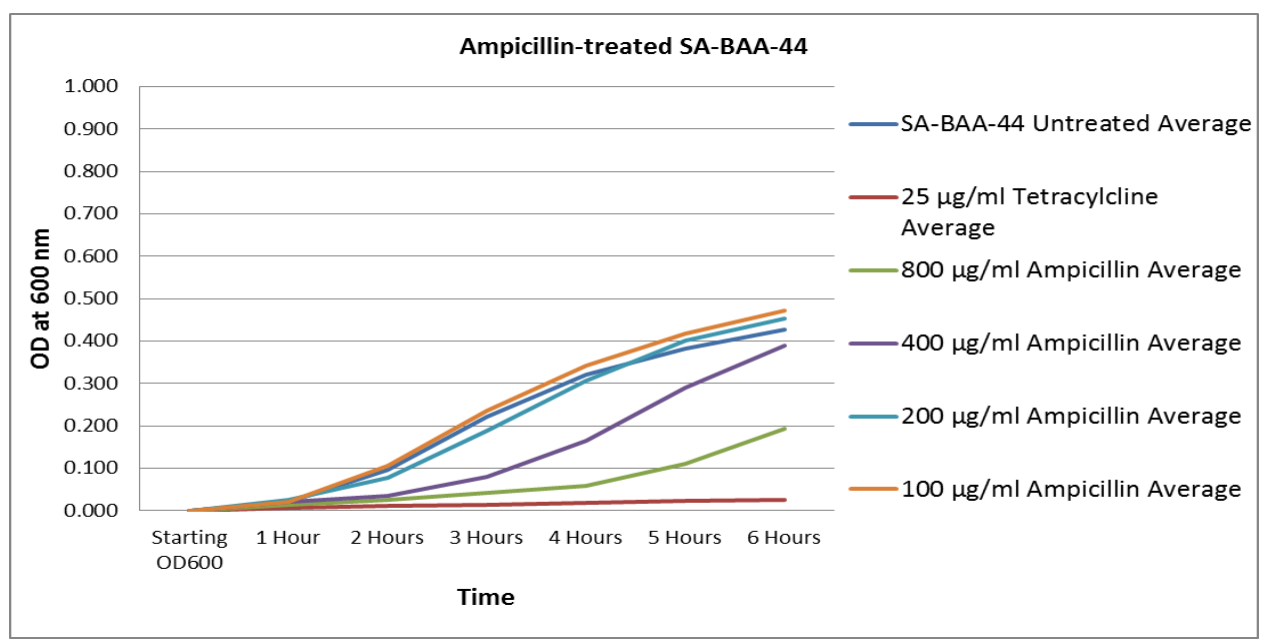

Figure 84: S. aureus BAA-44 treated with ampicillin

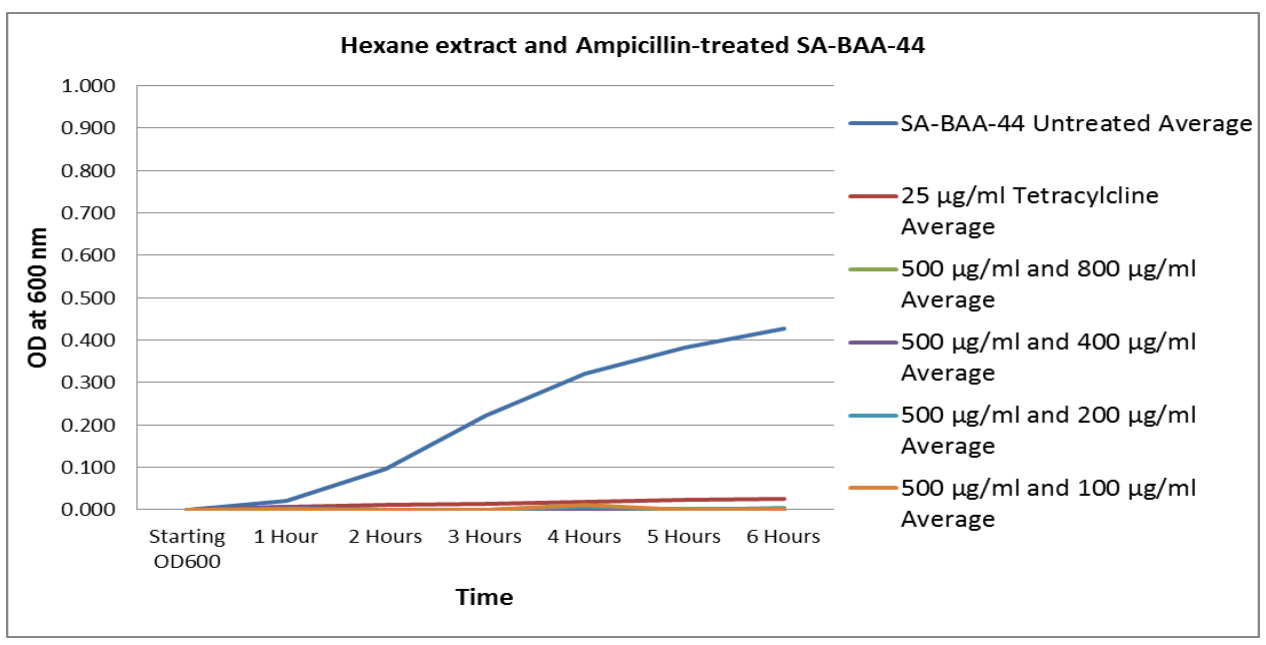

Figure 85: S. aureus BAA-44 treated with ampicillin and hexane extract 


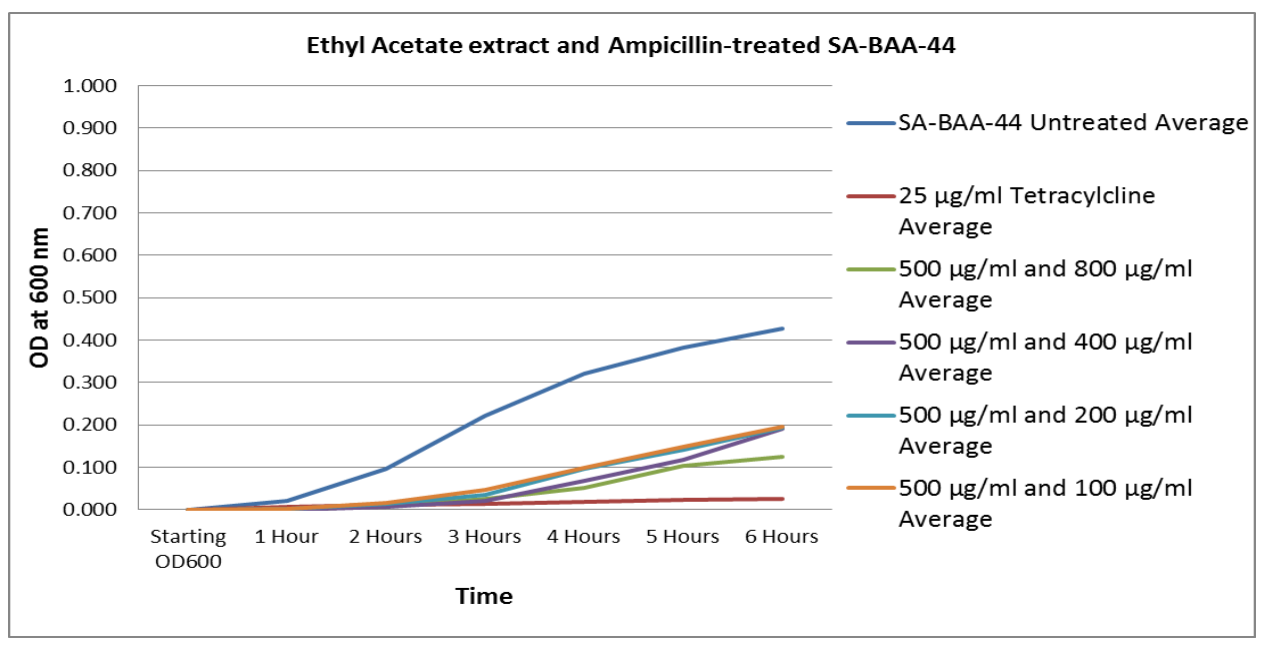

Figure 86: S. aureus BAA-44 treated with ampicillin and ethyl acetate extract

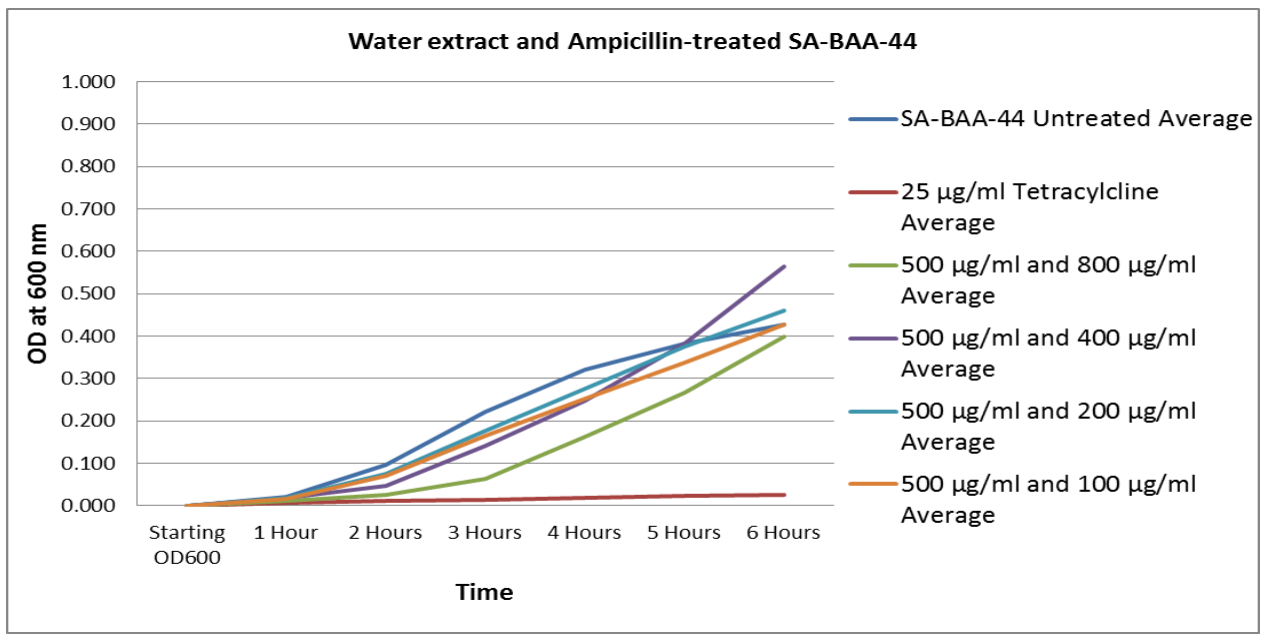

Figure 87: S. aureus BAA-44 treated with ampicillin and water extract 


\section{Biofilm Formation Assays}

In order to explore their potential in preventing Staphylococcus aureus biofilm formation on solid surfaces, the Artemisia tridentata extracts (hexane, ethyl acetate, water) were assayed for this ability using COSTAR brand 96-well polystyrene plates. All wells contained a final volume of $100 \mu \mathrm{l}$ prior to inoculation, and all sample types were prepared in triplicate. Two sets of wells were excluded from inoculation, and contained only LB. The first served to ensure sterility of the media and the plate, while the second served as a background control, measuring stain binding to the plate in the absence of a biofilm. Each extract was tested at concentrations of 500, 250 and $125 \mu \mathrm{g} / \mathrm{ml}$ in LB. DMSO in LB at $0.5 \%$ matched the dilution level of the highest extract concentration, and served as a vehicle control. Untreated samples served as a negative control, while $3 \%$ Nonidet P40 in LB served as a positive control. Overnight cultures of Staphylococcus aureus were diluted to $\mathrm{OD}_{600}$ levels of between 0.950 and 1.050 , as in previous assays. However, $5 \mu \mathrm{l}$ of this diluted culture was added to the appropriate wells on the plate, as 2 $\mu 1$ inoculums failed to demonstrate consistent biofilm formation in the untreated control samples in a previous assay (data not shown).

As the experimental design did not cause more than half the wells in the plate to be occupied, the setup was repeated a second time on the lower half of the plate, with the exception of the sterility control wells and the background stain binding control wells, thus providing two sets of samples in the event that one failed. The plate was incubated without rotation for approximately 10 hours at about $37^{\circ} \mathrm{C}$. Spectrophotometric readings of the plate were taken at $600 \mathrm{~nm}$ before and after incubation to detect media or plate 
contamination in the sterility control wells. Following incubation, the plate contents were decanted, and the plate rinsed three times in water. $100 \mu 1$ of a $0.1 \%$ crystal violet solution was added to the wells, and the plate incubated at room temperature for about 15 min. The plate contents were again decanted, the plate rinsed seven times in water, and the wells allowed to air-dry overnight in an open environment.

The following day, $100 \mu \mathrm{l}$ of a $95 \%$ ethanol solution was added to all of the wells except the sterility control wells, and a spectrophotometric measurement was taken at 595 $\mathrm{nm}$ to measure crystal violet retention. The averaged readings from the background stain binding control wells were subtracted from the values of the other samples, with negative numbers considered as readings of zero. The results from the six sets of samples are averaged in table 2 below, where it can be seen that none of the extracts demonstrated inhibition of Staphylococcus aureus biofilm formation under these experimental conditions. In fact, the extract-treated wells routinely retained more stain than did the untreated controls and DMSO-treated samples, with several results excluded due to readings too high to be accurately measured by the spectrophotometer. 


\begin{tabular}{|l|c|c|c|}
\cline { 2 - 4 } \multicolumn{1}{|c|}{} & $\begin{array}{c}\text { Starting } \\
\mathbf{O D}_{\mathbf{6 0 0}}\end{array}$ & $\mathbf{1 0 H r}$ & $\mathbf{O D}_{\mathbf{5 9 5}}$ \\
\hline Untreated Average & 0.100 & 1.326 & 1.232 \\
\hline Nonidet P40 3\% solution Average & 0.289 & 0.000 & 0.051 \\
\hline Staphylococcus aureus and 0.5\% DMSO Average & 0.106 & 1.358 & 1.363 \\
\hline Staphylococcus aureus and 500 ug/ml Hexane Extract Average & 0.300 & 1.082 & 1.853 \\
\hline Staphylococcus aureus and 250 ug/ml Hexane Extract Average & 0.197 & 1.247 & 1.953 \\
\hline Staphylococcus aureus and $125 \mathrm{ug} / \mathrm{ml}$ Hexane Extract Average & 0.151 & 1.392 & 1.681 \\
\hline Staphylococcus aureus and 500 ug/ml Ethyl Acetate Extract Average & 0.275 & 0.797 & 2.746 \\
\hline Staphylococcus aureus and 250 ug/ml Ethyl Acetate Extract Average & 0.188 & 1.173 & 2.759 \\
\hline Staphylococcus aureus and 125 ug/ml Ethyl Acetate Extract Average & 0.141 & 1.363 & 2.417 \\
\hline Staphylococcus aureus and 500 ug/ml Water Extract Average & 0.131 & 0.870 & 2.601 \\
\hline Staphylococcus aureus and 250 ug/ml Water Extract Average & 0.126 & 1.331 & 1.806 \\
\hline Staphylococcus aureus and 125 ug/ml Water Extract Average & 0.119 & 1.476 & 1.714 \\
\hline
\end{tabular}

Table 2: Biofilm formation assay results 


\section{Static Biofilm Assays}

Additional assays were performed to determine the antibacterial efficacy of the Artemisia tridentata extracts and the antibiotics ampicillin, amikacin or G418 sulfate, alone or in combination, versus static Staphylococcus aureus biofilms. As in the biofilm formation assays, COSTAR brand polystyrene 96-well polystyrene plates were used, and sample types were prepared in triplicate. $100 \mu \mathrm{LB}$ was added to all wells of the plate to be used in the assay. An overnight culture of Staphylococcus aureus was diluted in LB to an $\mathrm{OD}_{600}$ level between $0.950-1.050$, and $5 \mu 1$ of the diluted culture was added to all LBcontaining wells, excluding the first set of three. The plate was then incubated without rotation at approximately $37^{\circ} \mathrm{C}$ for about 10 hours. $\mathrm{OD}_{600}$ measurements of the first three wells, taken before and after incubation, served to ensure sterility of the media and the plate.

Following incubation, treatments were added to the static biofilms. The sterility control wells, to which no bacteria was added, also served to measure background stain binding to the plate in the absence of a biofilm. Therefore no treatment of these wells was necessary. The second and third sets of wells served as negative controls. To the second set of wells, nothing was added, while $100 \mu \mathrm{l}$ fresh LB was added to the wells of the third set.

As the extracts and antibiotics were to be added already pre-diluted to twice their desired final concentrations in $100 \mu$ l volumes of $\mathrm{LB}$, the purpose of this differential treatment of negative controls was to determine if leaving the untreated controls without an added $100 \mu \mathrm{l}$ fresh LB during the period of sample treatment would result in cell death. This could result in reduced biofilm stain binding, possibly masking extract or antibiotic 
biofilm-removal effects. Alternatively, adding $100 \mu \mathrm{l}$ LB could result in additional growth of the biofilm during the sample treatment period, possibly overstating extract or antibiotic effects. Therefore both possibilities needed to be explored. Each extract and antibiotic, pre-diluted in LB, was added and tested alone at final concentrations of 500 $\mu \mathrm{g} / \mathrm{ml}$ and $1 \mathrm{mg} / \mathrm{ml}$, respectively. Each extract and antibiotic were also tested in combination. Qiagen Lysis buffer, containing SDS and sodium hydroxide, served as a positive control. Following sample treatment, the plate was returned to incubation under the same conditions for approximately twelve hours.

The following day, the plate was washed and stained in a manner similar to the biofilm formation assays described above. As can be seen in table 3 below, results for the untreated controls were relatively similar regardless of the addition of fresh LB during sample treatment. However, none of the extracts or antibiotics by themselves, with the exception of ampicillin, demonstrated any reduction in stain retention in comparison to the untreated controls. Also, in combination, only the water extract applied together with ampicillin demonstrated any biofilm reduction, but this result was almost identical to that of ampicillin alone. Thus, the assay was abandoned after two repetitions. 


\begin{tabular}{|c|c|c|c|}
\hline & $\begin{array}{c}\text { Starting } \\
\mathrm{OD}_{600}\end{array}$ & $10 \mathrm{Hr}$ & $\mathrm{OD}_{595}$ \\
\hline Untreated Average (no LB added) & 0.111 & 1.180 & 0.374 \\
\hline Untreated Average (LB added) & 0.114 & 1.258 & 0.362 \\
\hline Staphylococcus aureus and Lysis Buffer Average & 0.126 & 1.319 & 0.000 \\
\hline Staphylococcus aureus and $500 \mathrm{ug} / \mathrm{ml}$ Hexane Extract Average & 0.112 & 1.153 & 0.386 \\
\hline Staphylococcus aureus and $500 \mathrm{ug} / \mathrm{ml}$ Ethyl Acetate Extract Average & 0.109 & 1.231 & 0.919 \\
\hline Staphylococcus aureus and 500 ug/ml Water Extract Average & 0.114 & 1.291 & 0.429 \\
\hline Staphylococcus aureus and 1 mg/ml Ampicillin Average & 0.110 & 1.209 & 0.329 \\
\hline Staphylococcus aureus and $1 \mathrm{mg} / \mathrm{ml}$ G418 Sulfate Average & 0.110 & 1.273 & 0.534 \\
\hline Staphylococcus aureus and $1 \mathrm{mg} / \mathrm{ml}$ Amikacin Average & 0.113 & 1.312 & 0.651 \\
\hline $500 \mu \mathrm{g} / \mathrm{ml}$ Hexane Extract and $1 \mathrm{mg} / \mathrm{ml}$ Ampicillin Average & 0.112 & 1.380 & 0.462 \\
\hline $500 \mu \mathrm{g} / \mathrm{ml}$ Hexane Extract and $1 \mathrm{mg} / \mathrm{ml}$ G418 Sulfate Average & 0.109 & 1.261 & 0.607 \\
\hline $500 \mu \mathrm{g} / \mathrm{ml}$ Hexane Extract and $1 \mathrm{mg} / \mathrm{ml}$ Amikacin Average & 0.106 & 1.259 & 0.712 \\
\hline $500 \mu \mathrm{g} / \mathrm{ml}$ Ethyl Acetate Extract and $1 \mathrm{mg} / \mathrm{ml}$ Ampicillin Average & 0.115 & 1.358 & 0.588 \\
\hline $500 \mu \mathrm{g} / \mathrm{ml}$ Ethyl Acetate Extract and $1 \mathrm{mg} / \mathrm{ml} \mathrm{G} 418$ Sulfate Average & 0.114 & 1.412 & 0.851 \\
\hline $500 \mu \mathrm{g} / \mathrm{ml}$ Ethyl Acetate Extract and $1 \mathrm{mg} / \mathrm{ml}$ Amikacin Average & 0.108 & 1.275 & 1.109 \\
\hline $500 \mu \mathrm{g} / \mathrm{ml}$ Water Extract and $1 \mathrm{mg} / \mathrm{ml}$ Ampicillin Average & 0.114 & 1.283 & 0.330 \\
\hline $500 \mu \mathrm{g} / \mathrm{ml}$ Water Extract and $1 \mathrm{mg} / \mathrm{ml}$ G418 Sulfate Average & 0.115 & 1.392 & 0.928 \\
\hline $500 \mu \mathrm{g} / \mathrm{ml}$ Water Extract and $1 \mathrm{mg} / \mathrm{ml}$ Amikacin Average & 0.111 & 1.489 & 1.191 \\
\hline
\end{tabular}

Table 3: Static biofilm assay results 
Serial Dilution and Plating

In an effort to determine whether the Artemisia tridentata extract antibacterial effects observed in earlier assays were bactericidal or bacteriostatic in nature, samples of Staphylococcus aureus cultures were serially diluted and plated after extract treatment to perform viable cell counts. Overnight cultures were first diluted to an $\mathrm{OD}_{600}$ level of approximately 1.000. A $50 \mu 1$ sample was removed and mixed with a $450 \mu 1$ volume of LB for a 1:10 dilution. $50 \mu \mathrm{l}$ of this mixture was removed and mixed in another $450 \mu \mathrm{l}$ volume of LB, and the process repeated until a final $1 \times 10^{-6}$ dilution was achieved. The entirety of the last dilution was plated on an LB agar plate, and served as a measure of the number of Staphylococcus aureus cells represented by an $\mathrm{OD}_{600}$ measurement approximating 1.000 .

To test extract antibacterial effects, $10 \mu \mathrm{l}$ of the dilution of the overnight culture $\left(\mathrm{OD}_{600}\right.$ level of approximately 1.000) was added to each of three tubes containing an Artemisia tridentata extract (hexane, ethyl acetate, water) at a concentration of $500 \mu \mathrm{g} / \mathrm{ml}$ in $500 \mu \mathrm{l}$ volumes of $\mathrm{LB}$, thus keeping consistent with the inoculum to media ratio used in the 96-well plate antibacterial assays ( $2 \mu 1$ culture to $100 \mu 1 \mathrm{LB})$. A fourth tube containing tetracycline at a concentration of $25 \mu \mathrm{g} / \mathrm{ml}$, also in a $500 \mu \mathrm{l}$ volume of LB, served as a positive control, while two untreated tubes containing only $500 \mu \mathrm{LB}$ each served as negative controls. $1 \mathrm{ml} \mathrm{LB}$ in a sixth tube was included as a sterility control. All tubes were incubated at approximately $37^{\circ} \mathrm{C}$ and $125 \mathrm{rpm}$.

After about $25 \mathrm{~min}$, the extract-treated samples, the tetracycline-treated sample and one of the untreated samples were removed from incubation. This time point was chosen based on a previous assay which indicated the doubling time of Staphylococcus 
aureus under these experimental conditions to be approximately 35 min (data not shown). The objective was to sample prior to the doubling time, so that diminished colony counts in the extract-treated samples in comparison to the untreated control could be reasonably attributed to either a bactericidal or bacteriostatic effect. For example, if the extracttreated samples showed reduced numbers of viable cells in comparison to the untreated controls, this would be indicative of a bactericidal effect, whereas if the extract effect was bacteriostatic in nature, the cell count would still hypothetically approximate or match that of the untreated control if the samples were taken before the doubling time.

However, if the samples were taken after the doubling time, diminished counts in extracttreated samples in comparison to the untreated controls could be attributed either to a bacteriostatic effect, or to the bactericidal killing of only a portion of the initial inoculum, with the remaining cells continuing to multiply.

$50 \mu 1$ from each of the samples removed from incubation at this time point was serially diluted in the same manner as described above, to a final dilution level of $1 \times 10^{-}$ ${ }^{4}$, with the last of each of these dilutions $(500 \mu \mathrm{l})$ plated on LB agar plates. After a total of 2 hours, the second untreated sample and the sterility control were removed from incubation, with the untreated sample similarly diluted to a final dilution of $1 \times 10^{-5}$. The last dilution $(500 \mu \mathrm{l})$, as well as the sterility control $(1 \mathrm{ml})$, were also plated on LB agar plates. All plates were incubated overnight at approximately $37^{\circ} \mathrm{C}$, and the colony forming units counted the next day. The assay was repeated three times.

The results are tabulated in table 4 below. The colony counts for the extracttreated samples appear to have approximated those of the untreated control after $25 \mathrm{~min}$ of incubation. 


\begin{tabular}{|c|c|c|c|c|}
\hline & 1st Assay & 2nd Assay & 3rd Assay & Average \\
\hline Overnight Culture & $2.72 \times 10^{8}$ & $9.92 \times 10^{7}$ & $6.29 \times 10^{7}$ & $1.45 \times 10^{8}$ \\
\hline Untreated (25 min) & $6.64 \times 10^{6}$ & $3.00 \times 10^{6}$ & $1.72 \times 10^{6}$ & $3.79 \times 10^{6}$ \\
\hline Tetracycline [25 $\mu \mathrm{g} / \mathrm{ml}]$ & $6.00 \times 10^{4}$ & $2.00 \times 10^{4}$ & $4.40 \times 10^{5}$ & $1.73 \times 10^{5}$ \\
\hline Hexane extract [500 $\mu \mathrm{g} / \mathrm{m}]$ & $6.18 \times 10^{6}$ & $2.16 \times 10^{6}$ & $1.60 \times 10^{6}$ & $3.31 \times 10^{6}$ \\
\hline Ethyl acetate extract [500 $\mu \mathrm{g} / \mathrm{ml}]$ & $6.62 \times 10^{6}$ & $3.44 \times 10^{6}$ & $2.16 \times 10^{6}$ & $4.07 \times 10^{6}$ \\
\hline 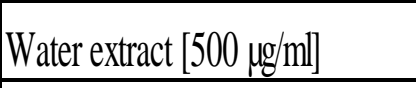 & $5.30 \times 10^{6}$ & $2.58 \times 10^{6}$ & $1.62 \times 10^{6}$ & $3.17 \times 10^{6}$ \\
\hline Untreated (2 hr) & $4.68 \times 10^{7}$ & $2.44 \times 10^{7}$ & $1.28 \times 10^{7}$ & $2.80 \times 10^{7}$ \\
\hline
\end{tabular}

Table 4: Serial and dilution of A. tridentata extract-treated S. aureus 


\section{Toxicity Assay}

To determine their potential toxicity to humans, a line of breast cancer cells (MDA-MB-231 ATCC-HTB-26) was treated with various concentrations of the Artemisia tridentata ethyl acetate extract. Cell samples $\left(5 \times 10^{5}\right)$ were cultured in Dulbecco's modified Eagle's medium (Fisher Scientific), containing 10\% fetal bovine serum, $0.1 \mathrm{mg} / \mathrm{mL}$ streptomycin, $100 \mathrm{U} / \mathrm{mL}$ penicillin, and $0.25 \mu \mathrm{g} / \mathrm{mL}$ amphotericin $\mathrm{B}$ at $37{ }^{\circ} \mathrm{C}$ in a humidified CO\#-controlled (5\%) incubator. The samples were then treated with various concentrations of the extract $(50,100,200,400$ or $600 \mathrm{ug} / \mathrm{ml})$ for a period of 24 hours. Untreated samples served as negative controls, while DMSO-treated samples $(0.1 \%)$ served as vehicle controls. Following extract treatment, cells were washed with PBS, and $2 \mathrm{ml}$ serum-free culture media containing $1 \mathrm{mg} / \mathrm{ml}$ of the tetrazolium salt [3(4,5-dimethylthiazol-2-yl)-2,5-diphenyltetrazolium bromide] (Sigma-Aldrich) was added to each well. After 4 hours, the media was discarded, and DMSO was added to dissolve MTT-derived formazan. The level of formazan was quantified by measurement of absorbance at a wavelength of $550 \mathrm{~nm}$. The assay was performed in triplicate.

The amount of formazan in extract-treated samples was calculated as a percentage of the formazan in the untreated controls. As can be seen in figure 88 , the percentage of viable cells, as indicated by conversion of the tetrazolium salt to formazan, decreased with increasing concentrations of the ethyl acetate extract, with concentrations of 400 and $600 \mu \mathrm{g} / \mathrm{ml}$ reducing the level of formazan converted to less than $10 \%$ of that of the untreated control. DMSO had little to no effect (data not shown). 


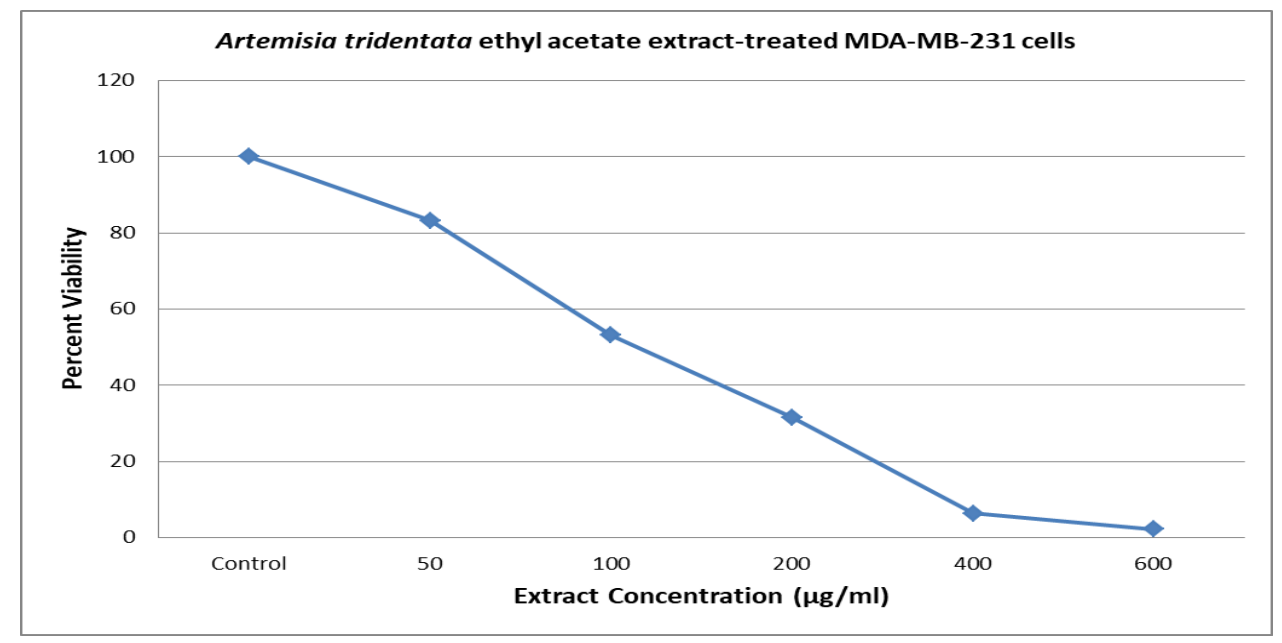

Figure 88: Artemisia tridentata ethyl acetate extract-treated MDA-MB-231 cells 


\section{pH Measurement}

The $\mathrm{pH}$ of extract-containing LB samples was measured to investigate the possibility that the antibacterial effects previously demonstrated were due to changes in media acidity or basicity. Each of the three Artemisia tridentata extracts (hexane, ethyl acetate, water) was diluted to a concentration of $500 \mu \mathrm{g} / \mathrm{ml}$ in a $50 \mathrm{ml}$ volume of $\mathrm{LB}$, and the $\mathrm{pH}$ of each sample measured with a Mettler Toledo "SevenEasy" meter. An equivalent volume of untreated LB from the same preparation was also tested to ensure the media used was in fact at or close to the expected value of $\mathrm{pH} 7$. The untreated LB provided a $\mathrm{pH}$ reading of 6.96 . The $\mathrm{pH}$ readings for the extract-containing samples were only slightly reduced, with measurements of $6.95,6.91$ and 6.90 for the hexane, ethyl acetate and water extracts, respectively. 


\section{Ethidium Bromide Assays}

Ethidium bromide binding assays, based on the work of MA Jabra-Rizk et al ${ }^{111}$, were performed to determine if the mechanism of action by which the extracts exert their antibacterial effect is through cell membrane disruption. Bacterial cell membrane disruption should result in the increased intercalation of ethidium bromide to bacterial DNA in cells exposed to this compound. As in the antibacterial assays, overnight cultures of Staphylococcus aureus were first diluted in $\mathrm{LB}$ to $\mathrm{OD}_{600}$ levels between 0.950-1.050. Several ml were centrifuged for $15 \mathrm{~min} @ 5 \mathrm{k} \mathrm{rpm}$, the supernatant removed, and the cells suspended in an equal volume of PBS.

$500 \mu 1$ of this cell suspension was added to $500 \mu \mathrm{l}$ PBS in each of six tubes. Three of these six tubes each contained one of the Artemisia tridentata extracts (hexane, ethyl acetate, water) at a concentration of $1 \mathrm{mg} / \mathrm{ml}$, yielding a final concentration of 500 $\mu \mathrm{g} / \mathrm{ml}$ following the addition of the cell suspension. The fourth tube contained DMSO at a concentration of $2 \%$, yielding a $1 \%$ final concentration following the addition of the cell suspension, and served as a vehicle control. A fifth tube containing only PBS served as a negative control, while the sixth contained the detergent Nonidet P40 at a concentration of $2 \%$, yielding a $1 \%$ final concentration following the addition of the cell suspension, and served as a positive control.

All samples were incubated at approximately $37^{\circ} \mathrm{C}$ and $125 \mathrm{rpm}$ for about $30 \mathrm{~min}$. $5 \mu \mathrm{g}$ ethidium bromide in an aqueous solution was then added to each, and the samples were allowed to incubate at room temperature for approximately $15 \mathrm{~min}$. Samples were then centrifuged for 15 min @ 5k rpm, after which the supernatants were removed, and the cell pellets washed in $1 \mathrm{ml}$ PBS each. The centrifugation step was repeated, the 
supernatants were again removed, and each pellet was suspended in $3 \mathrm{ml}$ PBS. Samples were then measured in a spectrofluorometer at $510 \mathrm{~nm}$ excitation and $605 \mathrm{~nm}$ emission to detect ethidium bromide binding to DNA. In an effort to account for possible differences in cell numbers, samples were then measured in a spectrophotometer at $600 \mathrm{~nm}$ to measure bacterial cell density. The assay was repeated in triplicate. As can be seen below in table 5, the spectrophotometer readings were used to adjust the emission measurements of each treated sample to that of the untreated control based on cell density, and a ratio of the emission of the sample to that of the untreated control was then calculated for each. Ethidium bromide binding to DNA was noticeably enhanced in the extract-treated samples, both in comparison to the negative control and the Nonidet P40treated positive control, with DMSO appearing to have little effect.

\begin{tabular}{|c|c|c|c|c|}
\cline { 2 - 5 } \multicolumn{1}{c|}{} & Emission & $\mathrm{OD}_{600}$ & $\begin{array}{c}\text { Adjusted } \\
\text { Value }\end{array}$ & Ratio \\
\hline Untreated & 23.490 & 0.062 & $\mathrm{NA}$ & $\mathrm{NA}$ \\
\hline $1 \%$ Nonidet P-40 & 106.783 & 0.121 & 55.520 & 2.286 \\
\hline DMSO & 14.697 & 0.049 & 20.205 & 0.888 \\
\hline Hexane extract & 211.633 & 0.137 & 93.610 & 3.950 \\
\hline Ethyl acetate extract & 228.412 & 0.126 & 112.913 & 4.670 \\
\hline Water extract & 49.013 & 0.116 & 25.412 & 1.148 \\
\hline
\end{tabular}

Table 5: Ethidium bromide binding assay results 


\section{DNA-nicking Assays}

In order to determine if the antibacterial effect of these extracts is the result of a mechanism of action involving DNA damage, a DNA-nicking assay was performed to observe for strand cleavage using a construct variant of plasmid pGEX. Plasmid DNA purified from bacteria is typically in its native supercoiled state. DNA strand damage results in a loss of supercoiling, which causes a slower migration of the plasmid when compared to the supercoiled form in an electrophoretic gel.

Five samples were prepared for each assay. To each sample, $2 \mu 1$ Fisher OPTIZYMETM 10X Buffer \#5 (10 mM Tris-HCl, pH 8.5, 10 mM MgCl $2,100 \mathrm{mM} \mathrm{KCl,}$ $0.1 \mathrm{mg} / \mathrm{ml} \mathrm{BSA}$ ) and $1 \mu \mathrm{l}$ plasmid were added. To three of the samples, an Artemisia tridentata extract (hexane, ethyl acetate, water) was added to a final concentration of 500 $\mu \mathrm{g} / \mathrm{ml}$. The fourth sample contained untreated plasmid and served as a negative control. To the fifth was added Fenton's reagent (10 $\mathrm{mM}$ ferrous ammonium sulfate, $10 \mathrm{mM}$ disodium EDTA, 9 mM hydrogen peroxide final concentrations) ${ }^{112}$, which served as a positive control. All samples contained a total of $20 \mu 1$, with DI water constituting the remaining volume in each.

Samples were incubated in a water bath at about $37^{\circ} \mathrm{C}$. After approximately 10 minutes, the positive control was removed and packed in ice. After a total incubation time of approximately 60 minutes, the remaining samples were removed, and $6 \mu 1$ of $4 \mathrm{X}$ loading dye was added to all samples. The entirety of each sample was loaded into the wells of a $1 \%$ agarose gel in $1 \mathrm{X}$ TAE buffer, and the gel run at $100 \mathrm{~V}$ for 90 minutes. The gel was stained with $30 \mu \mathrm{g}$ ethidium bromide in $50 \mathrm{ml}$ water for approximately 30 minutes, and photographed. The assay was repeated in triplicate. As can be seen in 
figures 89-91 below, treatment with the extracts did not appear to damage the DNA strands of the plasmid under the conditions assayed. From right to left, the lanes represent the DNA ladder ( $\lambda$-DNA-HindIII $/ \varphi \mathrm{X}-\mathrm{HaeIII})$, the untreated control, the Fenton's reagent-treated positive control, the hexane extract, ethyl acetate extract and water extract-treated samples. Band analysis performed using ImageJ software confirmed these negative results.

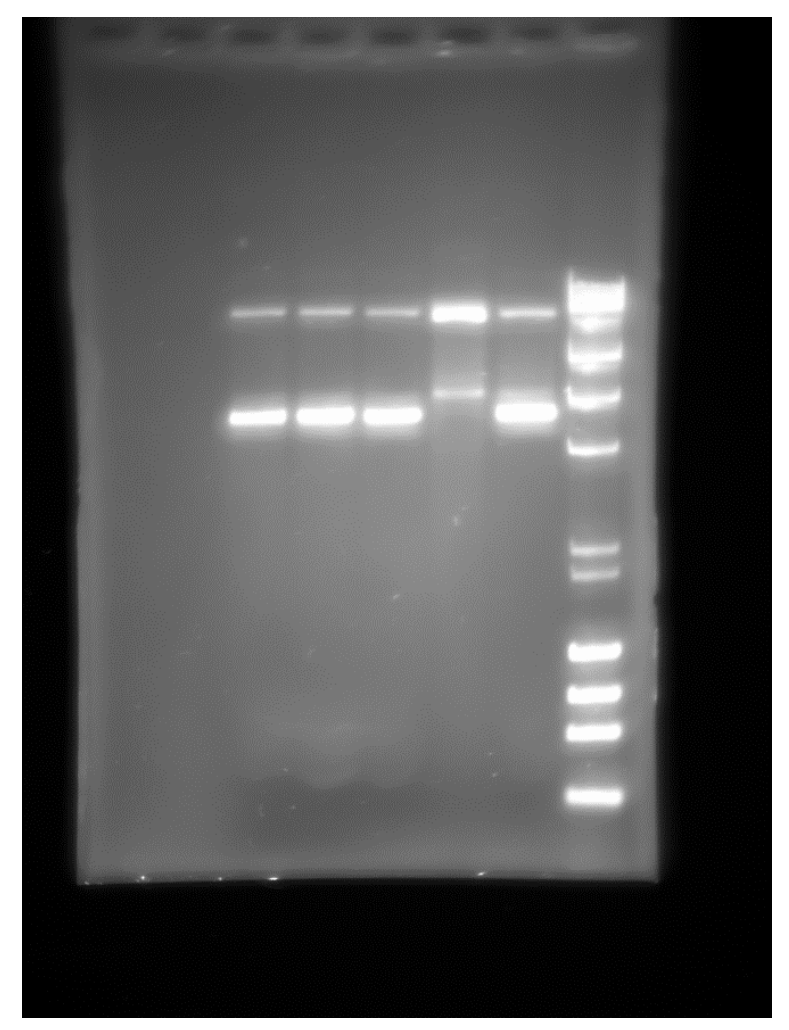

Figure 89: Gel electrophoresis of extract-treated pGEX ( $1^{\text {st }}$ assay $)$ 


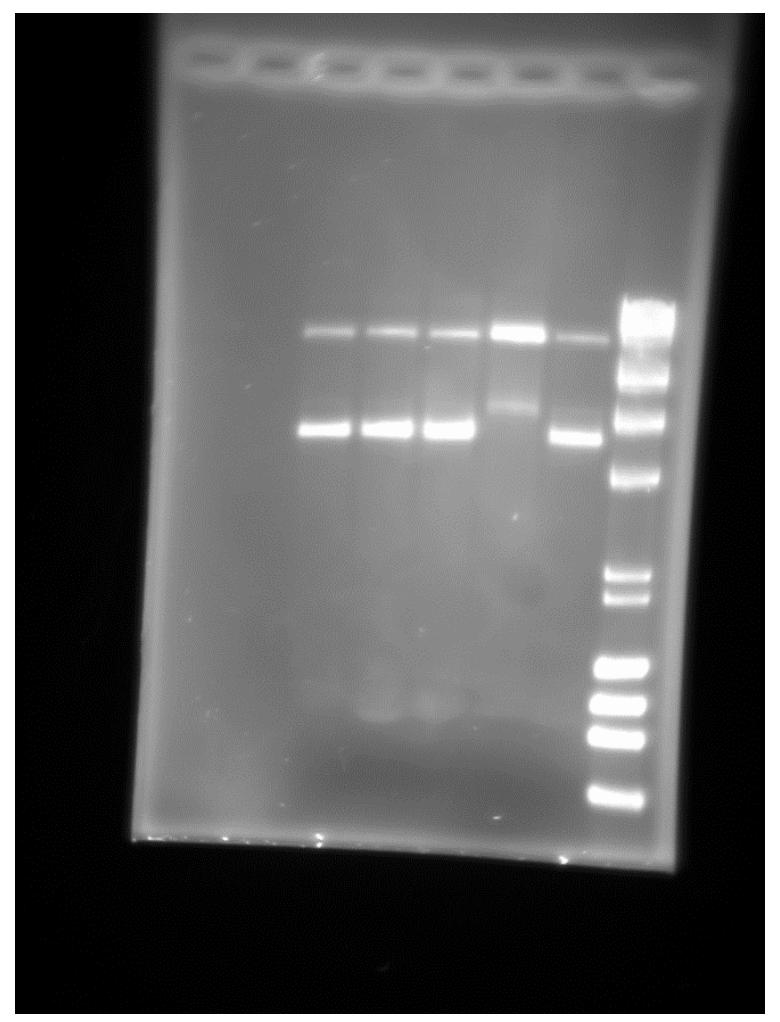

Figure 90: Gel electrophoresis of extract-treated pGEX (2nd assay) 


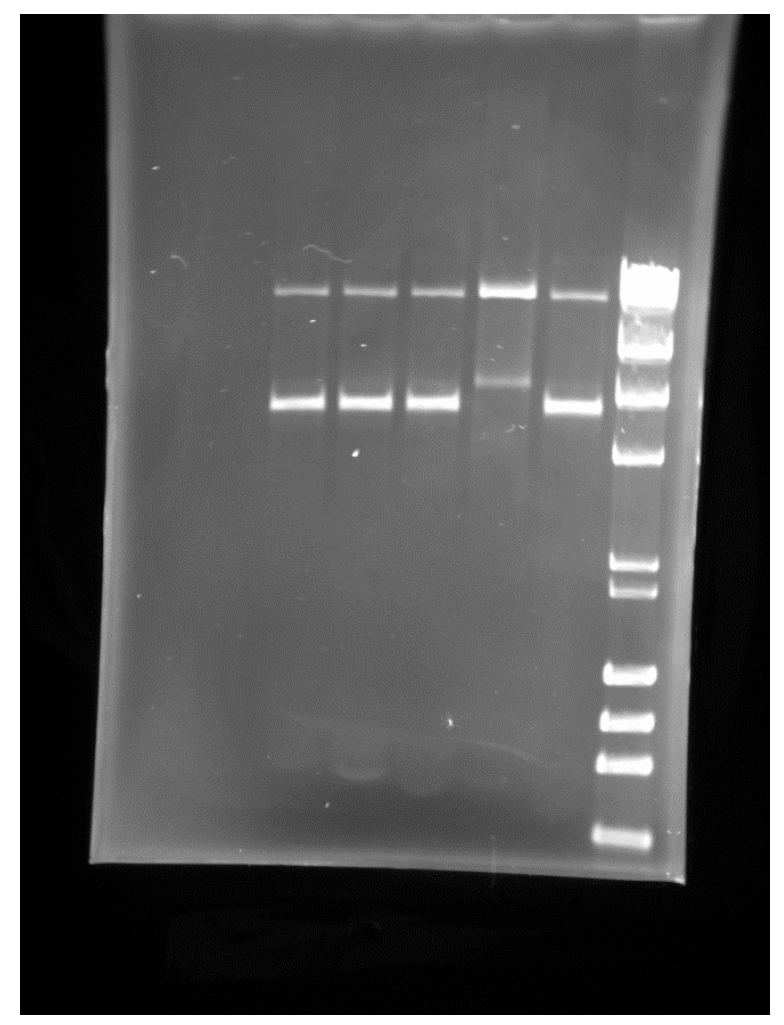

Figure 91: Gel electrophoresis of extract-treated pGEX (3rd assay) 


\section{Transmission Electron Microscopy}

Six $1 \mathrm{ml}$ samples of the ethyl acetate and water Artemisia tridentata extracts in LB were prepared, each at a final concentration of $500 \mu \mathrm{g} / \mathrm{ml}$. An overnight culture of Staphylococcus aureus was diluted to an $\mathrm{OD}_{600}$ reading approximating 1.000 , and $20 \mu \mathrm{l}$ of this was added to each extract-containing tube. The samples were then incubated for approximately 1 hour at about $37^{\circ} \mathrm{C}$ and $125 \mathrm{rpm}$. Following incubation, the tubes were centrifuged for $10 \mathrm{~min}$ at $8 \mathrm{k} \mathrm{rpm}$, the supernatants were removed, and the resultant pellets for each extract treatment were pooled and washed in $1 \mathrm{ml}$ PBS. The centrifugation was repeated a second time, and following removal of the supernatants, each pellet was fixed with $500 \mu 12.5 \%$ glutaraldehyde in PBS, and stored at $4^{\circ} \mathrm{C}$. Cells added to six tubes containing LB without extract underwent the same treatment, and served as a negative control.

A separate procedure was used for the hexane extract, as treated cells repeatedly failed to form an observable pellet using this protocol. In this case, $200 \mu 1$ cells were added to an extract-containing tube. The cells were washed five times with fresh LB, then fixed with $1 \mathrm{ml} 2.5 \%$ glutaraldehyde in phosphate buffer at $4^{\circ} \mathrm{C}$ for $1 \mathrm{~h}$. The cells were then washed three more times using phosphate buffer.

Prior to transmission electron microscopy (TEM), all samples were fixed with $1 \%$ osmium tetroxide for a period of 2 hours. This was followed by three 10 minute washings with phosphate buffer, and subsequent dehydration in a series of ethanol concentrations $(30 \%, 50 \%, 70 \%, 90 \%$ and $95 \%)$, for 15 min each. Osmified tissues were dehydrated in a 95\% ethanol/5\% acetone solution, followed by absolute acetone, and embedded in epoxy resin (Epon 812, Pelco-EEUU). Samples were then infiltrated and 
embedded in Spurr's resin (Epon 812, Pelco-EEUU). Ultrathin sections were cut with a diamond knife using an ultramicrotome (Ultracut equipment -Leitz), and then mounted on bare copper grids. Finally, specimens were counter stained with a 2\% (w/v) uranyl acetate and lead citrate solution for 3 minutes, then with a $0.25 \%(\mathrm{w} / \mathrm{v})$ solution for 2 minutes, and examined with a Zeiss EM 900 microscopy (Zeiss, Oberkochen, Germany). As can be seen in figure 92 below, transmission electron microscopy of the untreated control demonstrated intact cell walls and membranes. A similar result was observed for the water extract-treated cells (figure 93). However, after treatment with either the hexane (figure 94) or ethyl acetate (figure 95) extracts, Staphylococcus aureus cells showed an apparent absence of cytoplasmic contents and unique chromatin organization (i.e., non-condensed chromatin). Specifically, hexane extract-treated cells demonstrated damage to membrane integrity, resulting in a partial release of cellular content (indicated by arrowheads), while ethyl acetate extract-treated cells appear to have suffered cell wall disruption (indicated by asterisks). The residual cell walls appeared as bacterial "ghosts", with a complete absence of cytoplasmic contents, indicating cellular rupture. Interestingly, these ethyl acetate extract-treated cells also demonstrated cellular aggregation, or possible fusing of the cells. 


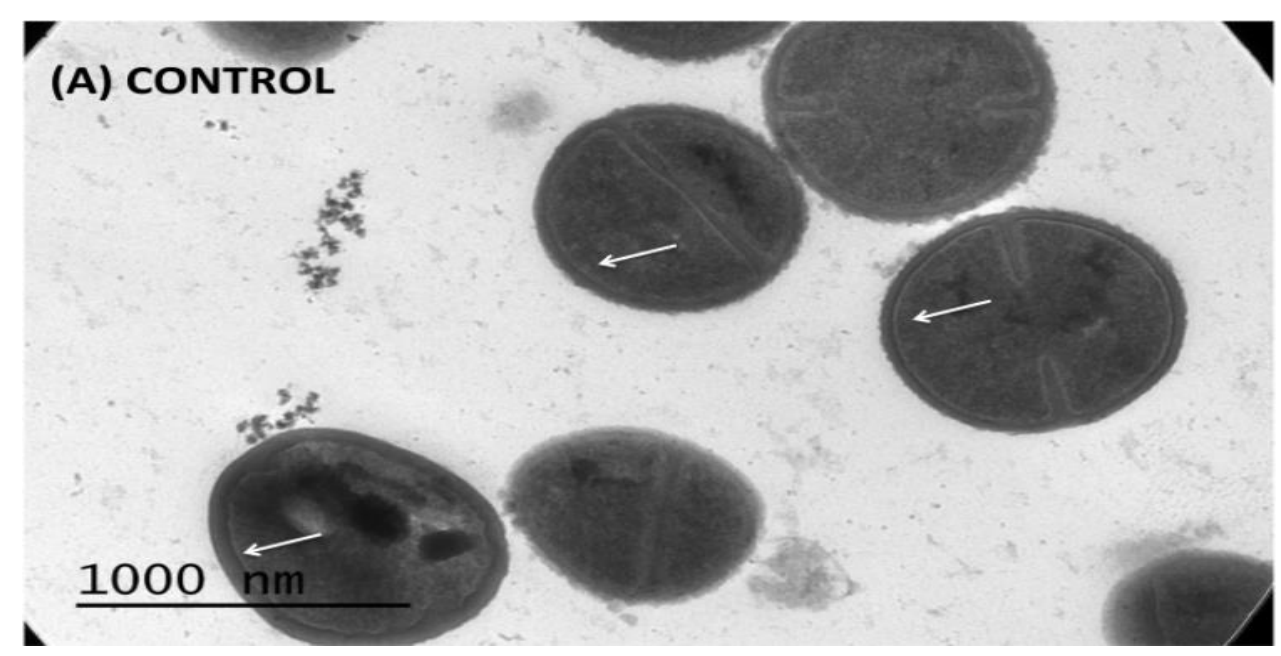

Figure 92: TEM image of untreated Staphylococcus aureus

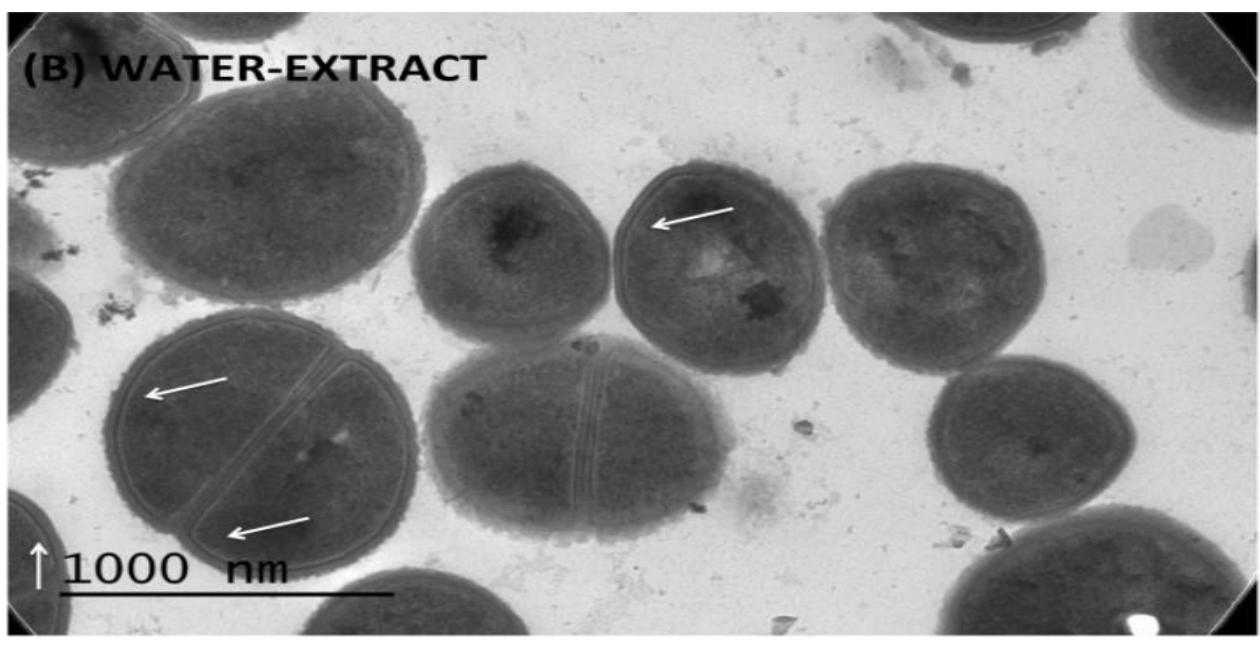

Figure 93: TEM image of water extract-treated Staphylococcus aureus 


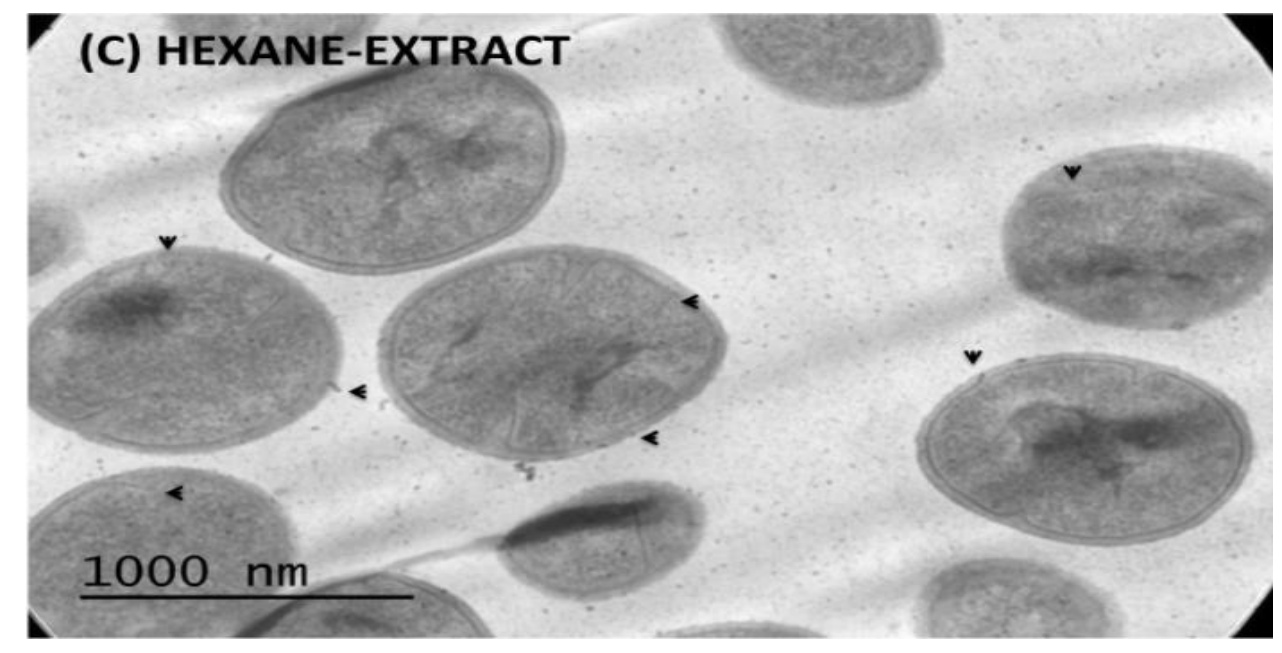

Figure 94: TEM image of hexane extract-treated Staphylococcus aureus

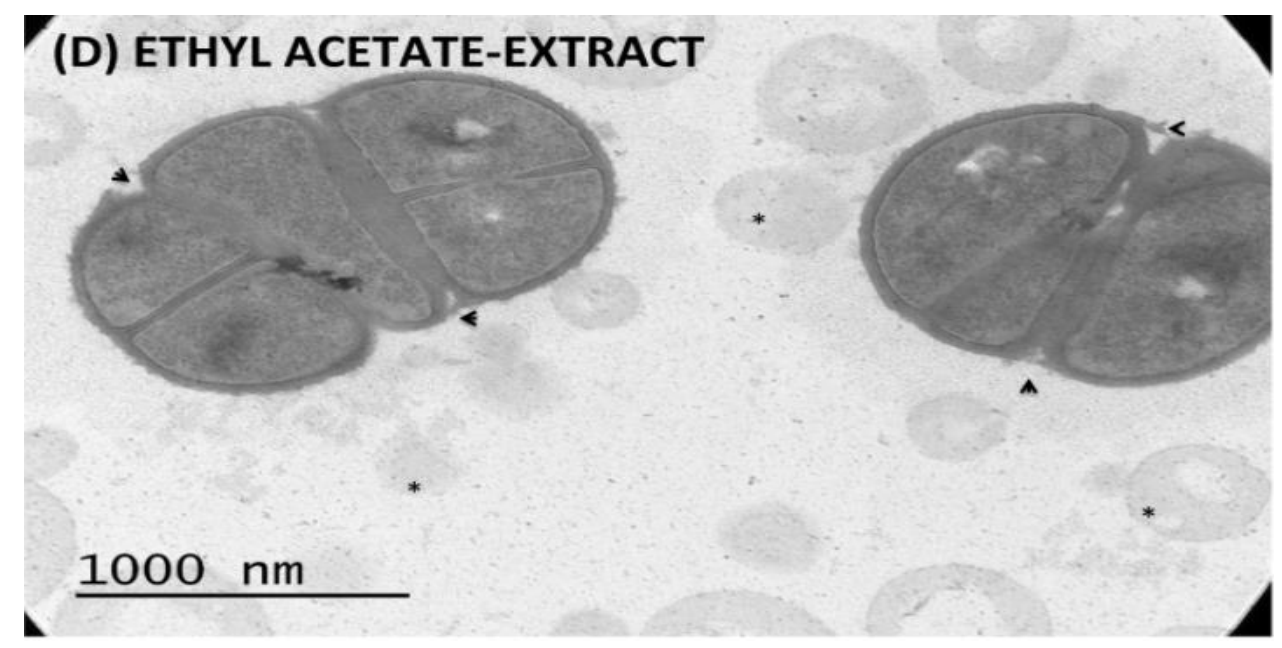

Figure 95: TEM image of ethyl acetate extract-treated Staphylococcus aureus 


\section{GC-MS Analysis}

Each of the three extracts was submitted for gas chromatography-mass spectrometry analysis to determine chemical composition. DMSO served as a control. $20 \mu 1$ of each sample was mixed with $50 \mu 1$ methanol and $50 \mu 1$ dichloromethane. Sample injection and gas chromatography were performed using an Agilent 6890 gas chromatograph equipped with an autosampler and an HP-5MS UI capillary column (30m, $0.250 \mathrm{~mm}, 0.25 \mu \mathrm{m}) .5 \mu \mathrm{l}$ of each sample was injected at an injection temperature of $320^{\circ} \mathrm{C}$, using an injection mode with a split 1:10 ratio. Helium served as the carrier gas at a constant rate flow of $0.9 \mathrm{ml} / \mathrm{min}$. The oven temperature was held at $60^{\circ} \mathrm{C}$ for $1 \mathrm{~min}$, then increased to $320^{\circ} \mathrm{C}$ at a rate of $5^{\circ} \mathrm{C}$, and held at this final temperature for $4 \mathrm{~min}$. An Agilent 5973 single-quadrupole mass spectrometer was used for detection using electron impact ionization operating in positive mode, operating under full scan mode (50-450 Da, $1 \mathrm{~Hz}$ ) with a solvent delay of $7 \mathrm{~min}$.

A total of 60 peaks were identified in the hexane extract sample (figure 96), 93 in the ethyl acetate extract sample (figure 97), and 87 in the water extract sample (figure 98). Spectral peaks were identified based using the NIST 1998 data library. 


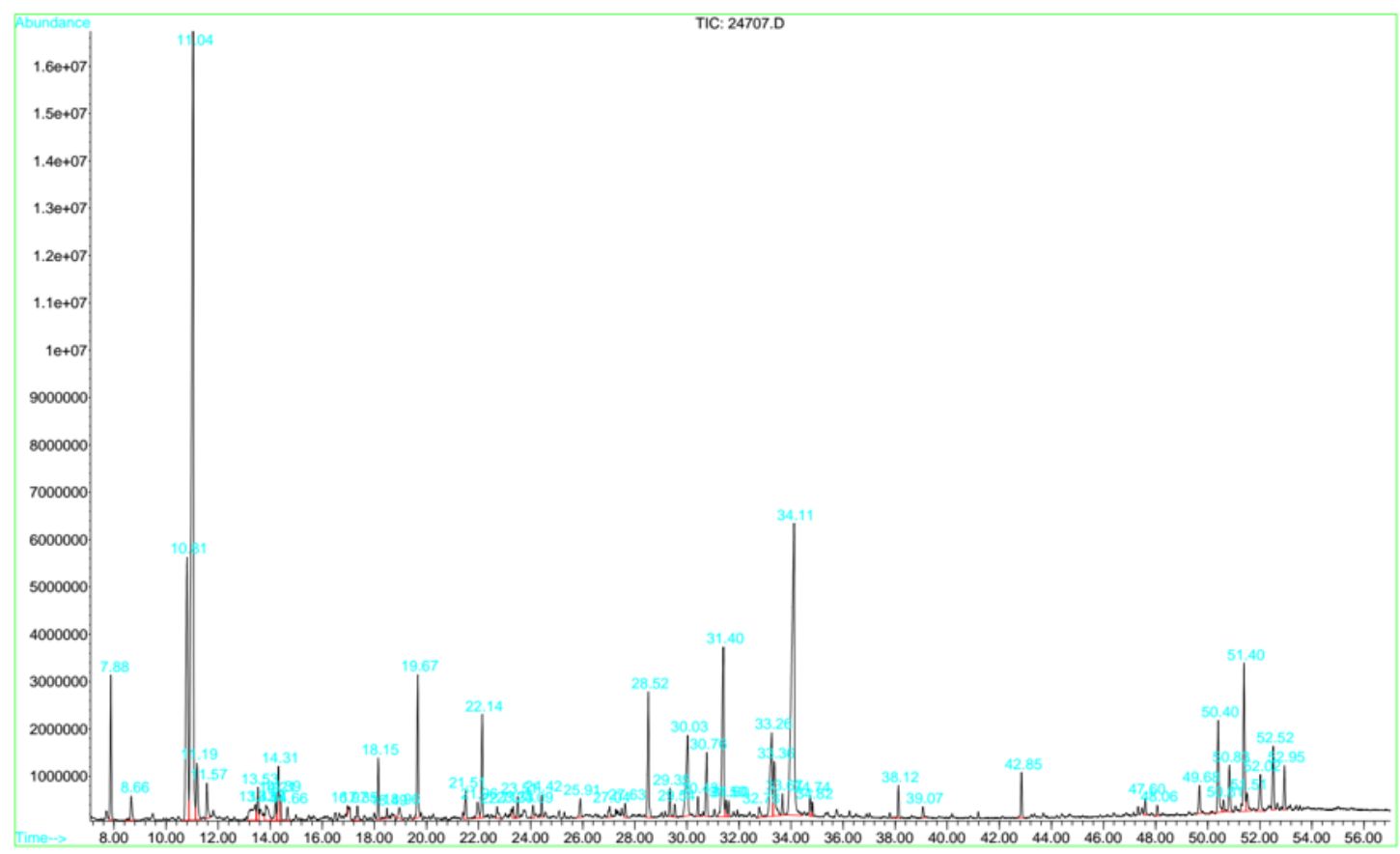

Figure 96: GC-MS spectral results for Artemisia tridentata hexane extract

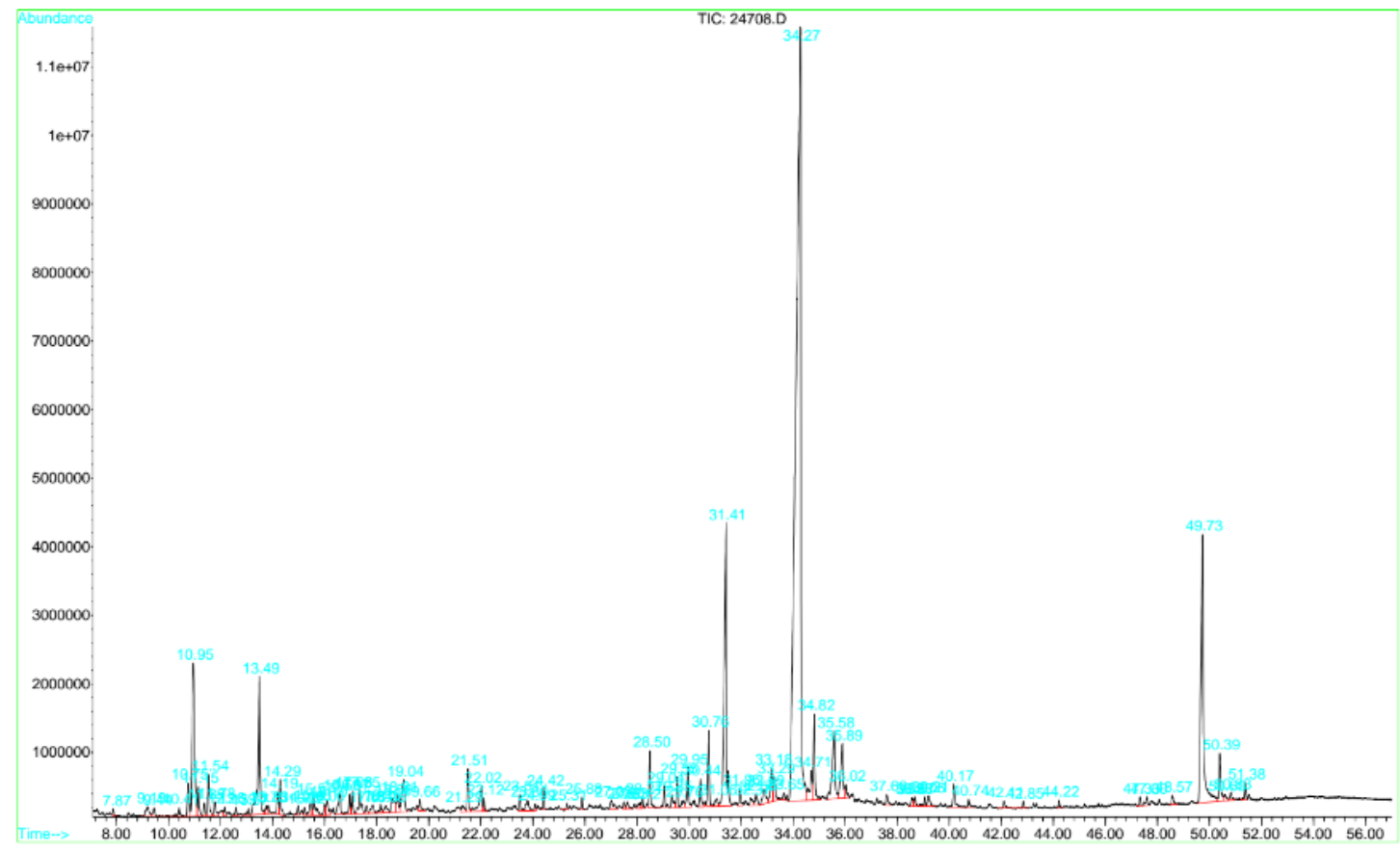

Figure 97: GC-MS spectral results for Artemisia tridentata ethyl acetate extract 


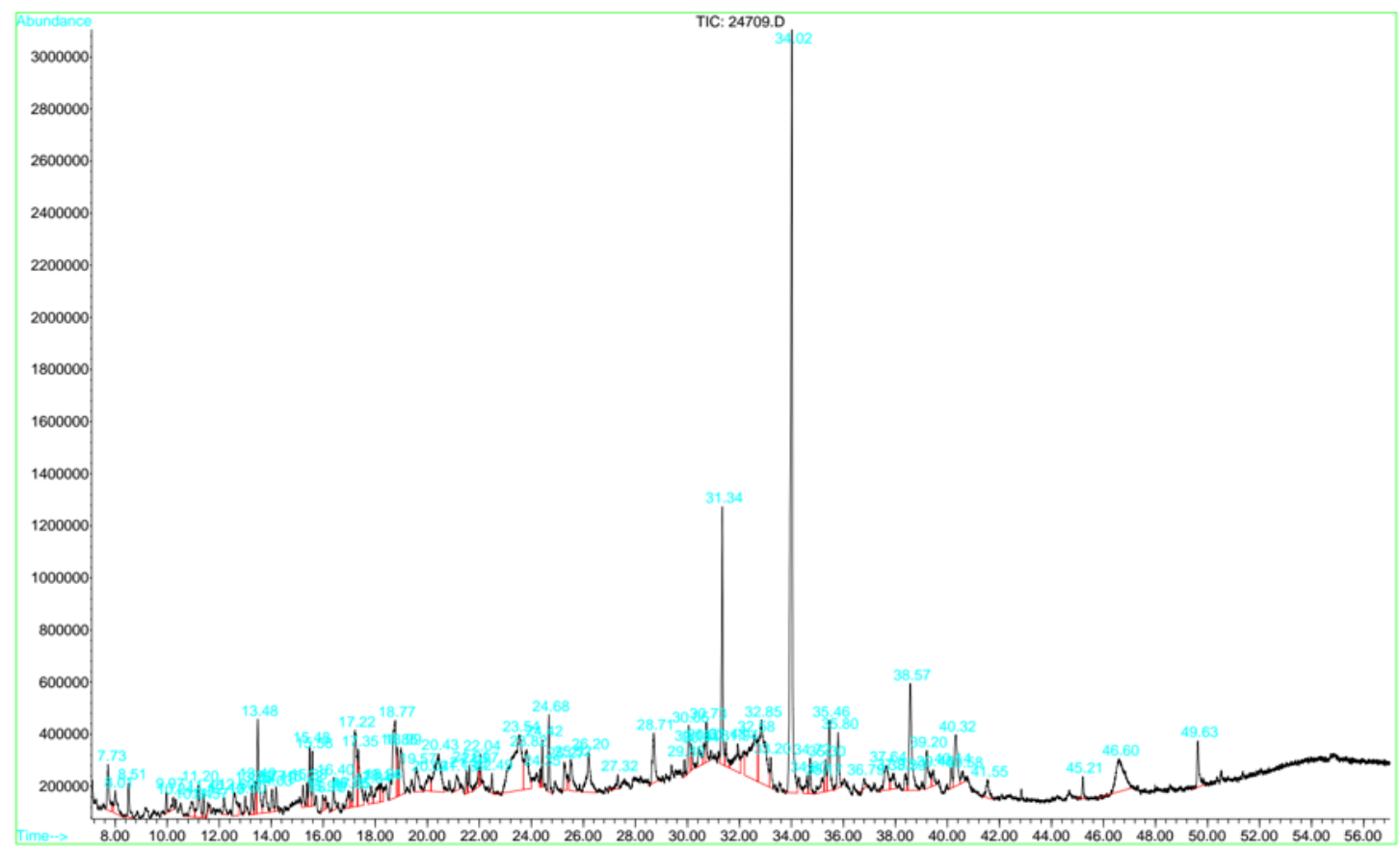

Figure 98: GC-MS spectral results for Artemisia tridentata water extract

Among the three extracts, compounds identified with a probability value of at least $50 \%$ and a peak area of at least $1 \%$ were tabulated and compared. 43 compounds in the hexane extract analysis met these criteria (table 6). Of these, seven had peak areas of at least $5 \%$. Three of these seven are tentative identifications of the peak detected at the retention time of 11.04 minutes, with a peak area of $24.93 \%$ and probable identification values of at least $90 \%$. These three are stereoisomer variations of the first listed compound, a terpene commonly known as "camphor." The next two compounds, with a retention time of 34.12 minutes, a peak area of $13.35 \%$, and also with probable identification values of at least $90 \%$, are again stereoisomers of the same compound, a sesquiterpene lactone with the molecular formula $\mathrm{C}_{15} \mathrm{H}_{18} \mathrm{O}_{3}$, commonly referred to as "achillin" or "azuleno." The final two compounds are again possible identifications of the same peak, with a retention time 10.81 minutes and a peak area of $8.49 \%$, tentatively 
labeled as either "exo-2-bromonoborane," or "Bicyclo[2.2.1]heptane, 2-(2-propenyl)-," the latter of which is also known as "2-(2-Methyl-1-propenyl)-bicyclo[2.2.1]heptane." However, these two compounds have probable identification values of only 50 and $59 \%$, respectively. 


\begin{tabular}{|c|c|c|c|c|c|}
\hline \multicolumn{6}{|c|}{ Hexane Extract GC-MS Results } \\
\hline $\begin{array}{c}\text { Peak } \\
\text { Number }\end{array}$ & $\begin{array}{l}\text { Retention } \\
\text { Time }\end{array}$ & Area & Library/ID & CAS\# & $\begin{array}{c}\text { Probable } \\
\text { Identification }\end{array}$ \\
\hline 4 & 11.04 & 24.93 & Camphor & $000076-22-2$ & 90 \\
\hline 4 & 11.04 & 24.93 & Bicyclo[2.2.1] heptan-2-one, 1,7,7- & $000464-48-2$ & 97 \\
\hline 4 & 11.04 & 24.93 & Bicyclo[2.2.1] heptan-2-one, 1,7,7- & $000464-49-3$ & 96 \\
\hline 44 & 34.12 & 13.35 & Azuleno[4,5-b]furan-2,7-dione, 3,3 & 005956-04-7 & 91 \\
\hline 44 & 34.12 & 13.35 & Achillin & $071616-00-7$ & 90 \\
\hline 3 & 10.81 & 8.49 & exo-2-Bromonorbornane & $002534-77-2$ & 50 \\
\hline 3 & 10.81 & 8.49 & Bicyclo[2.2.1]heptane, 2-(2-propen & 002633-80-9 & 59 \\
\hline 37 & 31.40 & 4.68 & Unidentified & NA & NA \\
\hline 56 & 51.40 & 3.30 & .alpha.-Amyrin & $000638-95-9$ & 91 \\
\hline 56 & 51.40 & 3.30 & .beta.-Amyrin & $000559-70-6$ & 90 \\
\hline 56 & 51.40 & 3.30 & beta.-Amyrin trimethylsilyl ether & 001721-67-1 & 56 \\
\hline 41 & 33.26 & 2.79 & 9,12-Octadecadienoic acid (Z,Z)- & $000060-33-3$ & 99 \\
\hline 41 & 33.26 & 2.79 & 9,12-Octadecadienoic acid, methyl & $002462-85-3$ & 76 \\
\hline 41 & 33.26 & 2.79 & 2-Chloroethyl linoleate & $025525-76-2$ & 87 \\
\hline 34 & 30.03 & 2.74 & Pentadecanoic acid & $001002-84-2$ & 76 \\
\hline 34 & 30.03 & 2.74 & n-Hexadecanoic acid & $000057-10-3$ & 89 \\
\hline 34 & 30.03 & 2.74 & Tridecanoic acid & 000638-53-9 & 78 \\
\hline 19 & 19.67 & 2.70 & Benzene, 1-(1,5-dimethyl-4-hexenyl & 000644-30-4 & 99 \\
\hline 19 & 19.67 & 2.70 & 6-(p-Tolyl)-2-methyl-2-heptenol & 039599-18-3 & 59 \\
\hline 31 & 28.52 & 2.53 & 3,5-Di-tert-butylbenzoic acid & $016225-26-6$ & 59 \\
\hline 1 & 7.88 & 2.33 & Eucalyptol & $000470-82-6$ & 95 \\
\hline 1 & 7.88 & 2.33 & Trifluoroacetyl-.alpha.-terpineol & $1000058-17-6$ & 62 \\
\hline 53 & 50.40 & 2.06 & .beta.-Sitosterol & $000083-46-5$ & 50 \\
\hline 53 & 50.40 & 2.06 & gamma.-Sitosterol & $000083-47-6$ & 99 \\
\hline 53 & 50.40 & 2.06 & Stigmasterol, 22,23-dihydro- & $1000214-20-7$ & 93 \\
\hline 22 & 22.14 & 1.85 & Caryophyllene oxide & $001139-30-6$ & 81 \\
\hline 22 & 22.14 & 1.85 & Caryophyllene oxide & $1000156-32-9$ & 94 \\
\hline 42 & 33.36 & 1.81 & 9,12,15-Octadecatrien-1-ol, (Z,Z,Z & $000506-44-5$ & 83 \\
\hline 42 & 33.36 & 1.81 & $9,12,15$-Octadecatrienoic acid, met & $000301-00-8$ & 74 \\
\hline 42 & 33.36 & 1.81 & Bicyclo[10.1.0]tridec-1-ene & 054766-91-5 & 53 \\
\hline 5 & 11.19 & 1.52 & Unidentified & NA & NA \\
\hline 59 & 52.52 & 1.40 & beta.-Amyrin & $000559-70-6$ & 53 \\
\hline 59 & 52.52 & 1.40 & .alpha.-Amyrin & 000638-95-9 & 83 \\
\hline 36 & 30.76 & 1.39 & Naphtho[1,2-b]furan-2,8(3H,4H)-dio & 013902-54-0 & 59 \\
\hline 7 & 13.43 & 1.20 & exo-2-Bromonorbornane & $002534-77-2$ & 59 \\
\hline 7 & 13.43 & 1.20 & Cyclohexene, 3-methyl-6-(1-methyle & $005256-65-5$ & 64 \\
\hline 7 & 13.43 & 1.20 & 2-Butanone, 4-cyclopentylidene- & 051004-21-8 & 64 \\
\hline 11 & 14.32 & 1.19 & Cyclobutane, 1,2-diethenyl-3,4-dim & $1000034-00-1$ & 59 \\
\hline 16 & 18.15 & 1.04 & Caryophyllene & $000087-44-5$ & 91 \\
\hline 16 & 18.15 & 1.04 & Bicyclo[7.2.0]undec-4-ene, $4,11,11$ & $000118-65-0$ & 76 \\
\hline 55 & 50.83 & 1.04 & Olean-12-ene & $000471-68-1$ & 72 \\
\hline 55 & 50.83 & 1.04 & Pyrrolo[2,3-b]indole, 1,2,3,3a,8,8 & $046479-70-3$ & 72 \\
\hline 16 & 18.15 & 1.04 & Bicyclo[5.2.0]nonane, 2-methylene- & $1000159-38-9$ & 93 \\
\hline
\end{tabular}

Table 6: Summary of GC-MS results from hexane extract analysis 
14 compounds met these criteria (probability value of at least $50 \%$ and a peak area of at least $1 \%$ ) in the results for the ethyl acetate extract analysis (table 7). In this case however, only four represented compounds with peak areas of at least $5 \%$. The first two, with a retention time of 34.27 minutes, peak area of $43.13 \%$, and probable identification values of at least $90 \%$, again represent the sesquiterpene lactone known as "achillin" or "azuleno." The third compound, with a retention time of 49.73 minutes, peak area of $7.93 \%$, and a probable identification value of $89 \%$, was identified as a tricyclic compound commonly referred to as "jaceidin." The fourth compound, with a retention time of 31.41 minutes and a peak area of $7.14 \%$, was unidentified. Further analysis with the NIST 2008 data library failed to identify this peak with a probable identification value over $50 \%$.

\begin{tabular}{|c|c|c|c|c|c|}
\hline \multicolumn{7}{|c|}{ Ethyl Acetate Extract GC-MS Results } \\
$\begin{array}{c}\text { Peak } \\
\text { Number }\end{array}$ & $\begin{array}{c}\text { Retention } \\
\text { Time }\end{array}$ & Area & Library/ID & CAS\# & $\begin{array}{c}\text { Probable } \\
\text { Identification }\end{array}$ \\
\hline 70 & 34.27 & 43.13 & Azuleno[4,5-b]furan-2,7-dione, 3,3 & $005956-04-7$ & 91 \\
\hline 70 & 34.27 & 43.13 & Achillin & $071616-00-7$ & 90 \\
\hline 89 & 49.73 & 7.93 & 4 H-1-Benzopyran-4-one, 5,7-dihydro & $010173-01-0$ & 89 \\
\hline 62 & 31.41 & 7.14 & Unidentified & NA & NA \\
\hline 6 & 10.95 & 4.36 & Bicyclo[2.2.1]heptan-2-one, 1,7,7- & $000464-49-3$ & 95 \\
\hline 6 & 10.95 & 4.36 & Camphor & $000076-22-2$ & 94 \\
\hline 6 & 10.95 & 4.36 & Bicyclo[2.2.1]heptan-2-one, 1,7,7- & $000464-48-2$ & 97 \\
\hline 14 & 13.49 & 3.29 & 1H-Indene, octahydro-5-methyl- & $019744-64-0$ & 50 \\
\hline 73 & 35.58 & 2.24 & Unidentified & NA & NA \\
\hline 72 & 34.82 & 1.57 & Azuleno[4,5-b]furan-2,7-dione, 3,3 & $005956-04-7$ & 96 \\
\hline 72 & 34.82 & 1.57 & Achillin & $071616-00-7$ & 98 \\
\hline 74 & 35.89 & 1.40 & Unidentified & NA & NA \\
\hline 60 & 30.76 & 1.27 & Unidentified & NA & NA \\
\hline 35 & 19.04 & 1.07 & Unidentified & NA & NA \\
\hline
\end{tabular}

Table 7: Summary of GC-MS results from ethyl acetate extract analysis 
33 compounds in the results for the water extract analysis had probable identification values of at least $50 \%$ and peak areas of at least $1 \%$ (table 8). However, only two had peak areas of greater than $5 \%$. The first, with a retention time of 34.02 minutes, a peak area of $15.51 \%$, and a probable identification value of $94 \%$, was once again the sesquiterpene lactone "azuleno." The second, with a retention time of 23.54 minutes and a peak area of $6.08 \%$, was unidentified. Further analysis with the NIST 2008 data library failed to identify this peak with probable identification value over $50 \%$. 


\begin{tabular}{|c|c|c|c|c|c|}
\hline \multicolumn{6}{|c|}{ Water Extract GC-MS Results } \\
\hline Peak \# & $\begin{array}{l}\text { Retention } \\
\text { Time }\end{array}$ & Area & Library/ID & CAS\# & $\begin{array}{c}\text { Probable } \\
\text { Identification }\end{array}$ \\
\hline 67 & 34.02 & 15.51 & Azuleno[4,5-b]furan-2,7-dione, 3,3 & 005956-04-7 & 94 \\
\hline 45 & 23.54 & 6.08 & Unidentified & NA & NA \\
\hline 65 & 32.85 & 4.24 & Neo-Inositol & $000488-54-0$ & 50 \\
\hline 64 & 32.58 & 4.00 & Allo-Inositol & 000643-10-7 & 64 \\
\hline 64 & 32.58 & 4.00 & Neo-Inositol & 000488-54-0 & 72 \\
\hline 61 & 31.34 & 3.56 & Unidentified & NA & NA \\
\hline 78 & 38.57 & 3.11 & Unidentified & NA & NA \\
\hline 86 & 46.59 & 2.95 & Unidentified & NA & NA \\
\hline 33 & 18.77 & 2.94 & Unidentified & NA & NA \\
\hline 38 & 20.43 & 2.60 & Unidentified & NA & NA \\
\hline 28 & 17.22 & 2.34 & Unidentified & NA & NA \\
\hline 15 & 13.49 & 2.01 & 2,2-Dimethyl-1-(2-methylene-cycloh & $1000187-17-4$ & 59 \\
\hline 15 & 13.49 & 2.01 & 2,5,5-Trimethyl-cyclohex-3-enone & $1000193-78-3$ & 53 \\
\hline 15 & 13.49 & 2.01 & 1,3-Pentadiene, 2,4-dimethyl- & $001000-86-8$ & 50 \\
\hline 46 & 23.82 & 1.89 & Unidentified & NA & NA \\
\hline 82 & 40.32 & 1.76 & Thieno[2,3-b]thiophene, 2-ethyl- & 005912-01-6 & 52 \\
\hline 29 & 17.35 & 1.68 & Unidentified & NA & NA \\
\hline 52 & 26.21 & 1.67 & Unidentified & NA & NA \\
\hline 35 & 18.99 & 1.64 & 3,6-Octadien-1-ol, 3,7-dimethyl-, & 005944-20-7 & 83 \\
\hline 72 & 35.46 & 1.62 & Unidentified & NA & NA \\
\hline 54 & 28.71 & 1.37 & Unidentified & NA & NA \\
\hline 63 & 31.94 & 1.37 & Allo-Inositol & 000643-10-7 & 50 \\
\hline 63 & 31.94 & 1.37 & Scyllo-Inositol & $000488-59-5$ & 50 \\
\hline 63 & 31.94 & 1.37 & Inositol & 006917-35-7 & 50 \\
\hline 79 & 39.20 & 1.21 & Unidentified & NA & NA \\
\hline 73 & 35.80 & 1.20 & Unidentified & NA & NA \\
\hline 75 & 37.65 & 1.12 & Unidentified & NA & NA \\
\hline 87 & 49.63 & 1.08 & 4H-1-Benzopyran-4-one, 5,7-dihydro & 010173-01-0 & 83 \\
\hline 49 & 24.68 & 1.03 & Phenol, 2,6-dimethoxy-4-(2-propeny & $006627-88-9$ & 91 \\
\hline 49 & 24.68 & 1.03 & 2-Propenoic acid, 3-(4-hydroxy-3-m & $001135-24-6$ & 89 \\
\hline 51 & 25.52 & 1.03 & Phenol, 4-(3-hydroxy-1-propenyl)-2 & $000458-35-5$ & 89 \\
\hline 51 & 25.52 & 1.03 & $(+)-s-2-P h e n e t h a n a m i n e, 1-m e t h y l-N$ & $1000127-89-6$ & 50 \\
\hline 51 & 25.52 & 1.03 & 2-Butanone, 3-(phenylthio)- & $013023-53-5$ & 64 \\
\hline
\end{tabular}

Table 8: Summary of GC-MS results from water extract analysis 


\section{Column Chromatography}

Following analysis of the GC-MS results, newly created hexane extracts were subjected to solid-phase extraction to potentially identify the compound(s) responsible for growth inhibition of Staphylococcus aureus. Two hexane extracts were created by direct treatment of the plant material with this solvent: The first extract was created by the boiling of $5.0067 \mathrm{~g}$ of Artemisia tridentata plant material, dried and ground using a mortar and pestle, in $50 \mathrm{ml}$ hexane for approximately 45 minutes using a reflux condenser. After treatment, the supernatant was decanted and filtered through cotton. 50 $\mathrm{ml}$ fresh hexane was added to the plant material, and the process repeated. The pooled filtrates were subjected to an additional filtration using Whatman \#1 paper to remove still visible particulate matter. The second extract was created by adding $5.0076 \mathrm{~g}$ more of dried and ground plant material to the original material, and repeating a similar process. Solvent was removed from both samples using a rotary evaporator and desiccation under vacuum. Each resulting extract was suspended in $5 \mathrm{ml}$ DMSO, filtered first through a 0.2 $\mu \mathrm{m}$ syringe filter, and then through Whatman \#1 filter paper to remove still visible particulate matter.

Column chromatography of the extracts was based on the method of Mojarrab et al. $^{113} .3 \mathrm{ml}$ of a solution composed of $95 \%$ methanol and 5\% DMSO was passed through a Sep-Pak C18 cartridge (Waters) to pre-treat the column. This process was repeated four times. The first hexane extract was then passed through the column, after which the column was washed using the methanol/DMSO solution in four applications of $3 \mathrm{ml}$ each. The column was then treated successively with $3 \mathrm{ml}$ volumes of acetonitrile/water solutions to create fractioned samples of the hexane extract. The first solution contained 
$10 \%$ acetonitrile and $90 \%$ water, with each following solution containing a $10 \%$ incremental increase in acetonitrile, thus the last solution consisted only of this solvent. The flow-through from each of these ten solutions was collected in separate vials. The process was then repeated with a new column and the second hexane extract, and the flow-through from each acetonitrile/water solution treatment combined with the corresponding collected flow-through from the first extract.

Solvent was removed from the column fractions using rotary evaporation. Based on the results of a previous experiment (data not shown), the $10 \%$ acetonitrile and $100 \%$ acetonitrile fractions were subjected to GC-MS analysis. $10 \mu \mathrm{l}$ of the $10 \%$ acetonitrile sample, which had an oily appearance, was re-suspended in $100 \mu 1$ of a $50 \%$ dichloromethane/methanol solution. Alternatively, as the $100 \%$ acetonitrile fraction appeared as a non-motile residue in only a trace amount, $300 \mu \mathrm{l}$ of dichloromethane/methanol solution was used to wash the vial containing this sample. 5 $\mu 1$ of each resulting solution was injected (split mode, 1:10 ratio) into an Agilent 6890 Gas Chromatograph equipped with an Agilent 5973 single quadrupole mass spectrometer. The $10 \%$ acetonitrile fraction sample yielded 14 peaks (figure 99), while the $100 \%$ acetonitrile sample yielded 34 (figure 100). Compounds were identified using the NIST 1998 data library. 


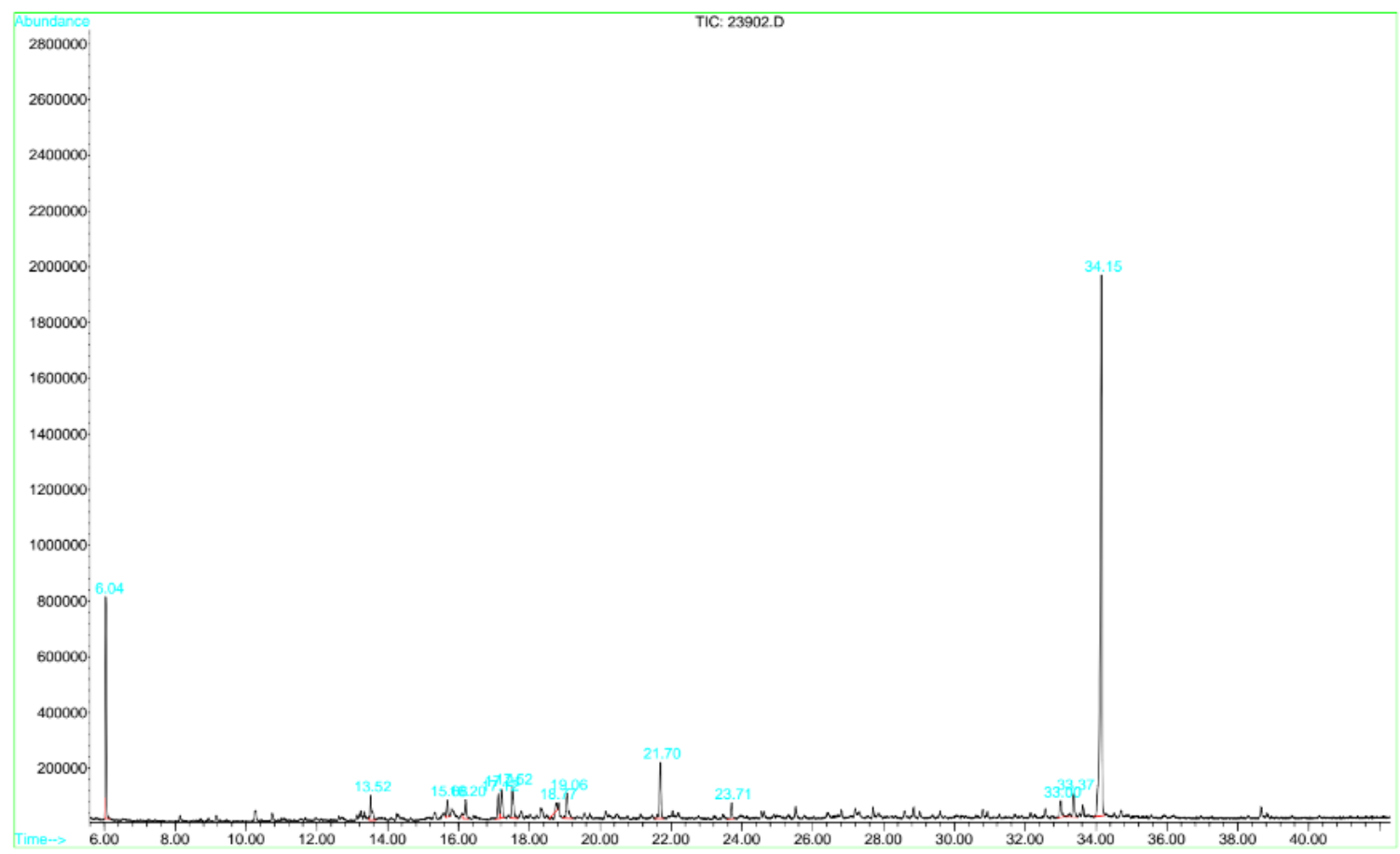

Figure 99: GC-MS results for $10 \%$ acetonitrile column fraction of hexane extract

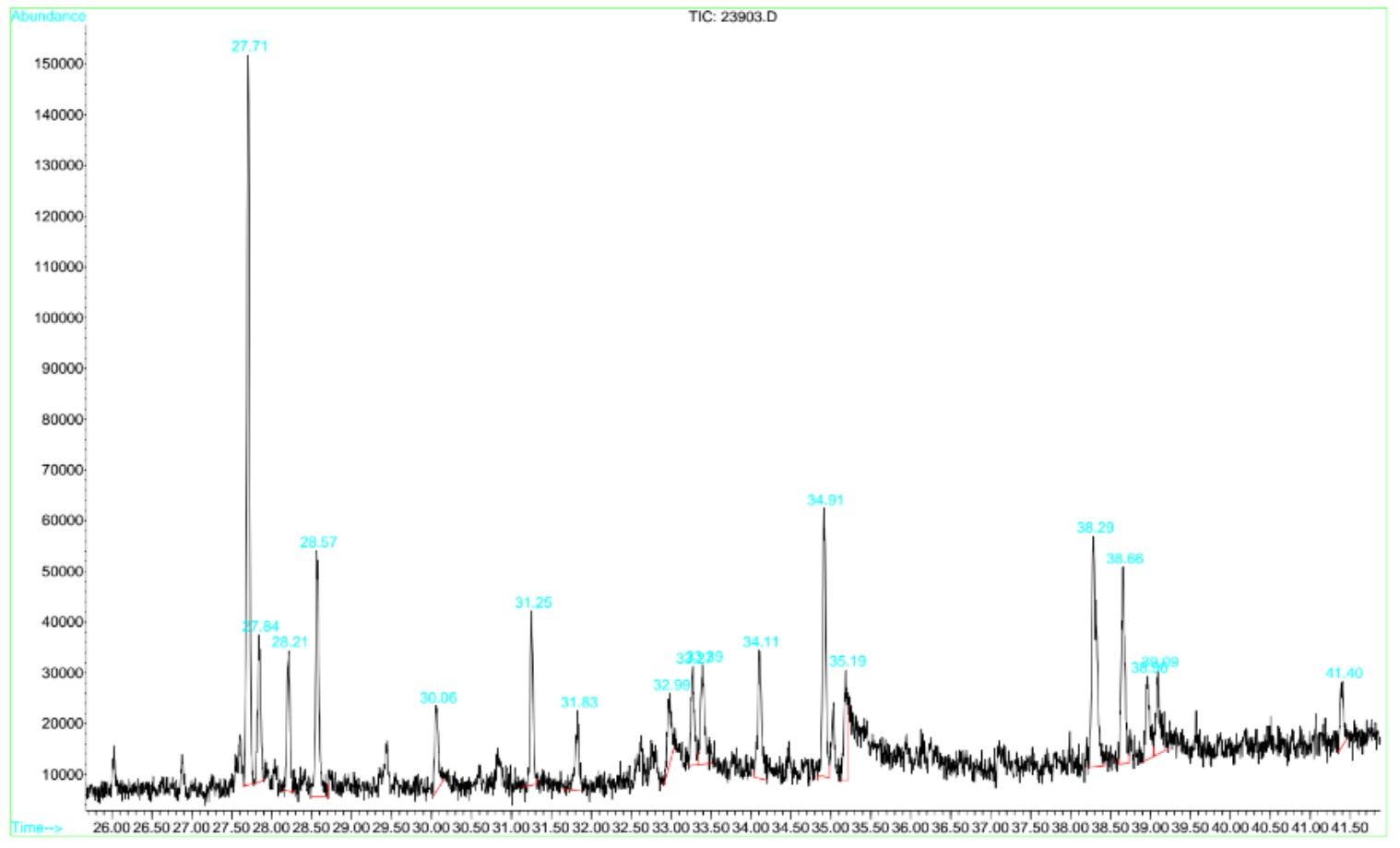

Figure 100: GC-MS results for $100 \%$ acetonitrile column fraction of hexane extract 
After rotary evaporator removal of the dichlormethane/methanol solvent from the $100 \%$ acetonitrile fraction, all ten pooled column fractionation samples were assayed for antibacterial effects versus Staphylococcus aureus. As most of the samples yielded only small amounts of visible residue after acetonitrile/water removal, each vial was washed with $500 \mu \mathrm{LB}$, and this sample-containing media added in $100 \mu \mathrm{l}$ aliquots in triplicate to the wells of a 96-well plate. An overnight culture of Staphylococcus aureus was incubated at approximately $37^{\circ} \mathrm{C}$ and $225 \mathrm{rpm}$ for about 16 hours, and diluted to an $\mathrm{OD}_{600}$ reading of 1.020 before the addition of $2 \mu \mathrm{l}$ culture to the appropriate wells. Controls (100 $\mu \mathrm{l}$ each) included untreated LB (sterility), tetracycline in LB at $25 \mu \mathrm{g} / \mathrm{ml}$ (positive), and untreated Staphylococcus aureus (negative), with each performed in triplicate. The plate was incubated at $37^{\circ} \mathrm{C}$ and $125 \mathrm{rpm}$, with measurements taken immediately prior to incubation, and at 1-hour intervals for 6 hours. As can be seen in figure 101, only the $10 \%$ acetonitrile fraction appeared to inhibit bacterial growth over this period.

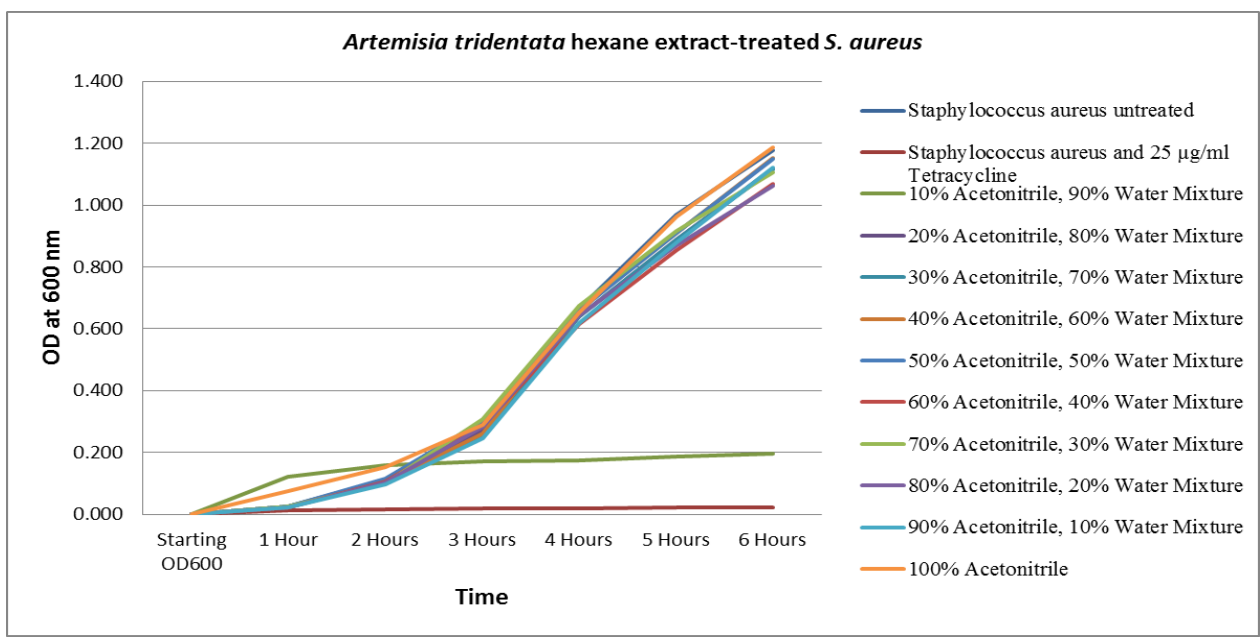

Figure 101: Antibacterial assay of hexane extract column fractions 
Compounds identified in the $10 \%$ acetonitrile fraction with a probability value of at least $50 \%$ and a peak area of at least $1 \%$, were tabulated. Seven compounds met these criteria (table 9). Of these, only the sesquiterpene lactone "achillin" or "azuleno," had a probable identification value of greater than $90 \%$, and a peak of greater than $5 \%$.

\begin{tabular}{|c|c|c|c|c|c|}
\hline \multicolumn{7}{|c|}{ Hexane Extract GC-MS Results } \\
$\begin{array}{c}\text { Peak } \\
\text { Number }\end{array}$ & $\begin{array}{c}\text { Retention } \\
\text { Time }\end{array}$ & Area & Library/ID & CAS\# & $\begin{array}{c}\text { Probable } \\
\text { Identification }\end{array}$ \\
\hline 2 & 13.52 & 2.56 & 1,3-Pentadiene, 2,4-dimethyl- & $001000-86-8$ & 59 \\
\hline 2 & 13.52 & 2.56 & $2,5,5-$ Trimethyl-cyclohex-3-enone & $1000193-78-3$ & 53 \\
\hline 11 & 23.71 & 1.31 & Methyl jasmonate & $001211-29-6$ & 93 \\
\hline 11 & 23.71 & 1.31 & Cyclopentaneacetic acid, 3-oxo-2-( & $042536-97-0$ & 93 \\
\hline 11 & 23.71 & 1.31 & (3-Oxo-2-pent-2-enylcyclopentyl)ac & $1000211-13-7$ & 95 \\
\hline 14 & 34.15 & 56.36 & Achillin & $071616-00-7$ & 99 \\
\hline 14 & 34.15 & 56.36 & Azuleno[4,5-b]furan-2,7-dione, 3,3 & $005956-04-7$ & 96 \\
\hline
\end{tabular}

Table 9: Summary of GC-MS results from $10 \%$ acetonitrile column fraction analysis 


\section{DISCUSSION}

\section{Evaluation of Results}

The results of the antibacterial assays using the ethyl acetate, hexane and water extracts derived from Artemisia tridentata plant material strongly indicate the presence of an antibacterial compound(s) capable of selective growth inhibition of Staphylococcus aureus. Given the initial bacterial inoculum levels and experimental conditions used in these assays, all three extracts significantly negatively impacted the growth of this bacterial species over a period of 6 hours in comparison to untreated controls. The ethyl acetate extract displayed statistically significant antibacterial effects at the lowest concentration $(62.5 \mu \mathrm{g} / \mathrm{ml})$, followed by the hexane extract $(125 \mu \mathrm{g} / \mathrm{ml})$, and finally the water extract $(250 \mu \mathrm{g} / \mathrm{ml})$, which demonstrated the least amount of growth inhibition. Interestingly however, at a concentration of $500 \mu \mathrm{g} / \mathrm{ml}$, it was the hexane extract-treated growth curves which most closely resembled those of the positive control.

Further, applications of these extracts in combination with the antibiotics G418 sulfate, amikacin and ampicillin enhanced the antibacterial efficacy of all three drugs. Statistically significant improvement of growth inhibition was routinely observed when these combinatorial effects were compared to those of the antibiotics alone. At the antibiotic concentration of $1.25 \mu \mathrm{g} / \mathrm{ml}$ for example, G418 sulfate and amikacin performances were enhanced by ethyl acetate extract concentrations as low as 62.5 $\mu \mathrm{g} / \mathrm{ml}$, while ampicillin was enhanced by a hexane extract concentration as low as $125 \mu \mathrm{l}$. The failure of the ethyl acetate extract to also demonstrate enhancement of ampicillin effects at the lowest concentration among the extracts is explicable upon examination of the growth curve graphs. It can be observed that in the hexane extract/ampicillin 
combination assays that the untreated controls grew to higher levels in comparison to the ethyl acetate/ampicillin combination assays, and that the final readings for combinations of extracts with $1.25 \mu \mathrm{g} / \mathrm{ml}$ ampicillin were actually higher in the latter. Thus, some of the inhibitory effects of the hexane extract/ampicillin combinations were statistically significant due to the greater growth of the untreated control in those assays, despite growth inhibition at these combined concentrations being greater in the ethyl acetate/ampicillin assays.

Over 12 hours, the extracts continued to enhance the antibacterial efficacy of these antibiotics. Combinations of the ethyl acetate and hexane extracts, though at concentrations of $500 \mu \mathrm{g} / \mathrm{ml}$, with either G418 sulfate or amikacin, strongly inhibited Staphylococcus aureus, with growth curves mimicking those of the positive control. Combined effects with ampicillin were less dramatic, which is consistent with the results of the 6-hour assays. However, the hexane extract clearly demonstrated the greatest combined effects with ampicillin in the 12-hour assays.

The assays assessing the performance of the extracts in combination did not reveal any significant enhancement of antibacterial efficacy which might suggest the presence of additive or synergistic compounds. However, the values for the hexane/ethyl acetate extract combinations showed enough reduction of growth inhibition to suggest possible interference or antagonism between the two. Yet it is also possible that these elevated growth levels were due to nutrient enrichment of the media by the extracts, which resulted in more rapid bacterial growth. Though not discussed in the reviewed literature, it is important to consider that along with potential antibacterial compounds, chemical extraction of plant material also potentially withdraws many chemicals which 
can be used by bacteria as energy sources, thereby enriching the level and variety of nutrients in media when added in antibacterial assays. In many instances in this study, lower concentrations of extracts alone or in combination with antibiotics were observed to exhibit growth curves surpassing those of the untreated controls. This can complicate the assessment of an extract's antibacterial efficacy, as its true potency may be diminished by the promotion of bacterial growth due to these additional compounds. In any case, as there was still significant growth inhibition by the hexane/ethyl acetate extract combination in comparison to the untreated control at the highest concentrations tested, antagonist effects could be considered moderate.

The antibacterial efficacy of each extract was lessened when tested against the ampicillin resistant strain BAA-44, with significant growth inhibition seen only at the highest tested concentration $(500 \mu \mathrm{g} / \mathrm{ml})$ of the ethyl acetate and hexane extracts. As ampicillin resistance in this strain is the result of the action of a $\beta$-lactamase, this lessened efficacy is difficult to explain. If this enzyme were to react in some manner with the antibacterial compound(s) in the extracts, a similar lessening of the combined efficacy of the extracts with ampicillin would be expected. However, both the ethyl acetate and hexane extracts significantly improved the growth inhibition of all ampicillin concentrations tested in comparison to their performances alone, with the growth curves of hexane extract/ampicillin combinations mimicking those of the positive control. It is possible that the antibacterial efficacy of the extracts, when applied alone, was masked by the slow growth of the untreated controls in these assays.

Under the experimental conditions tested, none of the extracts displayed any inhibitory effects on Staphylococcus aureus biofilm formation. The 10-hour incubation 
period allowed for biofilm formation in these assays exceeded the antibacterial assays by 4 hours, so this may have diminished extract effects to some extent. Still, the quantification of crystal violet binding indicated higher biofilm formation in the extracttreated samples. It is again possible that the extracts enriched the test media, and that this resulted in more rapid bacterial growth after the antibacterial capacity of the extracts was overwhelmed. Alternatively, as the plates were incubated without rotation, biofilm formation early in the assay may have protected the cells within from the extracts, rendering them ineffective.

The extracts, alone or in combination with antibiotics, also had no effects in the treatment of static biofilms. The length of sample treatment in these assays was 12 hours. Given the results of extract/antibiotic combinations from the earlier 12-hour antibacterial assays, and the use of $1 \mathrm{mg}$ concentrations of antibiotics in the treatment of the static biofilms, some reduction of the biofilms might have been expected. The lack of positive results is most likely attributable to the resistive capabilities of the biofilm to the extracts and antibiotics. However, if the antibacterial effects of the extracts are bacteriostatic as opposed to bactericidal in nature, this could also explain their ineffectiveness versus a static biofilm.

In the serial dilution and plating of extract-treated Staphylococcus aureus samples, the overnight cultures, after adjustment to an $\mathrm{OD}_{600}$ reading approximating 1.000 , yielded a colony count average of $1.45 \times 10^{8}$ cells $/ \mathrm{ml}$. Given the approximate $2 \mu \mathrm{l}$ culture: $100 \mu \mathrm{l}$ media ratio that was used in both these assays and the earlier antibacterial assays, this number would translate to bacterial inoculum concentrations of about $2.9 \mathrm{x}$ $10^{6}$ cells $/ \mathrm{ml}\left(\left[1.45 \times 10^{8}\right.\right.$ cell $\left.\left./ \mathrm{ml} \times 0.002\right] / 0.1 \mathrm{ml}\right)$, considerably less than the $1.52-1.68 \mathrm{x}$ 
$10^{7}$ cells $/ \mathrm{ml}$ estimated for the earlier antibacterial assays using the standard Escherichia coli value for an $\mathrm{OD}_{600}$ reading of 1.000 . However, these experimental numbers are estimates, based on only three trials, and neglecting to take into consideration the optical alignment of the spectrophotometer ${ }^{114}$.

The average colony count of $3.79 \times 10^{6}$ cells $/ \mathrm{ml}$ for the untreated control sampled after 25 minutes of incubation is reasonably representative of the calculated inoculum level, given that the experiment was performed only three times. The comparatively low average colony count of $1.73 \times 10^{5}$ cells $/ \mathrm{ml}$ for the tetracycline-treated samples could either be due to a bacteriostatic effect on cells in the inoculums which were already in the process of dividing, or to a bactericidal effect of the aminoglycoside at this concentration to cell ratio ${ }^{115}$. The average colony count for the 2-hour untreated sample $\left(2.8 \times 10^{7}\right.$ cells $/ \mathrm{ml}$ ) indicates 3 to 4 rounds of division by the cells in the initial inoculum, which reinforces an estimated doubling time of around 35 minutes under these experimental conditions. Therefore, as the colony counts for the extract-treated samples closely approximated that of the untreated control after 25 minutes, these results are indicative of a bacteriostatic, as opposed to bactericidal, mechanism of action, which is in agreement with the literature.

The toxicity assay results imply that at the concentrations which demonstrated significant growth inhibition in the earlier antibacterial assays, these extracts are moderately to very lethal against MDA-MB-231 cells. They could therefore be hazardous to human health if taken internally, which would also be in agreement with the literature. However, it is possible that the antibiotic compound(s) in the extracts are not responsible for this toxicity, and that the use of an alternative solvent in the creation of an 
extract, or the physical isolation of the antibiotic compound(s), might result in safer products. Also, these results do not necessarily discount the usefulness of extracts or compounds derived from Artemisia tridentata in external applications, such as those also described in the literature.

The GC-MS results for the three extracts indicate achillin, a sesquiterpene lactone, as the most likely candidate responsible for these effects, as this was the only compound identified as present in all three tested samples under the chromatographic and mass spectral analytical conditions employed. The identification of this compound in the hexane extract column fraction exhibiting antibacterial activity further supports this conclusion. However, it should be noted that the antibacterial assay was performed by adding media directly to the samples without regard to concentration, due to the fractionation resulting in very limited individual volumes. The $10 \%$ acetonitrile sample demonstrating Staphylococcus aureus growth inhibition consisted of perhaps as much as 20-30 $\mu 1$, a volume larger than that typically added to achieve the highest concentration tested in the extract assays $(500 \mu \mathrm{g} / \mathrm{ml})$. Also, as the concentration of individual compounds was likely changed as a result of the column fractionation process, there exists the possibility, however unlikely, that another compound(s) present in this fraction was responsible for this antibacterial effect, and that this effect was only exerted due to the use of an excessive concentration of this compound(s). It should also be noted that GC-MS analysis of the three extract samples revealed additional compounds unique to each extract which may possess antibacterial properties. Therefore, it is possible that achillin is not the only antibacterial compound present in Artemisia tridentata, and that its natural bacterial defense mechanisms consist of the use of more than one compound. 
Nevertheless, the crediting of the antibacterial properties of this plant to the presence of a sesquiterpene lactone is in line with existing literature on the subject. Sesquiterpene lactones are lipophilic fifteen-carbon compounds believed to be formed through the condensation of three isoprene units, followed by cyclization and oxidative transformation to cis or trans-fused lactones ${ }^{101}$. These compounds are typically found in glandular trichomes and secretory canals of plants, and are believed to be one of the main mechanisms of plant microbial defense, disrupting the bacterial cell membrane through interaction of the constituent polar groups with the phospholipid membrane ${ }^{101}$. Indeed, it has been suggested that this class of compound is perhaps the largest of those to be found to exist as secondary metabolites in plants, with over 5,000 structures identified to date $^{101}$.

While assays investigating the potential role of $\mathrm{pH}$ or DNA-damaging effects failed to demonstrate positive results, the ethidium bromide assay and electron microscopy results both support the idea of a mechanism of action involving bacterial membrane disruption. The ethidium bromide assay results for example, demonstrated enhanced binding to cellular DNA in the presence of the extracts, which is indicative of increased membrane permeability, while the transmission electron microscopy results indicated cellular rupture in the ethyl acetate and hexane extract-treated samples. However, these results alone cannot completely discount the possibility of autocidal activity resulting from extract toxicity towards Staphylococcus aureus. Experimental Shortcomings

As noted in the introductory literature review, a completely exhaustive examination of the antibacterial properties of a plant would likely require an enormous 
amount of trial and error using a variety of sampling, extraction, testing and identification methods. Therefore, it is important to acknowledge the limitations of this particular study. First, as reported, the Artemisia tridentata plant material obtained was collected from one location during the month of November. It is possible that sample collections from other locations or from the same location at a different time(s) would have resulted in variability in the level of achillin or other compounds contributing to the antibacterial activity of the plant extracts. For example, it has been reported that autumn is the time of year when the essential oil content of sagebrush species is at its peak ${ }^{99}$. However, it has also been reported that this species remains photosynthetically active even during periods of cold, thus these fluctuations may be minimal ${ }^{116}$ if metabolic energy availability is consistent throughout the year. Second, the extracts tested were made predominantly from leaf material, as limited amounts of stems or flowers, and no roots were obtained. The chemical composition and antibacterial activity of extracts created from different parts of this plant may vary, perhaps significantly if the roots possess excretory secretory canals containing achillin or other antibacterial compounds.

Additionally, in the creation of the extracts used for the bulk of the antibacterial testing, the original solvent applied to the plant material was methanol, with subsequent extracts created by fractionation of the resulting solution. It is very likely that methanol failed to extract all constituent compounds in the treated plant material. Had time and the availability of plant material allowed, the treatment of individual plant samples with various solvents on an individual basis, or the sequential treatment of a single sample with these solvents, may have potentially resulted in extracts with greater and/or widerranging antibacterial properties. Further, the antibacterial testing included only four 
bacterial species, and only three antibiotics were screened for additive or synergistic effects in combination with the plant extracts. A more expansive screening of both may have revealed greater potential applicability of the extracts. Finally, attempts to determine the chemical composition of the extracts were potentially limited by the approaches taken in the chromatographic and spectral analyses. For example, it is possible that the chromatographic settings used, the molecular mass cutoff values of the mass spectral analyses, or the use of outdated reference databases resulted in the misidentification or failed identification of a relevant compound(s) in the samples. Future Experiments

We were unable to obtain purified achillin through commercial sources. The physical isolation and purification of achillin from Artemisia tridentata plant material would allow for a more accurate assessment of its antibacterial potency and mechanism of action against Staphylococcus aureus, as well as perhaps some other bacterial species with similar cell membrane compositions. Such testing would also reveal whether these effects, exhibited by the chemical extracts in the experiments described herein, are the result of this compound alone, or if it is possible that one or more of the other chemicals identified by GC-MS analysis as present in these extracts contributed to bacterial growth inhibition in some manner. Further, the chemical structure of achillin suggests 16 possible stereo-configurations. While the bioactivity of sesquiterpene lactones has been reported as attributable to the presence of the $\alpha$-methylene- $\gamma$-lactone group ${ }^{101}$, isolation and purification would allow for structural determination of the specific stereoisomer produced by this plant species, and perhaps allow for the design of experiments to test whether this configuration is relevant to this compound's antibacterial activity. 
It would also be interesting to modify and repeat the antibacterial assays testing the methanol extracts of the fifteen plant species screened prior to Artemisia tridentata. The demonstration of any "meaningful" antibacterial activity possessed by the extracts created from this plant was only observed after reducing the bacterial inoculum level in the assays performed. Further, these effects were initially detected using a working concentration as high as $1000 \mu \mathrm{g} / \mathrm{ml}$. This illustrates the difficulties in detecting antibacterial properties in plant investigations, as these properties would likely have been missed without these experimental modifications. In an antibacterial assay, the working concentration of the tested chemical and the inoculum level are interdependent, with either value relatively meaningless without consideration of the other. Therefore, a better experimental approach in the future, for example, may be to initially test extracts using a pre-determined, set value for working concentration, doing so against a gradient of bacterial inoculum levels. Such an assay design might allow for a faster and more practical assessment of an extract's potency.

Finally, with regards to further experiments involving Artemisia tridentata, as well as other plants, it would be beneficial to expand the number of pathogenic bacterial species assayed, as the antibacterial effects of some plants may be exerted against a very limited number of bacteria, and including a greater number of test species may help detect them. Also, as described in the literature review, comparisons of results from testing bacterial species with different phenotypes can be useful in discerning antibacterial mechanisms of action. Similarly, when investigating combined applications with antibiotics, a greater number of drugs representing different classes should be 
included, which will improve the odds of discovering additive or synergistic effects between these and the plant extracts. 


\section{REFERENCES}

1. Eldeen IM, Elgorashi EE, van Staden J. Antibacterial, anti-inflammatory, anticholinesterase and mutagenic effects of extracts obtained from some trees used in South African traditional medicine. J Ethnopharmacol. 2005;102(3):457-464.

2. Tekwu EM, Pieme AC, Beng VP. Investigations of antimicrobial activity of some Cameroonian medicinal plant extracts against bacteria and yeast with gastrointestinal relevance. J Ethnopharmacol. 2012;142(1):265-273.

3. Ugoh SC, Agarry OO, Garba SA. Studies on the antibacterial activity of Khaya senegalensis [(Desr.) A. Juss)] stem bark extract on Salmonella enterica subsp. enterica serovar Typhi [(ex Kauffmann and Edwards) Le Minor and Popoff. Asian Pac J Trop Biomed. 2014;4(Suppl 1):S279-83.

4. Kaneria M, Baravalia Y, Vaghasiya Y, Chanda S. Determination of antibacterial and antioxidant potential of some medicinal plants from saurashtra region, India. Indian J Pharm Sci. 2009;71(4):406-412.

5. Tchouya GR, Souza A, Tchouankeu JC, et al. Ethnopharmacological surveys and pharmacological studies of plants used in traditional medicine in the treatment of HIV/AIDS opportunistic diseases in Gabon. J Ethnopharmacol. 2015;162:306-316.

6. Adwan G, Abu-Shanab B, Adwan K. Antibacterial activities of some plant extracts alone and in combination with different antimicrobials against multidrug-resistant Pseudomonas aeruginosa strains. Asian Pacific journal of tropical medicine. 2010;3(4):266-269.

7. Dusan M, Ruzica A, Jasna I, Irena Z. Investigation of antibacterial activity of supercritical extracts of plants, as well as of extracts obtained by other technological processes on some bacteria isolated from animals. Acta Vet (Beograd). 2009;59(5-6):557568.

8. Oskay M, Oskay D, Kalyoncu F. Activity of some plant extracts against multi-drug resistant human pathogens. Iranian journal of pharmaceutical research : IJPR. 2009;8(4):293-300.

9. Nuno G, Zampini IC, Ordonez RM, Alberto MR, Arias ME, Isla MI. Antioxidant/antibacterial activities of a topical phytopharmaceutical formulation containing a standardized extract of Baccharis incarum, an extremophile plant species from Argentine Puna. Phytother Res. 2012;26(11):1759-1767.

10. Aqil F, Ahmad I, Owais M. Evaluation of anti-methicillin-resistant Staphylococcus aureus (MRSA) activity and synergy of some bioactive plant extracts. Biotechnol J. 2006;1(10):1093-1102. 
11. Ohene-Agyei T, Mowla R, Rahman T, Venter H. Phytochemicals increase the antibacterial activity of antibiotics by acting on a drug efflux pump. Microbiologyopen. 2014;3(6):885-896.

12. Reverter M, Bontemps N, Lecchini D, Banaigs B, Sasal P. Use of plant extracts in fish aquaculture as an alternative to chemotherapy: Current status and future perspectives. Aquaculture. 2014;433:50-61.

13. Miceli A, Aleo A, Corona O, Sardina MT, Mammina C, Settanni L. Antibacterial activity of Borago officinalis and Brassica juncea aqueous extracts evaluated in vitro and in situ using different food model systems. Food control. 2014;40:157-164.

14. Korpe DA, Iseri OD, Sahin FI, Cabi E, Haberal M. High-antibacterial activity of Urtica spp. seed extracts on food and plant pathogenic bacteria. Int J Food Sci Nutr. 2013;64(3):355-362.

15. Tomlinson S, Palombo EA. Characterisation of antibacterial Australian medicinal plant extracts by investigation of the mechanism of action and the effect of interfering substances. J Basic Microbiol. 2005;45(5):363-370.

16. Basim E, Basim H, Özcan M. Antibacterial activities of Turkish pollen and propolis extracts against plant bacterial pathogens. J Food Eng. 2006;77(4):992-996.

17. Kotan R, Dadasoglu F, Karagoz K, et al. Antibacterial activity of the essential oil and extracts of Satureja hortensis against plant pathogenic bacteria and their potential use as seed disinfectants. Scientia horticulturae. 2013;153:34-41.

18. Abdollahzadeh E, Rezaei M, Hosseini H. Antibacterial activity of plant essential oils and extracts: The role of thyme essential oil, nisin, and their combination to control Listeria monocytogenes inoculated in minced fish meat. Food control. 2014;35(1):177183.

19. Khan H, Ali Khan M, Mahmood T, Choudhary MI. Antimicrobial activities of Gloriosa superba Linn (Colchicaceae) extracts. Journal of enzyme inhibition and medicinal chemistry. 2008;23(6):855-859.

20. Rocha-Gracia RdC, Arroyo MMH, Zarain PL, et al. Antibacterial activity of crude extracts from Mexican plants against methicillin-resistant Staphylococcus. African journal of biotechnology. 2011;10(61):13202-13218.

21. Razmavar S, Abdulla MA, Ismail SB, Hassandarvish P. Antibacterial activity of leaf extracts of Baeckea frutescens against methicillin-resistant Staphylococcus aureus. BioMed Research International. 2014;1-5. 
22. Pinho E, Ferreira IC, Barros L, Carvalho AM, Soares G, Henriques M. Antibacterial potential of northeastern Portugal wild plant extracts and respective phenolic compounds. BioMed Research International. 2014:1-8.

23. Karakas FP, Yildirim A, Turker A. Biological screening of various medicinal plant extracts for antibacterial and antitumor activities. Turkish journal of biology. 2012;36(6):641-652.

24. Srinivas P, Reddy SR. Screening for antibacterial principle and activity of Pedalium murex Linn. Proceedings of the National Academy of Sciences, India.Section B: Biological sciences. 2014;84(4):1083-1090.

25. Soberon JR, Sgariglia MA, Sampietro DA, Quiroga EN, Vattuone MA. Antibacterial activity of plant extracts from northwestern Argentina. J Appl Microbiol. 2007;102(6):1450-1461.

26. Yasunaka K, Abe F, Nagayama A, et al. Antibacterial activity of crude extracts from Mexican medicinal plants and purified coumarins and xanthones. J Ethnopharmacol. 2005;97(2):293-299.

27. Alabri THA, Al Musalami AHS, Hossain MA, Weli AM, Al-Riyami Q. Comparative study of phytochemical screening, antioxidant and antimicrobial capacities of fresh and dry leaves crude plant extracts of Datura metel L. Journal of King Saud University.Science. 2014;26(3):237-243.

28. Yildirim AB, Karakas FP, Turker AU. In vitro antibacterial and antitumor activities of some medicinal plant extracts, growing in Turkey. Asian Pac J Trop Med. 2013;6(8):616-624.

29. Szabóová R, Laukova A, Chrastinova L, et al. Benefficial effect of plant extracts in rabbit husbandry. Acta veterinaria Brno. 2012;81(3):245-250.

30. Stagos D, Portesis N, Spanou C, et al. Correlation of total polyphenolic content with antioxidant and antibacterial activity of 24 extracts from Greek domestic Lamiaceae species. Food Chem Toxicol. 2012;50(11):4115-4124.

31. Kuppusamy P, Yusoff MM, Parine NR, Govindan N. Evaluation of in-vitro antioxidant and antibacterial properties of Commelina nudiflora $\mathrm{L}$. extracts prepared by different polar solvents. Saudi J Biol Sci. 2015;22(3):293-301.

32. Turker H, Yildirim AB, Karakas FP, Koyluoglu H. Antibacterial activities of extracts from some Turkish endemic plants on common fish pathogens. Turkish journal of biology. 2009;33(1):73-78. 
33. Klančnik A, Piskernik S, Smole Mozina S, Gasperlin L, Jersek B. Investigation of some factors affecting the antibacterial activity of rosemary extracts in food models by a food microdilution method factors affecting MIC of plant extract. Int J Food Sci Tech. 2011;46(2):413-420.

34. Lee JH, Cho S, Paik HD, et al. Investigation on antibacterial and antioxidant activities, phenolic and flavonoid contents of some thai edible plants as an alternative for antibiotics. Asian-Australas J Anim Sci. 2014;27(10):1461-1468.

35. Al Akeel R, Al-Sheikh Y, Mateen A, Syed R, Janardhan K, Gupta VC. Evaluation of antibacterial activity of crude protein extracts from seeds of six different medical plants against standard bacterial strains. Saudi J Biol Sci. 2014;21(2):147-151.

36. Kousha A, Ringo E. Antibacterial effect of aquatic extract of Heracleum spp. hogweed plants from Europe on thirteen different bacteria. Pharmaceutical chemistry journal. 2015;48(10):677-680.

37. Chah KF, Eze CA, Emuelosi CE, Esimone CO. Antibacterial and wound healing properties of methanolic extracts of some Nigerian medicinal plants. $J$ Ethnopharmacol. 2006;104(1-2):164-167.

38. Vermaak I, Viljoen AM, Hamman JH, Van Vuuren SF. The effect of simulated gastrointestinal conditions on the antimicrobial activity and chemical composition of indigenous South African plant extracts. S Afr J Bot. 2009;75(3):594-599.

39. Rodrigues L, Duarte A, Figueiredo AC, et al. Chemical composition and antibacterial activity of the essential oils from the medicinal plant Mentha cervina L. grown in Portugal. Medicinal chemistry research. 2012;21(11):3485-3490.

40. Smida I, Charpy-Roubaud C, Cherif SY, et al. Antibacterial properties of extracts of Ludwigia peploides subsp. montevidensis and Ludwigia grandiflora subsp. hexapetala during their cycle of development. Aquat Bot. 2015;121:39-45.

41. Mooney EH, Martin AA, Blessin RP. Effects of light environment on recovery from harvest and antibacterial properties of Osha Ligusticum porteri (Apiaceae). Econ Bot. 2015;69(1):72-82.

42. Ocheng F, Bwanga F, Joloba M, Borg-Karlson AK, Gustafsson A, Obua C. Antibacterial activities of extracts from Ugandan medicinal plants used for oral care. $J$ Ethnopharmacol. 2014;155(1):852-855.

43. Voravuthikunchai SP, Limsuwan S. Medicinal plant extracts as anti-Escherichia coli O157:H7 agents and their effects on bacterial cell aggregation. J Food Prot. 2006;69(10):2336-2341. 
44. Kenny O, Brunton NP, Walsh D, Hewage CM, McLoughlin P, Smyth TJ. Characterisation of antimicrobial extracts from dandelion root (Taraxacum officinale) using LC-SPE-NMR. Phytother Res. 2015;29(4):526-532.

45. Kothari V, Gupta A, Naraniwal M. Comparative study of various methods for extraction of antioxidant and antibacterial compounds from plant seeds. Journal of Natural Remedies. 2012;12(2):162-173.

46. Roy S, Rao K, Bhuvaneswari CH, Giri A, Mangamoori LN. Phytochemical analysis of Andrographis paniculata extract and its antimicrobial activity. World J Microbiol Biotechnol. 2010;26(1):85-91.

47. Regazzoni L, Arlandini E, Garzon D, Santagati NA, Beretta G, Maffei Facino R. A rapid profiling of gallotannins and flavonoids of the aqueous extract of Rhus coriaria $\mathrm{L}$. by flow injection analysis with high-resolution mass spectrometry assisted with database searching. J Pharm Biomed Anal. 2013;72:202-207.

48. Stanojevic D, Comic L, Stefanovic O, Solujic-Sukdolak S. In vitro synergistic antibacterial activity of Salvia officinalis 1. and some preservatives. Archives of biological sciences. 2010;62(1):167-174.

49. Ganie SA, Jan A, Muzaffar S, Zargar BA, Hamid R, Zargar MA. Radical scavenging and antibacterial activity of Arnebia benthamii methanol extract. Asian Pac J Trop Med. 2012;5(10):766-772.

50. Mahida Y, Mohan JSS. Screening of Indian plant extracts for antibacterial activity. Pharm Biol. 2006;44(8):627-631.

51. Jesionek W, Choma IM, Majer-Dziedzic B, Malinowska I. Screening bacterial and radical scavenging properties of chosen plant extracts using thin-layer chromatographydirect bioautography. J Liq Chromatogr Rel Technol. 2014;37(20):2882-2891.

52. Hochfellner C, Evangelopoulos D, Zloh M, et al. Antagonistic effects of indoloquinazoline alkaloids on antimycobacterial activity of evocarpine. J Appl Microbiol. 2015;118(4):864-872.

53. Adwan G, Mhanna M. Synergistic effects of plant extracts and antibiotics on Staphylococcus aureus strains isolated from clinical specimens. Middle-East Journal of Scientific Research. 2008;3(3):134-139.

54. Motz VA, Bowers CP, Kneubehl AR, et al. Efficacy of the saponin component of impatiens capensis Meerb.in preventing urushiol-induced contact dermatitis. $J$ Ethnopharmacol. 2015;162:163-167. 
55. Tolmacheva AA, Rogozhin EA, Deryabin DG. Antibacterial and quorum sensing regulatory activities of some traditional Eastern-European medicinal plants. Acta Pharm. 2014;64(2):173-186.

56. Chatterjee SK, Bhattacharjee I, Chandra G. In vitro synergistic effect of doxycycline \& ofloxacin in combination with ethanolic leaf extract of Vangueria spinosa against four pathogenic bacteria. Indian J Med Res. 2009;130(4):475-478.

57. Salehi P, Sonboli A, Eftekhar F, Nejad-Ebrahimi S, Yousefzadi M. Essential oil composition, antibacterial and antioxidant activity of the oil and various extracts of Ziziphora clinopodioides subsp. rigida (BOISS.) RECH. f. from Iran. Biol Pharm Bull. 2005;28(10):1892-1896.

58. Ashokkumar S, Ravi S, Kathiravan V, Velmurugan S. Synthesis of silver nanoparticles using A. indicum leaf extract and their antibacterial activity. Spectrochim Acta A Mol Biomol Spectrosc. 2015;134:34-39.

59. Ambika S, Sundrarajan M. Antibacterial behaviour of Vitex negundo extract assisted $\mathrm{ZnO}$ nanoparticles against pathogenic bacteria. J Photochem Photobiol B. 2015;146:5257.

60. Khan M, Khan ST, Khan M, et al. Antibacterial properties of silver nanoparticles synthesized using Pulicaria glutinosa plant extract as a green bioreductant. Int J Nanomedicine. 2014;9:3551-3565.

61. Klancnik A, Piskernik S, Jersek B, Mozina SS. Evaluation of diffusion and dilution methods to determine the antibacterial activity of plant extracts. J Microbiol Methods. 2010;81(2):121-126.

62. Aqil F, Khan MS, Owais M, Ahmad I. Effect of certain bioactive plant extracts on clinical isolates of beta-lactamase producing methicillin resistant Staphylococcus aureus. J Basic Microbiol. 2005;45(2):106-114.

63. Jiang ZL, Zhou Y, Ge WC, Yuan K. Phytochemical compositions of volatile oil from Blumea balsamifera and their biological activities. Pharmacogn Mag. 2014;10(39):346352.

64. Weckesser S, Engel K, Simon-Haarhaus B, Wittmer A, Pelz K, Schempp CM. Screening of plant extracts for antimicrobial activity against bacteria and yeasts with dermatological relevance. Phytomedicine. 2007;14(7-8):508-516.

65. Wojnicz D, Kucharska AZ, Sokol-Letowska A, Kicia M, Tichaczek-Goska D. Medicinal plants extracts affect virulence factors expression and biofilm formation by the uropathogenic Escherichia coli. Urol Res. 2012;40(6):683-697. 
66. Mekinic IG, Skroza D, Ljubenkov I, Simat V, Mozina SS, Katalinic V. In vitro antioxidant and antibacterial activity of Lamiaceae phenolic extracts: A correlation study. Food technology and biotechnology. 2014;52(1, sp. iss. si):119-127.

67. Joray MB, del Rollan MR, Ruiz GM, Palacios SM, Carpinella MC. Antibacterial activity of extracts from plants of central Argentina--isolation of an active principle from Achyrocline satureioides. Planta Med. 2011;77(1):95-100.

68. Leandro LF, Cardoso MJ, Silva SD, et al. Antibacterial activity of Pinus elliottii and its major compound, dehydroabietic acid, against multidrug-resistant strains. J Med Microbiol. 2014;63(Pt 12):1649-1653.

69. Bag A, Bhattacharyya SK, Pal NK, Chattopadhyay RR. Antibacterial potential of hydroalcoholic extracts of triphala components against multidrug-resistant uropathogenic bacteria--a preliminary report. Indian J Exp Biol. 2013;51(9):709-714.

70. Yamaguchi N, Satoh-Yamaguchi K, Ono M. In vitro evaluation of antibacterial, anticollagenase, and antioxidant activities of hop components (Humulus lupulus) addressing acne vulgaris. Phytomedicine. 2009;16(4):369-376.

71. Hussain T, Arshad M, Khan S, Sattar H, Qureshi MS. In vitro screening of methanol plant extracts for their antibacterial activity. Pak J Bot. 2011;43(1):531-538.

72. Over KF, Hettiarachchy N, Johnson MG, Davis B. Effect of organic acids and plant extracts on Escherichia coli O157:H7, Listeria monocytogenes, and Salmonella Typhimurium in broth culture model and chicken meat systems. J Food Sci. 2009;74(9):M515-21.

73. Owais M, Sharad KS, Shehbaz A, Saleemuddin M. Antibacterial efficacy of Withania somnifera (ashwagandha) an indigenous medicinal plant against experimental murine salmonellosis. Phytomedicine. 2005;12(3):229-235.

74. Toyang NJ, Ateh EN, Keiser J, et al. Toxicity, antimicrobial and anthelmintic activities of Vernonia guineensis Benth. (Asteraceae) crude extracts. J Ethnopharmacol. 2012;144(3):700-704.

75. Engels C, Schieber A, Ganzle MG. Sinapic acid derivatives in defatted Oriental mustard (Brassica juncea L.) seed meal extracts using UHPLC-DAD-ESI-MS $\mathrm{n}$ and identification of compounds with antibacterial activity. European food research \& technology. 2012;234(3):535-542.

76. Bashir S, Khan BM, Babar M, et al. Assessment of bioautography and spot screening of TLC of green tea (Camellia) plant extracts as antibacterial and antioxidant agents. Indian J Pharm Sci. 2014;76(4):364-370. 
77. Marquis reagent. https://en.wikipedia.org/wiki/Marquis_reagent. Updated 2017. Accessed 11/15, 2017.

78. Liao SG, Zhang LJ, Sun F, et al. Antibacterial and anti-inflammatory effects of extracts and fractions from Polygonum capitatum. J Ethnopharmacol. 2011;134(3):10061009.

79. Liebermann-burchard test. https://en.wikipedia.org/wiki/Liebermann\%E2\%80\%93Burchard_test. Updated 2014. Accessed 11/15, 2017.

80. Bobis O, Dezmirean DS, Tomos L, Chirila F, Al. Marghitas L. Influence of phytochemical profile on antibacterial activity of different medicinal plants against grampositive and gram-negative bacteria. Appl Biochem Microbiol. 2015;51(1):113-118.

81. Pirvu L, Hlevca C, Nicu I, Bubueanu C. Comparative studies on analytical, antioxidant, and antimicrobial activities of a series of vegetal extracts prepared from eight plant species growing in Romania. JPC.Journal of planar chromatography, modern TLC. 2014;27(5):346-356.

82. Anani K, Adjrah Y, Ameyapoh Y, et al. Effects of hydroethanolic extracts of Balanites aegyptiaca (L.) Delile (Balanitaceae) on some resistant pathogens bacteria isolated from wounds. J Ethnopharmacol. 2015;164:16-21.

83. Folin-ciocalteu reagent. https://en.wikipedia.org/wiki/Folin\%E2\%80\%93Ciocalteu_reagent. Updated 2017. Accessed 11/15, 2017.

84. Hori Y, Sato S, Hatai A. Antibacterial activity of plant extracts from azuki beans (Vigna angularis) in vitro. Phytother Res. 2006;20(2):162-164.

85. Nemereshina ON, Tinkov AA, Gritsenko VA, Nikonorov AA. Influence of Plantaginaceae species on E. coli K12 growth in vitro: Possible relation to phytochemical properties. Pharm Biol. 2015;53(5):715-724.

86. Packer L. Methods in enzymology. Academic Press, 1999.:506.

87. Chaweepack T, Chaweepack S, Muenthaisong B, Ruangpan L, Nagata K, Kamei K. Effect of galangal (Alpinia galanga Linn.) extract on the expression of immune-related genes and Vibrio harveyi resistance in Pacific white shrimp (Litopenaeus vannamei). Aquacult Int. 2015;23(1):385-399.

88. Lu C, Luo X, Luo R, et al. Assessment of antibacterial properties and the active ingredient of plant extracts and its effect on the performance of crucian carp (Carassius auratus gibelio var. E'erqisi, Bloch). J Sci Food Agric. 2013;93(4):902-909. 
89. Dadasoglu F, Aydin T, Kotan R, et al. Antibacterial activities of extracts and essential oils of three Origanum species against plant pathogenic bacteria and their potential use as seed disinfectants. J Plant Pathol. 2011;93(2):271-282.

90. Liu Y, Nielsen M, Staerk D, Jager AK. High-resolution bacterial growth inhibition profiling combined with HPLC-HRMS-SPE-NMR for identification of antibacterial constituents in Chinese plants used to treat snakebites. J Ethnopharmacol.

91. Kouidhi B, Al Qurashi YM, Chaieb K. Drug resistance of bacterial dental biofilm and the potential use of natural compounds as alternative for prevention and treatment.

Microb Pathog. 2015;80:39-49.

92. Kouidhi B, Al Qurashi, YM, Chaieb K. Drug resistance of bacterial dental biofilm and the potential use of natural compounds as alternative for prevention and treatment. abstract. Microb Pathog. 2015;80(1):39-49.

93. Mustafi S, Veisaga ML, López LA, Barbieri MA. A novel insight into dehydroleucodine mediated attenuation of Pseudomonas aeruginosa virulence mechanism. BioMed research international. 2015;1-11.

94. Ahmad R, Ali AM, Israf DA, Ismail NH, Shaari K, Lajis NH. Antioxidant, radicalscavenging, anti-inflammatory, cytotoxic and antibacterial activities of methanolic extracts of some Hedyotis species. Life Sci. 2005;76(17):1953-1964.

95. Sindhu T, Rajamanikandan S, Srinivasan P. In vitro antioxidant and antibacterial activities of methanol extract of Kyllinga nemoralis. Indian J Pharm Sci. 2014;76(2):170174.

96. Taguri T, Tanaka T, Kouno I. Antibacterial spectrum of plant polyphenols and extracts depending upon hydroxyphenyl structure. Biol Pharm Bull. 2006;29(11):22262235.

97. Smirnova G, Samoilova Z, Muzyka N, Oktyabrsky O. Influence of plant polyphenols and medicinal plant extracts on antibiotic susceptibility of Escherichia coli. J Appl Microbiol. 2012;113(1):192-199.

98. EREL ŞB, Reznicek G, ŞENOL SG, YAVAŞOĞLU NÜK, Konyalioğlu S, Zeybek AU. Antimicrobial and antioxidant properties of Artemisia L. species from western Anatolia. Turkish Journal of Biology. 2012;36(1):75-84.

99. Nagy JG, Tengerdy RP. Antibacterial action of essential oils of Artemisia as an ecological factor. I. Antibacterial action of the volatile oils of Artemisia tridentata and Artemisia nova on aerobic bacteria. Appl Microbiol. 1967;15(4):819-821. 
100. Abad MJ, Bedoya LM, Apaza L, Bermejo P. The Artemisia L. genus: a review of bioactive essential oils. Molecules. 2012;17(3):2542-2566.

101. Ivanescu B, Miron A, Corciova A. Sesquiterpene lactones from Artemisia genus: Biological activities and methods of analysis. J.Anal.Methods Chem.

2015;21(10.1155):2015.

102. Tilford GL. Edible and medicinal plants of the west. Mountain Press Publishing; 1997.

103. Austin DF. Healing with plants in the American and Mexican west. Econ Bot. 1997;51(3):327-327.

104. Carroll KC, Detrick B, Hobden JA, et al. Jawetz, melnick \& adelberg's medical microbiology. 27th ed. ed. New York: Mcgraw-Hill Education; 2016.

http://ezproxy.fiu.edu/login?url=http://accessmedicine.mhmedical.com/book.aspx?bookid $=1551$.

105. CDC - staphylococcus aureus pneumoniae in healthcare settings - HAI. http://www.cdc.gov/HAI/organisms/staph.html. Accessed 10/10/2016, 2016.

106. WHO | antimicrobial resistance: Global report on surveillance 2014. http://www.who.int/drugresistance/documents/surveillancereport/en/. Accessed 10/10/2016, 2016.

107. CDC - pseudomonas aeruginosa in healthcare settings - HAI.

http://www.cdc.gov/hai/organisms/pseudomonas.html. Accessed 10/10/2016, 2016.

108. Listeria (listeriosis) | listeria | CDC. http://www.cdc.gov/listeria/index.html. Accessed 10/10/2016, 2016.

109. General information on salmonella | salmonella |CDC.

http://www.cdc.gov/salmonella/general/index.html. Accessed 10/10/2016, 2016.

110. Agilent genomics : Tools - bio calculators.

http://www.genomics.agilent.com/biocalculators/calcODBacterial.jsp. Accessed 10/10/2016, 2016.

111. Jabra-Rizk MA, Meiller TF, James CE, Shirtliff ME. Effect of farnesol on staphylococcus aureus biofilm formation and antimicrobial susceptibility. Antimicrob Agents Chemother. 2006;50(4):1463-1469.

112. Shcherbakova I, Mitra S, Beer RH, Brenowitz M. Fast fenton footprinting: A laboratory-based method for the time-resolved analysis of DNA, RNA and proteins. Nucleic Acids Res. 2006;34(6):e48. 
113. Mojarrab M, Delazar A, Moghadam SB, et al. Armenin and isoarmenin-two prenylated coumarins from the aerial parts of artemisia armeniaca. Chemistry \& biodiversity. 2011;8(11):2097-2103.

114. nanodroptryPOSTER.pdf. https://tools.thermofisher.com/content/sfs/posters/nanodroptryPOSTER.pdf. Accessed 11/14/2017, 2017.

115. Aminoglycosides - infectious diseases - merck manuals professional edition. http://www.merckmanuals.com/professional/infectious-diseases/bacteria-andantibacterial-drugs/aminoglycosides. Accessed 11/14/2017, 2017.

116. Wright CW. Artemisia. London ; New York: Taylor \& Francis; 2002. 
VITA

STEVEN EICHELBAUM

Born, Allentown, Pennsylvania

B.S. Biology

University of Pittsburgh

Pittsburgh, PA

M.S. Microbiology

Thomas Jefferson University

Philadelphia, PA

2007-2008

Research Technician

Florida International University

Miami, FL

2008-2010

Senior Production Technician

Beckman Coulter

Hialeah, FL

2010

Assistant Scientist

Colgate-Palmolive

Piscataway, NJ

2011-2016

Doctoral Student

Florida International University

Miami, FL 